\title{
Tao Chen
}

\section{Advanced Voice and Data Solutions for Evolution of Cellular Network System}




\section{Tao Chen}

\section{Advanced Voice and Data Solutions for Evolution of Cellular Network System}

Esitetään Jyväskylän yliopiston informaatioteknologian tiedekunnan suostumuksella julkisesti tarkastettavaksi yliopiston Agora-rakennuksen auditoriossa 3 joulukuun 12. päivänä 2014 kello 13.

Academic dissertation to be publicly discussed, by permission of the Faculty of Information Technology of the University of Jyväskylä, in building Agora, auditorium 3, on December 12, 2014 at 13 o'clock.

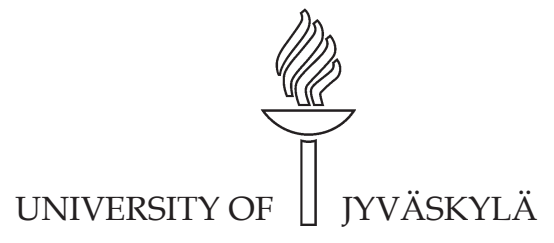

JYVÄSKYLÄ 2014 


\section{Advanced Voice and Data Solutions for Evolution of Cellular Network System}




\section{Tao Chen}

\section{Advanced Voice and Data Solutions for Evolution of Cellular Network System}

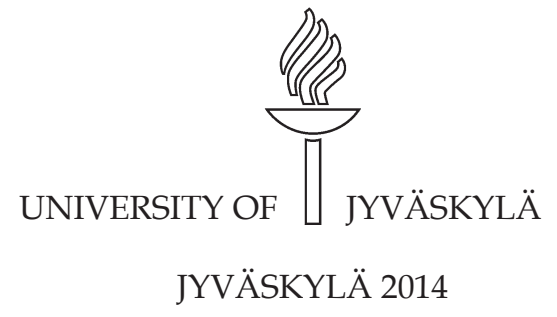


Editors

Timo Männikkö

Department of Mathematical Information Technology, University of Jyväskylä Pekka Olsbo, Ville Korkiakangas

Publishing Unit, University Library of Jyväskylä

URN:ISBN:978-951-39-5976-0

ISBN 978-951-39-5976-0 (PDF)

ISBN 978-951-39-5975-3 (nid.)

ISSN 1456-5390

Copyright (C) 2014, by University of Jyväskylä

Jyväskylä University Printing House, Jyväskylä 2014 


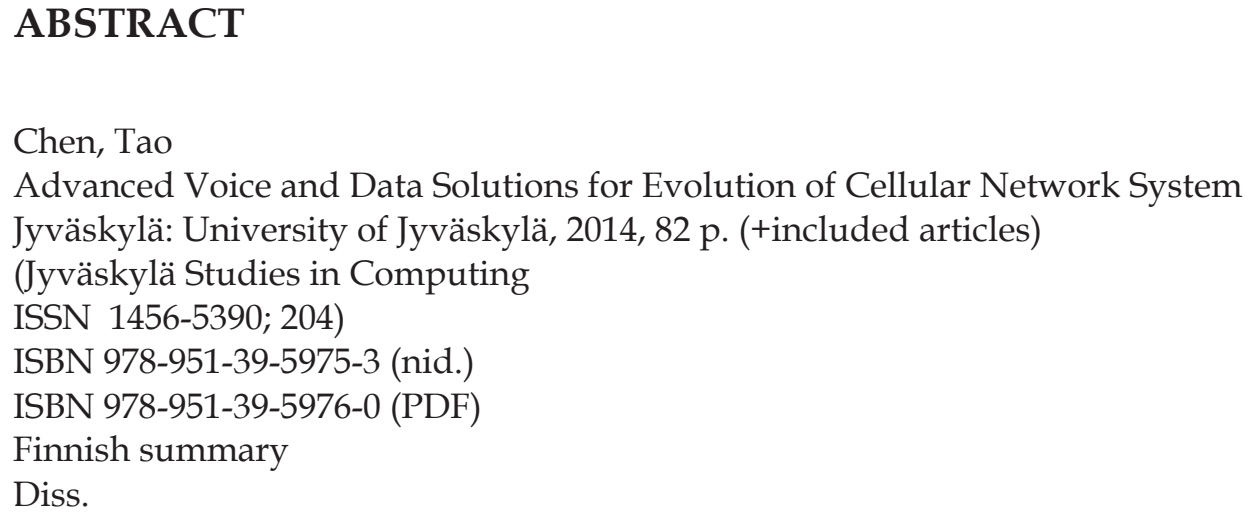

In this thesis, the solutions for efficiently offering two types of typical services (voice based RT services and data based NRT services) were investigated for evolution of a future network system.

For the voice service characterized by the low latency and the small packet size, VoIP over E-DCH associated with DPCCH gating and/or packet bundling in uplink has been investigated and proven in a compelling performance. Moreover, HS-SCCH-less operation in the downlink and the enhanced packet scheduler have been studied to further improve the user end-to-end experience. The tradeoff between reduction of CQI reports and the reliability of the link adaption has also been evaluated for consideration in performance optimization.

For the data service, a hybrid network has been integrated using deviceto-device (D2D) communication into a LTE cellular network is envisioned and investigated as one potential solution. The various RRM schemes for mode selection, power control and resource allocation are studied, which are further complemented by a time hopping mechanism and the enhanced scheduler algorithm. Additionally, asymmetric D2D operation and semi-autonomous D2D were presented as different ways for D2D implementation. D2D based cluster operation was also studied for the potential of offering multicast services. The feedback mechanism for a multicast operation was discussed for assisting the cooperative D2D operation.

In short, the VoIP based voice solutions and D2D based data solutions can be considered for future network evolution based on the studies in this thesis.

Keywords: VoIP, D2D, HSPA, LTE, RRM 
Author's address

Supervisor

Reviewers

Opponent
Tao Chen

Department of Mathematical Information Technology University of Jyväskylä

Finland

Professor Tapani Ristaniemi

Department of Mathematical Information Technology University of Jyväskylä

Finland

Professor Fu-Chun Zheng

University of Reading

UK

Dr. Jarno Niemelä

Elisa

Finland

Prof. Marcos Katz

University of Oulu

Finland 


\section{ACKNOWLEDGEMENTS}

My deepest gratitude goes first and foremost to Professor Tapani Ristaniemi, my supervisor, for helping me in the Ph.D thesis and studies with patience.

I would like to deeply thank Dr. Seppo Hämäläinen, Kari Rikkinen, Dr. Jorma Lilleberg, Sari Nielsen, Soni Koul, Dr. Yrjö Kaipainen and Dr. Kari Pehkonen for generating a good atmosphere for my research.

I would also like to show my sincere appreciation to my co-authors of the conference and journal articles and patents for their great help and professional work.

Besides, I would like to thank Timo Männikkö, Zheng Chang and MarjaLeena Rantalainen who provided huge assistance on the thesis.

Special thanks go to Wang Min for all her support, patience and understanding.

Last but not least, I would like to give my special thanks to my parents Chen Jinyan and Jin Meiling, my sister Chen Han and her husband Zhang Xin. Without their understanding and encouragement, I would not have been able to complete this work.

Jyväskylä 20.10.2014

\section{Tao CHEN}




\section{ACRONYMS}

\begin{tabular}{|c|c|}
\hline 3GPP & 3rd Generation Partnership Project \\
\hline ACK & ACKnowledgement \\
\hline ADC & Analog-to- Digital Conversion \\
\hline AGC & Automatic Gain Control \\
\hline AMR & Adaptive Multi-Rate \\
\hline AMR-NB & Adaptive Multi-Rate Narrowband \\
\hline $\mathrm{ARQ}$ & Adaptive Repeat and Request \\
\hline BS & Base Station \\
\hline CAZAC & Constant Amplitude Zero AutoCorrelation wave \\
\hline $\mathrm{CPICH}$ & Common Pilot Channel \\
\hline CQI & Channel Quality Information \\
\hline CS & Circuit Switched \\
\hline CTS & Clear to Send \\
\hline D2D & Device-to-Device \\
\hline $\mathrm{DCH}$ & Dedicated Channel \\
\hline DL & Downlink \\
\hline $\mathrm{DPCCH}$ & Dedicated Physical Control Channel \\
\hline DPDCH & Dedicated Physical Data Channel \\
\hline E-DCH & Enhanced DCH \\
\hline E-DPCCH & Enhanced DPCCH \\
\hline E-DPDCH & Enhanced DPDCH \\
\hline E-UTRAN & Enhanced UTRAN \\
\hline EVA & Extended Vehicular A \\
\hline FDD & Frequency Division Duplex \\
\hline FPC & Fractional Power Control \\
\hline GGSN & Gateway GPRS Support Node \\
\hline GTP & GPRS Tunneling Protocol \\
\hline HARQ & Hybrid Adaptive Repeat and Request \\
\hline HSDPA & High Speed Downlink Packet Access \\
\hline HS-DPCCH & High Speed DPCCH \\
\hline HS-DSCH & High Speed Downlink Shared Channel \\
\hline HSPA & High Speed Packet Access \\
\hline HS-PDSCH & High Speed Physical Downlink Shared Channel \\
\hline HS-SCCH & High Speed Shared Control Channel \\
\hline HSUPA & High Speed Uplink Packet Access \\
\hline ICI & Inter-Carrier Interference \\
\hline IEEE & Institute of Electrical and Electronics Engineers \\
\hline IMT & International Mobile Telecommunications \\
\hline IP & Internet Protocol \\
\hline ISD & Inter-site Distance \\
\hline LTE & Long Term Evolution \\
\hline MAC & Medium Access Control \\
\hline MTC & Machine Type Communications \\
\hline
\end{tabular}




\begin{tabular}{ll} 
NACK & Non-ACKnowledgement \\
NR & Noise Rise \\
NRT & Non-Real Time \\
PC & Power Control \\
PDCP & Packet Data Convergence Protocol \\
PF & Proportional Fair \\
PS & Packet Switched \\
QoS & Quality of Service \\
RLC & Radio Link Control \\
RNC & Radio Network Controller \\
ROHC & Robust Header Compression \\
RRM & Radio Resource Management \\
RT & Real-Time \\
RTP & Real-time Transport Protocol \\
RTS & Request To Send \\
RTT & Round Trip Time \\
SCS & Scheduling Candidate Set \\
SF & Spreading Factor \\
SFN & Single Frequency Network \\
SGSN & Serving GPRS Support Node \\
SID & Silence Insertion Descriptor \\
SINR & Signal to Interference and Noise Ratio \\
SRNC & Serving Radio Network Controller \\
TDD & Time Division Duplex \\
TH & Time Hopping \\
TPC & Transmit Power Control \\
TTI & Transmission Time Interval \\
UDP & Unit Data Protocol \\
UE & User Equipment \\
UL & Uplink \\
UTRAN & Universal Terrestrial Radio Access Network \\
VGA & Variable Gain Amplifier \\
VoIP & Voice over IP \\
WB & Wideband \\
WCDMA & Wideband Code Division Multiple Access \\
WLAN & Wireless Local Area Network \\
& \\
\hline
\end{tabular}




\section{LIST OF FIGURES}

FIGURE 1 Example of the scissor effect [source: Light Reading] ............ 17

FIGURE 2 D2D integrated cellular network for MTC ............................... 19

FIGURE 3 WCDMA/HSPA key performance indicators .......................... 24

FIGURE $4 \quad$ Illustration of VoIP traffic .......................................................... 26

FIGURE 5 E-Model rating as a function of Mouth-to-ear delay [ms] [IG114] ................................................................... 26

FIGURE 6 Noise Rise as a function of the inactive UE number and the DPCCH activity factor ............................................... 29

FIGURE $7 \quad$ Illustration of VoIP transmission on E-DCH assuming $10 \mathrm{~ms}$ TTI and bundling of two VoIP packets into one TTI with continuous and gated DPCCH transmission........... 31

FIGURE 8 3GPP protocol stack (see [Cun03]) ............................................. 33

FIGURE 9 D2D and cellular links (solid line) and interference couplings (dashed line) when D2D operation uses the uplink band of a cellular system with the frequency reuse factor of one and dedicated D2D resources within a cell ..................................................................... 41

FIGURE 10 Illustration of inband emissions............................................... 43

FIGURE $11 \quad$ Principle of PF scheduler [Hol11] ......................................... 44

FIGURE 12 UE scheduling activity vs. UE average performance (average SINR); Case 1 (ISD=500m), 25UEs/Cell (10 D2D UEs and 15 Cellular UEs)............................................. 45

FIGURE 13 Sequence diagram for dynamic mode switching ..................... 48

FIGURE $14 \quad$ Illustration of resource allocation .............................................. 52

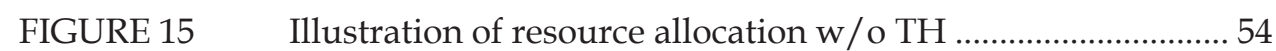

FIGURE 16 Derivation of a relative threshold for CQI reporting............... 55

FIGURE 17 Flow chart for enhanced scheduler with optimized D2D CQI reporting .............................................................. 56

FIGURE 18 Illustration of resource partition for D2D TDD channels within cellular FDD UL spectrum........................... 58

FIGURE 19 Asymmetric D2D communication between a D2D device and a legacy device.................................................... 59

FIGURE 20 Example of asymmetric D2D communication procedure with explicit mode selection signaling for legacy UE.....

FIGURE 21

HARQ feedback mapped to a common resource.

FIGURE 22 Cell layout with several D2D circles per cell, each D2D circle (cyan circle) including one Tx UE (blue circle) and one Rx UE (green circle). 


\section{LIST OF TABLES}

TABLE 1 Timestamp in IP header (see [Ric94]) ………………………....... 33

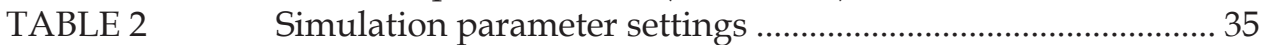

TABLE 3 Summary of simulation results ................................................ 36

TABLE $4 \quad$ Key components of Semi-autonomous D2D procedure.......... 57

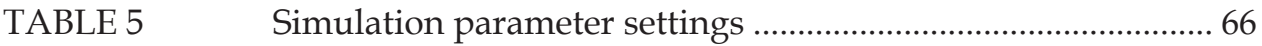

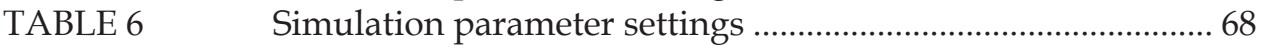

TABLE $7 \quad$ System capacity - Number of active links per cell .................... 68

TABLE $8 \quad$ Simulation parameter settings .................................................... 70

TABLE $9 \quad$ Simulation parameter settings ………………………….......... 72 


\title{
CONTENTS
}

\author{
ABSTRACT \\ ACKNOWLEDGEMENTS \\ ACRONYMS \\ LIST OF FIGURES \\ LIST OF TABLES \\ CONTENTS \\ LIST OF INCLUDED ARTICLES \\ GRANTED PATENTS
}





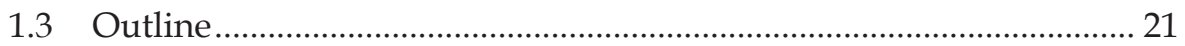

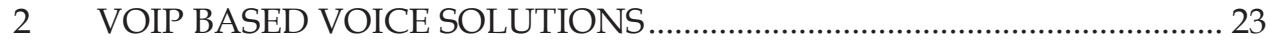

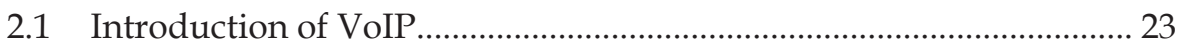

2.2 HSPA system description ..................................................................... 23

2.3 Properties and requirements for VoIP .................................................. 25

2.3.1 Adaptive Multi-Rate (AMR) .................................................... 25

2.3.2 Delay requirements..................................................................... 26

2.3.3 Quality criteria ........................................................................ 26

2.4 Control channel limitations for VoIP ................................................. 27

2.4.1 DPCCH overhead in the uplink transmission ........................... 28

2.4.2 Overhead from HS-DPCCH carrying CQI ................................. 29

2.4.3 HS-SCCH and E-DPCCH overhead associated with

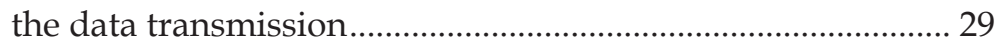



2.5.1 Robust Header Compression (ROHC) ....................................... 30

2.5.2 Uplink gating associated with the packet bundling .................... 30

2.5.3 HS-SCCH-less operation associated with the dynamic

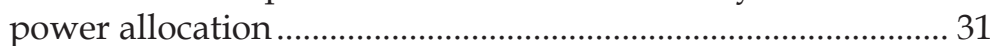

2.5.4 VoIP packet age aided HSDPA scheduling............................... 32

2.6 Performance evaluation for VoIP over HSPA ........................................ 34

2.6.1 Simulation methodology and assumptions ............................... 34

2.6.2 VoIP performance in HSPA .......................................................... 35

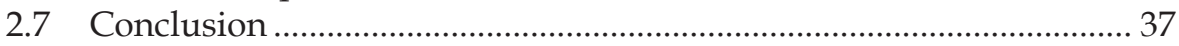

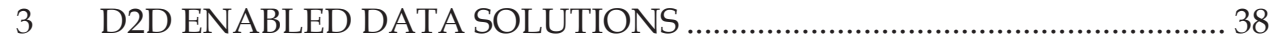

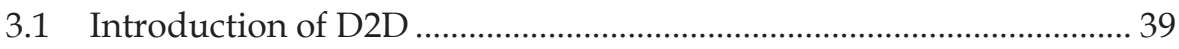

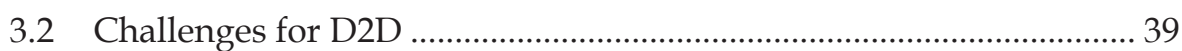


3.2.1 Impact of co-channel cross-link Interference ............................ 39

3.2.2 Impact of near-far interferer on ADC range............................... 42

3.2.3 Impact of inter-carrier interference (ICI) .................................. 43

3.2.4 Scheduling fairness with mixed D2D and cellular

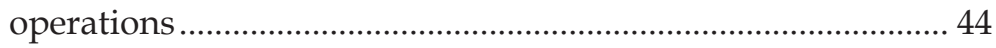

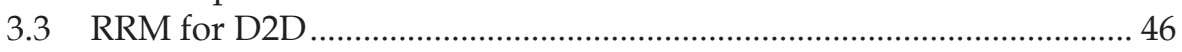

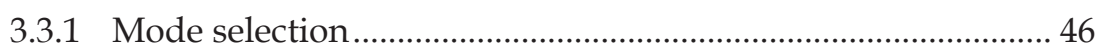

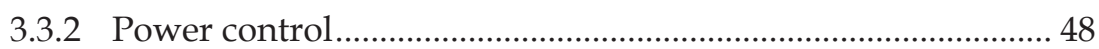

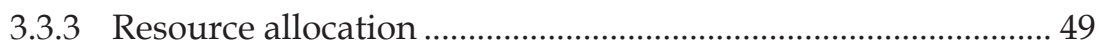

3.3.4 Time hopping for co-channel interference mitigation.............. 51

3.3.5 Enhanced scheduler with optimized D2D CQI

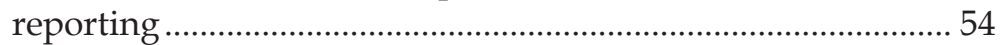

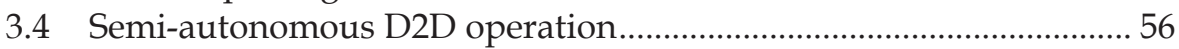

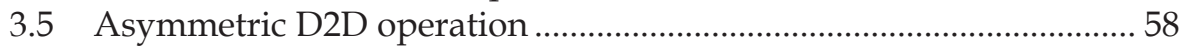

3.6 Feedback assisted D2D multicast operation ………............................... 63

3.6.1 HARQ Feedback on common resources ...................................... 64

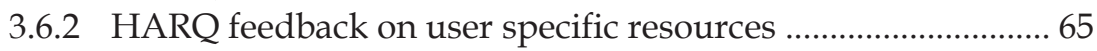



3.7.1 Simulation setup and performance analysis for RRM



3.7.1.1 Performance for the interference limited scenario ............ 67

3.7.1.2 Performance for the coverage limited scenario ................. 67

3.7.1.3 Performance for D2D mode selection.................................. 68

3.7.2 Simulation setup and results analysis for time

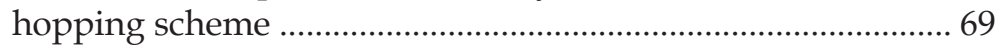

3.7.2.1 Effect of near-far interferers ................................................. 70

3.7.2.2 Effect of $\mathrm{TH}$ on the performance.......................................... 70

3.7.2.3 Effect of TH parameters on the performance..................... 71

3.7.3 Simulation setup and results analysis for semi-

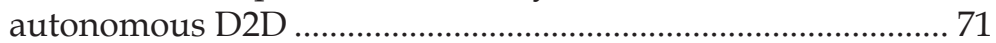

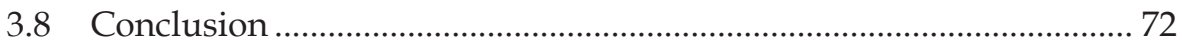

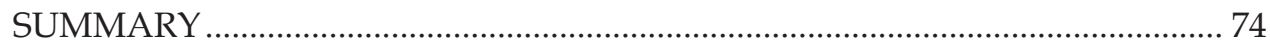

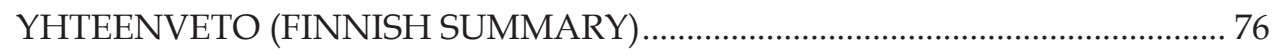

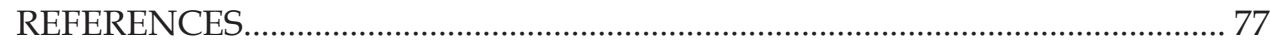

INCLUDED ARTICLES 


\section{LIST OF INCLUDED ARTICLES}

PI Chen, T., Kuusela, M., Malkamäki, E., “Uplink Capacity of VoIP on HSUPA", Proceedings of the 63rd IEEE Vehicular Technology Conference (VTC'06-spring), Melbourne, Australia, 2006.

PII Fresan, O., Chen, T., Malkamäki, E. and Ristaniemi, T., “DPCCH gating gain for voice over IP on HSUPA", Proceedings of IEEE Wireless Communications and Networking Conference 2007 (WCNC'07), Hong Kong, China, 2007.

PIII Chen, T., Malkamäki, E. and Ristaniemi, T., “Uplink DPCCH Gating of Inactive UEs in Continuous Packet Connectivity Mode for HSUPA", Proceedings of IEEE Wireless Communications and Networking Conference 2007 (WCNC'07), Hong Kong, China, 2007.

PIV Chen, T., Charbit, G., Ranta-aho, K., Fresan, O. and Ristaniemi, T., "VoIP End-to-End Performance in HSPA with Packet Age Aided HSDPA Scheduling", PIMRC 2008

PV Kuusela, M., Chen, T., Lundén, P., Wang, H., Henttonen, T., Ojala, J. and Malkamäki, E., "Radio Access Network VoIP Optimization and Performance on 3GPP HSPA/LTE “, Book Chapter of VoIP handbook, Applications, technologies, reliability and security. CRC press, 2009.

PVI Chen, T., Kunnari, E. and Ristaniemi, T., “Device-to-Device Communication for LTE-Advanced Network System", Report of the Department of Mathematical Information Technology, University of Jyväskylä, ISBN 978-951-39-5919-7, ISSN 1456-4386, 2014.

PVII Chen, T., Charbit, G. and Hakola, S., "Time Hopping for Device-to-device Communication in LTE Cellular System”, Proceedings of IEEE Wireless Communications and Networking Conference 2010 (WCNC'10), Sydney, Australia, 2010.

PVIII Xu, S., Wang, H., Chen, T., Peng, T., Kwak K.,S., “Device-to-Device Communication Underlaying Cellular Networks-Connection Establishment and Interference Avoidance", KSII TRANSACTIONS ON INTERNET AND INFORMATION SYSTEMS, TIIS 6(1): 203-228 (2012)

PIX Seppälä, J., Koskela, T., Chen, T. and Hakola, S., "Network Controlled Device-to-Device (D2D) and Cluster Multicast Concept for LTE and LTE-A Networks", Proceedings of IEEE Wireless Communications and Networking Conference 2011 (WCNC'11), Quintana-Roo, Mexico, 2011 
The author of this thesis was the main author of article PI for which he developed the system level simulator with system modeling and implementation, conducted all system simulations and performance analysis. In article PII the author was engaged to develop system modeling with part of the implementation work, conducted part of the system simulations and performance analysis. As the main author of article PIII, the author of this thesis did the modeling and implementation work, conducted all system simulations and performance analysis. In article PIV, the author was the main author that developed the concept and system modeling as well as conducted all system simulations and performance analysis. As the main contributor of article PV, the author was responsible for the overall contents of the chapter, especially focusing on the sections relevant to VoIP over HSUPA. In article PVI, the author developed the concept, gave part of the system modeling, and conducted the system simulations and performance analysis. For article PVII, the author participated in developing the concept, implemented system modeling, and conducted all system simulations and performance analysis. In article PVIII, the author contributed for the part of concept development and system simulation modeling, and conducted some performance analysis. For article PIX, the author mainly contributed to the concept development and system design. 


\section{GRANTED PATENTS}

1. Chen, T., Wang H. and Peng, T., "Method, apparatus and computer program product for allocating resources in wireless communication network", US Pat. No. 8,885,507.

2. Chen, T., "Method and apparatus for unlicensed band operation", US Pat. No. 8,879,576.

3. Koskela, T., Chen, T. and Hakola, S., “Network controlled device to device / machine to machine cluster operation", US Pat. No. 8,867,458.

4. Chen, T., Callender, C., P., Lehti, A. and Koul, S., "Interference avoidance in low power nodes", US Pat. No. 8,861,412.

5. Chen, T. and Charbit, G., "Low-cost LTE system with distributed carrier aggregation on the unlicensed band", US Pat. No. 8,837,422.

6. Charbit, G. and Chen, T., "Finding mobile station for device-to-device communication", US Pat. No. 8,818,861.

7. Charbit, G., Chen, T., Hakola, S., and Koskela, T., "Synchronization for device-to-device communication", US Pat. No. 8,811,374.

8. Chen, T. and Lu, Q. "Autonomous unlicensed band reuse in mixed cellular and device-to-device network", US Pat. No. 8,792,900.

9. Charbit, G., Chen, T., Hakola, S. and Koskela, T., "Apparatus and method of scheduling resources for device-to-device communications", US Pat. No. 8,761,099.

10. Charbit, G. and Chen, T., "Frequency hopping in licenseexempt/shared bands", US Pat. No. 8,675,605.

11. Chen, T. and Kunnari, E., "Method and apparatus for facilitating packet scheduling for a hybrid communication network", US Pat. No. 8,675,580.

12. Yu, L., Van, P., V., Horneman, K., V., Vesterinen, S., I., Koskela, T. and Chen, T., "Systems, methods, and apparatuses for facilitating deviceto-device connection establishment", US Pat. No. 8,666,403.

13. Chen, T. and Charbit, G., "Wireless communication systems and methods", US Pat. No. 8,626,205.

14. Chen, T., Hakola, S. and Koskela, T., "Multicast transmission within a hybrid direct and cellular communication system", US Pat. No. $8,582,593$.

15. Chen, T. and Lu, Q., “Apparatus and method employing scheduler behavior aware predictive resource selection in a communication system", US Pat. No. 8,401,562.

16. Callender, C., P. and Chen, T., “Test method for type 3I receiver in multicarrier configuration", US Pat. No. 8,331,261.

17. Koskela, T. and Chen, T., "Apparatus and method for flexible switching between device-to-device communication mode and cellular communication mode", US Pat. No. 8,213,360. 



\section{INTRODUCTION}

In the future, one big challenge for mobile operators to face is the scissor effect, i.e., revenue growth will not keep up with the traffic growth (see [Gre10]), as illustrated in FIGURE 1. In other words, operators have to ensure that the growing infrastructure investment can fulfill the demands of the exponential growth of mobile traffic while keeping the proportional increase in revenue. The increasing demand for the cellular network to offer diverse applications with low operation costs for busting the myth of the scissor effect has been speeding up the evolution of the cellular network towards the more flat network architecture. More specifically, the advanced technical solutions are envisioned to offer two types of conventional services more efficiently: voice service and data service, which would be significant for the success of the mobile broadband business in the way of the cellular network evolution.

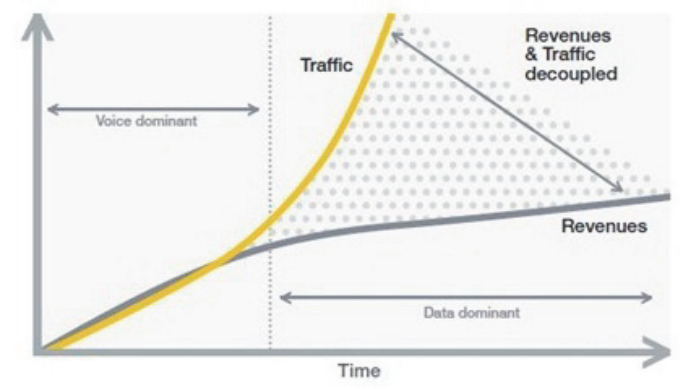

FIGURE 1 Example of the scissor effect [source: Light Reading]

For voice service, it is characterized by the high demand on the packet delay and the small size of the payload, which has been typically carried by the dedicated radio resources and transported through the circuit switched core network to secure the quality of service. However, recently there has been an increasing interest in using cellular networks for real-time (RT) packet switched (PS) services such as Voice over IP (VoIP). The reason behind the increased interest towards VoIP is to use VoIP in All-IP networks instead of using circuit 
switched (CS) speech (see [AT009]). This would result in cost savings for operators as the CS related part of the core network would not be needed anymore. In conventional cellular networks, RT services (e.g. voice) are carried over dedicated channels because of their delay sensitivity, while Non-Real Time (NRT) services (e.g. web browsing) are typically transported over time-shared channels because of their burstiness and lower sensitivity to delay. However, with careful design of the system, RT services can be efficiently transported over time-shared channels as well. A potential advantage of transmission speech on a channel previously designed for data traffic is the improved efficiency in terms of resource sharing, spectrum usage, provision of multimedia services and network architecture. The challenge is to port VoIP services on wireless networks while retaining the QoS of circuit-switched networks and the inherent flexibility of IP-based services. Thus, the further improvement of the radio network to support VoIP services efficiently is required for cellular system evolution.

On the other hand, to effectively mitigate the scissor effect caused by the data dominant services, the high efficient cellular networks and value-added services are critical for the operators. Long Term Evolution (LTE) with the peak data rates of downlink (DL) 100Mpbs and uplink (UL) 50Mbps has been in 3GPP (e.g., [4G300]) to fulfill the requirements of fast increasing mobile traffic, which can significantly improve the spectrum efficiency and reduce transmission latency. Further, a hybrid network integrating device-to-device (D2D) communication into a LTE network is considered to further improve the networks efficiency and enable some new value-added services, in which the D2D devices (or mobiles or terminals or peers or machines) with LTE capability having a direct physical communication link can reuse LTE air interface for the local peer-to-peer traffic while sharing the cellular network resources with devices having a conventional cellular connection to the base station (a.k.a. eNB in LTE) for the exterior traffic. For example, the cellular network operates in frequency division duplex (FDD) mode in which the D2D connections utilize the time division duplex (TDD) mode either using cellular network UL and/or DL resources controlled by the base station(s). The general concept covering FDD or TDD cellular network where a direct communication connection utilizes either the FDD or TDD duplex mode can be found from [Mcl05]. The purpose of adding direct communication into a cellular network is the possibility to reduce transmitter power consumption both in the device and the base station, increase cellular network capacity and establishing more services for the users, which is pointed out in [Alc08]. Different than the mobile relaying based system as mostly studied (e.g., in [Hsi02] and [Lap08]), the D2D connection under the coverage of the cellular network is mainly established for carrying the local peer-to-peer direct traffic with one-hop transmission, which accordingly may require different gain mechanisms and resource allocation schemes. The D2D integrated hybrid network is mainly targeting to offload the peer-to-peer traffic from the transportation network and reduce the transmission delay bypassing the access network. On the other hand, a D2D integrated hybrid cellular system may pave 
the way for D2D based wireless-mesh networks in the cellular system, in which D2D devices are pivotal in offering cost-effective transportation services for the devices of machine type communications (MTC). As illustrated in FIGURE 2, D2D devices can act as a machine gateway to forward the data from wireless sensors for coverage extension, exchange information between each other for coordination, and communicate with the base station when offering a service. In short, D2D devices can play several critical roles in the future networks for more efficient cellular system operations.

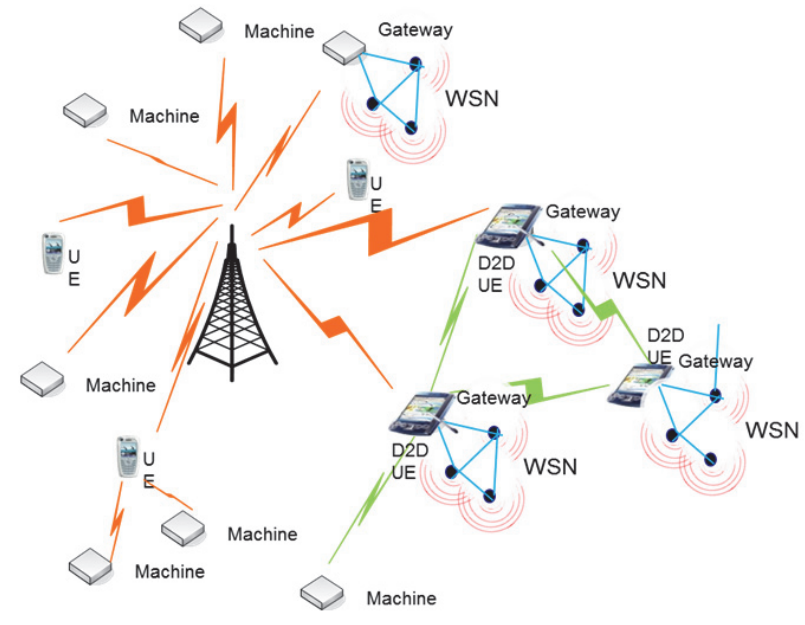

FIGURE 2 D2D integrated cellular network for MTC

Due to the challenges from the interference environment in this kind of hybrid network supporting both cellular and D2D communication, it is important for radio resource management (RRM) to ensure the quality of service (QoS) and leverage the performance of cellular and D2D communication when sharing the same radio resources. In addition, to fully exploit the potential gain from the spatial reuse between D2D links (and cellular links) for improving the spectrum efficiency significantly, the co-channel interference from the reused resources should be addressed carefully. Otherwise, the imperfect spatial reuse may even degrade the systems performance.

In general, in order to bust the myth of the scissor effect, the VoIP based voice service solution and D2D based data service solution can be considered as potential candidates for future network evolution.

\subsection{Research problems}

Voice over IP is a technology which has been widely used in the wired network to provide call services in a cost-effective way. However, porting VoIP over the wireless system like the conventional cellular system is not so straightforward. More specifically, there are some challenges in the radio access network to sup- 
port VoIP based RT traffic, such as packet delay, packet jitter and transportation overhead. Thus, the first question to be answered in this thesis is:

Q1: What are the challenges and constraints for VoIP services over cellular network?

The answers to Q1 will give the hints for system design in which the potential solutions can focus on removing such constraints to improve the performance. Any feasible solution to address Q2 should be justified with thorough investigation, for which the extensive simulations should be conducted for performance evaluation. Thus, different quality criteria and deployment scenarios have been used for evaluation with the proper system modeling.

Q2: How to improve the cellular system for offering the VoIP services more efficiently?

For D2D based data solutions, as studied for VoIP based voice solution, it is important to firstly do an analysis on the D2D operation scenarios and find the challenges for the D2D integrated cellular system. For this purpose, Q3 for the analysis of the bottleneck of the D2D system is raised for discussion in this thesis:

Q3: What are the challenges for D2D operations under the cellular system?

Secondly, the potential solutions for improving D2D operations are proposed in the thesis to address the problems observed from the analysis. With the answers to Q4, the difference on RRM components for a hybrid system than the conventional centralized cellular system can be noticed.

Q4: What are the RRM solutions for the feasible D2D operation under the cellular system?

Further, there can be different ways of operating D2D communications depending on the deployment scenarios and applications, which will be analyzed to answer Q5 .

Q5: How to support the unicast and/or multicast data services via D2D communications effectively with a decent performance gain?

Moreover, simulation results are presented for D2D evaluation.

With the answers for the abovementioned five questions in this thesis, it is expected to prove that VoIP based voice solutions and D2D based data solutions can bust the myth of the scissor effect and provide cost-effective solutions for future network operation.

\subsection{Other articles}

In addition to the included articles, the author of this thesis has also published several articles as below: 
1. Holma Harri, Markku Kuusela, Esa Malkamäki, Karri Ranta-aho \& Tao Chen, "VoIP over HSPA with 3GPP Release 7", PIMRC 2006

2. S. Hakola, T. Chen, J. Lehtomäki and T. Koskela, "Device-to-Device (D2D) Communication in Cellular Network - Performance Analysis of Optimum and Practical Communication Mode Selection", Proceedings of IEEE Wireless Communications and Networking Conference 2010 (WCNC'10), Sydney, Australia, 2010.

3. T. Koskela, S. Hakola, T. Chen and J. Lehtomäki, "Clustering Concept using Device-to-Device Communication in Cellular System", Proceedings of IEEE Wireless Communications and Networking Conference 2010 (WCNC'10), Sydney, Australia, 2010.

4. Shaoyi Xu, Jing Han, Tao Chen, "Enhanced Inter-Cell Interference Coordination in Heterogeneous Networks for LTE-Advanced", VTC Spring 2012.

5. Shaoyi $\mathrm{Xu}$, Haiming Wang, Tao Chen, "Effective Interference Cancellation Mechanisms for D2D Communication in Multi-Cell Cellular Networks", VTC Spring 2012.

6. Shaoyi $\mathrm{Xu}$, Haiming Wang, Tao Chen, Qing Huang, Tao Peng, "Effective Interference Cancellation Scheme for Device-to-Device Communication Underlaying Cellular Networks", VTC Fall 2010

7. Muller, A., Tao Chen, "HSDPA Link Adaptation by Considering the Age of Channel Quality Feedback Information", VTC Fall 2005

8. Jianke Fan, Tao Chen, "Packet Scheduling Algorithms for Mixed Traffic Flow over HSDPA", PIMRC 2007

\subsection{Outline}

In short, in chapter 2 and chapter 3, we investigated the VoIP based voice solutions and D2D based data solutions separately. The summary was finally presented in chapter 4 .

More specifically, in chapter 2, a short introduction for VoIP is presented in Section 2.1, which is followed by a brief description of a target cellular system under study in Section 2.2. Then the properties and relevant QoS requirements for VoIP are discussed in Section 2.3 for preparation of the system design. The limitation or constraints, which may prevent the application of VoIP over the cellular network, are investigated in Section 2.4. Based on the conducted analysis, the cellular VoIP based solutions have been proposed to offer the same quality of voice services in a more cost-effective approach, which are discussed in Section 2.5. The performance has been evaluated with extensive simulations in Section 2.6 and the conclusions are drawn in Section 2.7.

In chapter 3, an introduction for D2D under study is presented first in Section 3.1. Challenges for D2D operation under LTE network are analyzed in Section 3.2. Then RRM solutions to address the challenges and improve the D2D performance are described in Section 3.3. To reduce the signaling overhead, the 
semi-autonomous D2D is proposed and presented in Section 3.4. In addition, different than the conventional symmetric D2D operation, asymmetric D2D operation is proposed for consideration in the system design as discussed in Section 3.5. Considering the increasing interest in the D2D cluster based multicast service, the feedback assisted D2D multicast is discussed in Section 3.6, which also provides some hints for the long term evolutions of the D2D system. Finally, the performance has been investigated with extensive system simulations in Section 3.7 for the various solutions. The conclusions are finally given in Section 3.8. 


\section{VOIP BASED VOICE SOLUTIONS}

\subsection{Introduction of VoIP}

The initial research in analyzing VoIP performance over wireless channels has mostly focused on wireless local area networks (e.g. IEEE 802.11 WLAN). Besides that, the VoIP service is also supported by a wide area of cellular networks (e.g. HSPA and E-UTRAN) which is due to its integrated IP infrastructure and larger user capacity than traditional voice of circuit switched mode.

High-speed Uplink Packet Access (HSUPA), which is included in 3GPP Release 6 specification (see, e.g., [3G309],[3G896]), has been designed to increase the uplink packet data throughput by means of a Hybrid Automatic Repeat request (HARQ), shorter frame size and fast scheduling controlled by the base station. High Speed Downlink Packet Access (HSDPA) is the downlink counterpart of HSUPA in 3GPP Release 5 (see, e.g., [3G308]). Furthermore, both HSUPA and HSDPA, which are so called High-speed Packet Access (HSPA), have been further improved in Release 7 . The challenge for the VoIP on HSPA would be the delay requirement for the voice traffic, and the small packet sizes as well which makes it difficult to efficiently utilize the bandwidth due to control channel constraints.

This chapter aims to give an overview of the challenges faced in implementing VoIP service over packet switched centralized cellular networks, in particular 3GPP HSPA. Generally accepted solutions leading to an efficient overall VoIP concept are outlined and the performance impact of various aspects of the concept is addressed. The chapter is concluded with a system simulation based performance analysis of VoIP service.

\subsection{HSPA system description}

HSPA, a 3GPP standardized evolution of Wideband Code Division Multiple Access (WCDMA), has succeeded in becoming the world's leading third generation mobile standard. HSPA consists of both HSUPA and HSDPA which en- 
hance WCDMA in the uplink and the downlink separately. Several key techniques such as HARQ, fast scheduling controlled by the base station and the shorter frame size are introduced into HSPA targeting higher data rates with more efficient spectrum usage and a lower transmission delay (See FIGURE 3).

In HSDPA, a high-speed downlink shared channel (HS-DSCH) is specifically associated with $2 \mathrm{~ms}$ frame size, in which the users would share at most 15 High-Speed physical downlink shared channels (HS-PDSCHs) with the fixed spreading factor 16 (SF 16) by code multiplexing. In this way, the radio resource would be utilized in a more efficient way than early dedicated resource allocation in WCDMA without considering the burstiness of the traffic. Otherwise, HARQ in HSPA can provide the physical layer (L1) re-transmissions and soft combining, which would avoid a large amount of higher layer ARQ transmission and frame selection (i.e., hard combining). This also improves the efficiency of the Iub interface between the base station and radio network controller (RNC) significantly. $2 \mathrm{~ms}$ in addition to $10 \mathrm{~ms}$ TTI frame length introduced into HSUPA can further reduce the transmission delay in the air interface. Thus, the Round Trip Time (RTT) can be decreased from about $150 \mathrm{~ms}$ in WCDMA to 50 ms in HSPA. Besides, base station controlled fast scheduling can select the user in a relative good channel condition for the transmission reaching the higher throughput due to the multi-user diversity gain. This is impossible in the WCDMA system, where the reaction of the RNC controlled scheduling to the varying channel condition is too slow to follow the fast changing channel condition because of the huge transmission delay between UE and the RNC via the base station.

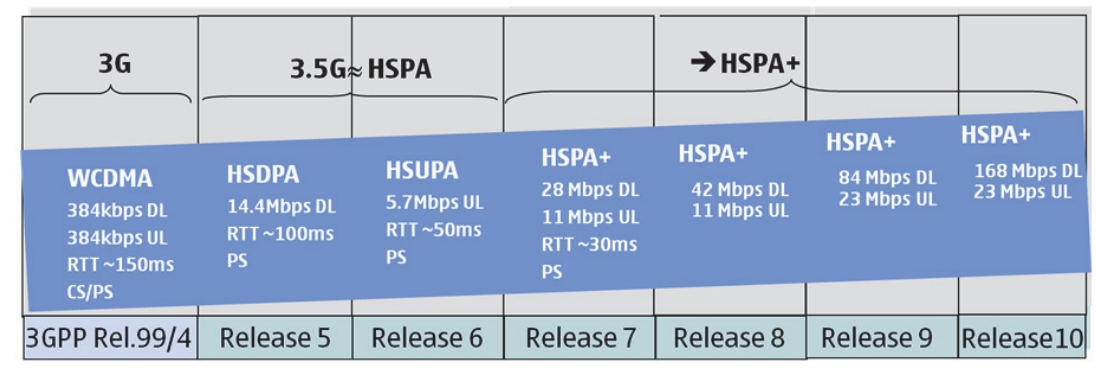

FIGURE 3 WCDMA/HSPA key performance indicators

In particular, in the context of VoIP, there are two scheduling schemes defined for HSUPA: Node B controlled scheduling transmission and RNC controlled non-scheduled transmission. However, the former scheme does not guarantee a minimum bit rate since the scheduling algorithm decides whether to allocate any power to the user or not. On the other hand, the non-scheduled transmission scheme defines a minimum data rate at which the UE can transmit without any previous request. When a non-scheduled transmission is configured by the SRNC, the UE is allowed to send E-DCH data at any time, up to a configured bitrate, without receiving any scheduling command from the Node B. Thus, signaling overhead and scheduling delay are minimized. Therefore, 
RNC controlled non-scheduled transmission is the most suitable choice for VoIP traffic.

\subsection{Properties and requirements for VoIP}

In this section characteristics of VoIP traffic are given by describing the properties and functionality of the used voice codec in HSPA/LTE (Section 2.3.1) and by presenting the requirements and the used quality criteria for VoIP traffic in Sections 2.3.2 and 2.3.3, respectively.

\subsubsection{Adaptive Multi-Rate (AMR)}

Adaptive Multi-Rate (AMR) is an audio data compression scheme optimized for speech coding. AMR was adopted as the standard speech codec by 3GPP in October 1998 and is now widely used. The AMR speech coder consists of a multi-rate speech coder, a source controlled rate scheme including a voice activity detector and a comfort noise generation system, and an error concealment mechanism to combat the effects of transmission errors and lost packets.

The multi-rate speech coder is a single integrated speech codec with eight source rates from $4.75 \mathrm{kbps}$ to $12.2 \mathrm{kbps}$, and a low rate background noise encoding mode. For AMR the sampling rate is $8 \mathrm{kHz}$. The usage of AMR requires optimized link adaptation that selects the best codec mode to meet the local radio channel and capacity requirements. If the radio conditions are bad, source coding is reduced and channel coding is increased.

In addition to AMR audio codec, an extension of it is abbreviated as AMRWB (wideband) audio codec that extends AMR. The sampling rate for AMRWB is $16 \mathrm{kHz}$, which is double the sampling rate of AMR. Therefore AMR is often abbreviated as AMR-NB (narrowband). AMR-WB shall be supported if 16 kbps sampling for the audio is used in the UE. Similarly like AMR-NB, AMRWB operates with various bit rates. For AMR-WB the bit rates range from 6.60 kbps to $23.85 \mathrm{kbps}$. It is emphasized, that both AMR-NB and AMR-WB are used in HSPA and LTE radio systems. A more detailed description of AMR-NB and AMR-WB can be found in [3G071].

Functionality of the AMR codec is illustrated in FIGURE 4. During voice activity periods there is one voice frame generated every $20 \mathrm{~ms}$ (the number of bits/frame depends on the AMR codec mode) while during the silent periods Silence Insertion Descriptor (SID) frames are generated once in every $160 \mathrm{~ms}$. 


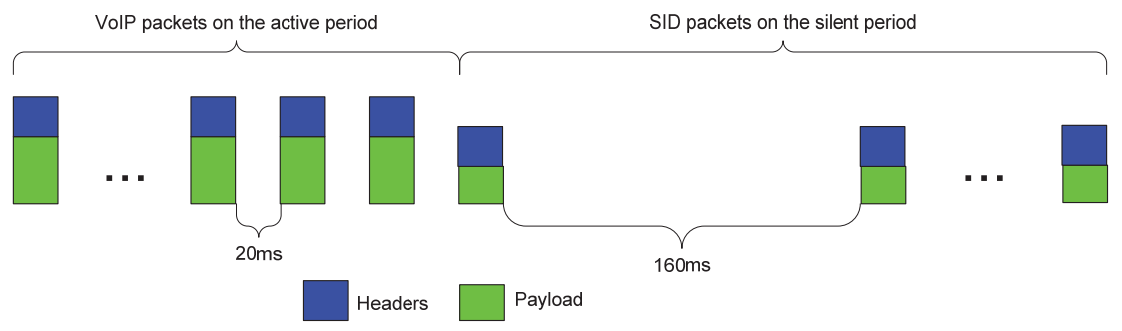

FIGURE 4 Illustration of VoIP traffic

\subsubsection{Delay requirements}

VoIP is a conversational class service and thus the packet delay should be strictly maintained under some reasonable limits. According to the maximum acceptable mouth-to-ear delay for voice is in the order of $250 \mathrm{~ms}$, as illustrated in FIGURE 5. Assuming that the delay for the core network is approximately 100 $\mathrm{ms}$, the tolerable delay for MAC buffering/scheduling and detection should be strictly below $150 \mathrm{~ms}$. Hence, assuming that both end users are (E-)UTRAN users, the tolerable one way delay for MAC buffering and scheduling should be under $80 \mathrm{~ms}$. For HSPA the HARQ RTT for HSPA would be about $16 \mathrm{~ms}$ with 2 ms TTI, which means at most 3 HARQ re-transmissions supposing the airinterface-delay of $50 \mathrm{~ms}$ is used. Similarly, it would be at most 1 HARQ retransmission corresponding to 40ms HARQ RTT with 10ms TTI in the uplink.

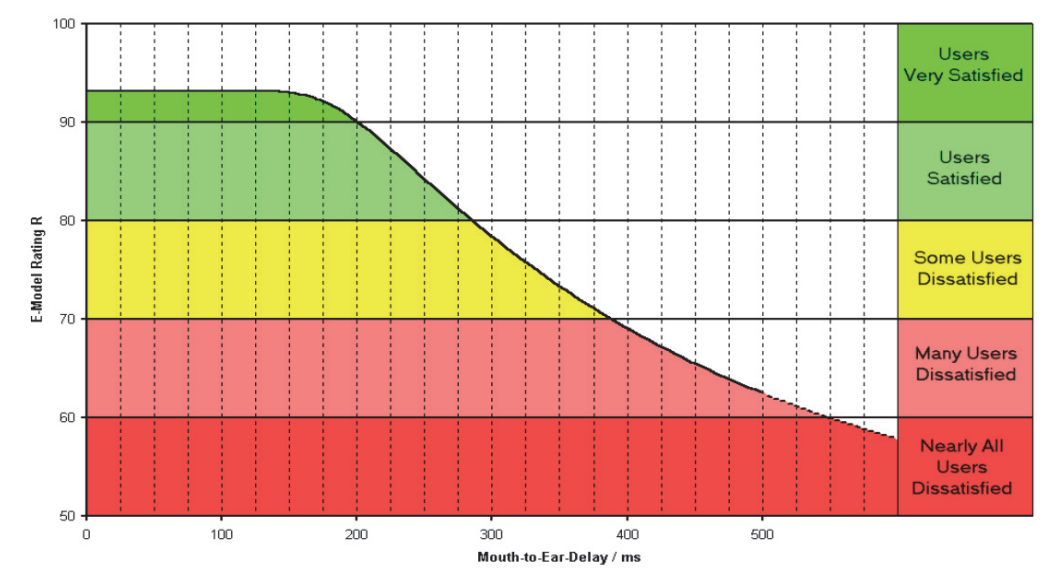

FIGURE 5 E-Model rating as a function of Mouth-to-ear delay [ms] [IG114]

\subsubsection{Quality criteria}

Due to the nature of radio communication it is not practical to aim for $100 \%$ reliability when receiving all the VoIP packets in time. Instead, some degree of missing packets can be tolerated without notably affecting the quality of service perceived by the users. For VoIP traffic the system capacity is measured as the maximum number of users that could be supported without exceeding $5 \%$ out- 
age. During the standardization phases of HSPA in 3GPP, the outage criteria was used when evaluating the VoIP systems performance. Used outage criteria defines the system-wisely as follows. For HSPA, a user is in outage if at least $5 \%$ of the packets are not received within the delay budget when monitored over a 10 second window.

In the uplink (UL) direction there also exists an alternative method to measure VoIP performance, which is used in the context of this chapter for HSPA, i.e., UL VoIP capacity can be measured as the allowed number of users in a cell with an average noise rise of $\mathrm{X} \mathrm{dB}$ measured at the base station. Optimal value for the $X$ may depend on the used system, and for HSPA the used value was $6 \mathrm{~dB}$. It is emphasized, that even though the used outage criteria differ slightly for evaluation of VoIP over HSUPA, the performance difference originating from different outage criteria is rather minor being inside the error margins of the simulations as illustrated in Section 2.6.2.

\subsection{Control channel limitations for VoIP}

With VoIP traffic the number of supported users may become rather high. To support VoIP transmission of the downlink and uplink transport channels, there is a need for certain associated downlink and uplink control signaling. Therefore the overhead from associated control signaling in the uplink and downlink may become a bottleneck for the VoIP systems performance.

For VoIP traffic, the packet size is quite small corresponding to a quite low data rate transmission on the data channel, e.g., 32kbps with $10 \mathrm{~ms}$ TTI and $160 \mathrm{kbps}$ with $2 \mathrm{~ms}$ TTI in HSUPA. In other words, it implies the overhead from the control channels would consume relatively more power for the transmission of VoIP traffic than the other packet data traffics transmitted with the high data rate. On the other hand, the control channels cannot be subject to HARQ retransmissions, i.e., the information carried by the control channels cannot be soft combined. Thus, more retransmissions may lead to the higher overhead from the control channels associated with the data transmission. So it makes sense to study the effect of the overhead from the control channels on the performance and reduce them as much as possible.

In HSPA, there are mainly three types of dedicated physical control channels in the uplink and one in the downlink: the uplink Dedicated Physical Control Channel (uplink DPCCH), the E-DCH Dedicated Physical Control Channel (uplink E-DPCCH), the uplink Dedicated Control Channel associated with HSDSCH transmission (uplink HS-DPCCH) and the downlink Shared Control Channel for HS-DSCH (HS-SCCH). Their constraints on the VoIP performance will be addressed in the following sections. 


\subsubsection{DPCCH overhead in the uplink transmission}

The uplink DPCCH in 3GPP Release 6, i.e., HSUPA, is used to carry physical layer control information which mainly consists of known pilot bits to support channel estimation for coherent detection and transmit power-control (TPC) commands to be used for the downlink transmission. When the high capacity (in terms of users in a cell) is targeted for VoIP on HSDPA and HSUPA, then the interference caused by continuously transmitted uplink DPCCHs becomes the limiting factor for capacity.

To illustrate this, let us calculate the uplink capacity by using the following uplink load Eq 1

$$
N R_{d B}=-10 \log 10\left(1-\frac{\rho}{W / R} N \cdot v \cdot(1+i)\right)
$$

where $\rho$ is the EbN0 target after antenna combining, $W$ is the chip rate, $R$ is the E-DPDCH bit rate, $N$ is the number of users, $v$ is the equivalent activity factor, $i$ is the other cell to own cell interference ratio, and $N R_{d B}$ is the noise rise in decibels. When the uplink load Eq 1 is used, the overhead from DPCCH, E$\mathrm{DPCCH}$ and the re-transmissions are included in the equivalent activity factor $v$.

Further, it is also possible to divide this formula into components and then have separate Ec/No requirements and activity factors for each channel. Here E-DCH is assumed to include both E-DPCCH and E-DPDCH, which would always be transmitted together thus having the same activity factor:

$N R_{d B}=-10 \log 10\left(1-\left[\left(\frac{E_{c}}{N_{0}}\right)_{E-D C H} v_{E-D C H}+\left(\frac{E_{c}}{N_{0}}\right)_{D P C C H} v_{D P C C H}+\left(\frac{E_{c}}{N_{0}}\right)_{H S-D P C C H} v_{H S-D P C C H}\right] N \cdot(1+i)\right), \quad$ Eq 2

Here each channel was considered separately, i.e., the required Ec/No and the activity were defined separately for each channel. For DPCCH gating it was assumed that E-DPDCH and HS-DPCCH transmissions never overlap and thus the DPCCH activity was obtained as the sum of activities on E-DPDCH and HS$\mathrm{DPCCH}$. This assumption is a little bit pessimistic.

Thus, the Noise Rise (NR) contributed by inactive UEs (i.e., UEs with inactive packet transmission) can be calculated as shown in FIGURE 6 assuming that $i$ is 0.65 for the typical 3-sector macro cell scenario and the required $E c / N o$ is about $-18 \mathrm{~dB}$ for the inactive UE. Obviously, up to 30 inactive users with the continuous DPCCH transmission in this case can eat up all the reserved radio resource, i.e., $6 \mathrm{~dB}$ noise rise target. On the other hand, less DPCCH activity or less inactive UE number can reduce the uplink interference dramatically.

With VoIP over Enhanced Dedicated Channel (E-DCH), the uplink transmission of the voice is not continuous as the Circuit Switched (CS) voice over Dedicated Channel (DCH). One voice frame is transmitted every $20 \mathrm{~ms}$ or two voice frames every $40 \mathrm{~ms}$ if bundling of two voice packets is allowed. With VoIP, the continuous transmission of the uplink control channel DPCCH would consume the scarce radio resource and become inefficient. 


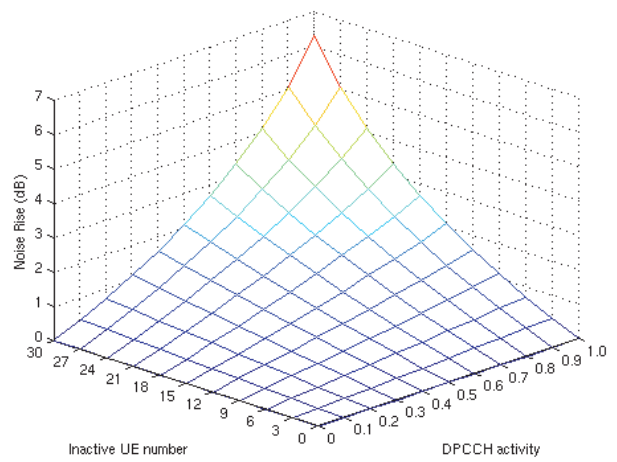

FIGURE 6 Noise Rise as a function of the inactive UE number and the DPCCH activity factor

\subsubsection{Overhead from HS-DPCCH carrying CQI}

Except for DPCCH channel, there are some other control channels such as HSDPCCH which may consume the uplink radio resource. HS-DPCCH, carrying Channel Quality Information (CQI) for downlink channel sensitive schedulers and adaptive modulation scheme selections, may transmit periodically according to a CQI reporting period controlled by a parameter. Correspondingly, the effect of the CQI reporting period can be studied according to the above equations as well. Reducing CQI activity (i.e., increasing CQI reporting period) from once per $10 \mathrm{~ms}$ to once per $20 \mathrm{~ms}$ increases the VoIP capacity about $10 \%$ and going down to one CQI transmission per $80 \mathrm{~ms}$ increases the VoIP capacity by 20\% (from CQI once per $10 \mathrm{~ms}$ ). On the other hand, the larger transmission interval of CQI may make the downlink performance worse due to less reliability of the CQI. Therefore, the CQI reporting period should be leveraged for the uplink and downlink performance.

\subsubsection{HS-SCCH and E-DPCCH overhead associated with the data transmis- sion}

Other control channel constraints for VoIP over HSPA would be the overhead from the VoIP packet transmission associated control channels such as HSSCCHs in the downlink and E-DPCCHs in the uplink. The HS-SCCH is a downlink physical channel used to carry downlink signaling related to HS-DSCH transmission, whereas the E-DPCCH is a physical channel used to transmit control information associated with the E-DCH. When a large number of users are served at a time, the control overhead (i.e. HS-SCCH overhead and E-DPCCH overhead) increases and the respective share of resources for the VoIP packet transmission of the user is reduced. Otherwise, more HARQ retransmissions would also increase these overhead. Thus, there would be a tradeoff between the allowed retransmission number and the control overhead. 


\subsection{Solutions for VoIP over HSPA}

To address the practical constraints for VoIP over HSPA, several solutions have been proposed to improve the VoIP performance.

\subsubsection{Robust Header Compression (ROHC)}

In streaming applications like VoIP, the overhead from IP, UDP and RTP headers is 46 bytes for IPv4, and 66 bytes for IPv6. For VoIP traffic this corresponds to around 144-206 \% header overhead, assuming that the most typical VoIP codec (AMR $12.2 \mathrm{kbps}$ ) is used with a 32 byte payload. Such a large overhead is excessive in wireless systems due to the limited bandwidth and would lead to serious degradations in the spectrum efficiency.

In order to overcome this, a robust header compression (ROHC) technique was adopted as a non-mandatory feature to 3GPP specifications from Release 04 onwards - hence ROHC feature is supported in HSPA and LTE radio systems as well. With ROHC the IP/RTP/UDP header is compressed down to few bytes the minimum size being 3 bytes, which is also the most commonly used header size. Hence with ROHC the header overhead may be compressed down to $25 \%$, implying that the utilization of $\mathrm{ROHC}$ leads to significant improvements in the VoIP air interface capacity.

\subsubsection{Uplink gating associated with the packet bundling}

To reduce the overhead of control channels, packet bundling is adopted and studied not only in HSPA but also other wireless networks. Earlier, circuit switched voice transmits 1 voice packet per $20 \mathrm{~ms}$ radio frame using the convolutional code. Packet bundling for VoIP over HSPA is used by sending multiple VoIP packets together, in which Turbo code can be applied and performs more effectively. Furthermore, it can minimize the associated control overhead improving the performance in both the uplink and downlink. Packet bundling is an attractive method to reduce control channel.

In 3GPP Releases preceding Release 6, DPCCH channel was transmitted continuously regardless of whether there is actual user data to be transmitted or not, thus highly loading the cell as illustrated in FIGURE 6. The idea in gating, which is introduced in 3GPP Release 7 presented in [3G903] is to stop the transmission of DPCCH when there is no data to be sent on E-DCH and no L1 feedback signaling on HS-DPCCH, see FIGURE 7. Therefore, the interference to other users would be reduced, and increase the uplink capacity. Besides, it can increase UE standby time efficiently because of less power consumption. 




FIGURE 7 Illustration of VoIP transmission on E-DCH assuming $10 \mathrm{~ms}$ TTI and bundling of two VoIP packets into one TTI with continuous and gated DPCCH transmission.

Due to DPCCH gating, it might be difficult for the network to distinguish an inactive period from a loose connection. That is why the DPCCH transmission is desirable not to be totally silent but transmitted periodically following a predefined pattern. Another issue to consider is the transmission power. After a gating period without tracking the received SINR on DPCCH, the channel response variations could degrade the transmission and increase the interference between the UEs. Thus, in the 3GPP specifications for DPCCH gating the optional power control preambles are defined to be sent previous to the data channel reactivation.

More details have also been discussed in article PI, PII and PIII.

\subsubsection{HS-SCCH-less operation associated with the dynamic power allocation}

3GPP Release 7 also allows HS-SCCH-less operation in the downlink to minimize the interference from HS-SCCH. The high capacity VoIP typically requires 4-6 code multiplexed users in the downlink of Release 6 . Each code multiplexed user requires a HS-SCCH channel. HS-SCCH-less operation can be applied to VoIP when the packet inter-arrival time is constant. In brief, the principle of HS$\mathrm{SCCH}-$ less operation is to skip the associated HS-SCCH transmission for the initial transmission of the VoIP packet, supposing the UE can instantly predict the VoIP packet arrival based on $20 \mathrm{~ms}$ inter-arrival time and blindly detect VoIP transmission format limited by a few options due to the small packet size. However, the HS-SCCH transmission associated with retransmission of VoIP packets should remain to provide the flexibility on the asynchronous scheduling and opportunities for link adaption during the retransmission phase. Thus, the interference from HS-SCCH transmissions can be reduced with HS-SCCHless operation leading to higher VoIP capacity.

Otherwise, the dynamic HS-DSCH power allocation on HS-PDSCH and HS-SCCH channels in the case of HS-SCCH-less operation for VoIP service enables more support than 4 code-multiplexed users on HS-DSCH due to a more efficient use of the scarce power resource. This improves performance especially with the short 50ms delay requirement, as buffering of VoIP packets in MAC$\mathrm{hs}$ is becoming very restricted leading to higher power requirements. Especially in case of mixed traffic with both VoIP and data services, the scarce power resource spared by the HS-SCCH-less operation can turn to a higher throughput 
for the data service by means of advanced packet scheduler, which has been studied in [Jia07].

\subsubsection{VoIP packet age aided HSDPA scheduling}

Some VoIP delay sensitive scheduling algorithms in HSDPA (see [Ban05], [Pet07]) were proposed and evaluated previously, in which the power allocation, codes allocation and user multiplexing are scheduled considering the VoIP packet delay requirement as well as the channel conditions. The channel conditions are obtained from the CQI report transmitted on the uplink HS-DPCCH channel. In the prior arts, the packet delay requirement used as the scheduling criteria is based on a static delay budget, e.g., $80 \mathrm{~ms}$, which is derived by simply dividing $150 \mathrm{~ms}$ end-to-end air interface requirement between uplink and downlink components regardless of the practical channel conditions and delays. The static delay budget based scheduling cannot fully take into account the end-to-end user experience. For example, even if the delay is different for the packets transmitted from packet-originated users to the BS due to different HARQ transmission delay, fixed $80 \mathrm{~ms}$ delay budget for downlink scheduling at the BS for the packet-terminated user would be applied without considering the delay difference of the packets. In other words, because of different channel conditions or connection property for a packet-originated user, the delay requirement for each VoIP packet per packet-terminated user under HSDPA scheduling is typically not the same, which can be derived by subtracting the transmission delay occurred at the previous transmissions from total $150 \mathrm{~ms}$ end-to-end delay budget. For example, if it takes $50 \mathrm{~ms}$ for the uplink transmission from the packet-originated user to the BS, then the delay budget for the downlink transmission from the BS to the call-terminated user would be $100 \mathrm{~ms}$, which provides more room for the BS scheduler to improve the overall performance. In another example, suppose the packet-originated user is a fixed-line phone user, the delay requirement for the downlink transmission can be even close to $130 \mathrm{~ms}$, which gives a wide area for performance improvement. Therefore, it does make sense to exploit the realistic delay budget for each packet in order to fully utilize the radio resource efficiently for the higher VoIP capacity while maintaining good end-user experience.

An enhanced HSDPA scheduling algorithm aided by the packet age is proposed in article PIV. The downlink delay budget used for scheduling would be derived from the one way end-to-end delay budget and the "age" of the VoIP packet as expressed in Eq 3:

$$
P D B \underset{u i}{p j}=E 2 E_{u i}^{p j}-\text { PAge }{ }_{u i}^{p j}=250 \mathrm{~ms}-\text { PAge }{ }_{u i}^{p j},
$$

where $P D B \underset{u i}{p j}$ stands for the delay budget used in the downlink scheduling for packet $j$ of user $i, E 2 E_{u i}^{p j}$ the end-to-end delay budget for packet $j$ of user $i$ and PAge ${ }_{u i}^{p i}$ the age of packet $j$ of user $i$. Here, the end-to-end delay budget is the maximum acceptable mouth-to-ear delay. The packet age is defined as the ac- 
cumulative delay from the generation of the VoIP packet at the call-originated user to the instant of the packet arriving at the serving NodeB in the downlink towards the packet-terminated user.

Due to the trend of migrating to the ALL-IP network, the packet age information can be derived from the time-stamp in the IP header. The birth of the packet can be marked by UE in the uplink through the IP layer or by the calling node in the IP network. In IP header, there is an option for the internet timestamp in milliseconds as shown in TABLE 1.

TABLE 1

Timestamp in IP header (see [Ric94])

\begin{tabular}{|c|c|c|c|c|}
\hline 1000100 & Length & Pointer & Oflw & flg \\
\hline \multicolumn{5}{|c|}{ Internet address } \\
\hline & & imestamp & & \\
\hline
\end{tabular}

Thus, for example, the UE packet timestamp information in 3G-GGSN (see FIGURE 8) could firstly be obtained by calculating the accumulative delay according to the time difference between the current time and the packet timestamp. Then, the timestamp information can subsequently be appended for each hop. Finally, the NodeB may derive the age of the packet according to IP header in the case of direct IP connection to NodeB, which is feasible for the case that RNC functions can be integrated into Node-B with the support of IP transportation.

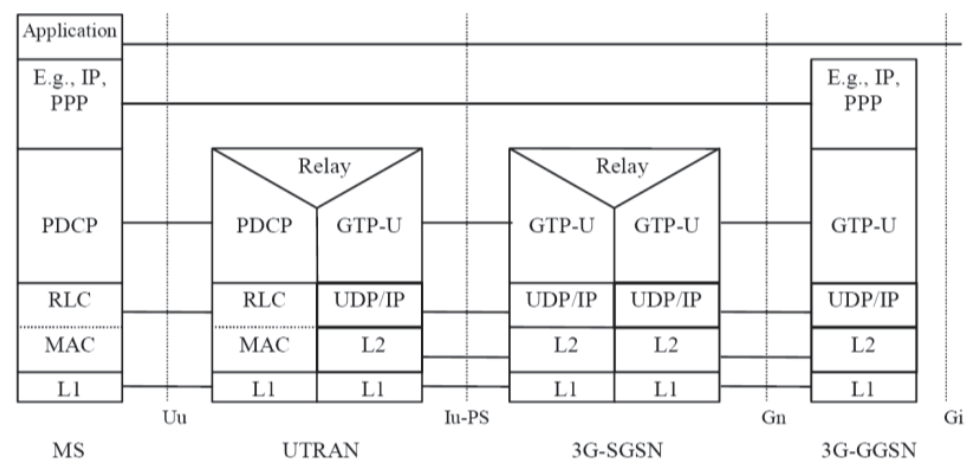

FIGURE 8 3GPP protocol stack (see [Cun03])

However, if RNC and NodeB functions are split and Iub IP transportation is not supported, the age information can be attached into the VoIP packet via the extended field in the frame protocol.

Each VoIP packet can have its own specific delay budget for HSDPA scheduling. The serving NodeB of the packet-terminated users can prioritize packets based on the packet specific delay. In addition, the scheduler can also discard the packet immediately in case the packet age has exceeded the $150 \mathrm{~ms}$ end-to-end delay budget, which gives the opportunity to allocate the radio resources more effectively to improve the VoIP performance. 
The scheduling for VoIP users aided by the packet age is such that, it selects at most $M$ users, which corresponds to the maximum allowed multiplexing user number, from a scheduling candidate set (SCS) by using the Proportional Fair (PF) packet scheduling algorithm which exploits the instantaneous channel state information for each user, and therefore provides a multi-user diversity gain. The selected VoIP users are to be transmitted in the following 2ms Transmission Time Interval (TTI).

The SCS includes VoIP users that fulfill the following criteria:

(i) Users whose packet delay is equal to or larger than a threshold corresponding to the packet specific delay budget.

(ii) Users with pending retransmissions in their hybrid automatic repeat request (HARQ) manager.

If $M$ users are not fulfilled by SCS, other users can be selected according to the priority of PF scheduling.

The novelty of the proposed scheme is two-fold:

- The packet specific delay budget for each VoIP packet will be used instead of the static delay budget by considering the "age" of the packet.

- The age information can be obtained from the optional fields in the IP header supposing IP connection to NodeB in the transmission network. Alternatively without an IP connection to the NodeB, the RNC could inform the NodeB, for example, by appending similar information to each packet via some extended fields in the frame protocol.

With this proposed realistic delay sensitive scheduling HSDPA scheduler for the packet-terminated users can benefit from the knowledge of packet age. For example, for the packets arriving at the buffer earlier than some other packets, they can thus be served with more time for scheduling. On the other hand, BS can prioritize the other packets with a short life time to improve the overall systems performance. Hence, it is more flexible and feasible than the conventional VoIP scheduling based on the static delay budget. It can also de-jitter the buffer delay by scheduling users more efficiently according to the packet specific end-to-end delay budget. Consequently, it can enhance downlink radio transmission efficiency. Besides, the method could be widely used in the other IP-based system such as Long Term Evolution (LTE) system and Wimax for scheduling of real-time traffic.

\subsection{Performance evaluation for VoIP over HSPA}

\subsubsection{Simulation methodology and assumptions}

The quasi-static system level simulators respectively for HSUPA and HSDPA, where all necessary RRM algorithms as well as their interactions are modeled, are used to investigate the performance of VoIP on HSPA. These tools include a 
detailed simulation of the users within multiple cells. The fast fading is explicitly modeled for each user according to the ITU Vehicular-A profile. Regarding to the methodology this type of quasi-static simulator is based on descriptions in [Hol06b]. A wrap-around multi-cell layout modeling several layers of interference is utilized in this study.

The main parameters used in the system simulation are summarized in TABLE 2, where UE moving speeds rather than $3 \mathrm{kmph}$ are mostly applied to investigate the effect of the mobility on the performance of the downlink.

TABLE 2 Simulation parameter settings

\begin{tabular}{|l|l|}
\hline \multicolumn{1}{|c|}{ Parameter System configuration } \\
\hline \multicolumn{1}{|c|}{ Value } \\
\hline Inter site distance & $2.8 \mathrm{~km}$ \\
\hline Cell configuration & ITU Veh-A, Macrocell \\
\hline Voice call mean length & 60 seconds \\
\hline UE speed & $3(30,50,90) \mathrm{kmph}$ \\
\hline Outage observation window length & 10 seconds \\
\hline Cell outage threshold & $5 \%$ \\
\hline Residual FER & $1 \%$ \\
\hline & $2 \%$ PA specific configuration \\
\hline Frame Size & $2 \mathrm{~ms} / 10 \mathrm{~ms}$ \\
\hline Channels & E-DCH/DPCCH \\
\hline Number of HARQ channels & $8(2 \mathrm{~ms} \mathrm{TTI}) / 4(10 \mathrm{~ms} \mathrm{TTI})$ \\
\hline Max number of L1 transmissions & $4(2 \mathrm{~ms}$ TTI $) / 2(10 \mathrm{~ms} \mathrm{TTI})$ \\
\hline Uplink delay budget & $80 \mathrm{~ms}$ \\
\hline Scheduling algorithm & Non-scheduled \\
\hline & $2 \mathrm{~ms}$ \\
\hline Frame Size & HS-DSCH/CPICH/HS-DPCCH \\
\hline Channels & 8 \\
\hline Number of HARQ channels & 4 \\
\hline Max number of L1 transmissions & 4 \\
\hline Max Code-multiplexing Users & 10 \\
\hline Number of code & $20 \mathrm{~W}$ \\
\hline Total BS transmission power & $10 \mathrm{~W}$ \\
\hline HS-DSCH power & $80 \mathrm{~ms}, 150 \mathrm{~ms}$ \\
\hline Downlink delay budget & \\
\hline
\end{tabular}

\subsubsection{VoIP performance in HSPA}

The uplink capacities for VoIP over HSUPA are presented in article PI in which system performance is investigated by the semi-analytical prediction and the system level simulations. The semi-analytical results show the capacity gain of VoIP over HSUPA than $12.2 \mathrm{kbps}$ on DCH in Rel'99. Further, the aforementioned prediction shows the consistency with system level simulation results in principle, which verifies the reliability of the developed system level simulator. From the results, it can be observed that the uplink capacity of VoIP over HSUPA with a non-scheduled algorithm is mainly dependent on the delay budget and availability of the UE power. HARQ and soft handover can be expected to 
lower the required UE power and thus increase the capacity. Due to the time sensitivity of VoIP service, a compromise between the maximum number of HARQ retransmissions and the delay budget can be considered to optimize the capacity of VoIP on HSUPA.

The included articles PI, PII and PIII, investigate the performance of some advanced solutions as described in Section 2.5. For the $2 \mathrm{~ms}$ TTI without uplink packet bundling in the uplink, it can be noted that there can be quite huge power savings by applying DPCCH gating. The exact power saving level would depend on the activity of DPCCH, which is affected by the transmission of EDCH and HS-DPCCH. It also implies that the frequent CQI reporting carried on HS-DPCCH would reduce the power saving gain because of having fewer opportunities to use $\mathrm{DPCCH}$ gating. On the other hand, the performance loss due to less frequent CQI reporting has been evaluated in [Mul05]. Similarly, if the downlink data transmission over HS-DSCH is quite active, the transmission of ACK/NACK feedback information carried over HS-DPCCH in the uplink would be quite frequent, which will also constrain the gating gain.

In particular, TABLE 3 summarizes the VoIP capacity results obtained with a quasi-static system simulator for both $2 \mathrm{~ms}$ and $10 \mathrm{~ms}$ TTI in the case of $3 \mathrm{kmph}$ UE moving speed. The capacity is calculated with two different criteria: the number of VoIP users per sector not exceeding the 5\% FER target measured over $10 \mathrm{~s}$ or the number of VoIP users allowed per sector with an average noise rise of $6 \mathrm{~dB}$. Here the delay budget, i.e. $80 \mathrm{~ms}$, is assumed for HSUPA. This would not be the bottleneck at the performance since HARQ retransmissions will be completed within this delay budget when the max allowed HARQ retransmission number is 1 for $10 \mathrm{~ms}$ TTI and 3 for $2 \mathrm{~ms}$ TTI. The results show that VoIP capacity over HSUPA is comparable or somewhat better than CS voice over DCH (about 65 voice users per sector) with continuous DPCCH and gating gives $40-50 \%$ further gain. Otherwise, fewer transmissions can increase the DPCCH gating gain in the capacity from $50 \%$ to $77 \%$ with $10 \mathrm{~ms}$ TTI because of the increasing opportunities for DPCCH gating from fewer transmissions on E$\mathrm{DCH}$.

TABLE $3 \quad$ Summary of simulation results

\begin{tabular}{|c|c|c|c|c|c|c|c|}
\hline & \multicolumn{2}{|c|}{ Capacity criteria: 5\% FER over 10 s } & \multicolumn{2}{|c|}{ Capacity criteria: Noise rise 6 dB } \\
\hline TTI & $\begin{array}{c}\text { Average num- } \\
\text { ber of trans- } \\
\text { missions }\end{array}$ & $\begin{array}{c}\text { Continuous } \\
\text { DPCCH }\end{array}$ & $\begin{array}{c}\text { Gated } \\
\text { DPCCH }\end{array}$ & $\begin{array}{c}\text { Gating } \\
\text { Gain }\end{array}$ & $\begin{array}{c}\text { Continuous } \\
\text { DPCCH }\end{array}$ & $\begin{array}{c}\text { Gated } \\
\text { DPCCH }\end{array}$ & $\begin{array}{c}\text { Gating } \\
\text { Gain }\end{array}$ \\
\hline $\mathbf{2} \mathbf{~ m s}$ & $\sim 3$ & 82 users & 123 users & $50 \%$ & 75 users & 106 users & $41 \%$ \\
\hline \multirow{2}{*}{$\mathbf{1 0} \mathbf{~ m s}^{*}$} & 1.25 & 65 users & 115 users & $77 \%$ & 61 users & 93 users & $52 \%$ \\
\cline { 2 - 7 } & 1.67 & 80 users & 120 users & $50 \%$ & 73 users & 103 users & $41 \%$ \\
\hline
\end{tabular}

* $10 \mathrm{~ms}$ TTI results using bundling of 2 VoIP frames in a single TTI

In addition, the VoIP performance over HSDPA based on the packet age aided scheduling has been investigated in the included article PIV. The end-toend system performance can be significantly improved by the proposed scheduling which relies on the realistic packet delay requirements derived from the 
timestamp information associated to each packet. The results obtained from extensive system-level uplink and downlink simulations have shown that using the proposed scheduling can lead to an increase in the downlink cell capacity of up to $16 \%$. By applying the proposed method, the downlink performance can be improved considerably while the uplink performance remains the same, which can secure good end-to-end performance.

The included article PV for the book chapter has given a summary for the VoIP performance over HSPA as well as LTE. In general, VoIP performance is promising to achieve the requirements of the operations. It paves the way for the network migrating from CS/PS system towards the ALL-IP based PS system with more efficient operation.

\subsection{Conclusion}

In general, some VoIP gaining techniques (e.g., ROHC, non-scheduled RNC scheduling) make VoIP over cellular system feasible. However, due to the severe obstacles from the control channel overhead and co-channel interference, VoIP performance would be strictly constrained. Thus, some optimized solutions addressing the VoIP bottlenecks in HSUPA cellular systems were proposed and studied. Especially, the control channel overhead reduction solutions adopted by 3GPP are attractive as they can provide good performance. 


\section{D2D ENABLED DATA SOLUTIONS}

It has been envisioned that new innovative local services will make up a significant part of traffic in future cellular systems. In these services, a client and a service provider would often be in the immediate vicinity of each other and much further away from a base station. Therefore, they could potentially have much a better radio channel between themselves than towards a base station. However, the conventional cellular operation cannot handle their mutual traffic any differently but will transfer it via a base station and further route it through the core network. Similar suboptimal relaying of traffic through a base station and the core network occurs in a file share, voice call or other communication between two close-by users of conventional cellular systems. This twofold transmission of the same data, which takes place first in the uplink and then in the downlink, will result in a waste of scarce radio frequency spectrum and a higher end-to-end packet delay in comparison with a direct transmission between the two communicating devices. Meanwhile, it increases the burden of the core network for delivering and processing the duplicated data. Moreover, the possibly longer distances and, therefore, greater path losses of cellular links in comparison with that of a direct link will require much higher transmission power, which in turn results in increased power consumption and intra-system interference.

In the literature, the direct communication between two terminals of an infrastructure-based network has been called various names, out of which we use the device-to-device (D2D) term. Among the first propositions of using D2D communication in conjunction with cellular communication was the one in [Ada98], where this hybrid scheme was considered to be especially suitable for applications such as communication between operational mobile robots and inter-vehicle communication. The latter usage of D2D communication has been an integral part of a number of multi-hop peer-to-peer network extensions proposed for the conventional cellular network topology. This network model was reviewed and its performance for internet access was investigated in [Hsi04]. A survey on D2D communications in various infrastructure-based networks has been carried out in [Leh08]. Network controlled D2D communication without the ad-hoc relaying functionality has also been proposed as an underlay to an IMT/LTE-Advanced cellular network and its performance has been simulated 
in a single-cell scenario and Manhattan grid model with indoor D2D communication [Jan09] and in an indoor environment [Dop09]. In most of the prior studies, D2D concept is aiming for a generic solution without specific consideration on the practical constraints of LTE system. Furthermore, the D2D link distance has been either fixed or artificially limited, and the effect of the D2D user penetration ratio has not been considered. In addition, the channel and system models used have been fairly limited and highly simplified.

Recently, D2D is becoming a hot topic with more and more interest from the academy and industry. In [Vas13] [Asa14] [Alam14], the general concepts and survey on the latest research outcomes have been presented. Service driven D2D communication are discussed in [Lee11] and [Li14] considering the initial service setup procedure and content downloading service. More specifically, the various RRM schemes for D2D communications have also been presented and evaluated. For resource allocation, interference coordination based schemes are extensively studied in [Zhou13], [Xu12], [Wen12], [Han12] and [Wang11]. In addition, game theory is also considered for resource allocation as indicated in [Song14]. For power control schemes, [Gu11] and [Bae13] proposed dynamic power control schemes for interference coordination. In [Wen13], it provides some QoS-aware mode selection and resource allocation schemes. Random access protocol for collision avoidance in cellular device-to-device communication is presented in [Ewa14].

\subsection{Introduction of D2D}

In general, the D2D communication can be deployed either under LTE frequency division duplexing (FDD) cellular system by using the uplink cellular band for D2D communication or under LTE time division duplexing (TDD) cellular system by utilizing the uplink sub-frames of TDD cellular resources. Thanks to the high commonality between LTE FDD and LTE TDD system in the 3GPP system design, it provides the feasibility for such integrated FDD/TDD implementation at UE in terms of cost and complexity. On the other hand, using the downlink resource for D2D communication is not prevented, as indicated in [Chen12].

\subsection{Challenges for D2D}

\subsubsection{Impact of co-channel cross-link Interference}

The considered D2D communication is assumed to use TDD and it takes place in the uplink band of an LTE FDD cellular system. With this setup a dual mode FDD/TDD user equipment (UE) can use the FDD mode for conventional cellular operation and an enhanced TDD mode for D2D communication. The uplink 
was chosen in order to ease out the control of the effects that the D2D communication has on the cellular operation. On the other hand, the interference situation is getting more complicated compared to the conventional cellular system due to the mix of the centralized cellular system and the distributed D2D communication system, which has to be addressed for the success of D2D communication under cellular system. Essentially, there are four types of interference:

- $\mathrm{d} 2 \mathrm{c}$ interference: Interference from D2D transmission to the cellular link reception at eNB.

- $\mathrm{d} 2 \mathrm{~d}$ interference: Cross-link interference between D2D communications.

- c2c interference: Inter-cell interference between cellular transmissions.

- c2d interference: Interference from cellular links to D2D Rx UE.

In addition, the interference can be further categorized as intra-cell and inter-cell interference. There is only c2c inter-cell interference assuming the orthogonal resource allocation for cellular communications in LTE system. However, intra-cell interference can be occurred for $\mathrm{d} 2 \mathrm{c}, \mathrm{d} 2 \mathrm{~d}$, and c2d cases in addition to the inter-cell interference, which depends on resource allocation schemes among D2D and cellular communications. For example, in case of reusing frequency resources, there will be additional intra-cell $\mathrm{d} 2 \mathrm{~d}, \mathrm{~d} 2 \mathrm{c}$ and $\mathrm{c} 2 \mathrm{~d}$ interference, especially for the case with imperfect spatial multiplexing.

The setup and the related interference couplings are further illustrated in FIGURE 9, where it is assumed the D2D users are allocated dedicated resources within a cell while the frequency reuse factor of the cellular network equals one, i.e., there is no intra-cell D2D or cellular interference similarly to the conventional LTE system. Besides, the shared resource allocation for spatial reuse is also allowed to further improve the spectrum usage as long as there is sufficient decoupling among D2D users or between D2D user and cellular users, in which the occurred intra-cell interference from D2D users has to be well controlled to achieve performance gain. 


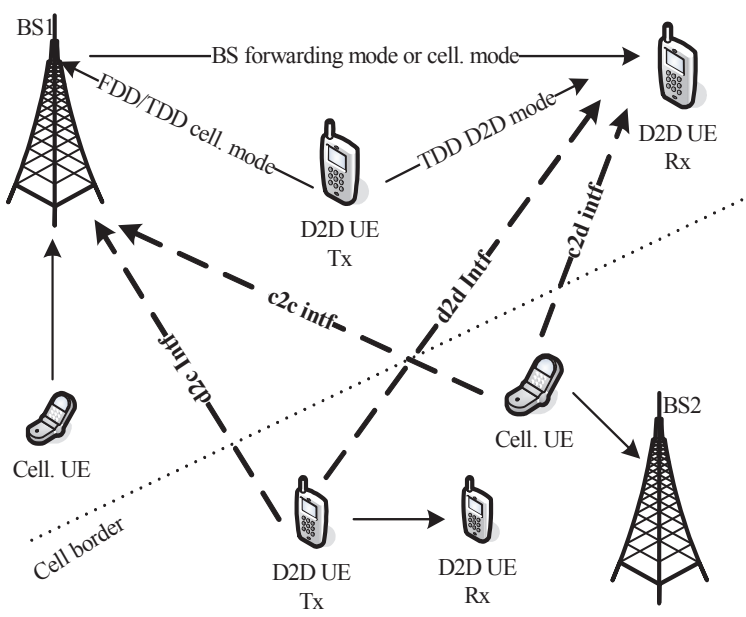

FIGURE 9 D2D and cellular links (solid line) and interference couplings (dashed line) when D2D operation uses the uplink band of a cellular system with the frequency reuse factor of one and dedicated D2D resources within a cell

The Signal-to-Interference-Noise-Ratio (SINR) at a D2D Rx UE per subcarrier for D2D communication can be expressed as Eq 4.

$$
\gamma_{d_{r x}}=\frac{P_{s, d_{r x}} \cdot c_{s, d_{r x}}}{\underbrace{\sum_{j=1, j \neq s}^{N_{t x}} P_{j, d_{r x}} \cdot c_{j, d_{r x}}}_{d 2 d \text { Int } f}+\underbrace{\sum_{k=1}^{N_{c}} P_{k, d_{r x}} \cdot c_{k, d_{r x}}}_{c 2 d \text { Intf }}+\sigma_{d_{r x}}{ }^{2}}
$$

Separately, the perceived SINR at eNB for cellular uplink reception can be represented as Eq 5,

$$
\gamma_{b}=\frac{P_{s, b} \cdot c_{s, b}}{\underbrace{\sum_{j=1}^{N_{t x}} P_{j, b} \cdot c_{j, b}}_{d 2 c \text { Intf }}+\underbrace{\sum_{k=1}^{N_{c}} P_{k, b} \cdot c_{k, b}}_{c 2 c \text { Intf }}+\sigma_{b}^{2}}
$$

where $P_{i, j}$ and $c_{i, j}$ denote the transmit power and the channel gain for the link between device $i$ and $j$ respectively; $s, d_{r x}$ and $b$ stand for the Tx UE, D2D Rx UE and base station; $N_{t x}$ is the number of D2D Tx UEs and $N_{c}$ is the number of cellular UEs; ${\sigma_{d_{r x}}}^{2}$ and ${\sigma_{b}}^{2}$ represent additive white Gaussian noise at D2D Rx $\mathrm{UE}$ receiver and base station receiver separately.

According to the SINR Eq 4 and Eq 5, it can be noted that the overall system performance in a mixed system with both D2D and cellular communications can be affected by several factors: D2D pathloss controlled by D2D mode selection criteria, D2D transmit power control scheme, frequency resource allocation strategy and the proportion of D2D communication. These factors are also tightly coupled between each other, which makes a big challenge for system operation and optimization.

Besides, it is interesting to notice that full duplex TDD is also under consideration recently for D2D implementation as presented in [San14]. When full 
duplex communication is adopted for D2D communication, it requires only one frequency band for two way communication between the local users. It can further improve the bandwidth efficiency for D2D communication. However, more challenges and restrictions have to be addressed.

\subsubsection{Impact of near-far interferer on ADC range}

The Analogue-to-Digital Converter (ADC) and Automatic Gain Control (AGC) typically work together to center the dynamical range of the ADC around the stronger signal at the UE receiver, which could be the intended transmitting (Tx) signal or a near-far Tx interfering signal. AGC is be used to adjust the gain of the variable gain amplifier (VGA) to its optimal output level (see [Ale11]). In principle, AGC would detect the peak signal level via the peak detector, and compare it to a reference voltage, finally filter and generate the required control voltage for a stable output. Especially, the feedback loop based AGC used widely can lower the input dynamic range and reach the higher linearity. However, it may suffer from instability with high compression or explanation, and require the faster AGC settling-time. Thus, the resolution of an ADC is important for mitigating the trouble caused by high compression. Typically, the resolution of ADC, denoted by $Q$, is $Q=[-V ;+V] / N$, where $V$ is the maximum magnitude and $N$ the number of quantization levels. Considering an example with a 12-bit ADC converter (4096 levels), if the received power from the near-far interferer is $27 \mathrm{~dB}$ (500 times) higher than the wanted signal, the wanted signal magnitude would be represented by about 4 ADC quantization levels $(V / 500 \sim=4$ * $2 \mathrm{~V} / 4096)$, which results in severe truncation. In practical ADC, the wanted signal may be removed entirely by ADC as some headroom is used as a fading margin to avoid signal truncation due to positive fade. On the other hand, it should be noticed that the high resolution of ADC may lead to higher implementation costs.

For TDD based D2D links transmitting and receiving on the same frequency band as LTE cellular users' transmission in the hybrid network, this kind of desired signal truncation suffers from the near-far interference is becoming more severe than the pure cellular network. Usually, the LTE cellular users will transmit with the power level according to fractional power control (FPC) specified in [4G213] that compensates path loss between them and the base station but does not limit the near-far interference to $\mathrm{D} 2 \mathrm{D}$ receivers, which will cause the near-far interference to D2D users in the case of frequency resource reusing. In short, the near-far problem would cause the receiver to capture a strong interference signal but not receive a weaker wanted signal. Especially, the cellular UEs in the cell edge typically transmits at high power are likely to create strong interference to near-by D2D UEs in receiver mode. Besides, the imperfect spatial reuse lacking of the real-time neighbor information may also lead to strong co-channel interference among D2D users and even block the desired signals due to the signal truncation in ADC/AGC operation. Otherwise, it is difficult to apply an efficient power control scheme as performed in the well planned and deployed cellular networks to ensure that all 
parallel transmitting signals arrive at the D2D receiver with a similar power level, because the deployment of the intended D2D receivers is not under the control of the operators, and also difficult to predict owing to the UE mobility and dynamics of a user behavior.

\subsubsection{Impact of inter-carrier interference (ICI)}

Even in the case of frequency resource sharing between cellular users and D2D users, the inband emissions in UE transmissions due to imperfection of the radio frequency (RF) transceiver such as frequency offset and phase noise would destroy the orthogonality of the victim UE signal, consequently causing the inter-carrier interference (ICI), which has been investigated in [Bas08]. The minimum requirements for inband emission have also been specified in [4G101] for the LTE system to ensure that there is no significant performance degradation in the eNB receiver. The in-band emission is defined as the average across 12 subcarriers and as a function of the resource block (RB) offset (i.e., delta RB) from the edge of the allocated UL transmission bandwidth. It is measured as the relative UE output power of any non-allocated $\mathrm{RB}(\mathrm{s})$ and the total UE output power of all the allocated $\mathrm{RB}(\mathrm{s})$.

FIGURE 10 shows that depending on the allowed error vector magnitude (EVM) level of the selected modulation scheme, QPSK or 16QAM, the maximum allowed inband emissions can be around 18 or $21 \mathrm{~dB}$ for the small RB offset respectively, and leveling off around $25 \mathrm{~dB}$ for the larger RB offset.

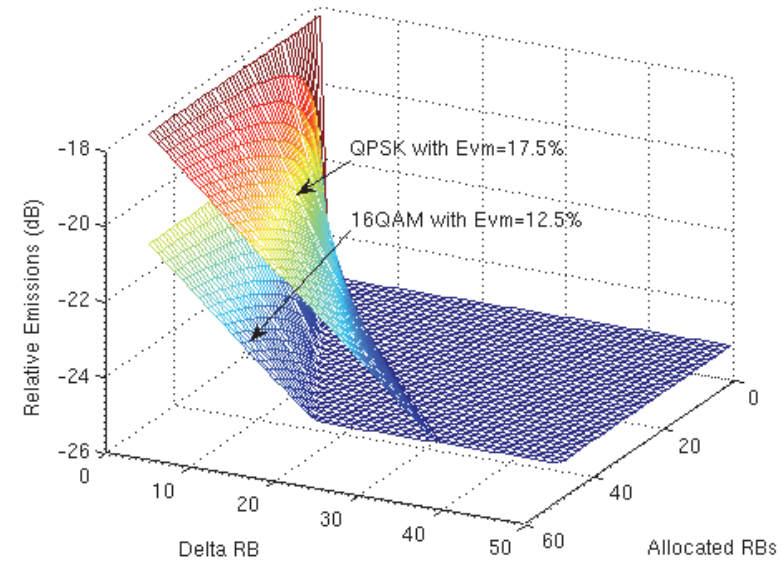

FIGURE 10 Illustration of inband emissions

Consequently, the near-far effect in D2D integrated LTE cellular network, where one interfering UE close to a D2D Rx UE may transmit at a much higher power than the D2D Tx UE, can aggravate the impact of ICI in the D2D UE receiver. Otherwise, it hints that the frequency orthogonality may not be ideal due to the imperfection of RF transceiver in the device, and accordingly some 
typical solutions such as frequency hopping used in pure cellular network may not be suitable for the resource allocation in the hybrid network.

\subsubsection{Scheduling fairness with mixed D2D and cellular operations}

Given a fair and efficient split of resources between users, the so-called 'proportional fair' (PF) packet scheduler is typically adopted in the conventional cellular system. The PF scheduler leverages the fairness and achievable throughput while improving coverage performance. The principle of the PF scheduler is to schedule the users on top of their fades, as illustrated in FIGURE 11. In other words, the user is scheduled with the best relative channel condition among the candidate users. To be noticed, PF scheduling is regardless of the absolute channel conditions.

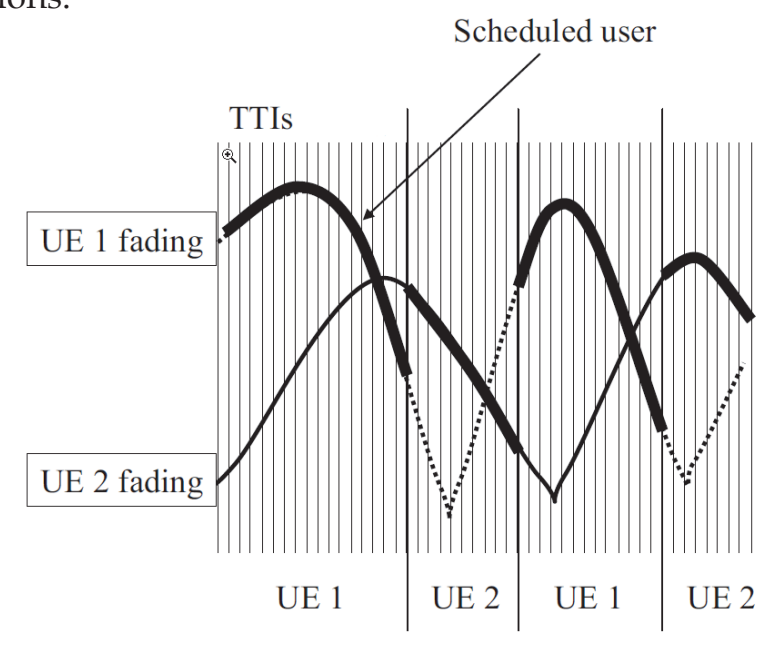

FIGURE 11 Principle of PF scheduler [Hol11]

For example, as descripted in [Jal00] the PF scheduler will select the user $j$ with best relative channel quality:

$$
j=\arg \max _{i}\left\{\frac{\gamma_{i}(t)}{\overline{\gamma_{l}}}\right\}
$$

Here, $\gamma_{i}(t)$ is the instantaneous channel condition experienced by user $i$, which can be SINR or the predicted throughput derived from the reported CQI value. $\overline{\gamma_{l}}$ is the average channel condition for user $i$. This rule ranks the users according to the ratio of their instantaneous channel quality over their own average channel conditions. Given the relative constant interference levels for all users in the conventional cellular downlink operation with the similar downlink transmission power from eNB, the multiuser diversity can be exploited while the issue of fairness among the users is addressed effectively.

However, in the hybrid cellular system with both D2D based ad-hoc and cellular communications, the centralized PF scheduler may favor D2D users more than the cellular users due to a larger dynamic range of PF metric for D2D 
users which is mainly caused by a dramatic change of the interference level at D2D users. With the complicated interference environment in the hybrid network as discussed above and different transmission power per D2D user, it is likely that PF metric of D2D is much higher or lower than the one for cellular users. However, as long as there are sufficient D2D users with i.i.d CQI reports, the scheduler would likely pick up a D2D user for scheduling due to the higher PF metric than most cellular users. In other words, a single PF scheduling priority queue cannot apply for this case because of improper comparison of the PF metric between the cellular and D2D users.

For example, as shown in FIGURE 12 from the system level simulation results for the case with both LTE cellular and D2D users, the scheduling activities based on the PF algorithm are clearly presented with two stripes, of which the upper is for D2D UEs and the lower for Cellular UEs. It can be noted that D2D users with potentially higher PF metrics would be overscheduled in comparison with cellular users. This is because the D2D users may experience occasionally much more severe interference from the neighbor cellular and D2D transmissions, which severely decreases their average SINR.

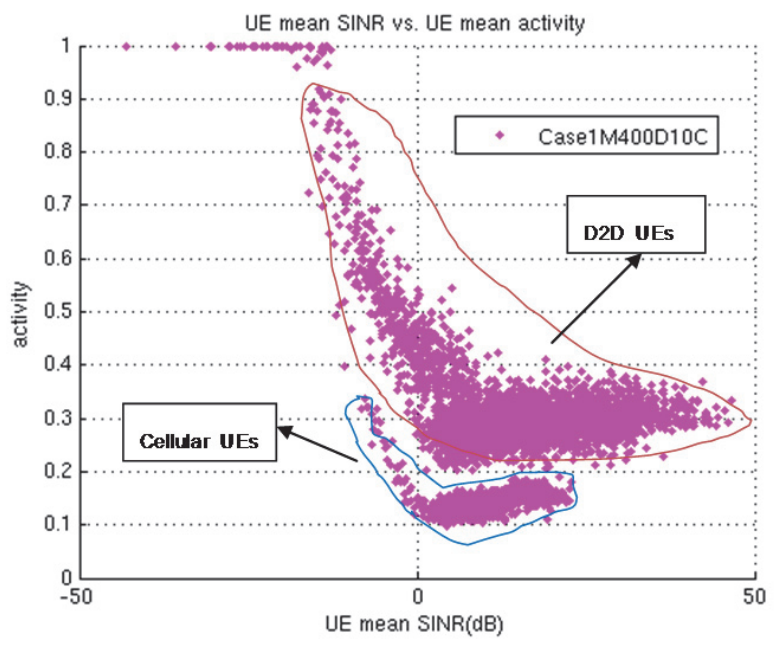

FIGURE 12 UE scheduling activity vs. UE average performance (average SINR); Case 1 $(\mathrm{ISD}=500 \mathrm{~m}), 25 \mathrm{UEs} /$ Cell (10 D2D UEs and 15 Cellular UEs)

In general, the scheduling to address both cellular and D2D users fairly should be taken into account in the system design. In particular, it may affect CQI reporting mechanism of D2D users. 


\subsection{RRM for D2D}

\subsubsection{Mode selection}

A pair of close-by cellular users supporting D2D operation can communicate by using the conventional cellular mode, the simplified cellular mode or D2D mode of operation. The simplified cellular mode is an optimization of the conventional cellular mode based on packet direct forwarding or routing via eNB rather than the gateway, which can significantly reduce the end-to-end packet delivery latency. In short, latency can be expressed below for the conventional cellular mode $\left(T_{c c}\right)$, the simplified cellular mode $\left(T_{s c}\right)$ and the direct D2D cellular mode $\left(T_{d 2 d}\right)$ :

$$
\begin{array}{cc}
T_{c c}=t_{u l}+t_{c n}+t_{d l} & \text { Eq } 7 \\
T_{s c}=t_{u l}+t_{d l} & \text { Eq } 8 \\
T_{d 2 d} \approx t_{u l} & \text { Eq } 9
\end{array}
$$

where $t_{u l}$ and $t_{d l}$ denote the delay for the successful packet delivery in uplink and downlink air interface separately including the Hybrid automatic repeat request (HARQ) (re)transmission delay and UE/eNB processing delay; $t_{c n}$ stands for the routing delay for the packet in the core network. It is clear that the simplified cellular mode based on packet routing or direct forwarding via layer 2 function can avoid the huge latency in the core network caused by the conventional cellular mode using layer 3 routing (up to 200ms delay reduction) whereas the direct D2D transmission mode can further reduce the half latency thanks to one-hop instead of two-hop transmission in the simplified cellular mode. Considering the fact that the devices with the potential for D2D communication are typically close to each other and under the same eNB, the simplified cellular mode can be exploited as one mode to offer the D2D service.

To maximize the system throughput, the optimal criterion for selecting between these two modes would be the sum-rate criterion. That is, the mode which would result in the highest system sum-rate would get selected. For optimality, the mode selection should also be done jointly with power control and resource allocation based on instantaneous channel and interference conditions. This, however, would largely increase complexity and control signaling. Depending on the phase of D2D operation, Initial D2D link setup phase or D2D operation maintenance phase, the mode selection criteria can be different considering the tradeoff between the performance and link management complexity.

In the initial D2D link setup phase, mode selection can be based on a longterm measurement, e.g., pathloss or the received power. The purpose is to establish a stable communication link for initial D2D setup. During this phase, eNB lacks the full knowledge of link quality due to the limitation on channel information reporting. In this case, we resort to a suboptimal but much simpler 
and more practical approach where the mode selection is done based on the received power level.

The D2D mode is selected if the smaller of the powers received at the two directions of a D2D link between two users would be higher than the smaller of the two powers that a base station would receive from these two users. Otherwise the users operate in the cellular mode. The above criterion can be also expressed as Eq 10:

$$
\min \left(P_{1,2, d_{r x}} \cdot c_{1,2}, P_{2,1} \cdot c_{1,2}\right)>\min \left(P_{1, b} \cdot c_{1, b}, P_{2, b} \cdot c_{2, b}\right) \quad \text { Eq } 10
$$

where $P_{i, j} \cdot c_{i, j}$ is the power from unit $j$ that is received at unit $i$ and unit index $b$ belongs to the base station and indices one and two to the D2D users. With this criterion we assume that the bidirectional cellular link between the users is limited by the uplink and that the users may have different transmission powers also in D2D operation. Note that if the transmit powers of the links considered in Eq 10 are the same, this criterion is reduced to selecting the mode with the lowest path loss.

Although the above simple suboptimal mode selection criterion ensures that the mode and link with the highest signal level is chosen, it does not take into account the interference level at the receiver. In particular, the presence of strong inter-cell interference variation during the operation at the cell edge may eventually lead to degraded performance in the D2D mode. However, during the D2D operation phase with the established D2D link and the simplified cellular link, eNB can make a fast or dynamic mode selection based on the frequent channel quality indication (CQI) report which can capture the effect of varying interference. In this case, the criteria can be established based on the short-term D2D SINRs $\left(\gamma_{2,1}\right.$ and $\left.\gamma_{1,2}\right)$ derived from the reported D2D CQIs, and cellular uplink SINRs $\left(\gamma_{1, b}\right.$ and $\left.\gamma_{2, b}\right)$ estimated from the uplink sounding reference signal (SRS). Similarly to Eq 10, the SINR based criteria can be expressed as Eq 11.

$$
\min \left(\gamma_{2,1}, \gamma_{1,2}\right)>\min \left(\gamma_{1, b}, \gamma_{2, b}\right)
$$

In practical operation, dynamic mode switching would require the unique sequence number for the packet by D2D Tx UE so that the D2D Rx UE can reorder the packets received from either eNB forwarding or D2D direct transmission, as indicated in FIGURE 9. Dynamic mode switching can be transparent to the D2D UEs due to the characteristics of broadcasting transmission. Whether to dynamically switch is fully controlled by eNB via the resource allocation grant.

One use case for dynamic mode switching is illustrated in FIGURE 13. In this case, based on the established D2D communication eNB can derive the SINRs for two modes based on D2D CQI reports and SRS estimation. D2D Tx UE will transmit the data to Rx UE using the resources indicated by the D2D resource grant from eNB. Meanwhile, the packets can also be received by eNB, given the suitable transmit power setting. In the retransmission phase triggered by D2D negative-acknowledge (NACK), eNB can choose whether to retransmit the data by eNB forwarding mode or D2D direct mode supposing the packet 
has been received by eNB successfully in the initial transmission. In case of eNB forwarding mode, eNB will suspend the resource allocation to D2D Tx UE for retransmission. Instead, eNB would retransmit the data directly to D2D Rx UE. For each retransmission, eNB can dynamically determine the mode of communication based on the estimated SINRs. eNB can resume the D2D transmission via a D2D resource grant for either new transmission or retransmission. The dynamic mode switching can secure the robust and flexible communication between two devices, especially in case of varying channel and interference conditions. In addition, it can also reduce the retransmission delay since there is no need of D2D grant with UE processing (up to 3ms delay) compared to eNB direct forwarding mode.

Besides, it should be kept in mind that there is another benefit of downlink resource saving with direct D2D communication only using the uplink resources, compared to the simplified or conventional cellular transmission mode where BS has to transmit the same data to the peer user using the downlink resources. Essentially, this also provides the potential for D2D communication triggered by offloading the traffic in cellular downlink for the scarce downlink resources.

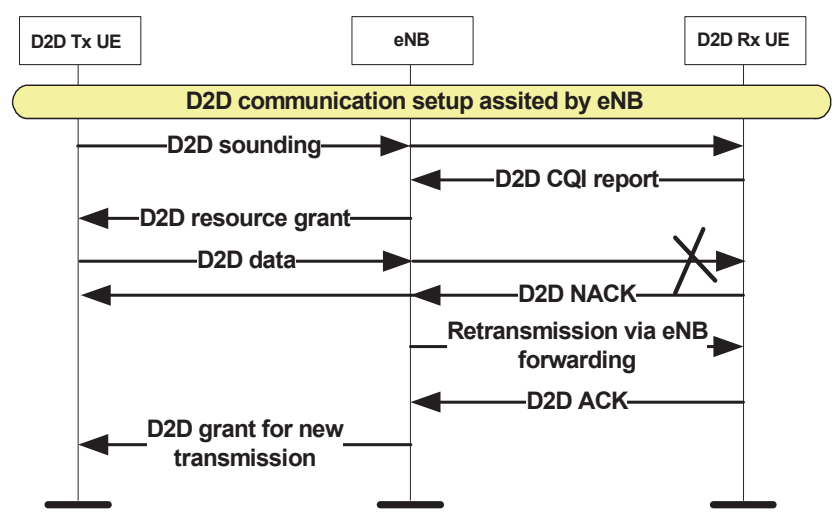

FIGURE 13 Sequence diagram for dynamic mode switching

Thus, there can be different criteria for the mode selection depending on the target of network optimization. In general, the interference caused to and received from the other users in the D2D mode can be affected to some extent by a power control and resource allocation scheme, which will be further discussed in the following sections. For simplicity, the term "cellular mode" would be used to represent the simplified cellular mode below.

More details have been discussed in article PVI.

\subsubsection{Power control}

Both the cellular and D2D links use the fractional power control according to the LTE specifications [4G213]. For the D2D links, we consider two schemes which differ in what path loss is to be fractionally compensated. The first 
scheme targets the path loss in the D2D link, whereas the second scheme uses the loss of the cellular link towards the serving base station. In principle, the transmit power for D2D communication in subframe $i$ can be determined by Eq 12:

$$
P_{d 2 d}(i)=\min \left(P_{c}(i), P_{0}+\alpha \cdot P L+10 \cdot \log _{10}(M(i)) \quad \text { Eq } 12\right.
$$

where $P L$ is the pathloss for either cellular link or D2D link depending on the PC scheme to be discussed later, $P_{c}(i)$ is the transmit power assuming the cellular mode operation in subframe $i$, which is derived based on the cellular link pathloss for $P L$ in Eq 5 , and $P_{0}$ is the power per PRB, $M(i)$ is the allocated bandwidth in subframe $i$ for data transmission measured in the number of physical resource blocks (PRBs) specified in [4G213], ], $\alpha$ is for partial pathloss compensation with a value in between 0 and 1 . Specifically, supposing $\alpha=1, P_{0}$ can be interpreted as the target received power per PRB to achieve a certain SNR.

Essentially, the D2D link based power control of D2D users operates on the same principle as the power control of cellular users, but due to the shorter average link distances of D2D users with the proper mode selection, their transmit power levels are much lower. This saves D2D users' battery resources and decreases their interference towards other users in comparison to their operation in the cellular mode. On the other hand, the low D2D link power without consideration of the interference may be overshadowed by nearby cellular users, especially in the cell edge where the transmission powers of the neighbor cellular users are at the highest. This disadvantage can be countered by the cellular link based power control that allows the user to transmit in the D2D link with the same power as it would if it was transmitting to the base station. This power also serves as the upper limit for the D2D link based power control, which implies that D2D transmission won't cause more interference to the incumbent cellular users than the conventional cellular mode. With the cellular link based power control option, however, D2D transmission does generate more interference in the system, but not more as it would in the cellular mode. Thus, a sensible D2D PC scheme can effectively reduce the power consumption and/or enhance the overall system performance, as investigated in article PVI.

\subsubsection{Resource allocation}

D2D operation under the cellular network with the centralized control system provides the opportunity for operators to effectively manage the interference between D2D and cellular communications while offering the compelling service with high spectrum efficiency. In general, there are several ways for radio resource allocation, depending on the level of coupling between D2D communication and the cellular network for radio resource allocation.

Intuitively, one of the radio resource allocation schemes for 2D links can be similar to that of conventional cellular users (aka, network fully controlled D2D). That is, D2D pairs are scheduled dedicated resources by their serving 
base station in the same way as the base station schedules its cellular users. Moreover, the D2D links have access to all the same time and frequency resources as the cellular links. Both link types are scheduled by using the proportional fair scheduling algorithm and are assigned the same number of PRBs. In this case, eNB is fully in charge of D2D communication including mode selection, connection establishment, radio resource allocation, etc. So eNB can exploit the centralized information from both communication modes to optimally allocate the resources for maximizing the systems overall performance.

Although the abovementioned centralized resource allocation scheme can avoid intra-cell interference, there have been several disadvantages. Firstly, the signaling cost for exchange of D2D link status information between the devices and eNB is prohibitively high. For example, supposing $n$ pairs of D2D devices, the D2D link status reports from $2 n$ D2D devices will be sent to eNB in addition to the cellular link reports. Moreover, considering the uncertainty of the link quality due to the interference from the other D2D device transmissions, $\frac{n !}{(n-2) !}$ D2D link status reports are required in the worst case to include the interference status from all the potential interfering D2D links. Even though not all neighbor D2D device transmissions will cause the interference, the number of the required reports with a few interferes is still expected to be high. Also the D2D interferers may be changed dynamically due to UE mobility, which will require frequent reporting causing a high cost in signaling. In addition, the potential interferers from the cellular UEs have to be considered as well, which also requires the link status reports. Further, when the cell-edge devices for D2D communication belong to the different eNBs, signaling for information exchange between eNBs with independent scheduling are needed to effectively avoid the $\mathrm{d} 2 \mathrm{~d}$ co-channel interference as discussed in Section 3.2.1, which will inevitably increase the complexity, signaling overhead, and scheduling delay in the network. Eventually it may degrade the systems overall performance.

While the abovementioned centralized resource allocation scheme with dedicated resources can avoid the intra-cell interference, it is challenging to employ the spatial reuse from the distributed D2D communication for the higher spectrum efficiency due to the signaling cost and the dynamics of D2D interferers. Instead, a decentralized resource allocation scheme is considered for D2D resource allocation by means of autonomous scheduling for D2D pairs. The D2D pairs use the sub-band frequency resources with the highest scheduling metric (e.g., the best channel status) in the frequency domain. This may result in a blind reuse of resources scheduled for either cellular or other D2D users in the cell. The spatial reuse of the resources within a cell can increase the spectral efficiency if the caused intra-cell interference can be tolerated, avoided, or suppressed. In case of sparse deployment of D2D devices, the co-channel interference due to spatial reuse between D2D communications is expected to be acceptable due to the large path loss with less coupling. However, in case of dense deployment with tight interference coupling, there could be a risk of over-reusing the resources with the high co-channel interference, because the estimated channel status for resource selection may not fully take into account 
the transmission status (Tx On/Off) of the D2D/cellular interferes and the situation of simultaneous reusing the same resource by the other D2D/cellular devices. In this case, the caused high intra-cell interference eats up the gain from the spatial reuse. However, it should be noted that the available radio resources are increased for both the cellular and D2D users in comparison with the centralized scheme allocating dedicated resources for D2D and cellular links. This provides the potential for a higher frequency diversity gain, which becomes beneficial especially in the less loaded network where there is less collision on the resource allocation. One more benefit for the autonomous reuse is the low signaling overhead in the practical operation. However, in case of a high load, the gain of spatial reuse may rely on a more precise interference control to decouple links sharing the same resources. In this case, the network controlled reuse scheme for resource allocation associated with mode selection and power control may be more favored.

Instead of the centralized and decentralized D2D operation which have been evaluated in article PVI, semi-autonomous D2D can be considered as another option for D2D operation under the cellular network. Essentially, it uses the RTS/CTS mechanism with the assistance signaling provided by the eNB to avoid both c2d and $\mathrm{d} 2 \mathrm{~d}$ co-channel interference. The fundaments of the semiautonomous D2D are described in Section 3.4 and more details can be found in article PVIII.

\subsubsection{Time hopping for co-channel interference mitigation}

Time Hopping (TH) of D2D resources within the cell and across cells is carried out to randomize near-far interference from nearby transmitting D2D and cellular UEs, which has been investigated in article PVII. This kind of interference could be from co-channel interference and/or ICI. The base station (i.e., eNB in LTE) applies random sequence offsets at regular time intervals to semipersistent scheduled resources, accordingly preventing one pair from excessively suffering eNB-unaware near-far interference by another pair for a longer duration. The random sequence offset used in time hopping of resources allocated to device pairs/clusters may be:

- Based on device pair/cluster location.

- In the short term for D2D devices within the same cell and spatially separated from other D2D devices in neighbor cells.

- In the long term via eNB co-ordination over LTE X2 interfaces with a latency of at least $20 \mathrm{~ms}$.

The principle of $\mathrm{TH}$ is to randomize the overall channel access collisions and minimize the occurrence of a collision between victim UEs and the strongest interfering UEs.

eNB can use Semi-Persistent Scheduler (SPS) as specified in [4G321] to schedule UL grant resources to a cluster head and slaves using with periodicity of the UL grant every $x$ subframes $(x=10,20, . ., 120$ as specified in [4G331] for 
$s f x)$. The clusters are formed by the eNB based on max D2D transmitting Power of UEs and geographical location estimation of devices (e.g., Timing Advance parameters and Direction of Arrival). UL grant resources allocated to a cluster aim to minimize inter-cluster interference by spatial orthogonality and/or time orthogonality (subframe separated), and if neither spatial nor time orthogonality is feasible, frequency orthogonality is applied via separated RBs. Note that frequency orthogonality cannot in itself deal effectively with near-far interference typically experienced in D2D communication due to inband emissions of adjacent RBs from other UE Tx, which has been demonstrated in FIGURE 10.

For example, the devices pairs P1, P2, P3, P4, and P5 in FIGURE 14 are allocated with TH sequence $S 1, S 2, S 3, S 4$, and $S 5$ respectively. A simple random $\mathrm{TH}$ offset for device pair resources within a same geographical area (i.e. not spatially separated) is assumed. Let us denote the spatial groups including device pairs $\{P 1, P 4\}$ by $G 1$, and $\{P 2, P 3\}$ by $G 2$.

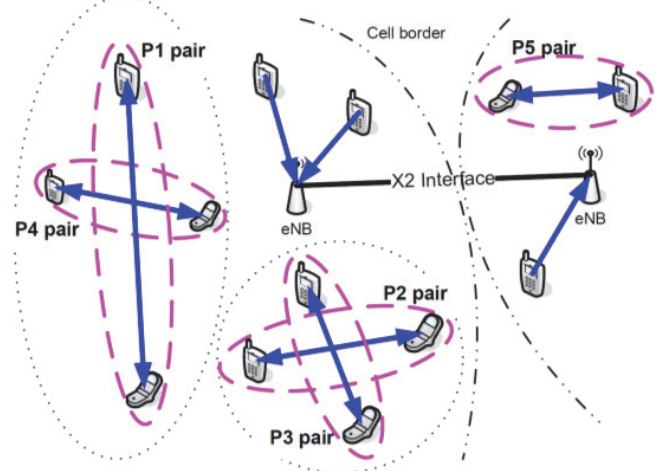

FIGURE 14 Illustration of resource allocation

The time hopping procedure can be expressed as two steps:

Step 1: Divide the radio resources as several orthogonal groups in the time domain, in which each group has a pre-defined periodical transmission timeslots as below:

$$
\begin{aligned}
& t_{j, 1}=N G^{*}\{1,2,3, \ldots, N P\}=N G, 2^{*} N G, 3^{*} N G, \ldots, N G^{*} N P ; \\
& t_{j, 2}=t_{j, 1}-1=N G-1,2^{*} N G-1, \ldots, N G^{*} N P-1 ; \\
& \ldots \\
& t_{j, i}=t_{j, i-1}-1=N G-i+1,2^{*} N G-i+1, \ldots, N G * N P-i+1 ; \\
& \ldots \\
& t_{j, N G}=t_{j, N G-1}-1=1, N G+1, \ldots, N G *(N P-1)+1 ;
\end{aligned}
$$

Where NG denotes the number of TH resource groups, NP for the number of $\mathrm{TH}$ period, and $\mathrm{j}$ for the $\mathrm{TH}$ timeslot index expressed as a multiple on $\mathrm{NP}$ timeslots.

Step 2: D2D pairs are allocated to different resource groups in a period of $N P$ via eNB SPS scheduler or the (random) selection by the D2D pairs themselves.

For example, $\mathrm{NG}=3$ and $\mathrm{NP}=3$ may give timeslot index sequences $t_{1,1}=\{3$, $6,9\}, t_{1,2}=\{2,5,8\}, t_{1,3}=\{1,4,7\}$, which in turn allocates timeslots $\{s 3, s 6, s 9\},\{s 2, s 5$, 
$s 8\}$, and $\{s 1, s 4, s 7\}$ to devices $P 2, P 3$, and $P 5$ in first term of sequence $S 2, S 3$, and $S 5$ respectively. Further, as shown in the example, the timeslot index sequences are allocated with $N G^{*} N P=9$ timeslot periodicity. Hence, we assign $t_{j, 1}, t_{j, 2}$, and $t_{j, 3}$ to device pairs $P 1, P 2, P 3, P 4$, and $P 5$ with a random TH offset to define TH sequences $S 1, S 2, S 3, S 4$, and $S 5$ respectively as follows

$$
\begin{aligned}
& S 1=\left\{t_{1,2}, t_{2,3} \ldots\right\}=\{s 2, s 5, s 8, s 10, s 13, s 16, \ldots\} \\
& S 4=\left\{t_{1,1}, t_{2,2} \ldots\right\}=\{s 3, s 6, s 9, s 11, s 14, s 17, \ldots\} \\
& S 2=\left\{t_{1,3}, t_{2,1} \ldots\right\}=\{s 1, s 4, s 7, s 12, s 15, s 18, \ldots\} \\
& S 3=\left\{t_{1,1}, t_{2,3} \ldots\right\}=\{s 3, s 6, s 9, s 10, s 13, s 16, \ldots\} \\
& S 5=\left\{t_{1,2}, t_{2,2} \ldots\right\}=\{s 2, s 5, s 8, s 11, s 14, s 17, \ldots\}
\end{aligned}
$$

Note that the same TH timeslot index sequence, $t_{j, i}$, may not be used by more than one device pair within the same spatial group to prevent intra nearfar interference. Compare this set of sequences using random $\mathrm{TH}$ offset to the following sequence set without such $\mathrm{TH}$.

$$
\begin{aligned}
& S 1^{\prime}=\left\{t_{1,1}, t_{2,1} \ldots\right\}=\{s 3, s 6, s 9, s 12, s 15, s 18, \ldots\} \\
& S 4^{\prime}=\left\{t_{1,2}, t_{2,2} \ldots\right\}=\{s 2, s 5, s 8, s 11, s 14, s 17, \ldots\} \\
& S 2^{\prime}=\left\{t_{1,2}, t_{2,2} \ldots\right\}=\{s 2, s 5, s 8, s 11, s 14, s 17, \ldots\} \\
& S 3^{\prime}=\left\{t_{1,1}, t_{2,1} \ldots\right\}=\{s 3, s 6, s 9, s 12, s 15, s 18, \ldots\} \\
& S 5^{\prime}=\left\{t_{1,3}, t_{2,3} \ldots\right\}=\{s 1, s 4, s 7, s 10, s 13, s 16, \ldots\} \\
& S 1^{\prime}, S 2^{\prime}, S 3^{\prime}, S 4^{\prime} \text {, and } S 5^{\prime} \text { can readily be scheduled by SPS. Note that the }
\end{aligned}
$$
SPS allocates UL grants every $x$ subframes. Hence, device pairs P1, P2, P3, P4, and $P 5$ are allocated UL grants by SPS every $x=9$ subframes. Further, consider the case where transmitting devices in device pairs P4 in spatial group G1 may cause strong interference to a receiving device in device pairs $P 2$ in spatial group G2. This could be the case when there is no effective spatial orthogonality between $P 4$ and $P 2$, perhaps due to devices in $P 4$ being in an elevated position or having a direct line of sight (LOS) to devices in P2. In the example, without $\mathrm{TH}$, device pair $\mathrm{P} 4$ are allocated UL grants by SPS in the same time slots as device pair $P 2$ and will cause interference in all the timeslots.

The near-far interference with and without TH for this example is illustrated in FIGURE 15. So, with TH, only a few time slots allocated to device pair $P 2$ will suffer such interference from device pair $P 4$. 


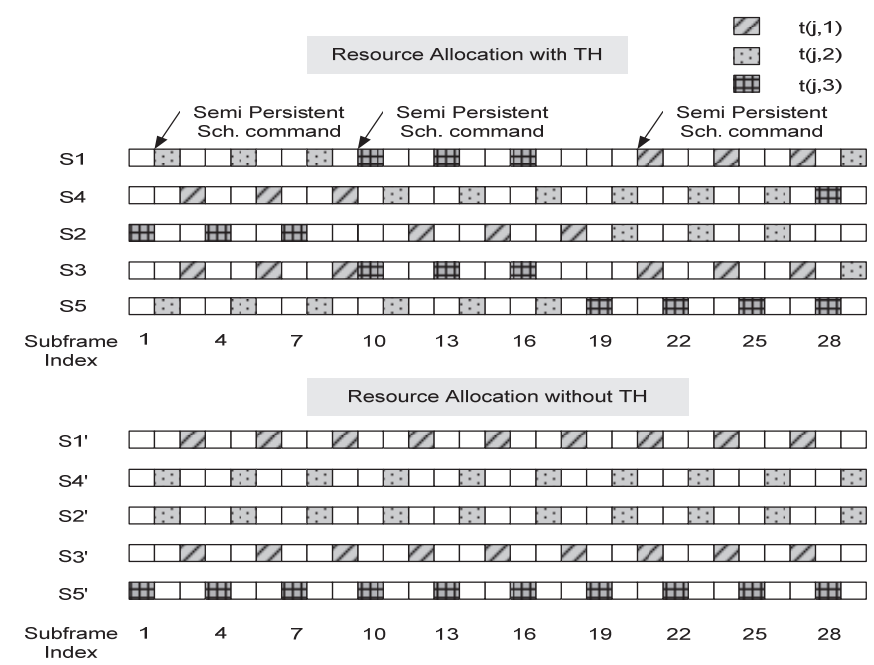

FIGURE 15 Illustration of resource allocation w/o TH

Comparing to the TH with eNB SPS scheduler, the event triggered TH and random TH by D2D pairs can be performed with less signaling overhead.

Recently, the further extension for this concept has been performed in [Ye14] with more analytical results to explore the impact of key parameters on the networks performance. Moreover, using a genetic algorithm with frequency hopping in device to device communication for interference mitigation was recently proposed in [Lee12] to explore the hopping gain in the frequency domain.

\subsubsection{Enhanced scheduler with optimized D2D CQI reporting}

As discussed in Section 3.2.4, there is a challenge for the centralized resource scheduling to offer fair scheduling among D2D and cellular users while keeping performance. In [Chen14], an enhanced scheduler with optimized D2D CQI reporting is proposed to address this issue. The principle of the idea is to prioritize scheduling of D2D users with an active D2D CQI report which is triggered by a scheduling relevant event.

The procedure of the operation has been presented in FIGURE 17, which comprises the following key steps:

- eNB calculates D2D scheduling activity factor (“ $\left.\alpha^{\prime}\right)$ according to the proportion of D2 users and cellular users to be scheduled. The scheduling activity factor indicates the resource partition between D2D users and cellular users.

- For example, supposing 3 D2D pairs and 7 cellular users served by eNB, $\alpha=3 /(7+3)=30 \%$, which implies $30 \%$ resources (time and/or frequency) would be allocated to D2D users for fair scheduling among users. 
- eNB would send " $\alpha$ " value to D2D users for configuration of an event triggered D2D CQI reporting

- Upon receiving " $\alpha$ " value from eNB, a D2D user would determine a threshold for event triggered reporting based on " $\alpha$ " value and the build-in historical SINR distribution curve. As illustrated in FIGURE 16, a SINR value corresponding to $70 \%$ (i.e., $1-\alpha$ ) of SINR CDF curve would be selected as a threshold to trigger CQI reporting.

- To be noted, D2D SINR distribution curve is established at a D2D user based on the history of D2D SINR/CQI measurements. To ensure the valid historical information, a sliding-window in a time domain can be applied for processing the measurement results.



FIGURE 16 Derivation of a relative threshold for CQI reporting

- D2D user would assess the measured value against the determined SINR threshold value each time. Only if the measured value is higher than the threshold, the D2D user would send CQI report. It also means that D2D users would only report the top 30\% CQIs of the history for scheduling.

- As long as eNB received the D2D CQI report, eNB would prioritize scheduling of the D2D users for data transmission, which actually implies 30\% scheduling activity for D2D users as planned. 


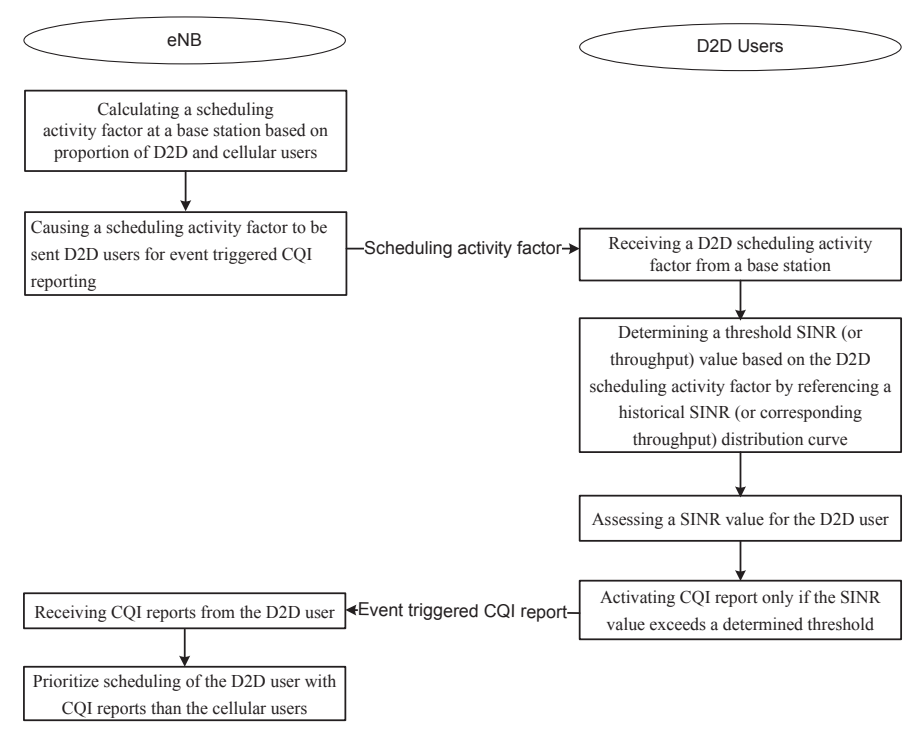

FIGURE 17 Flow chart for enhanced scheduler with optimized D2D CQI reporting

It should be noted that the threshold value of SINR derived from the scheduling factor for triggering CQI report can be changed frequently due to the updating of SINR CDF with the addition of the latest measurements. However, the relative threshold determined by the scheduling factor is not changed unless a new scheduling factor is signaled to D2D user.

In summary, there are several advantages for the proposed scheduling algorithm:

- D2D users just need to send a CQI report with the relative best channel condition in its history, which saves a lot of $\mathrm{PUCCH} / \mathrm{PUSCH}$ reporting resources and reduces UE power consumption.

- The scheduling fairness between D2D users and cellular users are secured and overscheduling of D2D users caused by the conventional PF scheduling can be avoided.

- The D2D performance is also guaranteed since eNB scheduling follows the D2D CQI reports representing the relative best channel conditions in the history.

\subsection{Semi-autonomous D2D operation}

As briefly discussed in Section 3.3.3, there are several ways to perform resource allocation for D2D operation. In article PVIII, semi-autonomous D2D operation is proposed to avoid $\mathrm{c} 2 \mathrm{~d}, \mathrm{~d} 2 \mathrm{~d}$ and $\mathrm{d} 2 \mathrm{c}$ interference more efficiently while maximizing the spectrum efficiency.

The key components of the procedure are listed in TABLE 4. 
TABLE $4 \quad$ Key components of Semi-autonomous D2D procedure

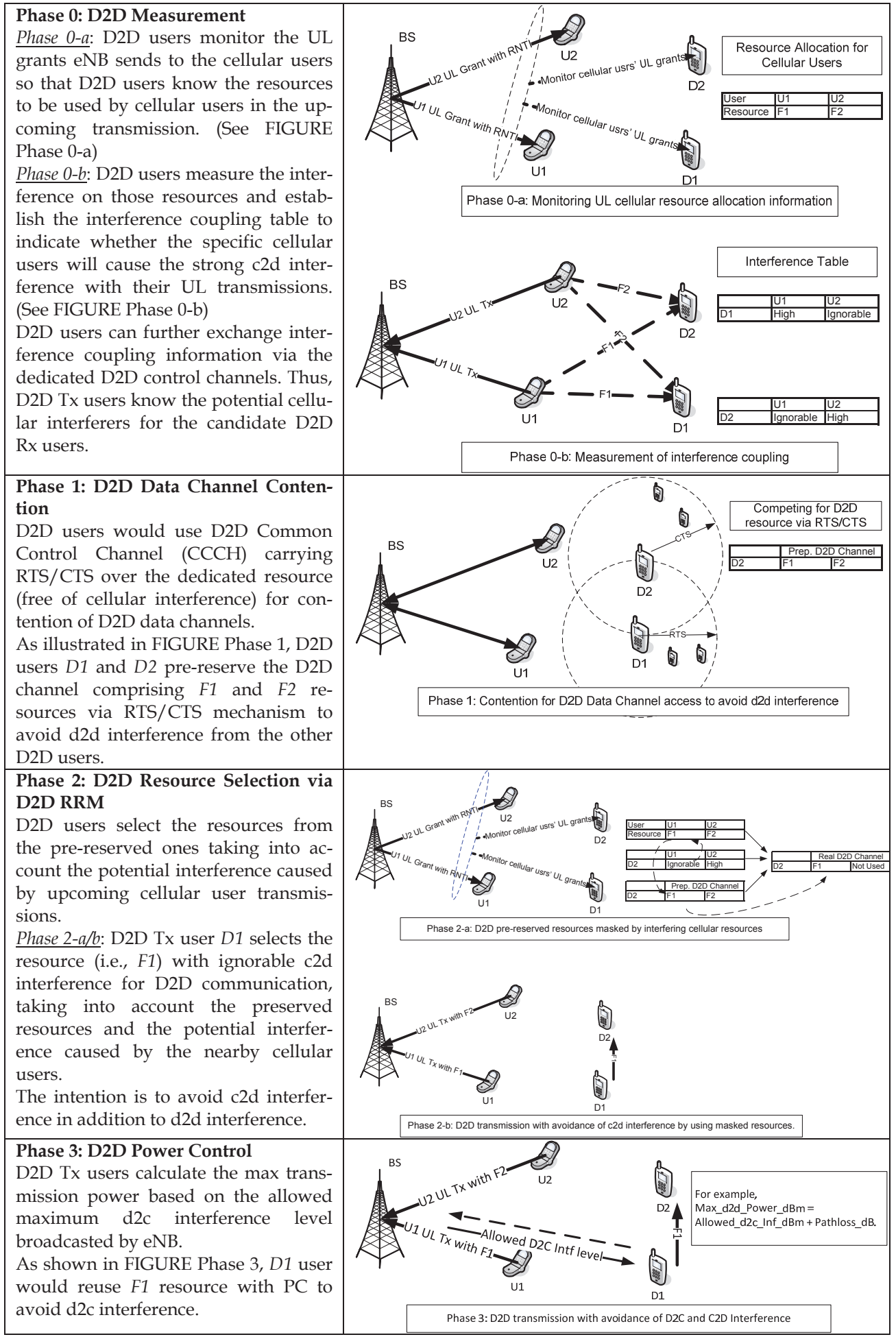


In addition, some new channels have to be introduced as below to support semi-autonomous D2D operation:

- Common Control Channel (CCCH):

- $\mathrm{CCCH}$ is used by the D2D pair to pre-reserve the resources for D2D data transmission by using RTS/CTS based mechanism for contention of D2D resources among D2D users.

- The dedicated resources are preferred for avoidance of cellular interference.

- $\mathrm{CCCH}$ channels can be operated in FDM/TDM/CDM mode.

- Data Control Channel (DCCH):

- DCCH is used to carry the data control information such as ACK and MCS information.

- DCCH can be allocated with the dedicated resources to ensure no collision and interference with other D2D and cellular communication.

- Data Traffic channels (DTCH):

- DTCH is used to carry the data traffic.

- Reusing the UL cellular data resources to improve the spectrum efficiency and share the same Tx chain as cellular transmission.

As an example, the resource allocations for these D2D channels within the cellular frequency band can be performed as FIGURE 18

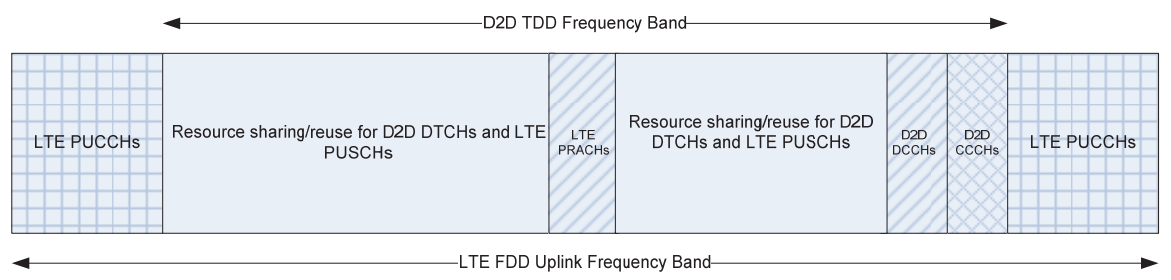

FIGURE 18 Illustration of resource partition for D2D TDD channels within cellular FDD UL spectrum

\subsection{Asymmetric D2D operation}

In the most prior arts, D2D communication requires that UEs in the D2D communication should be D2D capable with additional D2D transmitter or receiver equipped, i.e., only D2D UEs themselves can communicate with each other. It may worsen the end user experience since they always have to find another D2D capable UE for experiencing this new feature, which would restrict the D2D application especially during the ramping up phase. Furthermore, the less usage of D2D application due to such restrictions may also slow down D2D application deployment. 
Different with the symmetrical communication mentioned above, the asymmetrical D2D communication mode is proposed in [Chen13], for which the legacy UE with software updates can also communicate with a D2D capable UEs. It also paves a way for the evolution of D2D communication with the backwards compatibility and compelling performance. The proposed solution is aimed at D2D communications that is compatible with existing devices and fully utilizes them to ramp up D2D business, by giving easy experimentation for the end user. Experimentation relates to the extent to which the product or service can be experimented with a low threshold. Easy experimentation possibilities of the end-users would enhance the D2D technology diffusion.

For the asymmetric D2D communication, it assumes the cellular network operating in FDD duplex mode in which D2D connections utilize the TDD duplex mode with the cellular network UL resources controlled by the base station(s). In this case, the LTE D2D user can easily find the neighbor D2D users based on discovery via self-broadcasting or eNB assistance. Further, the LTE D2D user can decide the MCS by themselves even without eNB involvement.

Even though the legacy UEs without a UL receiver cannot receive the message from D2D UEs, they can transmit the data by reusing existing UL transmitter to D2D UEs. As illustrated in FIGURE 19, the legacy UEs without extra UL receiver can transmit the data to D2D devices with a UL transmitter and receive the D2D data via a conventional cellular link by BS forwarding. For example, the cellular device would transmit the data to a D2D device and receive the feedback or data from a D2D device via BS forwarding. In this case, HARQ feedback delay would be different than the symmetric D2D communication, i.e., the UE would take additional 4ms TTI to receive HARQ delay than symmetric communication.

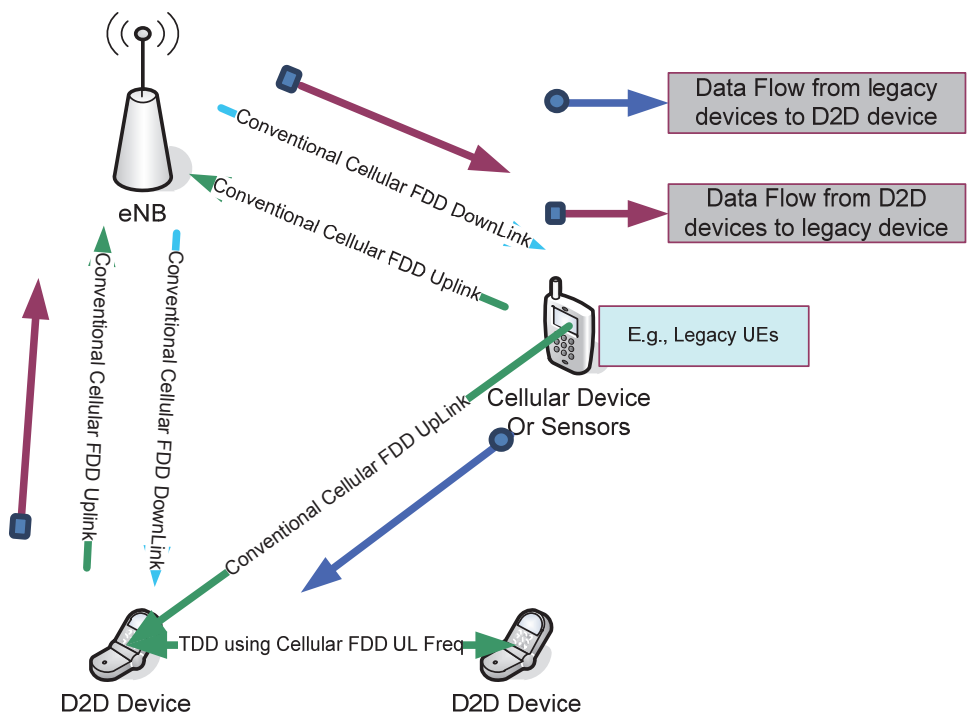

FIGURE 19 Asymmetric D2D communication between a D2D device and a legacy device 
So the legacy UE may operate in the cellular mode or the asymmetric D2D mode based on explicit signaling or implicit signaling for the parameter reconfiguration. In principle, the mode operation can be transparent to the legacy UEs. Two types of implementations could be considered:

1) Explicit D2D signaling: (new signaling would be introduced which would require software updates for the legacy UEs)

Legacy UE would receive the mode operation command (a new signaling) from eNB to indicate whether it is an asymmetric communication to the D2D device. Upon receiving the command, legacy devices may automatically reconfigure the parameters (e.g., HARQ buffer due to different ACK/NACK delay performance for cellular mode and asymmetric D2D mode) according to the pre-defined parameter settings associated with the operation mode.

2) Implicit D2D signaling: (backwards compatible by reusing existing signaling without any need for software updates)

For the mode switching of the legacy UEs between the asymmetric communication and cellular communication, it would receive the conventional reconfiguration signaling from eNB with a set of updated parameters adapting to the new operation mode.

For D2D UE, a new set of parameter settings adapting to the requirements of the asymmetric D2D communication can be indicated via explicit D2D control signaling. Upon receiving the explicit D2D signaling from eNB, D2D UE would reconfigure the system according to the pre-defined settings associated with the operation mode.

From an eNB perspective, there are several new functions to be introduced:

1) To support an asymmetric D2D operation mode in addition to symmetric D2D mode, eNB should be able to know the communicating UEs' capabilities and make the decision on the operation mode based on the following criteria:

a. If both devices are cellular devices, the cellular communication is selected.

b. If both devices are D2D devices,

i) If both devices can fulfill the D2D mode channel condition criteria, the symmetric D2D communication is preferred.

ii) If only one device can fulfill the D2D mode channel condition criteria, the asymmetric D2D communication is preferred.

c. If one device is the cellular device and the other is the D2D device, asymmetric D2D communication is preferred once the link quality criterion is reached.

2) eNB may schedule the cellular device to send the probing signal by reusing UL sounding signal while asking the D2D device to monitor the transmission, which is used to check whether the quality of the link from the legacy device to the D2D device. Based on the measurement report from the D2D device and an eNB measurement on the UL 
sounding signal, the eNB can confirm the feasibility of a connection for asymmetric communication.

3) eNB would inform the D2D device and the cellular device via explicit or implicit mode operation signaling. Meanwhile, eNB would configure itself for forwarding the messages from the D2D device to the cellular device.

The procedure is further explained in FIGURE 20, which consists of phase 1 and phase 2, as described below:



FIGURE 20 Example of asymmetric D2D communication procedure with explicit mode selection signaling for legacy UE

Phase 1: asymmetric D2D communication setup

Step 0: Once (legacy or D2D) devices has registered in the network, they would be in an IDLE state and eNB can establish a registration table with UE capability and identity information to assist D2D communication setup later. 
Step 1: When a legacy device is calling a D2D device in the same cell, eNB would check the table and find if it is possible to have an asymmetric D2D communication. Then eNB would trigger the initial access procedure between two devices to check the connectivity.

Step 2: eNB can configure UL sounding transmission for the legacy device. Accordingly, eNB would ask D2D device for the measurement according to measurement control message.

Step 3: When the cellular UE is sending a UL sounding signal as in the cellular mode, the D2D device would measure it and send the measurement report to eNB.

Step 4: if the asymmetric communication criteria are fulfilled according to the measurement report from D2D device and eNB measurement, eNB can confirm the connectivity is OK.

Step 5: eNB would send D2D asymmetric mode command to the legacy device and the D2D device separately to imply that the legacy UE is communicating to the D2D device in an asymmetric D2D communication mode. Accordingly, the devices would reconfigure their protocol entities with the pre-defined parameter settings.

Step 6: eNB would help to complete the D2D communication setup, e.g., allocating the common RNTI for a pair of devices or informing the devices the peer's RNTI.

Phase 2: asymmetric D2D communication

Step 1: During the transmission, eNB would firstly grant UL resources to the legacy UE using the pair RNTI. Meanwhile, the D2D device can also decode it and re-interpret it as the information about how to receive the packets from the legacy UE.

Step 2: Depending on the packet reception, the D2D device may send ACK/NACK feedback message to the legacy UE via eNB forwarding. Then the retransmission or new transmission can be performed accordingly.

Note: In order to maintain the synchronous HARQ for the legacy UE, BS forwarding should not lead to some big variation in the delay, which would impact the HARQ buffer configuration for the legacy UE.

During the asymmetric communication, the transmission power of legacy UEs can be adapted to the D2D link by eNB PC commands in order to save power and reduce interference. There are several advantages for the proposed scheme:

- The legacy UEs can be fully reused to support the D2D feature with little or no modification. It can enrich D2D use cases and applications quite much.

- It is not mandatory for the end user with the D2D device to find a peer device with the D2D feature for the D2D application. Any D2D device can have good user experience regardless of the peer device. 
- Even though both devices are D2D, it is still possible to apply the asymmetric D2D communication to adapt to the channel conditions of each device.

- It also provides the possibility for the legacy UE to broadcast the information to a group of D2D UEs. Furthermore, if no feedback is required for this kind of broadcasting service, the implementation could be further simplified for the legacy UEs.

- The legacy UE can be taken as a downlink relay to forward the eNB messages to D2D UEs. (e.g., the legacy UE receives the messages from eNB in DL band and transmits to D2D UEs in UL band.)

- The asymmetric D2D communication complements the symmetric D2D communication as a more integrated D2D concept, which can be beneficial for both D2D devices and legacy devices.

- Easy experimentation possibilities of the end-users by fully considering the backwards compatibility of the devices can enhance the D2D technology diffusion.

\subsection{Feedback assisted D2D multicast operation}

The increasing interest of offering a multicast service over the cellular system has been driving the development of the efficient and cost-effective solutions in the industry standards. Multimedia Broadcast Multicast Services (MBMS) is one of the specifications in existing 3GPP cellular networks to support point-tomultipoint communication, which is designed to provide efficient delivery of broadcast and multicast services, both within a cell as well as within the core network. Target applications include mobile TV and radio broadcasting, as well as file delivery and emergency alerts.

With the introduction of D2D operation, D2D cluster based multicast operation is emerging, which enables user-centric based low-cost multicast services rather than the conventional infrastructure network based services. It also provides the possibility of group communication even without network coverage. With multicast directly within D2D cluster, one hop transmission without involvement of eNB can significantly save both time and network resources. Moreover, the multicast need not be limited to the D2D mode. With modifications to the base station, multicast can be used in the cellular mode also, providing service continuity to enhance the data sharing in cellular networks.

Due to the nature of the wireless radio channel, link quality can be dramatically different for each receiver, even if they were located geographically close to each other. In unicast the problem would be mitigated by using a different transfer rate for each, depending on the channel quality. In multicast, transmitting to each receiver at different rates would add to the system's complexity and in many cases be impractical. For example, were a streaming video sent to one receiver at a very high transfer rate, and at a lower rate to another, 
the first receiver would become subject to unacceptably long delays waiting for the second one to catch up. Thus it will become a challenging task to choose an optimal transfer rate for the multicast transmission.

The typical way of solving the problem is to use the worst link's transfer rate for every receiver. This way performance suffers for users with good link quality, but it is ensured that every user receives the data correctly. Due to this, it is important that the mode selection between D2D and cellular is done properly. Thus, in reliable multicast one of the key design challenges is the feedback. It should be noted that Feedback implosion is a known problem (e.g., in [Amm97]).

In this section, we will focus on the feedback assisted D2D multicast operation, which was investigated in article PIX where some simulation results are provided for verification of the D2D clustering concept.

\subsubsection{HARQ Feedback on common resources}

Located on the medium access control (MAC) layer, HARQ is the lowest level of error correction in LTE. In cluster multicast, HARQ level feedback is handled by joining every receiver's feedback messages into a common feedback region. The selected scheme is a NACK based feedback scheme where only an incorrectly received transmission is indicated back to the transmitter. If a receiver receives the packet correctly it will not send any feedback. The multicast HARQ scheme is depicted in FIGURE 21, in which feedback for each HARQ process is mapped to the different time instants whereas the same frequency resources are used for the feedbacks from multiple users corresponding to the same HARQ process.

In this case, the NACK feedback can be softly combined to improve the detection performance while saving the resources. On the other hand, from the transmitter perspective it cannot identify the specific failure at the receiver(s) due to the common feedback so that link adaptation and power adjustment cannot be effectively exploited for improving the (re-)transmission performance for a specific failed receiver.

It should be noted that cooperative retransmissions can be performed in this case in which the successful receivers in the previous (re-)transmissions can forward the received packets to the nearby failed receivers. However, it is likely a kind of multipoint-to-multipoint retransmission, which is similar to Single Frequency Network (SFN) based multicast operation in the cellular network. In other words, it can be considered as a kind of blind cooperative retransmission.

Another constraint for this scheme is that there is no explicit ACK feedback. In some cases, the successful transmission cannot be separated from the failed transmission caused by the coverage limitation. Thus, such resource mapping schemes may require the full connections between the cluster members, which, however, can be a fair assumption considering the short distance between D2D users within the cluster. 


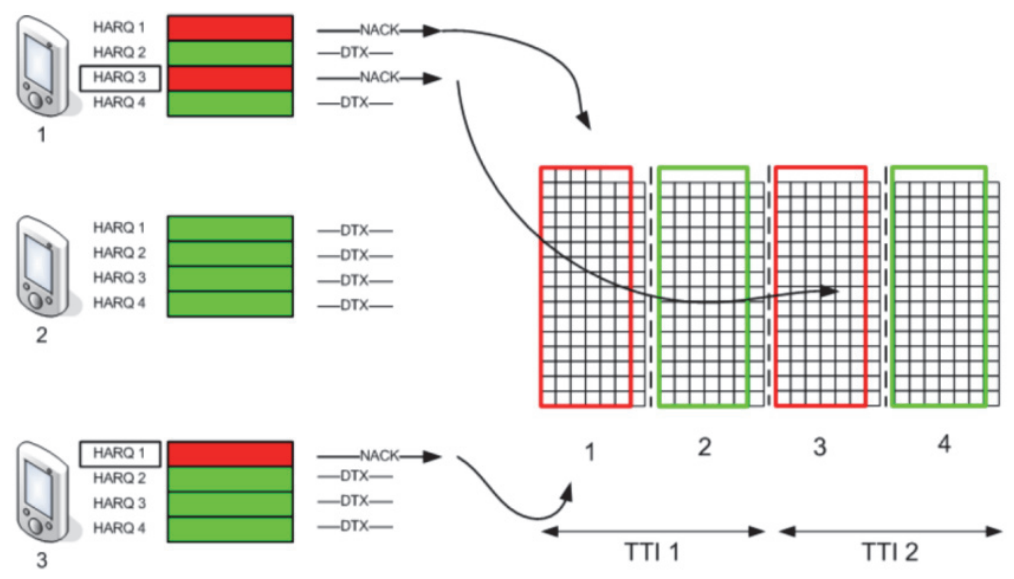

FIGURE 21 HARQ feedback mapped to a common resource

\subsubsection{HARQ feedback on user specific resources}

The other option is to individually decode the NACKs with user specific sequence like cyclic shift CAZAC sequence (see [4G211]) and Walsh-Hadamard codes (see [3G211]). Also, both techniques can be used in tandem. For example, the NACKs for the first $N$ transmissions would be softly combined since a lot of users may fail during the period. Then NACKs for the last $M$ transmissions could use the user specific sequence to inform the transmitting device which user still failed.

With the help of the individually coded NACKs, the transmitter will know exactly which receivers perform worst in the long term. This information could also be used for intelligent cluster group management. For example, the receiver which cannot provide any ACK feedback for a certain number of transmissions, it would be deemed as out-of-coverage and then dropped from the D2D cluster.

Similarly, cooperative retransmission can be applied more effectively based on the user specific feedback. Each user can measure the signal strength of the user specific NACK message to derive the pathloss in between. This information can be reported to the eNB so that eNB can assign the specific user (e.g., the nearby user) to retransmit the successful received data for the failed ones. This user specific cooperative retransmission is more efficient than the blind cooperative retransmission.

\subsection{Performance evaluation}

\subsubsection{Simulation setup and performance analysis for RRM functions}

The main simulation and LTE system parameters, which are either alternative or differ from those defined in LTE specifications, are given in TABLE 5. If the 
location of a D2D Rx user dropped into the network layout did not lead up to the D2D operation mode, the user was relocated until the D2D mode was chosen. This procedure is similar to the widely used load balancing approach that was also used here for the cellular and D2D Rx users. These procedures enable the study of the effects of D2D communication with a fixed number and proportion of cellular and D2D users per cell. Without loss of generality, we assume the traffic in D2D links to be unidirectional within a simulation drop.

The cellular layout is a wrapped-around hexagonal grid with 19 base station sites and 3 sectors per site. Both the interference limited scenario (3GPP Case 1) and the cover limited scenario (3GPP Case 3) as defined in [4G814] are adopted for an extensive performance evaluation, in which the inter-site distance (ISD) between eNBs is $500 \mathrm{~m}$ corresponding to an interference limited macro-cell scenario and $1700 \mathrm{~m}$ for a coverage limited macro-cell scenario. There is a balanced load of $N_{c}$ cellular users and $N_{t x}$ D2D Tx users per sector, that is, altogether $N_{c}+N_{t x}$ Tx users per sector. The total number of Tx users is fixed with 8 users in the study with a varying number of $N_{t x}$. The cellular users and D2D Rx users have random locations uniformly distributed over the whole network under the load balancing condition. Each of the D2D Tx users is given a random uniformly distributed location over a disc with a given maximum radius $r_{\max }$ around its pairing D2D Rx user. The value of $r_{\max }$ was set to be 400 $\mathrm{m}$, which is well above the maximum MS-BS distance of $500 / \sqrt{3} \approx 289 \mathrm{~m}$ in Case 1 scenario, in order not to artificially limit the D2D link distances. There is no lower limit for the distance between mobiles, whereas for the MS-BS distance the limit is $35 \mathrm{~m}$.

TABLE 5 Simulation parameter settings

\begin{tabular}{ll}
\hline \hline Parameter & VALUE \\
\hline User deployment & Total 8 D2D/cellular Tx users per cell \\
$N_{t x}$ & $0,2,4,6,8$ D2D Tx users \\
Macro cell deployment & 3GPP Case 1 with 500m ISD; \\
& 3GPP Case 3 with 1732m ISD \\
Minimum MS-BS distance & $35 \mathrm{~m}$ \\
Carrier frequency & $2 \mathrm{GHz}$ \\
Bandwidth & $10 \mathrm{MHz}$ \\
Power control & $P_{0}=-56 \mathrm{dBm}, \alpha=0.6$ \\
Modulation scheme & BPSK, QPSK, 16-QAM, 64-QAM \\
Coding rate & $1 / 3,1 / 2,2 / 3,3 / 4$ \\
BLER target & $20 \%$ after 1 st transmission \\
Max. retransmissions & 3 \\
Channel estimation & Non-ideal \\
Traffic model & Full buffer \\
Scheduler & Proportional fair \\
Resource allocation & 6 PRBs per Tx user \\
UE transmit power & 24 dBm \\
\hline \hline
\end{tabular}




\subsubsection{Performance for the interference limited scenario}

As investigated in article PVI, for the interference limited scenario with D2D link based D2D PC, when the system operation shifts from cellular to D2D connections, the mean throughput of all users gradually increases with D2D share of the total system load as the inter-cell interference level decreases due to D2D lower transmission powers. For the same reason, the cell edge performance at $5 \%$ -tile also benefits as the load shifts from cellular to D2D links with less inter-cell interference. For the cases using the cellular link based D2D PC, both the mean throughput and cell edge throughput over all users gradually increases with D2D share of the total system load. However, the cell edge performance is significantly improved for the case with D2D link based PC than cellular link based PC thanks to the low transmit power. On the other hand, the relatively low transmission power with D2D link based PC may result in the slightly lower mean throughput as the power setting based on the D2D pathloss does not have any margin to overcome any extra inter-cell co-channel interference. In this interference limited scenario, performance is more sensitive to the setting of the transmission power which may increase the interference while enhancing the signal strength. Essentially, the gain mechanism is quite different for D2D link and cellular link based PCs although both of them can improve the systems overall performance. In practical operation, the selection of power control scheme may also depend on the goal of the network optimization.

More results and analysis can be found in article PVI.

\subsubsection{Performance for the coverage limited scenario}

Similarly to the performance in the interference limited scenario, both the mean throughput and the cell edge throughput over all UEs are significantly improved compared to the baseline without any D2D operation thanks to the contribution of D2D operation. In addition, it seems no clear performance difference for the cases with the maximum D2D distance of $50 \mathrm{~m}$. Also for the case with a maximum $400 \mathrm{~m}$ D2D distance, the overall performance is much lower than the case of D2D operation with a maximum 50m distance. The larger allowed D2D operation range would require a much higher transmission power as proved. In this coverage a limited scenario with the larger D2D operation range, the overall performance is more limited by the affordable transmission power to improve the signal strength rather than the co-channel interference.

With only D2D links, there is no notable difference between the two PC schemes. This is because the inter-cell interference is more contributed by D2D transmission rather than the cellular transmission. Then the high transmission power with cellular link based PC would likely lead to more interference, thus slightly or even no improvement in performance compared to D2D link based PC. For the throughput of cellular links, it should be kept the same thanks to the same interference level caused by the unchanged transmission power for the D2D mode and cellular mode with cellular link based PC. Eventually, the overall performance is improved significantly.

More results and analysis can be found in article PVI. 


\subsubsection{Performance for D2D mode selection}

Due to the significance of mode selection for proper D2D operation, more studies have been done in [Hak10] to investigate the performance of several mode selection algorithms against the derived upper bounds, assuming code multiplexing access for D2D pairs, similarly to WCDMA system.

The key parameters for simulations have been captured in TABLE 6, in which the probability of occurrence for D2D service and the maximum distance for D2D communication have been taken into account.

TABLE 6

Simulation parameter settings

\begin{tabular}{|l|l|}
\hline \multicolumn{1}{|c|}{ Parameter } & \multicolumn{1}{c|}{ Value } \\
\hline Cell configuration & Single cell \\
\hline Cellular link pathloss model & PL=128.1+37.6*log10(R_km) [G3003] \\
\hline D2D link pathloss model & PL=148+40*log10(R_km) [G3003] \\
\hline Shadow fading standard deviation & $\begin{array}{l}10 \mathrm{~dB} \text { for cellular mode links; } \\
12 \mathrm{~dB} \text { for D2D mode links. }\end{array}$ \\
\hline Minimum coupling loss & $\begin{array}{l}70 \mathrm{~dB} \text { for BS-Device; } \\
40 \mathrm{~dB} \text { for Device-Device. }\end{array}$ \\
\hline Processing gain & $\begin{array}{l}30 \text { for cellular mode; } \\
15 \text { for D2D mode. }\end{array}$ \\
\hline $\begin{array}{l}\text { Probability of local communication } \\
\text { per device }\end{array}$ & $20 \%, 40 \%$ \\
\hline $\begin{array}{l}\text { Maximum distance between locally } \\
\text { communicating devices }\end{array}$ & $5 \mathrm{~m}, 50 \mathrm{~m}$ \\
\hline
\end{tabular}

As shown in TABLE 7 summarized from the simulation results in [Hak10], it can be seen that when the maximum D2D distance is quite large $(50 \mathrm{~m})$ there is not much potential improvement available from D2D capability in the system. The available performance improvement increases as a function of increasing local communication probability. On the other hand, we can see how dangerous it would be to use path loss based or forcing all locally communicating devices into D2D communication mode in this case. These methods really deteriorate the systems performance and actually as local communication probability increases the situation becomes even worse. This is due to the fact that these methods do not consider the interference situation at all.

TABLE 7

System capacity - Number of active links per cell

\begin{tabular}{|l|l|l|l|l|}
\hline Max D2D distance & \multicolumn{2}{|c|}{ 50m } & \multicolumn{2}{c|}{ 5m } \\
\hline D2D communication probability & $\mathbf{2 0} \%$ & $\mathbf{4 0} \%$ & $\mathbf{2 0} \%$ & $\mathbf{4 0 \%}$ \\
\hline Cellular Mode (UEs/Cell) & 40 & 40 & 40 & 40 \\
\hline Opt D2D (UEs/Cell) & 43 & 50 & 50 & 63 \\
\hline Pathloss-based D2D (UEs/Cell) & 28 & 25 & 50 & 61 \\
\hline Forced D2D (UEs/Cell) & 13 & 9 & 50 & 62 \\
\hline
\end{tabular}

However, when the maximum distance is 5 meters, even path loss based and forced D2D methods behave quite well, almost reaching the achievable upper bound. In addition to gains with optimal mode selection method, gains 
with two other mode selection methods increase as the local communication probability increases.

In some sense, these observations are also aligned with some findings in Section 3.7.1.1 and 3.7.1.2 for scenarios with co-channel interference limited or not. From the above observations one could think that a practical mode selection algorithm could be based on interference- awareness in which interference measurements from devices could be used for mode selection especially in the scenarios with significant co-channel cross-link interference among devices.

\subsubsection{Simulation setup and results analysis for time hopping scheme}

To simplify the simulation without loss of generality, the performance of the random TH without ICI is investigated. A quasi-static simulator is established for the evaluation of the performance with multiple drops. The cell layout is shown in FIGURE 22. D2D Tx UEs are evenly distributed in each cell, and Rx UEs are located around the Tx UEs with a pre-defined maximum distance (i.e., D2D circle radius). LTE FPC is used as UL power control scheme for D2D users, which can prevent the eNB receivers from the excessive D2D interference. Note that only one frequency resource is modeled.



FIGURE 22 Cell layout with several D2D circles per cell, each D2D circle (dot line circle) including one Tx UE (solid dot) and one Rx UE (gray circle)

Main parameters used in the system simulation are summarized in TABLE 8. 
TABLE 8 Simulation parameter settings

\begin{tabular}{|l|l|}
\hline \multicolumn{1}{|c|}{ Parameter } & \multicolumn{1}{c|}{ Value } \\
\hline Inter site distance & $0.5 \mathrm{~km}$ \\
\hline Cell configuration & 19 cells \\
\hline Cellular link pathloss model & PL=128.1+37.6*log10(R_km) \\
\hline D2D link pathloss model & PL $=127+30^{*} \log 10\left(\mathrm{R} \_\mathrm{km}\right)$ \\
\hline Cellular UEs per cell & $1 \mathrm{UE}$ for case A; 0 UE for case B and C. \\
\hline D2D pairs per Cell & $3,6, \ldots, 18$ pairs \\
\hline Max D2D circle radius & $20,40, \ldots, 200 \mathrm{~m}$ \\
\hline TH groups (NG) & $3,6, \ldots, 18$ \\
\hline TH period (NP) & $1,2,3,4,8,16,32$ \\
\hline Time length for non-TH & 32 \\
\hline Cellular link PC setting & FPC $\left(\alpha=0.6, P_{0}=-56 \mathrm{dBm} / \mathrm{RB}\right)$ \\
\hline D2D link PC setting & FPC $\left(\alpha=0.6, P_{0}=-56 \mathrm{dBm} / \mathrm{RB}\right) ;$ \\
\hline
\end{tabular}

\subsubsection{Effect of near-far interferers}

Firstly, it is worth checking the impact of near-far interference on the performance. With increasing max D2D radius, the weakness on the wanted signal due to the increased pathloss and relatively increased near-far interferers would make the performance worse. When the number of D2D pairs is quite small on the allocated frequency resource, the performance difference between reusing and not reusing the same frequency resource with cellular users are quite considerable. In this case, the interference from intra/inter cell cellular users dominate. On the other hand, increasing the reusing D2D pairs, the interference from D2D pairs is prevalent, thus obscuring the performance difference on the cases $\mathrm{w} / \mathrm{o}$ reusing with cellular users.

More results and analysis can be found in article PVII.

\subsubsection{Effect of TH on the performance}

The performance regarding to the mean SINR received by D2D UEs with TH is quite similar to that without TH. However, the Std SINR values with TH are far below the reference cases without TH. Increasing the resource group number, the difference on the reduction of Std SINR is not significant any more.

Furthermore, the SINR distribution can exemplify the improvement on the performance by TH for the worst D2D UEs that are heavily interfered by nearby D2D pairs. The reason for the gain from $\mathrm{TH}$ is such that, it can reduce the D2D interference efficiently by exploiting the characteristics of ideal orthogonality in the time domain to randomize the interference from neighbor transmitting UEs. Besides, it is expected to obtain more gain via fully orthogonal TH sequences allocated to mutual interfering D2D pairs.

In short, the gain from $\mathrm{TH}$ is quite general and holds for most scenarios regardless of the D2D distance and traffic load. However, the specific gain may depend on the parameters settings.

More results and analysis can be found in article PVII. 


\subsubsection{Effect of TH parameters on the performance}

In this section, two key parameters of $\mathrm{TH}$, the time resource group number and $\mathrm{TH}$ period, are investigated to provide a guideline for the operation of $\mathrm{TH}$.

More resource groups in the time domain can reduce the co-channel interference significantly, thus achieving the higher SINR via the ideal orthogonality in the time domain. On the other hand, it makes performance more sensitive to the co-channel interference in the high SINR region where any access collision would deviate SINR far away from the mean value. In addition, the simulation results indicate that the large grouping number can improve the mean SINR but deviate SINRs heavily, especially in the case that there are only a few D2D pairs.

However, increasing the number of resource groups in the time domain, $\mathrm{TH}$ can enlarge the gain in terms of the reduction on Std SINR. It is because TH can efficiently randomize some occasionally happened collisions compared to the reference cases. Besides, it can be noted that more groups may result in a larger transmission delay since the D2D user has to wait longer for the next transmission.

In general, the results provide some hints for the optimal configuration, i.e., increasing the grouping number can improve mean SINRs, whereas reducing TH period can improve Std SINRs. Thus, the adjustment of the parameters depending on QoS, delay requirements and D2D load may be an efficient way to optimize the performance.

More results and analysis can be found in article PVII.

\subsubsection{Simulation setup and results analysis for semi-autonomous D2D}

In this section, some simulation results are provided for evaluation of semiautonomous D2D described in Section 3.4.

The topology of the hybrid system will be modeled in a fashionable way where D2D users are distributed in a randomly placed cluster with a radius of $10 \mathrm{~m}$ and cellular users are also dropped uniformly through the cell with a radius of $300 \mathrm{~m}$. Such a topology is more realistic in modeling urban environments [Kau08]. There are altogether $50 \mathrm{D} 2 \mathrm{D}$ users placed in the system with regards to the number of cellular UEs changing from 50, 100, 200 to 2000. Considering the short distance between a D2D pair and the large radius of the cellular system, a simple power control scheme is utilized by letting the transmission power of TxD_UE 15dB lower than that of cellular users. The used system bandwidth is $1.4 \mathrm{MHz}$, i.e., $6 \mathrm{RBs}$ altogether. By reserving $2 \mathrm{RBs}$ for the $\mathrm{CCCH}$ and $\mathrm{DCCH}$ of a D2D subsystem, the left 4 RBs are available to transmit data for both networks. The adopted threshold by cellular UEs to determine if D2D users are adjacent to them is SINR which is received from $\mathrm{CCCH}$ and DCCH.

The other parameters are set up according to [3G814] and are presented in TABLE 9. 
TABLE 9 Simulation parameter settings

\begin{tabular}{|l|l|}
\hline \multicolumn{1}{|c|}{ Parameter } & \multicolumn{1}{c|}{ Value } \\
\hline Cell Radius & $300 \mathrm{~m}$ \\
\hline Cellular link pathloss model & $\mathrm{PL}=128.1+37.6^{*} \log 10\left(\mathrm{R} \_\mathrm{km}\right)$ \\
\hline D2D link pathloss model & $\mathrm{PL}=127+30^{*} \log 10\left(\mathrm{R} \_\mathrm{km}\right)$ \\
\hline Max D2D circle radius & $10 \mathrm{~m}$ \\
\hline Max Tx Power of eNB & $46 \mathrm{dBm}$ \\
\hline Max Tx Power of Cellular UE & $24 \mathrm{dBm}$ \\
\hline Max Tx Power of D2D UE & $9 \mathrm{dBm}$ \\
\hline
\end{tabular}

To avoid interference between D2D and cellular UEs when sharing the same resources, a threshold for the interference level or SINR measured by the victim UE is set to check whether the Tx UE(s) causes significant interference for the establishment of the interference coupling matrix. As indicated in the results from article PVIII, this threshold plays a critical role for the semiautonomous D2D performance. If the threshold is set higher, e.g., $6 \mathrm{~dB}$ (i.e., at least $6 \mathrm{~dB}$ higher interference received by the victim $\mathrm{UE}$ ), then the gain from interference avoidance mechanism will be vanished. As expected, the highest gain is observed with the threshold setting at $-3 \mathrm{~dB}$. However, further reducing the threshold setting may limit the opportunities for sharing the resources unnecessarily, which may result in shrinkage of the system performance gain. Additionally, the smaller threshold setting may trigger more interference reports due to sensitivities to the interferences from near-by UEs, which may cause the overhead of signaling. Thus, the threshold for interference avoidance should be set carefully by taking into account the tradeoff between the performance and the signaling overhead.

More results have been presented in article PVIII to investigate the impact of the threshold setting with varying maximum D2D distance, cellular UE density and partition of time resources between D2D and cellular UEs.

In general, it can be noticed that the interference avoidance mechanism, reusing the same resources according to the interference coupling matrix established from interference measurements, can effectively improve the resource efficiency, accordingly enhancing the performance of the whole system.

\subsection{Conclusion}

In this chapter, we presented a system concept of D2D communication for the long term evolution of 3GPP LTE-Advanced network system to address the fast increasing local traffic. The performance of direct D2D communication in an LTE system over an interference limited micro-cellular environment and a coverage limited macro-cellular environment was evaluated. The D2D operation deployed the uplink band of the LTE FDD mode and took place between two same-cell users that had higher received power in their mutual link than in those towards the base station. The simulation results showed that besides re- 
moving the duplicated traffic from the downlink with additional downlink resource saving, D2D transmission can improve the overall system throughput also in the uplink. Since receiving D2D users are susceptible to interference from nearby high-power users that are scheduled on the same resources, their throughput can be greatly improved by allowing them to use higher cellular link based transmission power instead of D2D link based power when the majority of other users in the network are conventional cellular users. The lower D2D transmission powers, on the other hand, reduce the interference level and, therefore, improved the throughput of cellular users as the system load shifts from cellular to D2D links. Finally, the reuse of cellular resources for D2D communication, even when done so blindly, was demonstrated to provide further improvement in the system throughput.

Moreover, the semi-autonomous D2D scheme is also presented with the interference avoidance mechanism to mitigate the interference among D2D and cellular UEs. In practical operation, the threshold setting should be performed carefully considering the tradeoff between system performance and signaling overhead.

In addition, time hopping of D2D resources within cell and across cells is carried out to randomize near-far interference from nearby transmitting D2D UEs and also nearby transmitting cellular UEs, which can reduce problematic near-far interference in D2D communications efficiently. The gain from $\mathrm{TH}$ hopping is not limited to the specific cases. The optimization of the TH parameter settings can further improve the overall performance depending on the service requirements. Moreover, it is easy to operate TH because of the low signaling overhead and a set of limited choices for the parameters optimization. 


\section{SUMMARY}

With fast growing traffic and rich applications, one of the targets for network evolution is to ensure that the growing infrastructure investment to fulfill the demands of the exponential growth of mobile traffic can turn to the linear increase of the revenue. The low cost operation with effective solutions to support diverse application is critical to bust the myth of the scissor effect, i.e., the revenue falls apart from the increase in traffic. Two types of typical services are considered for optimization in the network evolution: voice based RT services and data based NRT services.

For the voice service, it is characterized by the high demand on the packet delay and the small size of the payload, which has been typically carried by the dedicated radio resources and transported through the circuit switched core network to secure the quality of service. With an increasing interest to use cellular networks for RT packet switched (PS) services such as VoIP and motivation of migrating CS network towards the single PS network for operation cost saving, the solutions for offering VoIP over the cellular network has been widely studied in this thesis. VoIP over E-DCH has been proved to provide the comparable performance as the legacy DCH based voice solution. Moreover, uplink DPCCH gating for E-DCH carried VoIP service can further improve the performance significantly, especially when it operates jointly with packet bundling to achieve the higher power saving performance and improve Turbo encoder efficiency. The reduction of transmission power in the downlink due to HSSCCH-less operation can also turn to the higher data throughput in case of mixed VoIP and data traffics or enhance the VoIP capacity with more scheduled users. Meanwhile, the downlink packet scheduler enhancement considering the packet age also provides the potential for the improvement of the end-to-end user experience. On the other hand, it should be kept in mind that reduction on some of the control channels, e.g., HS-DPCCH for carrying CQI reports, may cause the performance loss in the downlink due to less reliable CQI reports for downlink link adaption.

For the data service, the high efficient cellular networks and value-added services are critical for the operators to mitigate the scissor effect. Recently, LTE system with the peak data rates of DL $100 \mathrm{Mpbs}$ and UL 50Mbps has been in 3GPP for the good opportunities to fulfill the requirements of fast increasing mobile traffic. However, considering 1000 times increase in traffic and challenges of supporting MTC devices and traffic, more advanced solutions are expected. A hybrid network integrating device-to-device (D2D) communication into LTE network can be considered as one of the options to further improve the network efficiency and enable some new value-added services. Especially, D2D communication under the cellular network can reduce transmitter power consumption both in device and base station, increase cellular network capacity and establishing more services for the users. However, there are also more challenges to be solved for achieving the goal, such as the complicated co-channel interference, the near-far problem caused by the distributed transmission, ICI 
problems due to in-band emission, and scheduling fairness with mixed D2D and cellular users. Accordingly, RRM solutions for mode selection, PC, resource allocation have to be supported for a feasible D2D operation, which are further enhanced by time hopping solutions and enhanced scheduler algorithm as presented in this thesis. In general, it seems promising for D2D operation as one path of future network evolution. On top of the basic D2D operation, there are also other variants depending on the deployment scenarios and business cases, such as asymmetric D2D operation which also gives chances for the legacy UEs to participate D2D communications. Semi-autonomous D2D also provided another way for D2D implementation leveraging the performance and signaling overhead. In addition, D2D based cluster operation offers the opportunities for supporting the multicast feature in the cluster basis. With the feedback mechanism for multicast operation, it can give the chance for the cooperative D2D operation which will bring some additional benefits to improve system performance in a cost-effective approach.

In short, the VoIP based voice solutions and D2D based data solutions are the potentials for future network evolution. Especially, considering the fast increasing devices in the future with more demanding traffic including, e.g., from the services for the sensors with a few data bits to the services for carrying realtime video traffic with huge data volume, the conventional cellular network has to be evolved to support the local traffic in addition to the wide area coverage, in which D2D based wireless mesh network with the support of IP-based realtime services can be the direction for future network evolution. 


\section{YHTEENVETO (FINNISH SUMMARY)}

Tässä työssä käsiteltiin kahta tyypillistä palvelua, puhepohjaisia reaaliaikapalveluita ja datapohjaisia ei-reaaliaikapalveluita, sekä tutkittiin ratkaisuja niiden tehokkaaseen tarjoamiseen tulevaisuuden solukkoverkkojärjestelmissä. Puhepalveluiden osalta tutkimuksen kohteena oli IP puhe (Voice over IP, VoIP) HSPA järjestelmissä. Työssä kehitettiin uusia palveluja tukevia menetelmiä sekä tutkittiin niillä saatavia ylä- ja alalinkin suorituskyvyn parannuksia. Datapalvelujen osalta tutkimuksen alla oli LTE solukkoverkko, jonka toimintaan oli integroitu päätelaitteiden välinen (device to device, D2D) kommunikointimahdollisuus. Kyseisessä skenaariossa tutkittiin järjestelmän spektritehokkuutta ottaen huomioon uusia kehitettyjä radioresurssien hallintamenetelmiä. 


\section{REFERENCES}

[3G211] 3GPP TS 25.211, "Physical channels and mapping of transport channels onto physical channels (FDD)"

[3G300] 3GPP TS 36.300, "Evolved Universal Terrestrial Radio Access (EUTRA) and Evolved Universal Terrestrial Radio Access Network (E-UTRAN); Overall Description; Stage 2 (Release 8)"

[3G308] 3GPP TS 25.308, "HSDPA; Overall description; Stage2".

[3G309] 3GPP TS 25.309, “FDD Enhanced Uplink; Overall description; Stage 2"

[3G814] 3GPP TR 25.814 V7.1.0 (2006-9), “Physical layer aspects for evolved UTRA".

[3G896] 3GPP TR 25.896, "Feasibility Study for Enhanced Uplink (FDD)”.

[3G903] 3GPP TR 25.903 V1.0.0 (2006-05), "Continuous Connectivity For Packet Data Users".

[3G903] 3GPP TR 25.903 V1.0.0 (2006-05), "Continuous Connectivity For Packet Data Users".

[4G101] 3GPP TS 36.101, v8.1.0, “User Equipment (UE) radio transmission and reception", March 2008.

[4G211] 3GPP TS 36.211, v12.3.0, “TS 36.211 Evolved Universal Terrestrial Radio Access (E-UTRA); Physical channels and modulation",

[4G213] 3GPP TS 36.213 V9.3.0, “Evolved Universal Radio Access (EUTRA); Physical layer procedures"

[4G321] 3GPP TS 36.321, v8.3.0, “EUTRAN Medium Access Control”

[4G331] 3GPP TS 36.331, v8.3.0, “EUTRAN Radio Resource Control”

[4G814] 3GPP TR 36.814 V1.5.0, "Further Advancements for E-UTRA. Physical Layer Aspects".

[Ada98] T. Adachi and M. Nakagawa, "A study on channel usage in a cellular-adhoc united communication system for operational robots," IEICE Trans. Commun., vol. E81-B, no. 7, pp. 1500-1507, Jul. 1998.

[Alam14] Alam, M., Du Yang, Rodriguez, J., Abd-alhameed, R., “Secure device-to-device communication in LTE-A", IEEE Communications Magazine, 2014

[Alc08] R1-082813, “M2M Communications", Alcatel-Lucent, 3GPP RAN1\#54, 18-22 August 2008. 
[Ale11] Alegre Perez, J.P.; Pueyo, S.C.; Lopez, B.C., “Automatic Gain Control, Techniques and Architectures for RF Receivers", Springer, 2011

[Amm97] M. Ammar and D. Towsley, "Reliable multicast," SIGCOMM97 Tutorial, Sep 1997.

[Asa14] Asadi, A, Wang, Q., Mancuso, V., “A Survey on Device-to-Device Communication in Cellular Networks", IEEE Communications Surveys \& Tutorials, 2014

[AT009] AT\&T, Orange, etc, “One Voice; Voice over IMS profile", V1.0.0, 2009

[Bae13] Sueng Jae Bae, Dong Hyun Kim, Bum-Gon Choi, Min Young Chung, "Transmission Power Control for FlashLinQ Device-toDevice Communication System", IEEE 77th Vehicular Technology Conference (VTC Spring), 2013

[Ban05] W.Bang, K. I. Pedersen, T.E. Kolding, and P. E. Mogensen (2005), "Performance of VoIP on HSDPA", IEEE Proc. Ve-hicular Technology Conference, Stockholm, June.

[Bas08] Basuki E. Priyanto, Troels B. Sørensen, Ole K. Jensen, "In-Band Interference Effects on UTRA LTE Uplink Resource Block Allocation", VTC spring 2008.

[Chen12] Xiaohang Chen, Li Chen, Mengxian Zeng, Xin Zhang, Dacheng Yang, "Downlink resource allocation for Device-to-Device communication underlaying cellular networks", IEEE 23rd International Symposium on Personal Indoor and Mobile Radio Communications (PIMRC), 2012.

[Chen13] Chen T. and Rikkinen K., "Asymmetric D2D Communication", US Patent Application No. 20130184024 A1, 2013.

[Chen14] Chen, T. and Kunnari, E., "Method and apparatus for facilitating packet scheduling for a hybrid communication network", US Pat. No. 8,675,580 issued on March 18, 2014.

[Cun03] R. Cuny, A. Lakaniemi, "VoIP in 3G Networks: An end-to-end quality of service analysis," IEEE, VTC spring, vol. 2, pp. 930--934, April 2003.

[Dop09] K. Doppler, M. Rinne, C. Wijting, C. Ribeiro, and K. Hugl, “Device-to-device communication as an underlay to LTE-Advanced networks," IEEE Commun. Mag., vol. 47, no. 12, pp. 42-49, Dec. 2009. 
[Ewa14] Ewaldo Zihan, Kae Won Choi, "Random access protocol for collision avoidance in cellular device-to-device communication", IEEE International Conference on Communications (ICC), 2014

[Fan07] Jianke Fan, Tao Chen, "Packet Scheduling Algorithms for Mixed Traffic Flow over HSDPA", PIMRC 2007

[G3003] 3GPP TR 30.03U, "Selection procedures for the choice of radio transmission technologies of the UMTS", version 3.2.0, 1998

[Gre10] GregerBlennerud,"Mobile broadband - busting the myth of the scissor effect", Ericsson Business Review, 2010

[Gu11] Jaheon Gu, Sueng Jae Bae, Bum-Gon Choi, Min Young Chung, "Dynamic power control mechanism for interference coordination of device-to-device communication in cellular networks", 3rd International Conference on Ubiquitous and Future Networks (ICUFN), 2011

[Hak10] S. Hakola, T. Chen, J. Lehtomäki and T. Koskela, "Device-toDevice (D2D) Communication in Cellular Network - Performance Analysis of Optimum and Practical Communication Mode Selection", Proceedings of IEEE Wireless Communications and Networking Conference 2010 (WCNC'10), Sydney, Australia, 2010.

[Han12] Tao Han, Rui Yin, Yanfang Xu, Guanding Yu, “Uplink channel reusing selection optimization for Device-to-Device communication underlaying cellular networks", IEEE 23rd International Symposium on Personal Indoor and Mobile Radio Communications (PIMRC), 2012

[Hol06a] H. Holma, M. Kuusela, E. Malkamäki, K. Ranta-aho, T. Chen, "VoIP over HSPA with 3GPP Release 7", PIMRC 2006, Helsinki.

[Hol06b] H. Holma, A. Toskala, "HSDPA/HSUPA for UMTS”, John Wiley, 2006.

[Hol11] Harri Holma, Antti Toskala, “LTE for UMTS: Evolution to LTEAdvanced", John Wiley, 2011.

[Hsi02] H.-Y. Hsieh and R. Sivakumar, "On Using the Ad-hoc Network Model in Cellular Packet Data Networks", ACM MOBIHOC, 2002.

[Hsi04] H.-Y. Hsieh and R. Sivakumar, "On using peer-to-peer communication in cellular wireless data networks," IEEE Trans. Mobile Comput., vol. 3, no. 1, pp. 57-72, Jan. 2004.

[IG114] ITU-T Recommendation G.114, “One way transmission time” 
[Jal00] A. Jalali et al., "Data throughput of CDMA-HDR a high efficiencyhigh data rate personal communication wireless system," in Proceedings of IEEE Vehicular Technology Conference, 2000

[Jan09] P. Janis, C.-H. Yu, K. Doppler, C. Ribeiro, C. Wijting, K. Hugl, O. Tirkkonen, and V. Koivunen, "Device-to-device communication underlaying cellular communications systems," Int. J. Commun., Netw. Syst. Sci., vol. 2, no. 3, pp. 169-178, Jun. 2009.

[Jia07] Jianke Fan, Tao Chen, "Packet Scheduling Algorithms for Mixed Traffic Flow over HSDPA", PIMRC 2007

[Kau08] B. Kaufman and B. Aazhang, "Cellular Networks with an Overlaid Device to Device Network", Proceedings of 42nd Asilomar Conference on Signals, Systems and Computers, pp. 1537-1541, Oct. 2008.

[Kos10] T. Koskela, S. Hakola, T. Chen and J. Lehtomäki, “Clustering Concept using Device-to-Device Communication in Cellular System", Proceedings of IEEE Wireless Communications and Networking Conference 2010 (WCNC'10), Sydney, Australia, 2010.

[Lap08] Lap Kong Law, Srikanth V. Krishnamurthy, and Michalis Faloutsos, "Capacity of Hybrid Cellular-Ad hoc Data Networks", INFOCOM 2008.

[Lee11] Jungha Lee, Jaheon Gu, Sueng Jae Bae, Min Chung, “A session setup mechanism based on selective scanning for device-to-device communication in cellular networks", 17th Asia-Pacific Conference on Communications (APCC), 2011.

[Lee12] Yang-Han Lee, Hsien-Wei Tseng, Chih-Yuan Lo, Yih-Guang Jan, Le-Pong Chin, Ting-Chen Song, Heng-Iang Hsu, "Using Genetic Algorithm with frequency hopping in device to device communication (D2DC) interference mitigation", International Symposium on Intelligent Signal Processing and Communications Systems (ISPACS), 2012

[Leh08] J. Lehtomäki, I. Suliman, J. Vartiainen, M. Bennis, A. Taparugssanagorn, and $\mathrm{K}$. Umebayashi, "Direct communication between terminals in infrastructure based networks," in Proc. ICT Mobile and Wireless Commun. Summit, Stockholm, Sweden, Jun. 2008.

[Li14] Yong Li, Zhaocheng Wang, Depeng Jin, Sheng Chen, “Optimal Mobile Content Downloading in Device-to-Device Communication Underlaying Cellular Networks", IEEE Transactions on Wireless Communications, 2014 
[Mcl05] S. McLaughlin and H. Haas, "Cellular communications system", Patent Application WO060182, 2005.

[Mul05] Muller, A., Tao Chen, "HSDPA Link Adaptation by Considering the Age of Channel Quality Feedback Information", VTC Fall 2005

[Mul05] Muller, A., Tao Chen, "HSDPA Link Adaptation by Considering the Age of Channel Quality Feedback Information", VTC Fall 2005

[Pah14] Pahlevani, P., Hundeboll, M., Pedersen, M.V., Lucani, D., Charaf, H., Fitzek, F.H.P., Bagheri, H., Katz, M., "Novel concepts for device-to-device communication using network coding", IEEE Communications Magazine, 2014

[Pet07] Petteri Lundén, Markku Kuusela, "Enhancing Performance of VoIP over HSDPA", VTC 2007 spring.

[Ric94] W.Richard Stevens, "TCP/IP Illustrated,Volume 1:The Protocols", Addison-Wesley, 1994, ISBN 0-201-63346-9

[San14] Sanghoon K. and Stark, W., "Full duplex device to device communication in cellular networks", International Conference on Computing, Networking and Communications (ICNC), 2014

[Song14] Lingyang Song, Niyato, D., Zhu Han, Hossain, E., “Gametheoretic resource allocation methods for device-to-device communication", IEEE Wireless Communications, 2014

[Vas13] Vasudevan, S., Sivanesan, K., Kanugovi, S., Zou, J., “Enabling Data Offload and Proximity Services Using Device to Device Communication over Licensed Cellular Spectrum with Infrastructure Control", IEEE 78th Vehicular Technology Conference (VTC Fall), 2013

[Wang11] Bin Wang, Li Chen, Xiaohang Chen, Xin Zhang, Dacheng Yang, "Resource Allocation Optimization for Device-to-Device Communication Underlaying Cellular Networks", IEEE 73rd Vehicular Technology Conference (VTC Spring), 2011.

[Wen12] Si Wen, Xiaoyue Zhu, Zhesheng Lin, Xin Zhang, Dacheng Yang, "Optimization of interference coordination schemes in Device-toDevice(D2D) communication", 7th International ICST Conference on Communications and Networking in China (CHINACOM), 2012

[Wen13] Si Wen, Xiaoyue Zhu, Xin Zhang, Dacheng Yang, "QoS-aware mode selection and resource allocation scheme for Device-toDevice (D2D) communication in cellular networks", IEEE International Conference on Communications Workshops (ICC), 2013 
[Xu10] Shaoyi Xu, Haiming Wang, Tao Chen, Qing Huang, Tao Peng, "Effective Interference Cancellation Scheme for Device-to-Device Communication Underlaying Cellular Networks", VTC Fall 2010

[Xu12] Yanfang Xu, Rui Yin, Tao Han, Guanding Yu, “Interference-aware channel allocation for Device-to-Device communication underlaying cellular networks", IEEE 1st International Conference on Communications in China (ICCC), 2012

[Xu12a] Shaoyi Xu, Jing Han, Tao Chen, "Enhanced Inter-Cell Interference Coordination in Heterogeneous Networks for LTE-Advanced", VTC Spring 2012.

[Xu12b] Shaoyi Xu, Haiming Wang, Tao Chen, “Effective Interference Cancellation Mechanisms for D2D Communication in Multi-Cell Cellular Networks", VTC Spring 2012.

[Ye14] Ye, Q., Shalash, M., Caramanis, C. and Andrews, J.G., "Resource Optimization in Device-to-Device Cellular Systems Using TimeFrequency Hopping", IEEE transaction on Wireless Communications, 2014

[Zhou13] Wei Zhou, Xiaodong Sun, Chuan Ma, Jianting Yue, Hui Yu, Hanwen Luo, "An interference coordination mechanism based on resource allocation for network controlled Device-to-Device communication", IEEE/CIC International Conference on Communications, China, 2013 


\title{
ORIGINAL PAPERS
}

\section{I \\ UPLINK CAPACITY OF VOIP ON HSUPA}

\author{
by \\ Chen, T., Kuusela, M. and Malkamäki, E. 2006 \\ Proceedings of the 63rd IEEE Vehicular Technology Conference (VTC'06-spring)
}

Reproduced with kind permission by IEEE. 


\section{Uplink Capacity of VoIP on HSUPA}

\author{
Tao Chen \\ Nokia Research Center \\ Nokia House 1, No.11, He Ping Li \\ Dong Jie \\ 100013, Beijing, P.R.China \\ tao.t.chen@nokia.com
}

\author{
Markku Kuusela \\ Nokia Research Center \\ P.O.Box 45, FIN-00045 Nokia \\ Group \\ Helsinki, Finland
}

Esa Malkamäki

Nokia Research Center P.O.Box 45, FIN-00045 Nokia Group

Helsinki, Finland

esa.malkamaki@nokia.com

\begin{abstract}
In this paper, the system performance of VoIP on HSUPA, which is investigated by the semi-analytical predictio and the system level simulations, was studied and presented. Th semi-analytical results show the capacity gain of VoIP over HSUPA than 12.2kbps on DCH in Rel'99. Further, the aforementioned prediction shows the consistency with system level simulation results in principle. Otherwise, the uplink capacity of VoIP over HSUPA with non-scheduled algorithm is mainly dependent on the delay budget and availability of the UE pow, which is indicated by the results. HARQ and power, handover can be expected to lower the required UE power an thus incer sensitivity of VolP service, a compromise between the maximum number of HARQ retransmissions and the delay budget can be considered optimize the capacity of VoIP on HSUPA.
\end{abstract}

Keywords- VoIP, HSUPA, capacity

\section{INTRODUCTION}

High-speed Uplink Packet Access (HSUPA), which is included in 3GPP Release 6 specification, has been designed to increase the uplink packet data throughput by means of Hybrid Automatic Repeat request (HARQ), shorter frame size and fas scheduling controlled by the base station. High Speed Downlink Packet Access (HSDPA) is the downlink counterpart of HSUPA in 3GPP Rel'05. Recently, there has been an increasing interest to use HSDPA/HSUPA for real-time (RT) packet switched (PS) services such as Voice Over IP (VoIP). In $3 \mathrm{GPP}$ VoIP is studied under two work items: "Continuous connectivity for packet data users" and "RT HSPA Improvement". The reasoning behind the increased interest towards VoIP is to use VoIP in All-IP network instead of using circuit switched (CS) speech, which will imply cost savings for operators as CS related part of the core network would not be needed anymore. At the same time, better capacity or coverage with VoIP can be expected, as we shall show in this paper. The challenge for the VoIP on HSDPA/HSUPA will be the delay requirement for the voice traffic. Our focus in this paper is the VoIP performance on HSUPA. We shall provide both semianalytical prediction and system simulation results for the capacity of VoIP on HSUPA, which shall show that VoIP on Rel'06 HSUPA achieves better capacity than Rel'99 CS Voice.

The rest of this paper is organized as follows. In Section II, the VoIP over HSUPA concept, and the non-scheduling for VoIP are described. Next, semi-analytical predictions for VoIP on Rel'99 and HSUPA are shown. Further, the system simulation methodology is explained in Section IV and results are presented and discussed in Section V. And finally, conclusions are drawn in Section VI.

\section{CONCEPT OF VOIP OVER HSUPA}

Here, the VoIP over HSUPA is described, the VoIP traffic modeling and scheduling algorithm used in this study are presented. The criteria are defined to evaluate the performance of VoIP on HSUPA. HSUPA is described, e.g., in [1]

\section{A. VoIP over HSUPA}

VoIP over HSUPA can be implemented in several different ways. The HSUPA specification defines two TTI lengths for EDCH: $10 \mathrm{~ms}$ and $2 \mathrm{~ms} .10 \mathrm{~ms}$ TTI is mandatory for all UEs and support of $2 \mathrm{~ms}$ TTI depends on the UE capability. Furthermore, two different scheduling modes are defined for HSUPA: Node B scheduling mode with L1/MAC control signaling in uplink and downlink, and RNC controlled nonscheduled mode.

For $10 \mathrm{~ms}$ TTI, 4 HARQ processes are specified which implies a round trip time of $40 \mathrm{~ms}$ for the fast HARQ. Thus only one retransmission is possible in order to keep the transmission delay below $80 \mathrm{~ms}$. Figure 1 illustrates the transmission of VoIP packets over E-DCH. A new VoIP packet is received from the speech codec every $20 \mathrm{~ms}$. Thus every second TTI is occupied with a new VoIP transmission. If the retransmission is needed, then the transmission of the next VoIP packet is delayed by $10 \mathrm{~ms}$ and worst-case transmission delay increases to $60 \mathrm{~ms}$.

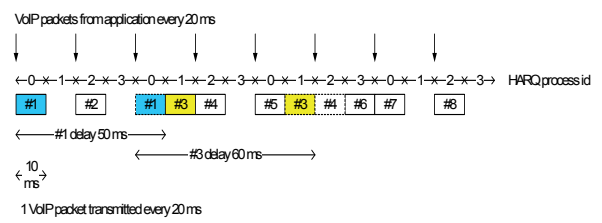

Figure 1. VoIP on E-DCH with $10 \mathrm{~ms}$ TTI

For $2 \mathrm{~ms}$ TTI, 8 HARQ processes are specified and the HARQ round trip time is $16 \mathrm{~ms}$. The $80 \mathrm{~ms}$ transmission delay limit allows using up to 4 transmissions. Figure 2 illustrates 
VoIP transmission with a maximum of 3 retransmissions resulting in a worst-case delay of $50 \mathrm{~ms}$. For $2 \mathrm{~ms}$ TTI, it is possible to limit the number of HARQ processes used by one VoIP user. This can be used for time multiplexing of differen users into separate HARQ processes. However, if 3 retransmissions per packet and no extra delay due to HARQ process allocation are allowed, then 4 HARQ processes out of 8 needs to be allocated for each user.

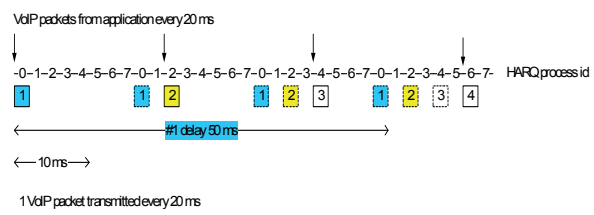

Figure 2. VoIP on HSUPA with 2ms TTI

The advantage of $10 \mathrm{~ms}$ TTI is that all UEs support it. It requires lower peak rates and performs better at cell edge and in soft handover than $2 \mathrm{~ms}$ TTI. With $2 \mathrm{~ms}$ TTI a somewhat higher cell capacity is achievable since more HARQ retransmissions can be allowed. Also time multiplexing of different users is possible.

B. Scheduling for VoIP traffic

The non-scheduled transmission of E-DCH is specified for guaranteed bit rate services and is therefore suitable for VoIP. The maximum number of bits per MAC-e PDU per MAC-d flow is configured by SRNC through RRC signaling. The allowed bit rate should take into account the speech codec rate, the header compression efficiency and variations, and the the header compression efficiency and variations, and the
existence of Real time control protocol (RTCP) packets. The non-scheduled data rate can be changed via RRC signaling.

The Node B scheduling with uplink rate requests and downlink rate grants is also possible for VoIP. Node B can send an absolute grant to the UE and activate only some HARQ processes (for $2 \mathrm{~ms} \mathrm{TTI}$ ). This enables time multiplexing of users. The advantage of scheduled transmission is that the UE can request higher grant if needed, for instance, due to RTCP packets. The scheduled grant is, however, power allocation that does not guarantee a minimum bit rate and in soft handover other non-serving Node Bs can lower the serving grant of the UE. Therefore, RNC controlled nonscheduled transmission is more attractive for VoIP service.

\section{Delay requirement}

According to [2], the maximum acceptable mouth-to-ea delay for voice is on the order of $250 \mathrm{~ms}$. If the delay for core network, RNC processing and Iub transport is approximately $100 \mathrm{~ms}$, the tolerable delay for MAC-hs buffering/scheduling , transmission and detection is less than $80 \mathrm{~ms}$. Here it is assumed that both end-users are HSPA users.
III. SEMI-ANALYTICAL StUdy

In the semi-analytical study, we derive the cell Noise Rise for the VoIP on HSUPA by using the following typical uplink load formula (see, e.g., [3]):

$$
N R_{d B}=-10 \log 10\left(1-\frac{E b N o}{W / R} N \cdot v \cdot(1+i)\right)
$$

Where $N$ is the number of users per cell, $v$ is the average activity factor, EbNo is signal energy per bit divided by noise spectral density, $W$ is the chip rate, $R$ is the data rate, $i$ is the other cell to own cell interference ratio, and $N R_{d B}$ is the noise rise with $\mathrm{dB}$. Based on the equation, we can predict the uplink capacity of $12.2 \mathrm{kbps}$ on DCH for R99 as the reference case.

For VoIP on HSUPA, E-DPCCH and E-DPDCH are activated only during the data transmission. Hence, we can further express the above equation as below.

$N R_{d B}=-10 \log 10\left(1-\left(E c N Q_{c} \cdot v_{c}+E c N Q_{e} \cdot v_{e}\right) \cdot N \cdot(1+i)\right)$

Where $E c N o$ is for DPCCH, EcNo for E-DCH (including E-DPCCH and E-DPDCH), $v_{c}$ activity factor for DPCCH, $v_{e}$ activity factor for E-DCH

$v_{e}$ can be derived from the voice activity factor $\left(v_{i}\right)$, average transmission times of HARQ (AvgTxs) and the E-DCH activity factor $\left(v_{d}\right)$, i.e.,

$$
v_{e}=v_{i} \cdot \operatorname{AvgTxs} \cdot v_{d}
$$

$\mathrm{ECNo}_{c}$ and $\mathrm{EcNo}_{e}$ are from the link level simulation including HARQ. And $v_{c}$ will be 1.0 with the continuous DPCCH transmission.

Both results of $12.2 \mathrm{kbps}$ on DCH and VoIP on HSUPA are shown in Figure 3 assuming $i=0.65$ and an EbNo requirement of $4 \mathrm{~dB}$ for $12.2 \mathrm{kbps}$ on $\mathrm{DCH}$

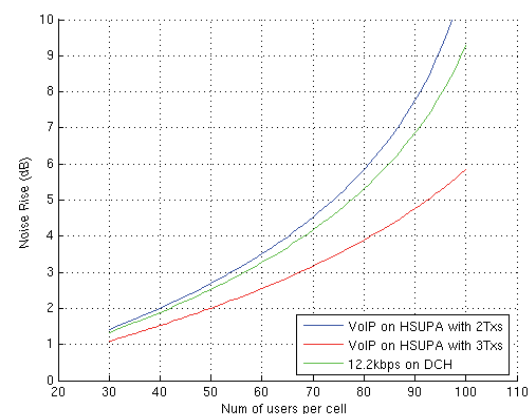

Figure 3. Semi-analytical predication of Noise Rise. 
Compared with Rel'99, about $18 \%$ uplink capacity gain corresponding $6 \mathrm{~dB}$ noise rise is observed for VoIP on HSUPA with 3 transmissions in HARQ from semi-analytical results. In reality, the gain can be more due to the limitation of the capacity in the downlink for Rel'99 normally.

Besides, more transmissions in HARQ will lower the noise rise with a kind of time diversity. But at the same time, it will result in a large packet delay. Therefore, a compromise between the number of retransmission in $\mathrm{L} 1$ and the delay budget can be needed for VoIP on HSUPA.

\section{Modelling And Assumptions}

In this section, modelings of key features related to VoIP are depicted, which include VoIP traffic modeling, and physical channel modeling affecting the capacity. Capacity and outage definitions are also described as the criteria of Qos for VoIP. For the simulation environment, a quasi-static system level simulator and assumptions are introduced.

A. VoIP traffic modeling

The duration of each VoIP call is sampled from a negative exponential distribution function with an average call length of 60 seconds. Discontinuous transmission (DTX) is simulated by $50 \%$ probability of transmission and silent periods, i.e., on and off periods. The duration of both on and off periods are negative exponentially distributed with an average of 3 negative exponentially distributed with an average of 3
seconds. During on periods, a voice encoder generates a seconds. During on periods, a voice encoder generates a
payload per VoIP packet of 31 bytes every $20 \mathrm{~ms}$, payload per VoIP packet of 31 bytes every $20 \mathrm{~ms}$, And another 9 bytes from the headers of RTP/UDP/IP/PDCP/RLC with header compression will be included in each VoIP packet. In our simulation model, VoIP packets are assumed to arrive at the UE every $20 \mathrm{~ms}$ during on periods.

B. Channel modelling for HSUPA

In our simulations, three types of uplink dedicated physical channels, the uplink Dedicated Physical Control Channel (uplink DPCCH), the uplink E-DCH Dedicated Physical Data Channel (uplink E-DPDCH), the uplink E-DCH Dedicated Physical Control Channel (uplink E-DPCCH), will be utilized for VoIP traffic on HSUPA. The DPCCH, the E-DPCCH and the E-DPDCH are I/Q code multiplexed [4]

The DPCCH will carry control information generated at Layer 1, which consists of known pilot bits to support channel estimation for coherent detection, and transmit power-control (TPC) commands. Optional feedback information (FBI), and an optional transport-format combination indicator (TFCI) are not assumed here. DPCCH will be transmitted all the time.

One E-DPCCH on each radio link is modeled to transmit control information associated with the E-DCH as the overhead. Similar to DPCCH, the control information carried by E-DPCCH will not be combined by L1 HARQ. Therefore, the decoding of E-DPCCH for each transmission will require a specific $\beta_{e c}$ [5], which ensures that E-DPCCH transmission is error free with the power level corresponding to HARQ transmissions. Here, the power ratio $\left(\beta_{c c} / \beta_{c}\right)^{2}$ with $3 \mathrm{~dB}$ is used for maximum 4 transmissions based on the link level simulation study.

One E-DPDCH on each radio link is modeled to carry the E-DCH transport channel. The data transmitted by E-DPDCH will experience the L1 HARQ chase combining in the NodeB side. And L1 HARQ with chase combining is modeled based on [6]. The power ratio $\left(\beta_{e d} / \beta_{c}\right)^{2}$ with $8 \mathrm{~dB}$ is used as an optimized value according to the link level simulation study.

Therefore, the total transmission power for UE with VoIP traffic can be expressed by equation (4).

$$
P_{\text {Total }}=P_{D P C C H} \cdot\left(1+\left(\frac{\beta_{e c}}{\beta_{c}}\right)^{2}+\left(\frac{\beta_{e d}}{\beta_{c}}\right)^{2}\right)
$$

The E-DPDCH and E-DPCCH are always frame aligned with each other. Each radio frame with a frame length of $10 \mathrm{~ms}$ is divided in 5 subframes, each of length $2 \mathrm{~ms}$. In the level simulation, $2 \mathrm{~ms}$ TTI is adopted for the study using the subframe structure.

\section{Criteria of Qos for VoIP}

The outage is introduced to evaluate the performance of VoIP on HSUPA. Here, an outage is counted if $5 \%$ of the VoIP packets for one user are not received within the delay budget i.e., $80 \mathrm{~ms}$ (when monitored over a 10 seconds on period).

Each newly arrived VoIP packet in the UE is associated with a discard timer. Whenever a buffered packet has been transmitted, it is moved to the Hybrid-ARQ manager and its discard timer is deactivated. Hence, whenever a packet has been transmitted, it can be dropped only if it has not been successfully received after the maximum number of allowed transmissions.

Further, the capacity is defined as the maximum number of VoIP users per cell, which can be supported without exceeding a $5 \%$ cell outage level.

\section{Environment}

A quasi-static system level simulator where all necessary RRM algorithms as well as their interactions are modeled is used to investigate the performance of VoIP on HSUPA. Thi tool includes a detailed simulation of the users within multiple cells. The fast fading is explicitly modeled for each use according to the ITU Vehicular-A profile. Otherwise, from the methodology point view, the quasi-static simulator is based on descriptions in [7]. A wrap-around multi-cell layout modeling several layers of interference is utilized in this study.

Main parameters used in the system simulation are summarized in Table I

TABle I. Parameters Settings in the SimUlation

\begin{tabular}{|l|l|}
\hline \multicolumn{1}{|c|}{ Parameter } & \multicolumn{1}{c|}{ Value } \\
\hline Frame Size & $2 \mathrm{~ms}$ TTI \\
\hline Inter site distance & $2.8 \mathrm{~km}$ \\
\hline Cell configuration & ITU Veh-A, Macrocell \\
\hline Voice call mean length & 60 seconds \\
\hline
\end{tabular}




\begin{tabular}{|l|l|}
\hline \multicolumn{1}{|c|}{ Parameter } & \multicolumn{1}{c|}{ Value } \\
\hline Voice on/off mean length & 3 seconds \\
\hline Payload size & 31 bytes \\
\hline Compressed header size & 9 bytes \\
\hline UE speed & $3 \mathrm{kmph}$ \\
\hline Voice Activity & 0.5 \\
\hline Number of HARQ channels & 8 \\
\hline Max number of L1 transmissions & 4 \\
\hline$\left(\beta_{e c} / \beta_{c}\right)^{2}$ & $3 \mathrm{~dB}$ \\
\hline$\left(\beta_{e d} / \beta_{c}\right)^{2}$ & $8 \mathrm{~dB}$ \\
\hline Scheduling algorithm & Non-scheduled \\
\hline WinAdd for soft handover & $1 / 4 \mathrm{~dB}$ \\
\hline Discarded timer & $80 \mathrm{~ms}$ \\
\hline Delay budget & $80 \mathrm{~ms}$ \\
\hline Residual FER Target & $1 \%$ \\
\hline Outage observation window length & $10 \mathrm{~seconds}$ \\
\hline Cell outage threshold & $5 \%$ \\
\hline &
\end{tabular}

V. System LeVel Simulation Results

In this section, some simulation results are shown to confirm semi-analytical results and analysis. And more details, which are not available from semi-analytical study, are presented to provide a comprehensive analysis.

In Figure 4, we can see a consistency between semianalytical predication and simulation results in principle. With higher UE number, more deviations between them are observed due to the limited UE power in the simulations, which can conflict with the semi-analytical prediction. In the semi-analytical study, a premise is that EbNo will be kept very well for each UE, which requires an ideal power control and enough UE power. However, in the real world simulated by the simulator, due to non-scheduled algorithm for VoIP traffic, the noise rise will not be controlled. Furthermore, higher noise rise will rise up the required UE power to maintain the EbNo. Finally, higher interference with more UEs will result in the coverage shrinking effect due to the power shortage. Then less UEs with a specific EbNo can be satisfied in the system.



Figure 4. Comparison of simulation results and semi-analytical predication.
Corresponding to $6 \mathrm{~dB}$ Noise Rise normally used as the load target with $75 \%$ load in the uplink, the UE number per cell is about 85 with $1 \mathrm{~dB}$ WinAdd and 93 with $4 \mathrm{~dB}$ WinAdd.

In Table II, the capacity and HSUPA gain over Rel'99 are summarized. Obviously, even compared with the theoretical value of Rel'99 corresponding 6dB noise rise, HSUPA with VoIP can provide up to $9.4 \%$ gain

TABLE II. UPLINK CAPACITY GAING OVER REL'99

\begin{tabular}{|l|l|l|l|}
\hline & \multicolumn{1}{|c|}{ Rel'99 } & $\begin{array}{c}\text { HSUPA } \\
\text { (1dB WinAdd) }\end{array}$ & $\begin{array}{c}\text { HSUPA } \\
\text { (4dB WinAdd) }\end{array}$ \\
\hline UEs/Cell & 85 & 85 & 93 \\
\hline Gain & $0 \%$ & $0 \%$ & $9.4 \%$ \\
\hline
\end{tabular}

On the other hand, the gain is dependent on many factors, such as WinAdd, the maximum allowed transmission number, beta factors for E-DPCCH and E-DPDCH, the header size, the adopted data rate. All of them are more or less related with the UE power. Among them, the maximum transmission number will affect the packet delay as well.

In Figure 5, the capacity from cell outage-wise is shown. I also matches with the result of noise rise well, i.e. they are correlated. With higher noise rise, UE in the cell edge will not follow the SIR target any more due to the shortage of the power. Then, the un-ideal power control can lead to the increase of the packet error ratio. Hence, the cell outage will increase since more packets can't be received correctly within the delay budget.

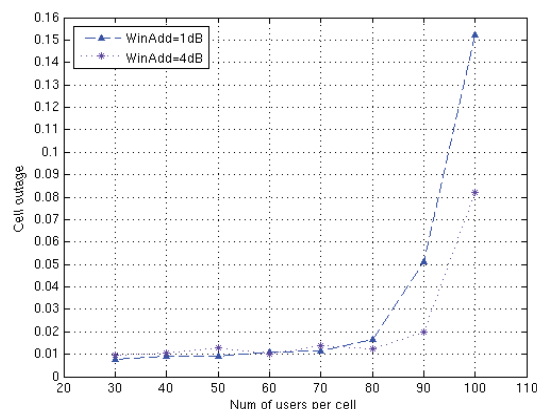

Figure 5. Cell outage with different WinAdd for soft handover.

Soft handover will benefit to increase the uplink capacity for VoIP on HSUPA by lowering the required the UE power. More capacity is shown with $4 \mathrm{~dB}$ WinAdd when compared with $1 \mathrm{~dB}$.

Figure 6 shows the power distributions, which verifies that the uplink capacity of VoIP on HSUPA with non-scheduled algorithm depends on the available UE power against the noise rise. 
In HSDPA, the packet scheduling will impact the performance due to the channel sharing. And the packet delay will be one of the main factors contributing to the outage, i.e. capacity.

Different with HSDPA, there will be the dedicated uplink physical channel for each UE in HSUPA. Non-scheduled algorithm is utilized to reduce the packet delay with the cost of high interference. In this situation, if the HARQ transmission delay can be within the delay budget, for example, $80 \mathrm{~ms}$, the packet delay will not be a problem. Instead, the available UE power will be the bottleneck for the capacity. Besides, due to the delay requirement, non-scheduled algorithm will be expected to have requirement, non-scheduled algorithm will be expected to have
more gain than Node-B scheduling since the scheduling delay more gain than
can be avoided.

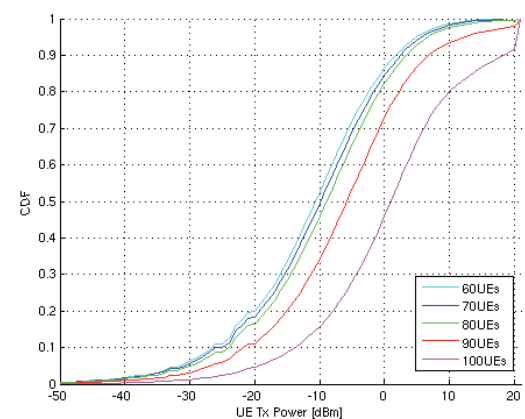

Figure 6. UE power cumulative distributions with different Ues/cell
VI CONCLUSIONS

We have presented the system performance of VoIP on HSUPA, which is investigated by the semi-analytical prediction and the system level simulations. With the semianalytical results, the gain is observed clearly when compared with $12.2 \mathrm{kbps}$ on DCH in Rel'99. Further, with $12.2 \mathrm{kbps}$ on DCH in Rel'99. Further,
the aforementioned prediction shows the consistency with the aforementioned prediction shows the consistency with
system level simulation results in principle. On the other hand, the uplink capacity of VoIP on HSUPA with non-scheduling is dependent on the delay budget and availability of the UE power, which is indicated by the results. HARQ and soft handover can benefit to lower the required UE power and the increver and thus increase the capacity. A compromise between the maximum number of HARQ retransmissions and the delay budget can be considered to optimize the capacity of VoIP on HSUPA.

\section{ACKNOWLEDGMENT}

We would like to thank Karri Ranta-Aho, working at Nokia Networks for providing the help and guide in this study.

\section{REFERENCES}

[1] 3GPP TS 25.309, "FDD Enhanced Uplink; Overall description; Stage 2"

[2] ITU-T Recommendation G.114, "One way transmission time"

[3] H. Holma, A. Toskala (Editors), "WCDMA for UMTS - Radio Acces This

[4] 3GPP TS 25.211, "Phisical channels and mapping of transport channel channels (FDD)

[5] 3GPP TS 25.214, "Physical layer procedures (FDD)"

[6] F. Frederiksen, T.E. Kolding, "Performance and Modelling of WCDMA/HSDPA transmission/H-ARQ Schemes", IEEE VTC, Fall, pp.472-481, 2002.

[7] 3GPP2 Technical Specification Group C, 3GPP2/TSG-C.1002: "1X EVDV Evaluation Methodology" 


\title{
DPCCH GATING GAIN FOR VOICE OVER IP ON HSUPA
}

\author{
by \\ Fresan, O., Chen, T., Malkamäki, E. and Ristaniemi, T. 2007 \\ Proceedings of IEEE Wireless Communications and Networking Conference 2007 \\ $\left(\mathrm{WCNC}^{\prime} 07\right)$
}

Reproduced with kind permission by IEEE. 


\title{
DPCCH Gating Gain for Voice over IP on HSUPA
}

\author{
Oscar Fresan ${ }^{\dagger}$, Tao Chen ${ }^{\ddagger}$ Esa Malkamäki ${ }^{\ddagger}$, Tapani Ristaniemi ${ }^{\dagger}$
}

\author{
†Institute of Communications Engineering, \\ Tampere University of Technology \\ P.O. Box 553, FIN-33101, Tampere, Finlan \\ \{oscar.fresan, tapani.ristaniemi\} @ tut.fi
}

\begin{abstract}
In this paper, the concept of DPCCH (Dedicated Physical Control Channel) gating for HSUPA (High Speed Uplink Packet Access) is analyzed. Gating technique has recently been under consideration within WCDMA to inactivate control channels during silent periods of the users, and hence, not to misuse capacity. In order to study the concept benefit, a concrete real-time service is selected in this paper: Voice over IP (VoIP) for mobile communications. It will be shown that VoIP would be highly beneficed by DPCCH gating inclusion in 3GPP specifications. Both analytical and simulation studies were run to confirm the gain expectitions.
\end{abstract}

Keywords- DPCCH gating, VoIP, HSUPA

$$
\text { I. INTRODUCTION }
$$

High speed uplink packet access (HSUPA) concept was elaborated in the release 6 of the $3 \mathrm{GPP}$ specifications for the third mobile generation. It is the uplink counterpart of the high speed downlink channel (HSDPA) that was already introduced in release 5. Both high speed channels were conceived in order to achieve higher capacities and coverage for high transmission rates by the means of innovative techniques such as HARQ (Hybrid-ARQ), short frame size and Node B controlled packet scheduling.

Release 7 , currently under development, continues to enable even higher data rates and capacity improvements in addition to improved support for real-time services e.g. Voice over IP (VoIP). At the radio access network side, continuous connectivity is one of the enhancements. The main problem to support continuous connection in WCDMA is the high number of UE's not transmitting, while keeping the control channels in use which overwhelms the cell capacity. One of the proposals to overcome this situation is the discontinuous transmission of the DPCCH channel when the UE is in idle mode. This is referred to as the DPCCH gating technique.

Voice over IP service has been proposed thinking on the evolution of UMTS to an all-IP network. It is meant to substitute the present circuit switched voice connections, thus it should show a clear gain over them in order to be worth implementing.

\author{
${ }^{\ddagger}$ Nokia Research Center \\ P.O. Box 45, FIN-00045 \\ Nokia Group Helsinki, Finland \\ \{tao.t.chen, esa.malkamaki\}@ nokia.com
}

As one of the real-time services considered in the continuous connectivity work item, VoIP would be highly beneficed by the gating technique inclusion. What is more, the characteristics of gating technique inclusion. What is more, the characteristics of
VoIP service makes it easier to implement the gating process as we will explain later.

The paper is organized as follows: in section II, the DPCCH gating technique is described. Section III details the VoIP concept. Section IV covers the analytical study previous to the simulation process. Through section $\mathrm{V}$ the modeling and assumptions considered in the simulator are presented. The assumptions considered in the simulator are presented. The
actual simulation results are analyzed in section VI and final actual simulation results are analyzed
conclusions are drawn in Section VII.

\section{DPCCH GATING CONCEPT}

Previously to release 7 (figure 1), DPCCH channel was transmitted continuously regardless whether there is actual user data to be transmitted or not, thus highly loading the cell. An ideal solution for increasing the uplink capacity would be to keep the UE silent during the periods that is not transmitting any data and activate the control channels just for the transmissions periods. Therefore, the interference to other users would be reduced. This is the basic idea behind the DPCCH gating concept presented in [1] and depicted in figure 2 .

Due to DPCCH gating, it might be difficult for the network to distinguish an inactive period from a loose connection. That is why the DPCCH transmission is desirable to not be totally silent but transmitted periodically following a predefined pattern.

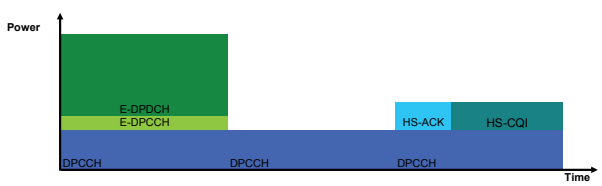

Figure 1. HSUPA transmission in Rel'6 


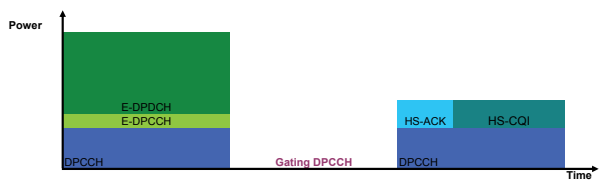

Figure 2. HSUPA transmission with gating DPCCH

Another issue to consider is the transmission power. After gating period without tracking the received SIR, the channe response variations could degrade the transmission and increase the interference between the UEs. In the 3GPP specifications for DPCCH gating [1], optional power control preambles are defined to be sent previous to the data channel reactivation. It is interesting to point out here that the HARQ protocol specified interesting to point out here that the HARQ protocol specified for HSUPA helps to decrease the impact of inaccuracies in the
power control algorithm, as it was illustrated in [2], and so it will reduce the gating technique on the power control performance.

\section{VOIP OVER HSUPA}

A. VoIP packet delay requirements

VoIP is defined as a conversational class service and thus the packet delay should be maintained strictly under som reasonable limits. According to [3], the maximum mouth-to-ear delay acceptable for a good quality communication should be lower than $250 \mathrm{~ms}$. Supposing that the network delays, such as core network, RNC processing and Iub transport is approximately $100 \mathrm{~ms}$, there is a $150 \mathrm{~ms}$ margin for the ai interface delays. Supposing also that both UE's are communicating through HSDPA/HSUPA channels, then the maximum acceptable delay of one way transmission must be under a $80 \mathrm{~ms}$ delay budget.

VoIP follows a predictable pattern of data packets and silence periods, which are generated by the Adaptive Multi-Rate (AMR) speech codec. In our study we suppose that the AMR speech codec produces one packet every $20 \mathrm{~ms}$, compounded of 320 information bits, during the activity periods.

B. VoIP trasnmission over E-DCH with $H A R Q$

Two possible TTI sizes have been specified for HSUPA: $2 \mathrm{~ms}$ and $10 \mathrm{~ms}$. Every third generation-compatible user's equipmen must support $10 \mathrm{~ms}$ TTI while $2 \mathrm{~ms}$ is optional. $2 \mathrm{~ms}$ will transmit at higher peak rates, with lower delays, but requiring higher transmission power from the UE than $10 \mathrm{~ms}$ TTI and therefore performing worst in the cell edge and soft handover situations.

The synchronous HARQ protocol is used in HSUPA, i.e., retransmissions have to take place at a fixed duration after the previous transmission). For the $10 \mathrm{~ms}$ TTI frame size, 4 parallel processes are specified in [4], thus a packet retransmission would be sent $40 \mathrm{~ms}$ after the first transmission. Therefore, the maximum allowed transmission number would be two, since a third retransmission would imply packet delays beyond the $80 \mathrm{~ms}$ delay budget. The transmission process is sketched in the following figure. As it can be seen, the retransmission of packet $\# 1$ implies an extra delay for packet \#3, setting the maximum packet delay to $60 \mathrm{~ms}$



Figure 3 VoIP over HSUPA with 10ms TTI size

For a $2 \mathrm{~ms}$ TTI size, [4] defines the HARQ protocol with 8 parallel processes, delaying a retransmission by $16 \mathrm{~ms}$ from the first transmission. In this case, up to 4 transmissions could be contemplated without exceeding the $80 \mathrm{~ms}$ margin. Figure 4 shows a possible transmission process with a maximum of 3 transmissions

VolP peckes from applicationevery $20 \mathrm{~m}$

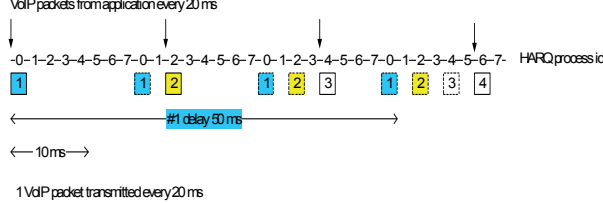

Figure 4 VoIP over HSUPA with $2 \mathrm{~ms}$ TTI size

C. Scheduling scheme

Two scheduling schemes are defined for HSUPA: Node B controlled scheduling transmission and RNC controlled nonscheduling transmission. However, the former scheme does not guarantee a minimum bit rate since the scheduling algorithm decides whether to allocate any power to the user or not. On the decides whether to allocate any power to the user or not. On the
other hand non-scheduled transmission defines a minimum data rate that the UE can transmit at without any previous request. Therefore, NRC controlled non-scheduling transmission is the most suitable choice for VoIP traffic.

VoIP can be clearly beneficed by the gating technique since usually a mean of $50 \%$ of the time a user keeps silent during a voice conversation. Drawing on this fact, only silence descriptors (SID) are sent every $160 \mathrm{~ms}$ as soon as a silence is detected. Thus, during the user's silence periods, the UE could 
transmit discontinuously on the DPCCH. On the other hand, during the active periods, the voice coder produces one packe every $20 \mathrm{~ms}$. As the transmission is not continuous, a DPCCH gating transmission could be also considered during voice activity periods. However, it should be taken into consideration the retransmissions due to the HARQ algorithm, which would decrease the gating opportunities during activity periods as it can be seen in figure 1 .

\section{Semi-Analytical StUdy}

We should answer now to the question of how much gain we can expect from the DPCCH gating technique. We can take as initial point for the analysis the time percent that DPCCH should be on, i.e. the DPCCH activity factor that we will call $v_{c}$. From the gating concept definition, $v_{c}$ will be equivalent to know the percent of the time that data is sent in any channel dependent on $\mathrm{DPCCH}$ transmission,

$$
v_{c}=v_{e}+v_{A C K}+v_{C Q I} .
$$

Here $v_{e}$ is the HSUPA activity factor and $v_{A C K}$ and $v_{C O}$ correspond to the activity factors of HS-DPCCH and feedback channel for HSDPA, respectively, considering that HSDPA holds the VoIP downlink transmission. On the other hand, $v_{e}$ is equal to the product of the voice DTX activity factor $\left(v_{i}\right)$, the frames arrival rate to the physical layer $\left(R_{F}\right)$ and the average transmission number due to the HARQ scheme (AvgTx). That

$$
v_{e}=v_{i} \cdot R_{F} \cdot \operatorname{AvgTx} .
$$

In equation (1) we have assumed that HSUPA and HS-DPCCH never overlap in time, so the DPCCH activity equals the sum of activities. On the other hand, we are considering the ideal DPCCH gating, i.e. DPCCH is not transmitted if no dependent channel is transmitted and thus no power control preambles are in consideration. We presume that both assumptions somewhat compensate each other.

Once we got the percent of time the DPCCH will be on, we can measure the system capacity through the noise rise formula [5],

$$
N R_{d B}=-10 \log _{10}(1-E c N o \cdot N \cdot v \cdot(1+i)) .
$$

Where $N$ is the amount of UEs, $v$ is the activity factor and finally $i$ represents the interference level from other cells. EcNo is the signal to noise target per chip after the antenna combining.

Four physical channels take part in VoIP communications, each one with different $E c N o$ requirements and activity factor. Hence, we can divide the formula as

$$
\begin{aligned}
N R_{d B}= & -10 \log _{10}\left[1-\left(E c N o_{c} \cdot v_{c}+E c N o_{e} \cdot v_{e}+\right.\right. \\
& \left.\left.+E c N o_{h} \cdot v_{h}\right) \cdot N \cdot(1+i)\right]
\end{aligned} .
$$

Where $E c N o$ and $v_{c}$ correspond to DPCCH, EcNo $o_{e}$ and $v_{e}$ to HSUPA related physical channels (E-DPCCH and E-DPDCH together) and finally $E c N o_{h}$ and $v_{h}$ to HS-DPCCH. $v_{h}$ is the sum of $v_{a c k}$ and $v_{c q i}$ supposing they share the same target $E c N o_{h}$.

At this point we are able to draw the cell noise rise figure with respect to the number of UEs in the cell in figure 5. The required $E c N o$ values will be obtained from the link level simulations.

We can first focus on continuous DPCCH. It is noticeable that VoIP shows similar or worse performance than $12.2 \mathrm{Kbps}$ on DCH channels using $10 \mathrm{~ms}$ TTI size: the reduction on channe activity ( 0.67 for $12.2 \mathrm{Kbps}$ on DCH to $0.41-0.31$ for $10 \mathrm{~ms}$ ) does not compensate the worse channel condition for higher data rates needed in VoIP. 2ms TTI already improves $12.2 \mathrm{kbps}$ data rates needed in VoIP. $2 \mathrm{~ms}$ TTI already improves $12.2 \mathrm{kbps}$
on DCH thanks to a farther reduction on the activity factor down to 0.15 .

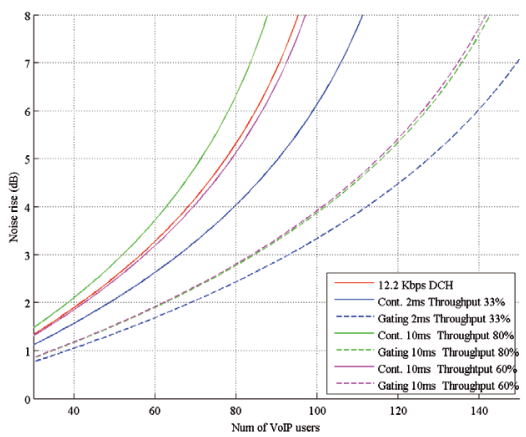

Figure 5 Semi-analytical cell noise rise for two different throughput values

The DPCCH gating technique will permit VoIP to finally beat voice $12.2 \mathrm{kbps}$ circuit switched capacities. With $10 \mathrm{~ms}$ TTI size and considering a maximum allowed noise rise of $6 \mathrm{~dB}$, we 
obtain gating gains of $50 \%$ and $64 \%$ respecting the continuou $\mathrm{DPCCH}$ simulations. In the other hand, $2 \mathrm{~ms}$ gets a $40 \%$ gatin gain. With this improvement in performance, $10 \mathrm{~ms}$ TT presents capacities around $50 \%$ higher than circuit switched transmissions, meanwhile $2 \mathrm{~ms}$ TTI goes up to $64 \%$ gain.

The throughput is defined as the inverse of the average transmission number. In continuous DPCCH transmission, a lower throughput (higher transmission number) presents lowe noise rise (see figure 5) due to the fact that lower power is needed as the received SIR for each transmission will be finally combined.

However, Gating DPCCH behaves in the opposite way with the average transmission number. In (2), a smaller AvgTx value results in lower DPCCH activity factor and therefore, longer gating periods are be possible. This is why we obtain bette gating gains: higher throughput means smaller amount of retransmissions, which results in lower DPCCH activity. Consequently we observe in figure 5 that both throughput value get the same results with the Gating DPCCH technique: there is a tradeoff between the gating DPCCH and HARQ.

\section{MOdeling AND ASSUMPTIONS}

\section{A. VoIP modelling}

The VoIP call duration is fixed to $60 \mathrm{~s}$. Considering discontinuous transmission, the 'on' and 'off' periods duration are both modeled as a decaying exponential with $3 \mathrm{~s}$ mean During the 'on' phases, VoIP packets are generated every 20 ms, containing 31 bytes from the voice codec and 9 bytes of header from layers on top of MAC 31 bytes every $20 \mathrm{~ms}$ corresponds to a source rate of $12.2 \mathrm{Kbps}$. During the 'off' periods no SID transmission is implemented.

\section{B. DPCCH gating modelling}

The gating concept has been modeled in the simplest manner. At any TTI that a packet is not received in the MAC laye $\mathrm{DPCCH}$ is not transmitted at all, i.e. no activity pattern is considered when neither HSUPA nor HS-DPCCH is transmitted. Regarding the fast power control, after a gating transmitted. Regarding the fast power control, after a gating
period the UE transmits with exactly the same power as before the off period, as DPCCH power control preamble is neither implemented.

\section{HSUPA modeling}

Three different physical channels, I/Q code multiplexed [6], are need for the uplink transmission of VoIP traffic: DPCCH, Enhanced dedicated physical control channel (E-DPCCH) and Enhanced dedicated physical data channel (E-DPDCH). The high speed downlink physical control channel (HS-DPCCH), feedback channel for HSDPA, has also been included in the study, supposing that the downlink VoIP frames are delivere through HSDPA. As pointed out in the semi-analytical study in section IV, HS-DPCCH should be contemplated for the DPCCH activity factor calculations in gating scenario.

\section{VoIP quality of service criteria}

In our study, two different criteria have been considered to measure the system capacity: the cell outage percent and the cell noise rise. The cell outage deals with the percent of discarded frames. We state that an UE is in outage situation if $5 \%$ of the frames are erroneous or discarded over a $10 \mathrm{~ms}$ period. Therefore, the system will reach the maximum allowed capacity when up to $5 \%$ of the UE in the cell are in outage.

The second criteria, the cell noise rise level, provide us a link with the semi-analytical study, as the latest is based on the received signal to noise ratio at the base station. The capacity will be given by the number of UEs in the cell when a $6 \mathrm{~dB}$ mean noise rise is measured at the base station.

\section{E. Environmen}

In order to analyze system level performance, a quasi-static simulator is used based on description in [7]. The protocol layers implemented are MAC and Physical layers. Layers above MAC are only considered in the MAC SDU size. All the needed RRM algorithms were implemented. The main simulation parameters are summarized in table 1 .

TABLE I. SIMULATION PARAMETERS SETTINGS

\begin{tabular}{|c|c|}
\hline Parameter & Value \\
\hline Frame Size & $10 \mathrm{~ms}$ and $2 \mathrm{~ms}$ TTI \\
\hline Inter site distance & $2.8 \mathrm{~km}$ \\
\hline Cell configuration & ITU Veh-A, Macrocell \\
\hline Voice call length & 60 seconds \\
\hline Voice on/off mean length & 3 seconds \\
\hline Payload size & 31 bytes \\
\hline VoIP packet arrival interval & $\begin{array}{l}20 \mathrm{~ms} \text { (with } 10 \mathrm{~ms}, 2 \text { packets } \\
\text { transmitted every } 40 \mathrm{~ms} \text { ) }\end{array}$ \\
\hline Compressed header size & 9 bytes \\
\hline UE speed & $3 \mathrm{kmh}$ \\
\hline Voice Activity & 0.5 \\
\hline Number of HARQ channels & $4(10 \mathrm{~ms}) / 8(2 \mathrm{~ms})$ \\
\hline Max number of L1 transmissions & $2(10 \mathrm{~ms}) / 4(2 \mathrm{~ms})$ \\
\hline$\left(\beta_{e c} / \beta_{c}\right)^{2}$ & $-7.96 \mathrm{~dB}(10 \mathrm{~ms}) / 3 \mathrm{~dB}(2 \mathrm{~ms})$ \\
\hline$\left(\beta_{e d} / \beta_{c}\right)^{2}$ & $8 \mathrm{~dB}$ \\
\hline Scheduling algorithm & Non-scheduled \\
\hline DPCCH gating patern & No transmission patern \\
\hline DPCCH gating power preamble length & 0 slots \\
\hline Discarded timer & $80 \mathrm{~ms}$ \\
\hline Delay budget & $80 \mathrm{~ms}$ \\
\hline Outage observation window length & 10 seconds \\
\hline Cell outage threshold & $5 \%$ \\
\hline
\end{tabular}


Notice that $10 \mathrm{~ms}$ is implemented to send two packets per frame, aiming to give more chances for DPCCH gating keeping still the packet delay under $80 \mathrm{~ms}$ but requiring to double the data rate in order to send two packets at once. In $2 \mathrm{~ms}$ TTI transmission the possible performance improvement does not justify the increase in data rate.

VI. System LeVEl SimUlation Results

Fig. 6 depicts the noise rise behavior from the system level simulation. Considering settled a $6 \mathrm{~dB}$ cell noise rise limit, gating gain around $42 \%$ and $50 \%$ is obtained for the $10 \mathrm{~ms}$ case and $41 \%$ for $2 \mathrm{~ms}$. The system level performance is under the semi-analytical results in Fig. 2 where $50 \%$ and $64 \%$ gains for $10 \mathrm{~ms}$ and $40 \%$ for $2 \mathrm{~ms}$ were reached. The semi-analytical study assumes that there is not limit for the UE tx power, whereas in the actual system simulations the UE power shortage limits performance, as explained in [8] for the $2 \mathrm{~ms}$ continuous scenario.



Figure 6 Mean noise rise for different throughput values

A better gating performance was observed regarding the outage capacity in figure 7: $50 \%$ and $77 \%$ gating gains can be observed for the throughput values of $60 \%$ and $80 \%$, respectively. $2 \mathrm{~ms}$ shows lower gating gain $(47 \%)$ getting the $10 \mathrm{~ms}$ gating result quite close to the $2 \mathrm{~ms}$ performance.

The main drawback concerning gating is the effect that it implementation might cause to the power control performance. As the DPCCH would not be continuously transmitted, the fast power control algorithm will not be able to follow the changes in the channel characteristics during the inactivity periods. That is why the use of a power control preamble, previous to the reactivation of the data channels is contemplated in [1]. In ou study, no preambles are considered to analyze the effect of gating on power control.

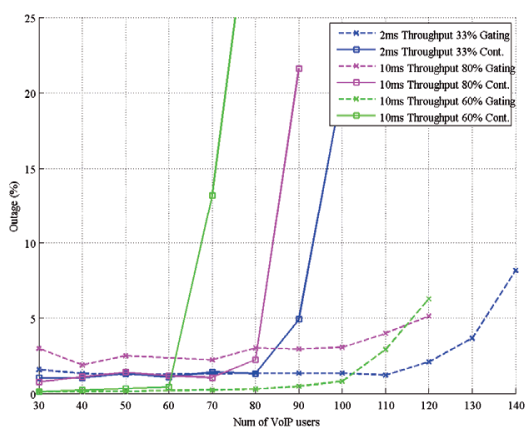

Figure 7 Cell outage for different throughput values

VII. CONCLUSIONS

In this paper we have considered the performance of $\mathrm{DPCCH}$ gating technique for HSUPA. Gating technique has recently been proposed within WCDMA to release unused resources by inactivating control channels during silent periods of the users. In order to study the concept benefit, Voice over IP was selected as the service under consideration. Both analytical and simulation studies were run to confirm the gain expectations. It was shown that VoIP would be clearly beneficed by the DPCCH gating inclusion in $3 \mathrm{GPP}$ specifications. Farther study concerning the use of power control preambles seems to be necessary to diminish the power control variability that the $\mathrm{DPCCH}$ gating technique introduces.

\section{REFERENCES}

[1] 3GPP TR 25.903 V1.0.0 (2006-05), "Continuous Connectivity For Packet Data Users"

[2] Y. Kim; J.-K Han; H. Kwon; D. Kim "Effect of imperfect power control the performance of HARQ 2050, Sept2004.

[3] ITU-T recommendation G.114, One way transmission time

[4] 3GPP TR 25.309 V6.4.0 (2002-06), "FDD Enhanced Uplink; Overall description".

[5] H. Holma, A. Toskala (Editors), "WCDMA for UMTS - Radio Access for Third Generation Mobile Communications", John Wiley and Sons, Third Edition, 2004.

[6] 3GPP TR 25.213 V6.4.0 (2005-09), " Spreading and modulation (FDD)".

7] 3GPP2 Technical Specification Group C, 3GPP2/TSG-C.1002: "1X EVDV Evaluation Methodology".

8] T. Chen; M. Kuusela; E. Malkamäki "Uplink Capacity of VoIP on HSUPA", VTC Spring 2006 


\title{
UPLINK DPCCH GATING OF INACTIVE UES IN CONTINUOUS PACKET CONNECTIVITY MODE FOR HSUPA
}

\author{
by \\ Chen, T., Malkamäki, E. and Ristaniemi, T. 2007 \\ Proceedings of IEEE Wireless Communications and Networking Conference 2007 \\ $\left(\mathrm{WCNC}^{\prime} 07\right)$
}

Reproduced with kind permission by IEEE 


\title{
Uplink DPCCH Gating of Inactive UEs in Continuous Packet Connectivity Mode for HSUPA
}

\author{
Tao Chen ${ }^{1}$, Esa Malkamäki ${ }^{2}$, Tapani Ristaniemi ${ }^{3}$ \\ ${ }^{1}$ Nokia Technology Platforms, ${ }^{2}$ Nokia Research Center, ${ }^{3}$ University of Jyväskylä \\ tao.t.chen@nokia.com
}

\begin{abstract}
In order to further improve the packet performance in the UMTS FDD system, uplink DPCCH gating as a scheme is proposed in 3GPP under the work item "Continuous connectivity for packet data users". In this paper, the uplink DPCCH gating concept is described and analyzed on both a qualitative and a quantitative level. And the system performance of different gating patterns used in the scheme was investigated by semianalytical prediction and system level simulations with different channel profiles. As will be shown, the selection of the gating pattern has a significant influence on the system performat patte is correlated with the uplink on which is correlated with the uplink data transmission activity and the uplink power contol. This contributes to a better understanding of the effects involved with uplink DPCCH gating where the biggest potential for performance optimizations can be found. In summary, the scheme of the uplink DPCCH gating is promising to improve the packet performance.
\end{abstract}

\section{Keywords- DPCCH gating, HSUPA, capacity}

\section{INTRODUCTION}

High-speed Uplink Packet Access (HSUPA), which is included in 3GPP Release 6 specification (see, e.g., [1],[2]), has been designed to increase the uplink packet data throughput by means of Hybrid Automatic Repeat request (HARQ), shorter frame size and fast scheduling controlled by the base station. High Speed Downlink Packet Access (HSDPA) is the downlink counterpart of HSUPA in 3GPP Rel'05 (see, e.g., [3]). Recently, there has been an increasing interest to further improve the packet service in the UMTS FDD system. In $3 \mathrm{GPP}$ the improvement of the packet performance is studied under the work item: "Continuous connectivity for packet dat users". Several concepts and methods have been proposed under this work item in order to: [4]

- Reduce the uplink noise rise from physical control channels of packet data users, e.g. for users which have temporarily no data transmission.

- Increase significantly in the UMTS FDD system the number of packet data users (i.e. HS-DSCH/E-DCH users without UL DPDCH) that can stay in CELL DCH state over a long time period, without degrading cell throughput, and that can restart transmission after a perio of inactivity with a much shorter delay $(<50 \mathrm{~ms})$ than would be necessary for reestablishment of a new connection.

- Allow improving the achievable UL capacity for VoIP users with its inherent periodic transmission through reducing the overhead of the control channels.
Subsequently, the Continuous Packet Connectivity (CPC) mode as a configuration of the CELL_DCH state has been proposed in 3GPP targeting to Rel'07. The CPC mode, where it is possible to transmit and receive discontinuously (i.e., only in a subset of UL and DL sub-frames), is sufficient especially for the light exchange of data (e.g. VoIP), or during inactive data transfer periods. In addition, as a premise of applying CPC mode, packet data users in CELL_DCH state using HSDPA and HSUPA as described in Rel'06 are addressed.

Uplink DPCCH gating, or discontinuous uplink DPCCH operation, as a candidate scheme applied in CPC mode for HSUPA has been proposed and investigated in 3GPP. In this paper, the gating pattern used in the uplink DPCCH gating as one open issue is studied. Moreover, the packet performance of the uplink DPCCH gating is evaluated on both a qualitative and a quantitative level. And VoIP related performance with uplink DPCCH gating is investigated in [5].

The rest of this paper is organized as follows. In Section II, the principle of uplink DPCCH gating is described, and realization in a HSUPA scenario is presented. Then, the semirealization in a HSUPA scenario is presented. Then, the semiprovides a brief overview of the used simulation model whereas the actual simulation results are presented in section IV. And finally, the conclusions are drawn in Section VI.

\section{UPLINK DPCCH GATING IN A HSUPA SCENARIO}

\section{A. Principle of uplink DPCCH gating}

Previously to 3GPP Rel'06, DPCCH channel was transmitted continuously regardless whether there is actual use data to be transmitted or not, thus highly loading the cell due to the DPCCH overhead of inactive packet users.

Thus, uplink DPCCH gating is proposed targeting to reduce DPCCH overhead of packet data users. The basic principle in short is that if there is neither E-DCH nor HS-DPCCH transmission, the UE automatically stops the continuous DPCCH transmission and applies a known DPCCH activity (DPCCH on/off) pattern, i.e., the gating pattern, in order to reduce DPCCH overhead of inactive users. With such solution the inactive packet data users kept in CELL DCH state would not consume any uplink air interface resource. And when an $\mathrm{E}$ the DPCCH is transmitted regardless of the gating pattern. An example of the uplink DPCCH gating is in Figure 1. 


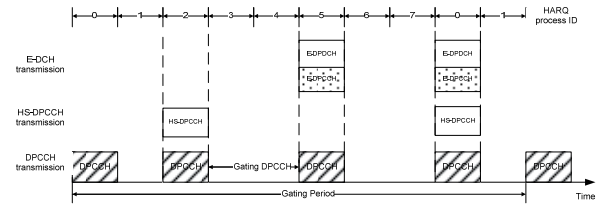

Figure 1. An example of the uplink DPCCH gating

The main advantages of uplink DPCCH gating can be summarized as below:

- Reducing the uplink interference from the physical contro channels of inactive packet data users

- Keeping more packet users in Cell_DCH state as long as possible to avoid the signaling delay from the radio link new establishment and state transitions, e.g. the transition between CELL_DCH and CELL_FACH.

- Power saving due to lowered battery consumption, which can result in the longer standby time of the UE.

- Easy operation mode transitions between Rel'06 active mode and CPC mode.

However, due to practical reasons there may be a limit on the length of the DPCCH gating period as during long inactivity the Node B could not any more know whether the uplink synchronization is lost or if there just is a very long uplink synchron
inactivity period.

Therefore the actual gating gain depends on several factors: the E-DCH activity, the HS-DPCCH activity and the gating pattern. The E-DCH activity is related with the data traffic and carries ACK/NACK messages for the HSDPA L1 HARQ transmissions and Channel Quality Indicator (CQI) messages for the HSDPA scheduling, is dependent on the downlink activity and SRNC configuration. Consequently, the gating activity and SRNC configuration. Consequently, the gating shall be set carefully to explore the most gating gain while keeping the uplink synchronization. For example, in the case of high activities on E-DCH/HS-DPCCH, the gating pattern can be set a small value.

\section{B. Realization of uplink DPCCH gating in a HSUPA} scenario

In the realization of uplink DPCCH gating, the key issue is how to perform the uplink power control in the case of the discontinuous uplink discontit carrier to interference (CIR) at a given CIR target. During the procedure of the continuous DPCCH transmission, the adjustment to the DPCCH power is determined by UE based on the previous value that used in the previous slot, whereas in the gating mode, when there is an interruption in transmission, the powe of the DPCCH is derived according to the previous value that used in the last slotbefore the transmission gap. However, after a gating period without tracking the received SIR, the channel increase the interference between the UEs. Consequently, optional power control preambles are defined to be sent previous to the data channel reactivation, which is a period of uplink DPCCH transmission prior to the start of the uplink DPDCH transmission. Besides, the uplink power control during the uplink DPCCH gating can use Power Contro Algorithm (PCA) 1 and a 1dB step size as described in [6]

In addition, it is interesting to point out here that the $\mathrm{L}$ synchronous HARQ protocol specified for HSUPA helps to decrease the impact of inaccuracies in the power control algorithm as illustrated in [7], and thus it will help with the gating technique.

Further, a general scenario of uplink DPCCH gating in HSUPA as shown in Figure 2 is proposed for the study. Both active packet users with the full buffer traffic and inactive users without any data transmission on E-DCH/HS-DPCCH exist simultaneously. The gating pattern is applied on inactive users. Further, the gating pattern modeled here can be also understood as L1 synchronous HARQ transmissions, Preambles, periodica CQI reporting on HS-DPCCH or the transmissions of the periodical service, e.g., VoIP,

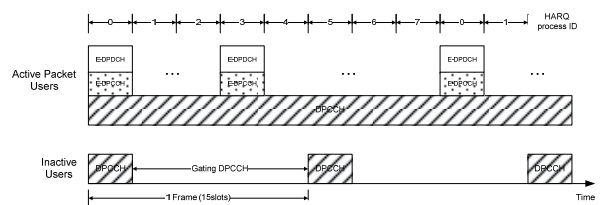

Figure 2. A scenario of uplink DPCCH gating in HSUPA

III. SEMI-ANALYTICAL StUdy

In the semi-analytical study, we derive the cell Noise Rise (NR) for the capacity evaluation in HSUPA from the following typical uplink load formula (see, e.g., [8])

$N R_{d B}=-10 \log 10\left(1-\frac{E b N o}{W / R} N \cdot v \cdot(1+i)\right), \quad$ (1)

where $N$ is the number of users per cell, $v$ is the average activity factor, EbNo is the signal energy per bit divided by noise spectral density, $W$ is the chip rate, $R$ is the data rate, $i$ is noise spectral density, $W$ is the chip rate, $R$ is the data rate, $i$ is
the other cell to own cell interference ratio, and $N R_{d B}$ is the Noise Rise in dB.

Considering UEs with different activity factors in HSUPA i.e., different activity factors on physical channels, the NR described above can be further expressed as

$N R_{d B}=-10 \log 10\left(1-\sum_{j=1}^{N}\left(E c N o_{c}(j) \cdot v_{c}(j)+E c N o_{e}(j) \cdot v_{e}(j)\right) \cdot(1+i)\right)$,

where $E c N o_{c}(j)$ is the signal energy per chip divided by noise spectral density on DPCCH for the $j$ th UE, $v_{c}(j)$ is the activity factor on DPCCH for the $j$ th UE, $E b N o_{e}(j)$ is the EcNo on EDCH for the $j$ th UE, $v_{e}(j)$ is the activity factor on E-DCH for the $j$ th UE. Consequently, $v_{e}(j)$ would be zero for the inactive UE, which is considered to have the transmission only on DPCCH, i.e., no transmission on E-DCH. However for active 
UEs with the data transmission on E-DCH, $v_{e}(j)$ is a non-zero value. For example, $v_{e}(j)$ would be one for the UE with the full buffer traffic, which may transmit the data every TTI on E$\mathrm{DCH}$ associated with DPCCH. In addition, $v_{c}(j)$ shall be one for any UE definitely in Rel'6, whereas can vary with differen DPCCH gating patterns in the CPC mode for the inactive UE. Further, due to the fact that E-DCH resources are always allocated by the scheduling algorithm after the DPCCH resource allocation, the impact of inactive UEs in the CPC resource allocation, the impact of inactive UEs in the CPC
mode with only DPCCH transmissions on the capacity can be evaluated based on the NR of inactive UEs, denoted as

$N R_{d B_{\text {Inactive }}}=-10 \log 10\left(1-N_{\text {inactive }} E c N o_{c} v_{c} \cdot(1+i)\right)$

where $N_{\text {inactive }}$ stands for the inactive UE number. Note that al inactive UEs have the same $E c N o_{c}$ and $v_{c}$ in order to simplify the study. Therefore, the radio resource allocated to DPCCH transmissions for inactive UEs can be expressed as a function of $N_{\text {inactive, }} E c N o_{c}$ and $v_{c}$. Thus, the NR contributed by inactive UEs can be calculated as shown in Figure 3 assuming $i$ is 0.65 Es can be calculated as $\mathrm{ESN}_{c}$ is at and the requirement of $E_{c} N o_{c}$ is about $-18 \mathrm{~dB}$ for the inactive UE.

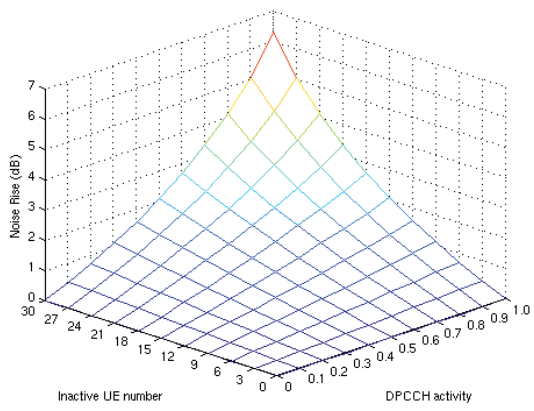

Figure 3. Noise Rise as a function of the inactive UE number and the DPCCH activity factor

Clearly, up to 30 inactive users with the continuous DPCCH transmission can eat up all reserved radio resource, i.e., $6 \mathrm{~dB}$ noise rise target. On the other hand, less DPCCH activity or less inactive UE number can reduce the uplink interference dramatically. In other words, the system can offer more inactive UEs by reducing the DPCCH activity of these more in UEs. For the DPCCH activity factor of 0.2 , which implies that DPCCH gating in the CPC mode is a promising approach to reduce the uplink interference, and thus lead to higher capacity and longe staying of the inactive UE in the Cell_DCH state. Ideally, fully gating on DPCCH for inactive UEs can reach the highest capacity gain. Unfortunately, it's impossible to avoid DPCCH transmission totally for the uplink inactive users due to the requirements of the uplink synchronization and TPC commands for the inner loop power control of the downlink FDPCH in Rel'6 or the HS-DSCH associated DCHs in Rel'5 in the case of the active downlink.

\section{Modelling AND Assumptions}

\section{A. E-TFC selection}

In WCDMA uplink transmission, UEs have the control of selecting which data rate combination to use. The algorithm for the decision is called TFC elimination/selection for Rel' 99 $\mathrm{DCH}$, and E-TFC elimination/selection for the HSUPA EDCH. UEs in CELL_DCH state with an E-DCH transport channel configured shall apply the E-TFC selection procedure when invoked by the HARQ entity. Briefly, E-TFC selection including the scheduling process can be illustrated as below. Firstly, the UE will send Scheduling Requests (SR) in the form of Scheduling Information (SI) and happy bit to Node-B to indicates the UE requesting resource from Node B. Secondly, Node-B will decide the maximum allowed data rate for each UE with the priority function, i.e. scheduling algorithms, based on SIs from UEs, UEs' capabilities and the current interference level. Then Node-B will send Scheduling Grant (SG) signaling to inform the UE the maximum power for the upcoming suitable for the current status and not requiring higher power

\section{B. Channel modeling}

Three types of uplink dedicated physical channels, the uplink DPCCH, the E-DPDCH and the E-DPCCH, are modeled. The DPCCH will carry control information generated at Layer 1, which consists of known pilot bits to support channel estimation for coherent detection, and transmit powercontrol (TPC) commands. Optional feedback information (FBI), and an optional transport-format combination indicator (TFCI) are not assumed here. One E-DPCCH on each radio link is modeled as the overhead. In order to ensure E-DPCCH link is modeled as the overhead. In order to ensure E-DPCCH transmission error free, the power ratio between E-DPCCH and
DPCCH, denoted as $\left(\beta_{c c} / \beta_{c}\right)^{2}[6]$, is selected at $3 \mathrm{~dB}$ based on the link level simulation study. One E-DPDCH on each radio link is modeled to carry the E-DCH transport channel. The data transmitted by E-DPDCH will experience the synchronous L1 transmitted by E-DPDCH will experience the synchronous L1 DPDCH and DPCCH denoted as $\left(\beta_{e d} / \beta_{c}\right)^{2}$ at $8 \mathrm{~dB}$ is applied as an optimized value obtained from the link level simulation study. Therefore, the total transmission power $P_{\text {Total }}$ for the active UEs can be expressed as

$$
P_{\text {Total }}=P_{D P C C H} \cdot\left(1+\left(\frac{\beta_{e c}}{\beta_{c}}\right)^{2}+\left(\frac{\beta_{e d}}{\beta_{c}}\right)^{2}\right),
$$

where $P_{D P C C H}$ is the DPCCH power. Note that the E-DPDCH and E-DPCCH are always frame aligned with each other. However, the total transmission power for inactive UEs will not include E-DCH transmission power.

\section{Environment}

A quasi-static system level simulator, where all necessary RRM algorithms as well as their interactions are modeled, is used to investigate the impact of the DPCCH gating on the capacity. This tool includes a detailed simulation of the user within multiple cells based on the descriptions in [10]. The fast fading is explicitly modeled for each user according to the ITU 
Pedestrian-A profile and ITU Vehicular-A profile. ITU Pedestrian-A channel model is used in these simulations to study the effect of low multipath diversity case. ITU Pedestrian A channel is close to 1-path channel and does not give much multipath diversity while ITU Vehicular A channel gives quite significant multipath diversity. Otherwise, the link level performance at the system level for the full buffer UE is modeled with Eb/N0 to BLER mapping, called the "Actual Value Interface" (AVI), described in [11].

Main parameters used in the system simulation are summarized in Table I

TABle I. Parameters Settings in the SimUlation

\begin{tabular}{|l|l|}
\hline \multicolumn{1}{|c|}{ Parameter } & \multicolumn{1}{c|}{ Value } \\
\hline Frame Size & $2 \mathrm{~ms} \mathrm{TTI}$ (1 Subframe) \\
\hline Inter Site Distance & $2.8 \mathrm{~km}$ \\
\hline Cell Configuration & ITU Veh-A / Ped-A, Macrocell \\
\hline UE Speed & Veh-A 30kmph / Ped-A 3kmph \\
\hline Noise Rise Target & $6 \mathrm{~dB}$ \\
\hline \multicolumn{2}{|c|}{ Active Packet Users Setting } \\
\hline Traffic Model & Full Buffer \\
\hline UE Number & 6 UEs \\
\hline E-TFC Set & $\{64,128,256,384,512,1024\} \mathrm{kbps}$ \\
\hline Number of HARQ Channels & 8 \\
\hline Max Number of L1 Transmissions & 4 \\
\hline $\left.\boldsymbol{\beta}_{\boldsymbol{c}} / \boldsymbol{\beta}_{c}\right)^{2}$ & $3 \mathrm{~dB}$ \\
\hline Residual FER Target & $1 \%$ \\
\hline \multicolumn{2}{|c|}{ Inactive Users Setting } \\
\hline Traffic Model & No data traffic \\
\hline $\begin{array}{l}\text { DPCCH Gating Pattern } \\
\text { (Gated slots / Total 15slots) }\end{array}$ & NotGated, 9/15, 12/15 \\
\hline DPCCH CIR Target & $-18.1 \mathrm{~dB}$ \\
\hline Inactive UE Number & $\{0,10,15,20, \ldots, 50\}$ UEs \\
\hline
\end{tabular}

Note that active packet UEs with the full buffer traffic will not have any discontinuous DPCCH transmission. And thereby, the DPCCH gating is only applied on inactive UEs with different gating patterns.

\section{System Level Simulation Results}

The impact of the gating on DPCCH for inactive UEs in the CPC mode, based on the considerations described above, has been evaluated.

Figure 4 and Figure 5 show the significant improvement in the cell throughput with gating DPCCH on inactive UEs for PA3 and VA30 channel profiles separately. Note that ideal reference curves for gating patterns have been calculated simply by scaling the continuous DPCCH curves with the gating ratio. In the case of 20 inactive UEs, the cell throughput from 6 active packet UEs is only 500kbps without DPCCH gating on inactive UEs, which is just around the half of the cell throughput with the gating pattern of $9 / 15$ on inactive UEs. Moreover, it can be found that more gating on DPCCH will lead to more gain in the cell throughput when comparing cases of the gating pain of the gating pattern $12 / 15$ and $9 / 15$. In addition, it is noted th with the semi-analytical study, i.e., at most about 30 inactive
UEs without the DPCCH gating can be offered in the system assuming $6 \mathrm{~dB}$ NR target.

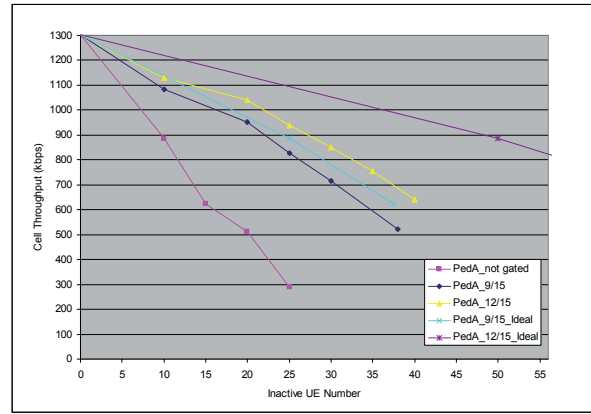

Figure 4. Cell throughput as a function of inactive UEs, different gating patterns vs. ideal gating, PA3

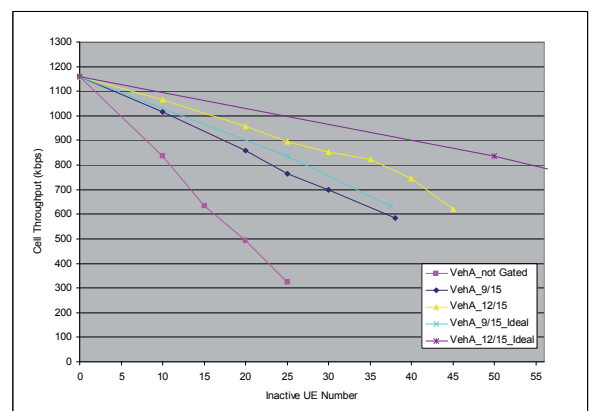
Figure 5. Cell throughput as a function of inactive UEs, different gating

Even if the clear gating gain on the cell throughput can be obtained, unfortunately, it can be limited by the loss from the discontinuous inner loop power control on DPCCH, which may degrade the capability of UE to follow the fast changing channel conditions. Comparing gating cases with and without the ideal power control, this kind of power control loss is shown implicitly. For example, in the case of 40 inactive UEs with the gating pattern $12 / 15$ in PA3, the cell throughput is approximately $800 \mathrm{kbps}$, which is only two third of what it is performed with the ideal power control.

Further, the overshoot, i.e., the probability of noise rise higher than $7 \mathrm{~dB}$ during the simulation, is collected in Figure 6 , which shows lower overshoot for gating cases at the same inactive UE number. The overshoot curves for VA30 are flat whereas PA3 overshoot curves show the fast changing, which implies that ideal power control is more important for $\mathrm{PA} 3$ th the non-ideal power control will less impact VA30 than PA3. 
Thus, more gating on DPCCH for VA30 can be considered in order to reach higher cell throughput.

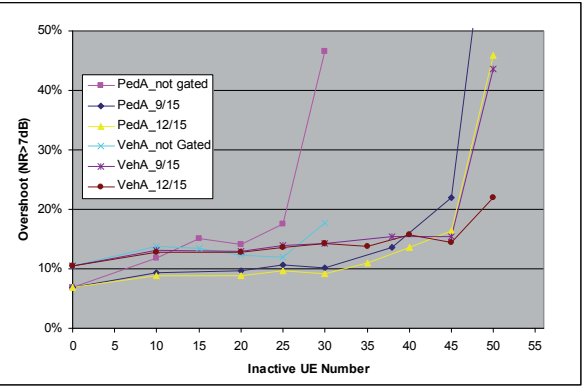

Figure 6. NR Overshoot as a function of inactive UEs, different gating patterns and channel models

On the other hand, more details about the benefit to active UEs from inactive UEs' gating are presented in Figure 7 . Clearly, due to the less interference in the uplink, more radio resource can be reserved and allocated to active packet UE based on the operation of E-TFC selection. Consequently, the high bitrate E-TFC such as $1024 \mathrm{kbps}$ is chosen with the highe probability of up to $35 \%$ for the gating pattern $12 / 15$ than others. Otherwise, the low bitrate E-TFC will be more selected for the non-gating case, e.g, with the probability of more than $60 \%$. Thus, it matches well with the performance of the cell throughput.

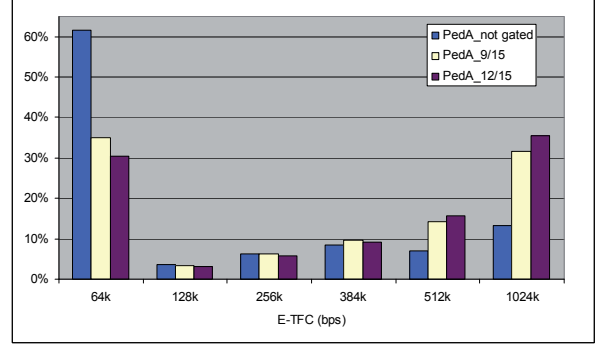

Figure 7. Distribution of E-TFC bitrates of active UEs as a function of the gating pattern with 6 active UEs and 20 inactive UEs per cell, PA

In order to analyze the performance from the UE point of view, the average UE throughput of the active UE is presented in Figure 8. Approximately $50 \%$ gating gain on the UE throughput can be achieved with the gating pattern $9 / 15$ compared to the non-gating cases, and even more gain for the compared to the non-gating cases, and even more gain for the
gating pattern $12 / 15$. Subsequently, the improvement on the gating pattern $12 / 15$. Subsequently, the improvem
UE throughput from the gating is significant as well.

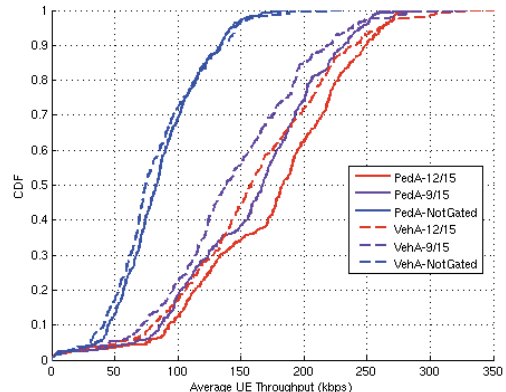

Figure 8. Average UE throughput distribution with 6 active UEs and 20 inactive UEs per cell, different gating patterns and channel models

\section{Conclusions}

In this paper, we have considered the effects involved with uplink DPCCH gating in CPC mode on the HSUPA system performance. And we analyzed the system performance, which is investigated by semi-analytical prediction and system leve simulations. The actual gating gain on the performance depends on the activity in the uplink transmission and the quality of uplink power control. The loss from the uplink ous uplink DPCCH transmission may limit the gating gain from the DPCCH gating. And in different channel profiles, the effects of the tradeoff between the DPCCH gating gain and the loss from the discontinuous power control on the system performance can be different, which depends on the requirement on the power control. Therefore, the optimization of the gating pattern should be considered to explore the most gating gain. Generlly speaking, the scheme of the uplink DPCCH gain. shows a significant improvement on the uplink system performance.

\section{REFERENCES}

[1] 3GPP TS 25.309, "FDD Enhanced Uplink; Overall description; Stage 2"

[2] 3GPP TR 25.896, "Feasibility Study for Enhanced Uplink (FDD)",

[3] 3GPP TS 25.308, "HSDPA; Overall description; Stage2".

4] 3GPP TR 25.903, "Connectivity for Packet Data Users".

[5] Oscar Fresan, Tao Chen, Esa Malkamäki, Tapani Ristaniemi, "DPCCH Oscar Fresan, Tao Chen, Esa Malkamaki, Tapani Ristaniemi, "DPCC

[6] 3GPP TS 25.214, "Physical layer procedures (FDD)".

[7] Y. Kim, J.-K Han, H. Kwon, D. Kim "Effect of imperfect power control on the performance of HARQ in CDMA2000 reverse link", 15th

[8] H. Holma, A. Toskala (Editors), "WCDMA for UMTS", John Wiley and Sons, Third Edition, 2004

[9] F. Frederiksen, T.E. Kolding, "Performance and Modelling of WCDMA/HSDPA transmission/H-ARQ Schemes", IEEE VTC, Fall, pp.472-481, 2002.

[10] 3GPP2 TSG-C.1002: "1X EV-DV Evaluation Methodology"

[11] Hämäläinen S., P. Slanina, M. Hartman, A. Lappeteläinen, H. Holma, O. Salonaho, "A Novel Interface between Link and System Leve Simulations", Proceedings of ACTS summit 1997, Aalborg, Denmark 


\title{
VOIP END-TO-END PERFORMANCE IN HSPA WITH PACKET AGE AIDED HSDPA SCHEDULING
}

\author{
by \\ Chen, T., Charbit, G., Ranta-aho, K., Fresan, O. and Ristaniemi, T. 2008 \\ Proceedings of $19^{\text {th }}$ IEEE International Symposium of Personal, Indoor and Mobile \\ Radio Communications Journal (PIMRC'08)
}

Reproduced with kind permission by IEEE 


\title{
VoIP End-to-End Performance in HSPA with Packet Age Aided HSDPA Scheduling
}

\author{
${ }^{1}$ Tao Chen, ${ }^{2}$ Gilles Charbit, ${ }^{3}$ Karri Ranta-aho, ${ }^{4}$ Oscar Fresan, ${ }^{5}$ Tapani Ristaniemi \\ ${ }^{1,2}$ Nokia Devices, ${ }^{3}$ Nokia Siemens Networks, ${ }^{4}$ University of Tampere, ${ }^{5}$ University of Jyväskylä \\ tao.t.chen@nokia.com
}

\begin{abstract}
In this paper, we present an enhanced VoIP scheduling for the high speed downlink packet access (HSDPA) in UMTS, which takes the age of the VoIP packet into account. The downlink capacity can be significantly improved by this way, especially for shorter uplink transmission delay. In order to quantify the achievable performance improvement, we present quantify the achievable performance improvement, we present
results obtained from extensive system-level uplink and downlink
simulations. Inter alia, it is shown that using the proposed scheme can lead to an increase in the downlink cell capacity of up to $16 \%$. By applying the proposed method, the downlink perfor uplink performance remains the same, which ensures the good end-to-
end performance.
\end{abstract}

Index Terms-Scheduling, VoIP, HSUPA, HSDPA

\section{INTRODUCTION}

$\mathrm{H}$

igh-speed Uplink Packet Access (HSUPA), which is included in 3GPP Release 6 specification (see, e.g., [1],[2]), has been designed to increase the uplink packet data throughput by means of Hybrid Automatic Repeat request (HARQ), shorter frame size and fast scheduling controlled by the base station. High Speed Downlink Packet Access (HSDPA) is the downlink counterpart of HSUPA in 3GPP Release 5 (see, e.g., [3]). Furthermore, both HSUPA and HSDPA have been improved in Release 7, which is so called High-speed Packet Access (HSPA). Recently, there has been an increasing interest to use HSDPA/HSUPA for real-time (RT) packet switched (PS) services such as Voice over IP (VoIP). The reasoning behind the increased interest towards VoIP is to use VoIP in All-IP network instead of using circuit switched (CS) speech, which will imply cost savings for operators as CS related part of the core network would not be needed anymore. The challenge for the VoIP on HSPA will be the delay requirement for the voice traffic.

Some enhanced HSDPA VoIP schedulers considering the packet delay requirement have been developed to offer VoIP services more efficiently. However, this kind of static delay budget used for scheduling is mainly based on a rough estimation, which is typically not accurate enough due to the retransmissions and varying channel conditions. Furthermore, an overestimated delay budget may lead to poor end-user

978-1-4244-2644-7/08/\$25.00 (2008 IEEE experience, whereas an underestimated delay budget may sacrifice the radio efficiency. Therefore, a packet age aided scheduling scheme is proposed in this paper by exploiting the packet delay information in order to improve VoIP performance over HSPA.

The rest of this paper is organized as follows. In Section II, the principle of VoIP over HSPA is described, and an enhanced scheduling scheme in HSDPA aided by the packet age is proposed. Then, the system modeling is described in Section III whereas both uplink and downlink simulation results are presented and analyzed in section IV. And finally, the conclusions are drawn in Section VI.

\section{PRINCIPLE OF VOIP OVER HSPA}

\section{A. VoIP Packet Delay requirement}

VoIP as a real-time service requires the transmission and reception within the reasonable time. According to [4], the maximum acceptable mouth-to-ear delay for voice is on the order of $250 \mathrm{~ms}$. If the delay for core network, RNC processing and Iub transport is approximately $100 \mathrm{~ms}$, the one-way tolerable delay for MAC buffering/scheduling , transmission and reception would be roughly $150 \mathrm{~ms}$ assuming VoIP call is between two mobiles. Furthermore, this maximum allowed delay is typically used as the delay budget for the evaluation of VoIP service over HSPA.

\section{B. VoIP over HSPA}

HSPA was originally designed to carry high bit rate delay tolerant data. A number of features have been introduced to 3GPP Release 6 and 7 to improve the efficiency of low bit rate delay critical applications like VoIP. In [5], the VoIP enabling features such as packet bundling, uplink gating for HSUPA, non-scheduled HSUPA transmission and advanced HSDPA receivers are summarized. Uplink gating, which will disable DPCCH transmission when there is no data to be sent on EDCH and no L1 feedback signaling on High-speed Dedicated Physical Control Channel (HS-DPCCH), can minimize the L1 control overhead, reduce uplink interference and save UE power consumption. Packet bundling by sending multiple VoIP packets together can minimize the control overhead improving the performance in both uplink and downlink.

Besides, some VoIP delay sensitive scheduling algorithms in HSDPA (see [6], [7]) were proposed and evaluated 
previously, in which the power allocation, codes allocation and user multiplexing are scheduled considering the VoIP packet delay requirement as well as the channel conditions. The channel conditions are obtained from the Channel Quality Indicator (CQI) report transmitted on uplink HS-DPCCH channel. The packet delay requirement used for the scheduling is just a static delay budget, e.g., $80 \mathrm{~ms}$, which is based on a rough estimation by dividing $150 \mathrm{~ms}$ end-to-end air interface delay budget into uplink and downlink components. It has been proved that taking the packet delay requirement into account in the downlink scheduling can improve the VoIP performance while retaining the service quality (see [7]).

\section{VoIP PACKET Age AIDEd HSDPA SCHEDULING}

Even though early scheduling schemes provides some improvement in the downlink capacity by considering the packet delay requirement, the estimated static delay budget applying for all VoIP packets may not be reasonable, because it is supposing that all VoIP packets would suffer similar delay before arriving at NodeB scheduling queue. Hence, there is no difference on the priority in the terms of the packet delay for VoIP packets. Furthermore, this kind of static delay budget leads to the independent downlink scheduling without considering any end-to-end user experience. For example, the packet suffering shorter uplink delay can be transmitted in the downlink earlier than the packet suffering longer uplink delay due to the better channel condition and the same delay budget. Further, it may result in the outage of the latter because the overall delay may exceed $150 \mathrm{~ms}$ even though the downlink transmission is done successfully within $80 \mathrm{~ms}$ static delay budget. Besides, each packet arriving at NodeB scheduling queue may suffer different delay in practice. The suffered delay would vary with the uplink sources. For example, the uplink delay from the mobile would be larger than from the fixed-line phone because of the retransmission in the complicated channel conditions. Even for mobiles there can still be some difference on the uplink transmission delay due to the varying channel conditions. Therefore, it does make sense to exploit the specific delay budget for each packet in order to fully utilize the radio resource efficiently for higher VoIP capacity while maintaining end-user good experience.

Here, an enhanced HSDPA scheduling aided by the packet age is proposed. The downlink delay budget used for scheduling would be derived from the one way end-to-end delay budget and the "age" of the VoIP packet as expressed in equation (1)

$$
P D B_{u i}^{p j}=E 2 E_{u i}^{p j}-P A g e_{u i}^{p j}=250 m s-P A g e_{u i}^{p j}
$$

where $P D B_{u i}^{p j}$ stands for the delay budget used in the downlink scheduling for packet $j$ of user $i, E 2 E_{u j}^{p j}$ the end-toend delay budget for packet $j$ of user $i$ and $P A g e_{u i}^{p j}$ the age of packet $j$ of user $i$. Here, the end-to-end delay budget is the maximum acceptable mouth-to-ear delay. The packet age is defined as the accumulative delay from the generation of the
VoIP packet to the instant of packet arriving at the end-user serving NodeB in the downlink.

Due to the trend of migrating to the ALL-IP network, the packet age information can be derived from the time-stamp in the IP header. The birth of the packet can be marked by UE in the uplink through the IP layer or by the calling node in the IP network. In IP header (see [8]), there is an option for the internet timestamp in milliseconds as shown in Table I. TABLE I.

\begin{tabular}{|l|l|l|l|l|}
\hline 1000100 & Length & Pointer & Oflw & flg \\
\hline \multicolumn{5}{|c|}{ Internet address } \\
\hline \multicolumn{5}{|c|}{ Timestamp } \\
\hline
\end{tabular}

Thus, for example, the UE packet timestamp information in 3G-GGSN (see Fig. 1) could firstly be obtained by calculating the accumulative delay according to the time difference between current time and the packet timestamp. Then, the timestamp information can subsequently be appended for each hop. Finally, the NodeB may derive the age of the each packet according to IP header in the case of direct IP connection to NodeB, assuming that most RNC functions have been moved to Node-B and IP transmission is supported.

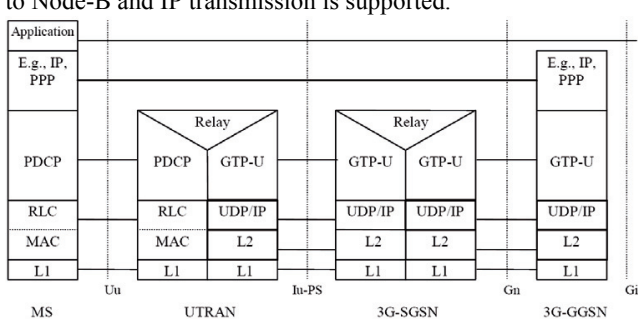

UTRAN
Fig. 1. 3GPP protocol stack (see [9]) $3 G \operatorname{GGSY}$

If RNC and NodeB functions are split and Iub IP transmission is not supported, we may attach the age information into the VoIP packet via the extended fields of frame protocol.

Each VoIP UE can have its own specific delay budget for HSDPA scheduling. The serving NodeB can prioritize packets to the UEs based on the specific delay budget of each packet. Otherwise, the scheduler can even discard the packet directly when the packet age is exceeding the $150 \mathrm{~ms}$ end-to-end delay budget. So it is a better use of resources to avoid transmitting a VoIP packet that is known to have higher end-to-end delay than what is required for acceptable end-user experience.

The scheduling for VoIP users aided by the packet age is such that, it selects at most M users, which is corresponding to the maximum allowed multiplexing user number, from a scheduling candidate set (SCS) by using Proportional Fair (PF) packet scheduling algorithm which exploits the instantaneous channel state information for each user, and therefore provides a multi-user diversity gain. The selected VoIP users are to be transmitted in the following $2 \mathrm{~ms}$ Transmission Time Interval (TTI).

The SCS includes VoIP users that fulfill the following 
criteria:

(i) Users whose packet delay is equal to or larger than threshold corresponding to the packet specific delay budget.

(ii) Users with pending retransmissions in their hybrid automatic repeat request (HARQ) manager.

If $\mathrm{M}$ users are not fulfilled by SCS, other users can be selected according to the priority of PF scheduling.

The novelty of the proposed scheme is two-fold

1. The packet specific delay budget for each VoIP packet will be used instead of the static delay budget by considering the "age" of the packet.

2. The age information can be obtained from the optional fields of the IP header assuming IP connection to NodeB in the transmission network. Alternatively without IP connection to the NodeB, the RNC could inform the NodeB, for example, by appending similar information to each VoIP packet via some extended fields of the frame protocol.

With this proposed delay sensitive scheduling the other end HSDPA scheduler can benefit from the knowledge that some packets arrived faster to the buffer than some other packets, and it can thus have longer scheduler delay for those arriving faster. Hence, it is more viable than the conventional method with the static radio delay budget as described in prior art in which the scheduling may not consider the specific end-user experience. It can also dejitter the buffer delay by scheduling users more efficiently according to the packet specific end-toend delay budget. Consequently, it can enhance downlink radio transmission efficiency. Otherwise, the method could be widely used in other IP-based system such as Long Term Evolution (LTE) system and Wimax for the downlink scheduling of real-time traffic.

\section{MODELING OF VOIP OVER HSPA}

\section{A. VoIP traffic}

The duration of each VoIP call is sampled from a negative exponential distribution function with an average call length of 60 seconds. Discontinuous transmission (DTX) is simulated by $50 \%$ probability of transmission and silent periods, i.e., on and off periods. The duration of both on and off periods is negative exponentially distributed with an average of 3 seconds. During on periods, a voice encoder generates a payload per VoIP packet of 31 bytes every $20 \mathrm{~ms}$, corresponding to a source rate of approximately $12.2 \mathrm{kbps}$. Another 9 bytes from the headers of RTP/UDP/IP/PDCP/RLC with header compression will be included in each VoIP packet.

\section{B. VoIP QoS}

The outage is introduced to evaluate the performance of VoIP on HSPA. Here, an outage is counted if $5 \%$ of the VoIP packets for one user are not received within the delay budget when monitored over a 10 seconds on period.

Each newly arrived VoIP packet in the UE is associated with a discard timer. Whenever a buffered packet has been transmitted, it is moved to the Hybrid-ARQ manager and its discard timer is deactivated. Hence, whenever a packet has been transmitted, it can be dropped only if it has not been successfully received after the maximum number of allowed transmissions.

Furthermore, the capacity is defined as the number of VoIP users per sector without exceeding $5 \%$ cell outage level. The typical criterion for the uplink, noise rise of the sector, is also applied for the benchmarking of uplink results.

\section{HSPA system level simulators}

The quasi-static system level simulators respectively for HSUPA and HSDPA, where all necessary RRM algorithms as well as their interactions are modeled, are used to investigate the performance of VoIP on HSPA with the proposed method. These tools include a detailed simulation of the users within multiple cells. The fast fading is explicitly modeled for each user according to the ITU Vehicular-A profile. Regarding to the methodology this kind of quasi-static simulator is based on descriptions in [10]. A wrap-around multi-cell layout modeling several layers of interference is utilized in this study.

However, different with the conventional VoIP simulations with independent uplink and downlink simulators, both simulators would be coupled by the delay information, i.e., the uplink packet delay from the uplink simulation result would be used as the input of the downlink simulation in order to calculate the packet specific delay budget for the downlink scheduling.

On the other hand, CQI reporting on $10 \mathrm{~ms}$ period is modeled, which is supposed to provide good balance between the uplink interference from CQI reporting and efficient downlink scheduling based on CQI information. Therefore, uplink interference from HS-DPCCH carrying $10 \mathrm{~ms}$ CQI report and CQI information available on $10 \mathrm{~ms}$ period for downlink scheduling are modeled in HSUPA and HSDPA simulators separately to study the end-to-end performance in a practical way.

\section{SimUlation SCEnario and Results Analysis}

Main parameters used in the system simulation are summarized in Table II.

TABLE II.

SIMULATION PARAMETERS SETTINGS

\begin{tabular}{|l|l|}
\hline \multicolumn{1}{|c|}{ Parameter } & \multicolumn{1}{c|}{ Value } \\
\hline \multicolumn{2}{|c|}{ System configuration } \\
\hline Inter site distance & $2.8 \mathrm{~km}$ \\
\hline Cell configuration & ITU Veh-A, Macrocell \\
\hline Voice call mean length & 60 seconds \\
\hline UE speed & $3 \mathrm{kmph}$ \\
\hline Outage observation window length & $10 \mathrm{~seconds}$ \\
\hline Cell outage threshold & $5 \%$ \\
\hline End-to-End air interface delay budget & $150 \mathrm{~ms}$ \\
\hline Residual FER HSUPA specific configuration \\
\hline \multicolumn{2}{|c|}{$1 \%$} \\
\hline Frame Size & $2 \mathrm{~ms} / 10 \mathrm{~ms}$ \\
\hline Channels & E-DCH/DPCCH \\
\hline Number of HARQ channels & $8(2 \mathrm{~ms} \mathrm{TTI} / 4$ (10ms TTI) \\
\hline
\end{tabular}




\begin{tabular}{|l|l|}
\hline Max number of L1 transmissions & $4(2 \mathrm{~ms} \mathrm{TTI}) / 2(10 \mathrm{~ms} \mathrm{TTI})$ \\
\hline Uplink delay budget & $80 \mathrm{~ms}$ \\
\hline Scheduling algorithm & Non-scheduled \\
\hline \multicolumn{2}{|c|}{ HSDPA specific configuration } \\
\hline Frame Size & $2 \mathrm{~ms} / 10 \mathrm{~ms}$ \\
\hline Channels & HS-DSCH/CPICH/HS-DPCCH \\
\hline Number of HARQ channels & $8(2 \mathrm{~ms} \mathrm{TTI}) / 4(10 \mathrm{~ms} \mathrm{TTI})$ \\
\hline Max number of L1 transmissions & $4(2 \mathrm{~ms} \mathrm{TTI}) / 2(10 \mathrm{~ms} \mathrm{TTI})$ \\
\hline Max CDM Users & 4 \\
\hline Number of code & 10 \\
\hline HSDPA power / Max Power & $12.5 \mathrm{w} / 20 \mathrm{w}$ \\
\hline Downlink delay budget & $(150 \mathrm{~ms}-$ UplinkPacketDelay $)$ \\
\hline
\end{tabular}

\section{A. Performance of VoIP over HSUPA}

In a first step, we investigated the VoIP performance that can be achieved by applying HSUPA features for different TTI lengths. In Table III, the uplink VoIP capacity results with two criteria for both $2 \mathrm{~ms}$ and $10 \mathrm{~ms}$ TTI lengths are summarized. It is observed that uplink gating can give $40-50 \%$ further gain compared to the case of continuous DPCCH transmission, whatever the TTI length and QoS criteria are.

As can be seen, for uplink gating with average 1.25 HARQ transmissions in $10 \mathrm{~ms}$ TTI length, the performance gain in terms of the cell capacity is higher compared to the case of average 1.67 HARQ transmissions. That is because fewer transmissions may spare more time for DPCCH gating, which can further lead to less uplink interference. Consequently, it provides more potential for significant performance improvement with DPCCH gating.

On the other hand, fewer transmissions would require higher power leading to the power shortage of the UE in the cell edge. This can result in capacity loss as shown in the case of $10 \mathrm{~ms}$ TTI with continuous DPCCH transmission, where the capacity is 65 UEs per sector for 1.25 transmissions whereas 80UEs for 1.67 transmissions in terms of the cell outage criteria.

Thus, FER target setting after 1st transmission used in outer loop power control can be adjusted to optimize the average HARQ transmission in the case of DPCCH gating for exploring the potential capacity gain.

In Fig. 2, some uplink packet delay information is collected for both cases of DPCCH gating and no gating (i.e., continuous DPCCH transmission). It is noted that uplink packet transmission can always be done within the uplink delay budget (i.e., $80 \mathrm{~ms}$ ). The most reason is that synchronous HARQ transmission in HSUPA would always force the packet transmission in the period, which can ensure the packet delay not to exceed some threshold in accordance with the maximum allowed HARQ transmission number. It is proved in the case of $2 \mathrm{~ms}$ TTI that there are 4 slopes corresponding to 4 HARQ transmissions. Therefore, it also implies that the packet delay is not the bottleneck for the uplink capacity.

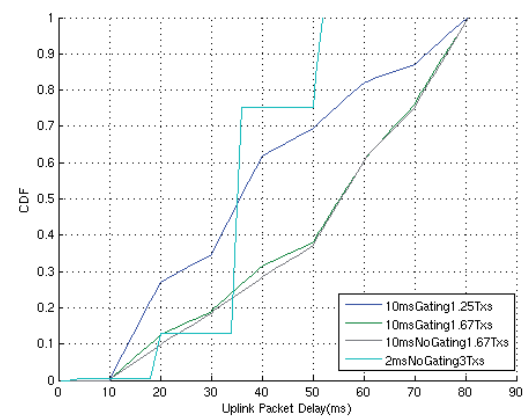

Fig. 2. Uplink packet delay distribution.

Besides, more HARQ transmissions, more delay VoIP packet would suffer. However, there is not much difference on the packet delay between gating $\mathrm{DPCCH}$ and continuous $\mathrm{DPCCH}$ with the same average transmission times.

\section{B. Performance of VoIP over HSDPA with the packet age aided scheduling}

Based on aforementioned uplink packet delay information, the downlink delay budget for each packet can be calculated. Then, this packet specific delay budget is used for the downlink scheduling. Fig. 3 depicts the cell outage level as a function of downlink user number per sector. It is noted that the static delay budget used in early studies, $80 \mathrm{~ms}$ and $150 \mathrm{~ms}$, can just provide the lower bound and upper bound of the downlink capacity. And $150 \mathrm{~ms}$ delay budget for only downlink air interface transmission is even not reasonable when the call is between mobiles. So the noticeable gain of the scheme with the packet specific delay budget can be observed compared to the case with the $80 \mathrm{~ms}$ fixed delay budget.

Shorter delay in the uplink can offer more time for the downlink scheduling which can exploit the relative better channel condition for the UE transmission. This will make the downlink transmission more efficiently, and then offer more

TABLE III.

SUMMARY OF THE UPLINK VOIP CAPACITY RESULTS

\begin{tabular}{|l|l|l|l|l|l|l|l|}
\hline & \multicolumn{5}{|c|}{ Capacity criteria: 5\% FER over 10 } & \multicolumn{2}{c|}{ Capacity criteria: Noise rise 6 dB } \\
\hline TTI & $\begin{array}{l}\text { Average number of } \\
\text { transmissions }\end{array}$ & $\begin{array}{l}\text { Continuous } \\
\text { DPCCH }\end{array}$ & $\begin{array}{l}\text { Gated } \\
\text { DPCCH }\end{array}$ & Gating Gain & $\begin{array}{l}\text { Continuous } \\
\text { DPCCH }\end{array}$ & $\begin{array}{l}\text { Gated } \\
\text { DPCCH }\end{array}$ & Gating Gain \\
\hline $2 \mathrm{~ms}$ & $\sim 3$ & 82 users & 123 users & $50 \%$ & 75 users & 106 users & $41 \%$ \\
\hline \multirow{2}{*}{$10 \mathrm{~ms} *$} & 1.25 & 65 users & 115 users & $77 \%$ & 61 users & 93 users & $52 \%$ \\
\cline { 2 - 7 } & 1.67 & 80 users & 120 users & $50 \%$ & 73 users & 103 users & $41 \%$ \\
\hline
\end{tabular}


UE leading an increase in the downlink capacity of up to $16 \%$ in the case of $2 \mathrm{~ms}$ TTI length compared to the static delay budget. Consequently, the simulation results are also quite in line with uplink packet delay results, i.e., the downlink capacity with the uplink packet transmission in $2 \mathrm{~ms}$ TTI is higher than the case with $10 \mathrm{~ms}$ TTI due to the shorter delay. It is also verified that DPCCH gating can improve the uplink performance significantly without sacrificing any downlink performance assuming the same transmission times for DPCCH gating and continuous DPCCH. Uplink HARQ transmission times would also affect the downlink performance because of its effect on the packet delay with this proposed scheduling.

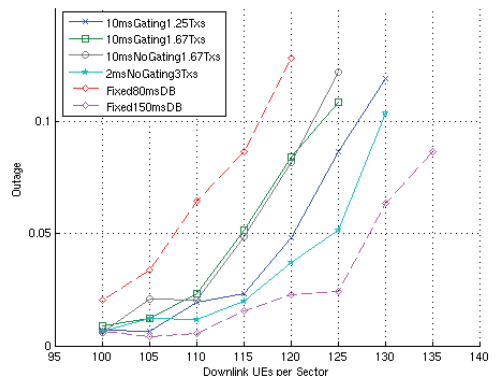

Fig. 3. Downlink VoIP capacity with different uplink transmission.

Hence, the packet specific delay budget can provide significant capacity gain in the downlink compared to the static delay budget. It can ensure the end-to-end performance but not only uplink or downlink performance. Assuming the uplink is from a land-line user, it is expected to provide more gain as shown in the case of $150 \mathrm{~ms}$, where the uplink transmission delay can be ignored.

C. Effect of uplink packet delay variation

In order to further investigate the uplink delay effect on the downlink capacity with the packet specific delay budget, the downlink delay budget is calculated based on a uplink packet delay of each VoIP call, which is sampled from a normal distribution function as shown in Fig. 4, where the average uplink packet delay is $80 \mathrm{~ms}$ and $90 \mathrm{~ms}$ with the standard deviation of 0,10 and 20 .

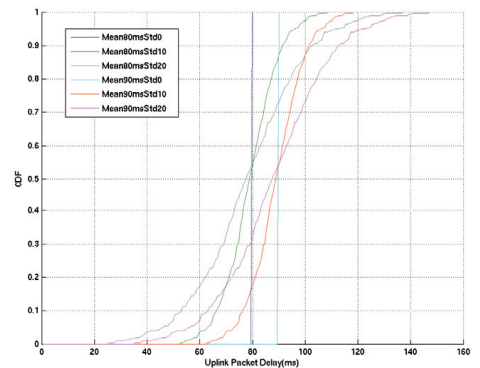

Fig. 4. Generated uplink packet delay distribution.

The downlink capacity results are shown in Fig. 5. It can be noted that larger standard deviation of uplink packet delay will lead to worse downlink performance at the same mean values. In some sense, the delay variation implies how burst the uplink VoIP packets arrive into the downlink scheduling buffer. The smaller variation means less bursty. This kind of bursty characteristic is mainly because the uplink air interface (re)transmission may result in uncertain packet delay and even disorder VoIP packets. However, the packet age aided scheduling can somehow relieve the pain from the bursty by prioritizing VoIP packets according to their ages.

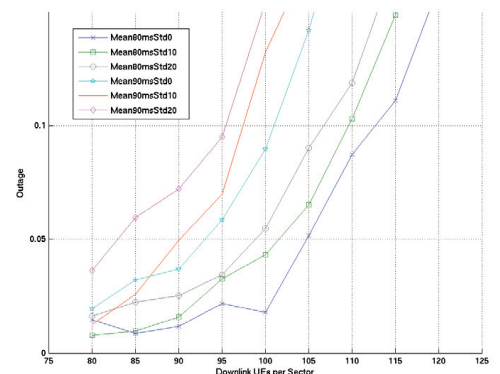

Fig. 5. Downlink VoIP capacity with generated uplink delay distribution

\section{CONCLUSION}

A packet age aided scheduling in HSDPA is proposed to improve the downlink VoIP capacity by utilizing the packet specific delay budget instead of the static one. The end-to-end system performance can be significantly improved by this way, especially for shorter uplink transmission delay. The results obtained from extensive system-level uplink and downlink simulations have shown that using the proposed scheduling can lead to an increase in the downlink cell capacity of up to $16 \%$. By applying the proposed method, the downlink performance can be improved considerably while the uplink performance remains the same, which can ensure the good end-to-end performance.

\section{References}

1] 3GPP TS 25.309, "FDD Enhanced Uplink; Overall description; Stage 2"

[2] 3GPP TR 25.896, "Feasibility Study for Enhanced Uplink (FDD)".

[3] 3GPP TS 25.308, "HSDPA; Overall description; Stage2".

[4] ITU-T Recommendation G.114, "One way transmission time"

[5] H.Holma, M.Kuusela, E. Malkamäki, K. Ranta-aho, Tao Chen, "VoIP over HSPA with 3GPP Release 7" PIMRC, Helsink, September 2006.

6] W.Bang,, K. I. Pedersen, T.E. Kolding, and P. E. Mogensen (2005), "Performance of VoIP on HSDPA", IEEE Proc. Ve-hicular Technology "Performance of VoIP on HSD

[7] Petteri Lunden, Markku Kuusela, "Enhancing Performance of VoIP over HSDPA". IEEE Proc. Vehicular Technology Conference, Spring, 2006.

[8] W.Richard Stevens, "TCP/IP Illustrated,Volume 1:The Protocols", Addison-Wesley, 1994, ISBN 0-201-63346-9

[9] R. Cuny, A. Lakaniemi, "VoIP in 3G Networks: An end-to-end quality of service analysis," IEEE, VTC spring, vol. 2, pp. 930--934, April 2003.

[10] 3GPP2 Technical Specification Group C, 3GPP2/TSG-C.1002: "1X EVDV Evaluation Methodology" 
RADIO ACCESS NETWORK VOIP OPTIMIZATION AND PERFORMANCE ON 3GPP HSPA/LTE

\author{
by \\ Kuusela, M., Chen, T., Lundén, P., Wang, H., Henttonen, T., Ojala, J., Malkamäki, E. \\ 2009 \\ Book Chapter 10 of VoIP handbook, "Applications, technologies, reliability and se- \\ curity", Page 319-363
}

Reproduced with kind permission by CRC press 
VI

\title{
DEVICE-TO-DEVICE COMMUNICATION FOR LTE-ADVANCED NETWORK SYSTEM
}

\author{
by \\ Chen, T., Kunnari, E. and Ristaniemi, T. 2014 \\ Report of the Department of Mathematical Information Technology, \\ University of Jyväskylä, ISBN 978-951-39-5919-7
}

Reproduced with kind permission by University of Jyväskylä 
Reports of the Department of Mathematical Information Technology Series D. Telecommunication

No. D 1/2014

Device-to-Device Communication for LTE-Advanced Network System

\author{
Tao Chen Esa Kunnari \\ Tapani Ristaniemi
}

\author{
University of Jyväskylä \\ Department of Mathematical Information Technology \\ P.O. Box 35 (Agora) \\ FI-40014 University of Jyväskylä \\ FINLAND \\ fax +358 142602771 \\ http://www.mit.jyu.fi
}


Copyright (c) 2014

Tao Chen and Esa Kunnari and Tapani Ristaniemi and University of Jyväskylä

ISBN 978-951-39-5919-7

ISSN 1456-4386 


\title{
Device-to-Device Communication for LTE-Advanced Network System
}

\author{
Tao Chen ${ }^{\dagger}$ Esa Kunnari ${ }^{\ddagger}$ Tapani Ristaniemi§
}

\begin{abstract}
Device-to-device (D2D) communication has been proposed as a mean for local connections in future cellular networks in order to provide improved throughput, spectrum savings, and longer battery lives. Here we widen the investigations of D2D communication to a 3GPP Long Term Evolution (LTE) system simulated in the interference limited macro-cellular environment, in which D2D communication is applied with D2D specific power control and resource allocation schemes and with a wide range of deployment ratios. Furthermore, we introduce channel model parameters applicable for a fair performance comparison between conventional cellular and D2D enhanced systems. The simulation results demonstrate that both the D2D and cellular users can gain significant throughput improvements when appropriate power control and resource allocation methods are used. This is further emphasized when the share of D2D users increases.
\end{abstract}

\section{Introduction}

It has been envisioned that new innovative local services will make up a significant part of traffic in future cellular systems. In these services, a client and a service provider would often be in the immediate vicinity of each other and much further away from a base station. Therefore, they could potentially have much better radio channel between themselves than towards a base station. However, the conventional cellular operation cannot handle their mutual traffic any differently but will transfer it via a base station and further route it through the core network. Similar suboptimal relaying of traffic through a base station and the core network occurs in a file share, voice call or other communication between two close-by

\footnotetext{
† Department of Mathematical Information Technology, University of Jyväskylä, P.O. Box 35 (Agora), FI-40014 University of Jyväskylä, Finland, sh inechen@gmail • com

$\ddagger$ Centre for Wireless Communications, University of Oulu, P.O.Box 4500, FI-90014 University of Oulu, Finland, esa.kunnarieee.oulu.fi

§ Department of Mathematical Information Technology, University of Jyväskylä, P.O. Box 35 (Agora), FI-40014 University of Jyväskylä, Finland, tapani.e.ristaniemi@j yu . fi
} 
users of conventional cellular systems. This twofold transmission of the same data, which takes place first in the uplink and then in the downlink, will result in a waste of scarce radio frequency spectrum and a higher end-to-end packet delay in comparison with a direct transmission between the two communicating devices. Meanwhile, it increases the burden of the core network for delivering and processing the duplicated data. Moreover, the possibly longer distances and, therefore, greater path losses of cellular links in comparison with that of a direct link will require much higher transmit powers, which in turn results in increased power consumption and intra-system interference.

In the literature, the direct communication between two terminals of an infrastructure-based network has been called with various names, out of which we use the device-to-device (D2D) term. Among the first propositions of using D2D communication in conjunction with cellular communication was the one in [1], where this hybrid scheme was considered to be especially suitable for applications such as communication between operational mobile robots and inter-vehicle communication. The latter usage of D2D communication has been an integral part of a number of multi-hop peer-to-peer network extensions proposed for the conventional cellular network topology. This network model was reviewed and its performance for Internet access was investigated in [2]. A survey on D2D communications in various infrastructure-based networks has been carried out in [3]. Network controlled D2D communication without the ad-hoc relaying functionality has also been proposed as an underlay to an IMT/LTE-Advanced cellular network and its performance has been simulated in a single-cell scenario and Manhattan grid model with indoor D2D communication [4] and in an indoor environment [5]. The optimal selection between the D2D and cellular modes within a WCDMA cellular system has been analyzed in [6]. Time hopping based radio resource allocation schemes for mitigating interference arising from the spatial reuse of cellular resources for D2D communication were proposed in [7]. In most of the prior studies, D2D concept is aiming for a generic solution without specific consideration on the practical constraints of LTE system. Furthermore, the D2D link distance has been either fixed or artificially limited, and the effect of the D2D user penetration ratio has not been considered. In addition, the channel and system models used have been fairly limited and highly simplified.

Recently, D2D is getting to be a hot topic with more and more interest from the academy and industry. In $[8,9,10]$, the general concepts and survey on the latest research outcomes have been presented. Service driven D2D communication are discussed in [11] and [12] considering the initial service setup procedure and content downloading service. More specifically, the various RRM schemes for D2D communications have been also presented and evaluated. For resource allocation, 
interference coordination based schemes are extensively studied in $[13,14,15,16$, 17]. In addition, game theory is also considered for resource allocation as indicated in [18]. For power control schemes, [19] and [20] proposed dynamic power control schemes for interference coordination. In [21], it provides some QoS-aware mode selection and resource allocation schemes. Random access protocol for collision avoidance in cellular device-to-device communication is presented in [22].

In this report, we present a D2D enhanced system concept with some essential radio resource management (RRM) schemes aiming for the long term evolution of 3GPP LTE system. Moreover, we extend the performance study of D2D communication within cellular networks to an LTE system under the interference limited micro-cell environment and the coverage limited macro-cell environment. To address the above mentioned shortcomings of the prior studies, we have evaluated the performance of this system with a 3GPP compliant multi-cell LTE network simulator that is complemented with D2D functionality based on our concept and an appropriate channel model. D2D communication system concept with its power control and resource scheduling alternatives in addition to D2D mode selection is presented in Section 2 and the adopted channel model in Section 3. Simulation results are presented in Section 4 and, finally, conclusions are drawn in Section 5.

\section{Device-to-Device Communication System}

In general, the D2D communication can be deployed either under LTE frequency division duplexing (FDD) cellular system by using the uplink cellular band for D2D communication or under LTE time division duplexing (TDD) cellular system by utilizing the uplink subframes of TDD cellular resources. Thanks to the high commonality between LTE FDD and LTE TDD system in the 3GPP system design, it provides the feasibility for such integrated FDD/TDD implementation at UE in terms of the cost and the complexity.

In this report, the considered D2D communication is assumed to use TDD and it takes place in the uplink band of an LTE FDD cellular system. With this setup a dual mode FDD/TDD user equipment (UE) can use the FDD mode for conventional cellular operation and an enhanced TDD mode for D2D communication. The uplink was chosen in order to ease out the control of the effects that the D2D communication has on the cellular operation. On the other hand, the interference situation is getting more complicated compared to the conventional cellular system due to the mix of the centralized cellular system and the distributed D2D communication system, which has to be addressed for the 
success of D2D communication under cellular system. Essentially, there are four types of interference:

1. $\mathrm{d} 2 \mathrm{c}$ interference: Interference from D2D transmission to the cellular link reception at eNB.

2. d2d interference: Cross-link interference between D2D communications.

3. c2c interference: Inter-cell interference between cellular transmissions.

4. c2d interference: Interference from cellular links to D2D Rx UE.

In addition, the interference can be further categorized as intra-cell and inter-cell interference. There is only c2c inter-cell interference assuming the orthogonal resource allocation for cellular communications in LTE system. However, intra-cell interference can be occurred for $\mathrm{d} 2 \mathrm{c}, \mathrm{d} 2 \mathrm{~d}$, and $\mathrm{c} 2 \mathrm{~d}$ cases in addition to the inter-cell interference, which depends on resource allocation schemes among D2D and cellular communications. For example, in case of frequency resource reusing, there will be additional intra-cell $\mathrm{d} 2 \mathrm{~d}, \mathrm{~d} 2 \mathrm{c}$ and $\mathrm{c} 2 \mathrm{~d}$ interference, especially for the case with imperfect spatial multiplexing.

The setup and the related interference couplings are further illustrated in Figure 1, where it is assumed the D2D users are allocated dedicated resources within a cell while the frequency reuse factor of the cellular network equals one, i.e., there is no intra-cell D2D or cellular interference similarly to the conventional LTE system. Besides, the shared resource allocation for spatial reuse is also allowed to further improve the spectrum usage as long as there is sufficient decoupling among D2D users or between D2D user and cellular users, in which the occurred intra-cell interference from D2D users has to be well controlled for achieving the performance gain. 


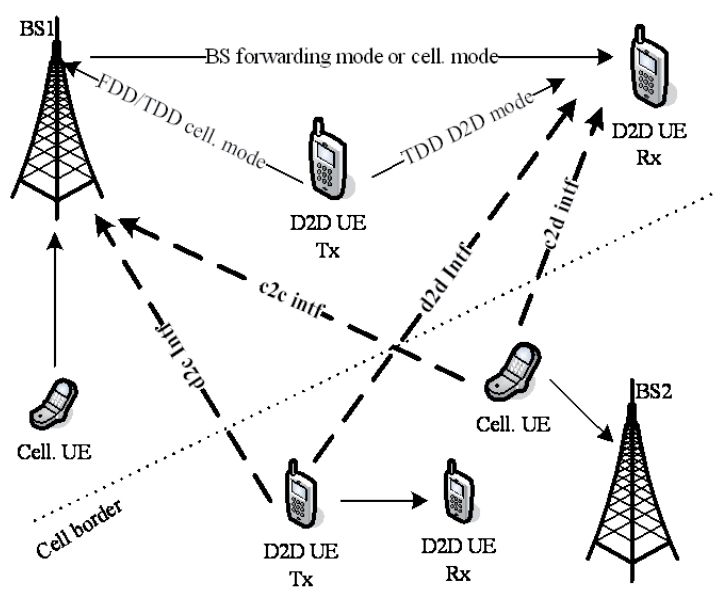

Figure 1: D2D and cellular links (solid line) and interference couplings (dashed line) when D2D operation uses the uplink band of a cellular system with the frequency reuse factor of one and dedicated D2D resources within a cell.

The Signal-to-Interference-Noise-Ratio (SINR) at a D2D Rx UE per subcarrier for D2D communication can be expressed as

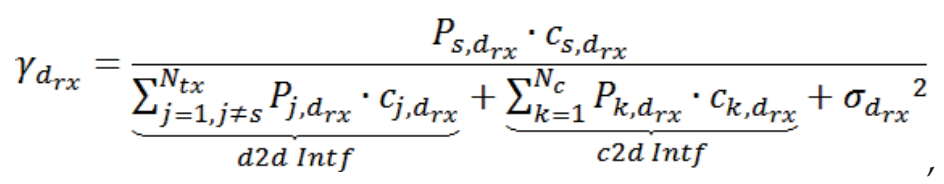

and the perceived SINR at eNB for cellular uplink reception is

$$
\gamma_{b}=\frac{P_{s, b} \cdot c_{s, b}}{\underbrace{\sum_{j=1}^{N_{t x} P_{j, b} \cdot c_{j, b}}}_{d 2 c \text { Int } f}+\underbrace{\sum_{k=1}^{N_{c}} P_{k, b} \cdot c_{k, b}}_{c 2 c \text { Intf }}+\sigma_{b}^{2}},
$$

where $P_{i, j}$ and $c_{i, j}$ denote the transmit power and the channel gain for the link between device $i$ and $i$ respectively; $s, d_{r x}$ and $b$ stand for the Tx UE, D2D Rx UE and base station; $N_{t x}$ is the number of D2D Tx UEs and $N_{c}$ is the number of cellular UEs; ${\sigma_{d_{r x}}}^{2}$ and $\sigma_{b}{ }^{2}$ represent additive white Gaussian noise at D2D Rx UE receiver and base station receiver separately.

According to SINR expression given in equations (1) and (2), it can be noted that the overall system performance in a mixed system with both D2D and cellular communications can be affected by several factors:

- D2D pathloss with the impact on D2D mode selection criteria,

- D2D transmit power control scheme, 
- Frequency resource allocation strategy,

- Proportion of D2D communication.

These factors are also tightly coupled between each other, which makes a big challenge for system operation and optimization.

In detail, the key components of the concept: the selection between the D2D and cellular operation modes, the power control of D2D links, and the allocation of D2D radio resources, are discussed in Sections 2.1, 2.2, and 2.3, respectively.

\subsection{Mode Selection}

A pair of close-by cellular users supporting D2D operation can communicate by using the conventional cellular mode, the simplified cellular mode or D2D mode of operation. The simplified cellular mode is an optimization of the conventional cellular mode based on packet direct forwarding or routing via eNB rather than the gateway, which can significantly reduce the end-to-end packet delivery latency. In short, the latency can be expressed below for the conventional cellular mode $\left(T_{c c}\right)$, the simplified cellular mode $\left(T_{s c}\right)$ and the direct D2D cellular mode $\left(T_{d 2 d}\right)$ :

$$
\begin{aligned}
& T_{c c}=t_{u l}+t_{c n}+t_{d l}, \\
& T_{s c}=t_{u l}+t_{d l,} \\
& T_{d 2 d} \approx t_{u l},
\end{aligned}
$$

where $t_{u l}$ and $t_{d l}$ denote the delay for the successful packet delivery in uplink and downlink air interface separately including the Hybrid automatic repeat request (HARQ) (re)transmission delay and UE/eNB processing delay; $t_{c n}$ stands for the routing delay for the packet in the core network. It is clear that the simplified cellular mode based on packet routing or direct forwarding via layer 2 function can avoid the huge latency in the core network caused by the conventional cellular mode using layer 3 routing (up to 200ms delay reduction) whereas the direct D2D transmission mode can further reduce the half latency thanks to one-hop instead of two-hop transmission in the simplified cellular mode. Considering the fact the devices with the potential for D2D communication are typically close to each other and under the same eNB, the simplified cellular mode can be exploited as one mode to offer the D2D service.

To maximize the system throughput, the optimal criterion for selecting between these two modes would be the sum-rate criterion. That is, the mode which would result in the highest system sum-rate would get selected. For optimality, the mode selection should also be done jointly with power control and resource allocation based on instantaneous channel and interference conditions. This, however, would largely increase complexity and control signaling. Depending on the phase of D2D 
operation, Initial D2D link setup phase or D2D operation maintenance phase, the mode selection criteria can be different considering the tradeoff between the performance and link management complexity.

In the initial D2D link setup phase, the mode selection can be based on a longterm measurement, e.g., pathloss or the received power. The purpose is to establish a stable communication link for initial D2D setup. During this phase, eNB is lacking of the full knowledge of link quality due to limitation on channel information reporting. In this case, we resort to a suboptimal but much simpler and more practical approach where the mode selection is done based on the received power level.

The D2D mode is selected if the smaller of the powers received at the two directions of a D2D link between two users would be higher than the smaller of the two powers that a base station would receive from these two users. Otherwise the users operate in the cellular mode. The above criterion can be also expressed as

$$
\min \left(P_{1,2, d_{r x}} \cdot c_{1,2}, P_{2,1} \cdot c_{1,2}\right)>\min \left(P_{1, b} \cdot c_{1, b}, P_{2, b} \cdot c_{2, b}\right),
$$

where $P_{i, j} \cdot c_{i, j}$ is the power from unit $j$ that is received at unit $i$ and unit index $b$ belongs to the base station and indices one and two to the D2D users. With this criterion we assume that the bidirectional cellular link between the users is limited by the uplink and that the users may have different transmit powers also in D2D operation. Note that if the transmit powers of the links considered in (6) are the same, this criterion reduces to selecting the mode with the lowest path loss.

Although the above simple suboptimal mode selection criterion ensures that the mode and link with the highest signal level is chosen, it does not take into account the interference level at the receiver. In particular, the presence of strong inter-cell interference variation during the operation at the cell edge may eventually lead to degraded performance in the D2D mode. However, during D2D operation phase with the established D2D link and the simplified cellular link, eNB can make a fast or dynamic mode selection based on the frequent channel quality indication (CQI) report which can capture the effect of varying interference. In this case, the criteria can be established based on the short-term D2D SINRs $\left(\gamma_{2,1}\right.$ and $\left.\gamma_{1,2}\right)$ derived from the reported D2D CQIs, and cellular uplink SINRs $\left(\gamma_{1, b}\right.$ and $\left.\gamma_{2, b}\right)$ estimated from the uplink sounding reference signal (SRS). Similarly to (6), the SINR based criteria can be expressed as

$$
\min \left(\gamma_{2,1}, \gamma_{1,2}\right)>\min \left(\gamma_{1, b}, \gamma_{2, b}\right) .
$$

In practical operation, the dynamic mode switching would require the unique sequence number for the packet by D2D Tx UE so that the D2D Rx UE can re-order the packets received from either eNB forwarding or D2D direct transmission, as 
indicated in Figure 1. The dynamic mode switching can be transparent to the D2D UEs due to the characteristics of broadcasting transmission. Whether to dynamically switch is fully controlled by eNB via the resource allocation grant.

One use case for dynamic mode switching is illustrated in Figure 2. In this case, based on the established D2D communication eNB can derive the SINRs for two modes based on D2D CQI reports and SRS estimation. D2D Tx UE will transmit the data to Rx UE using the resources indicated by the D2D resource grant from eNB. Meanwhile, the packets can also be received by eNB, given the suitable transmit power setting. In retransmission phase triggered by $\mathrm{D} 2 \mathrm{D}$ negativeacknowledge (NACK), eNB can choose whether to retransmit the data by eNB forwarding mode or D2D direct mode supposing the packet has been received by eNB successfully in the initial transmission. In case of eNB forwarding mode, eNB will suspend the resource allocation to D2D Tx UE for retransmission. Instead, eNB would retransmit the data directly to D2D Rx UE. For each retransmission, eNB can dynamically determine the mode of communication based on the estimated SINRs. eNB can resume the D2D transmission via a D2D resource grant for either new transmission or retransmission. The dynamic mode switching can secure the robust and flexible communication between two devices, especially in case of varying channel and interference conditions. In addition, it can also reduce the retransmission delay since there is no need of D2D grant with UE processing (up to $3 \mathrm{~ms}$ delay) compared to eNB direct forwarding mode.

Besides, it should be kept in mind that there is another benefit of downlink resource saving with direct D2D communication only using the uplink resources, compared to the simplified or conventional cellular transmission mode where BS has to transmit the same data to the peer user using the downlink resources. Essentially, this also provides the potential for D2D communication triggered by offloading the traffic in cellular downlink for the scarce downlink resources. 


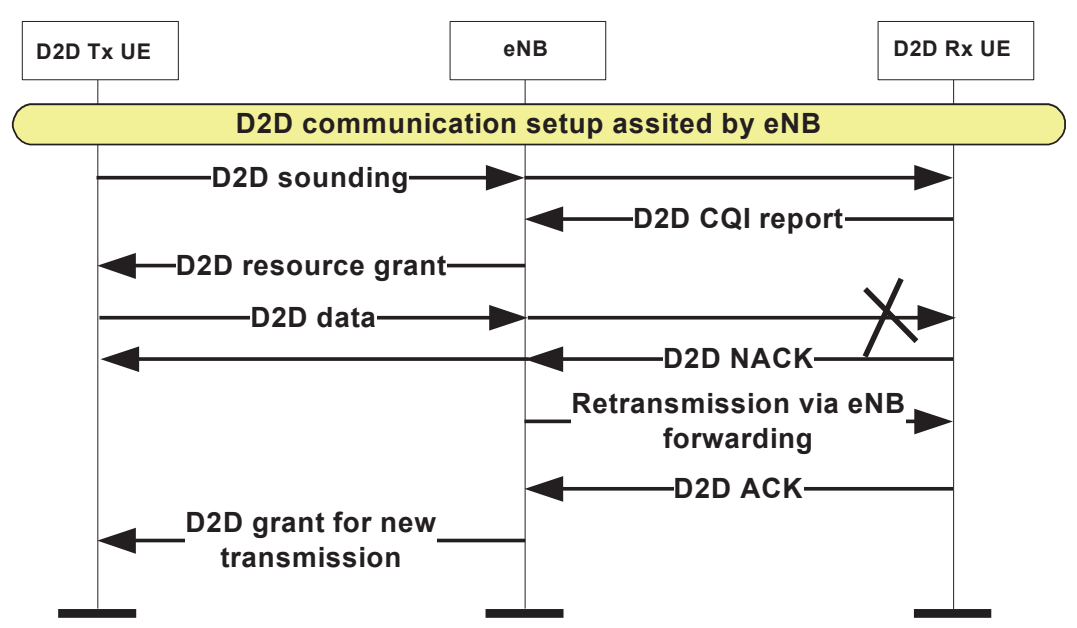

Figure 2: Illustration of sequence diagram for dynamic mode switching.

Thus, there can be different criteria for the mode selection depending on the target of network optimization. The key for However, the interference caused to and received from the other users in the D2D mode can be affected to some extent by power control and resource allocation scheme, which would be further discussed in the following sections. For simplicity, the term "cellular mode" would be used to represent the simplified cellular mode below.

\subsection{Power Control}

Both the cellular and D2D links use the fractional power control according to the LTE specifications [25]. For the D2D links, we consider two schemes which differ in what path loss is to be fractionally compensated. The first scheme targets the path loss in the D2D link, whereas the second scheme uses the loss of the cellular link towards the serving base station. In principle, the transmit power for D2D communication in subframe $i$ can be determined by

$$
P_{d 2 d}(i)=\min \left(P_{c}(i), P_{0}+\alpha \cdot P L+10 \cdot \log _{10}(M(i)),\right.
$$

where $P L$ is the pathloss for either cellular link or D2D link depending on the PC scheme to be discussed later, $P_{c}(i)$ is the transmit power assuming the cellular mode operation in subframe $i$, which is derived based on the cellular link pathloss for $P L$ in (2), and $P_{0}$ is the power per PRB, $M(i)$ is the allocated bandwidth in subframe $i$ for data transmission measured in number of physical resource blocks (PRBs) specified in [25], $\alpha$ is for partial pathloss compensation with a value in between 0 and 1. Specifically, supposing $\alpha=1, P_{0}$ can be interpreted as the target received power per PRB to achieve a certain SNR. 
Essentially, the D2D link based power control of D2D users operates in the same principle as the power control of cellular users, but due to the shorter average link distances of D2D users with the proper mode selection, their transmit power levels are much lower. This saves D2D users' battery resources and decreases their interference towards other users in comparison with their operation in the cellular mode. On the other hand, the low D2D link power without consideration of the interference may be overshadowed by nearby cellular users, especially in the cell edge where the transmit powers of the neighbor cellular users are at the highest. This disadvantage can be countered by the cellular link based power control that allows the user to transmit in the D2D link with the same power as it would if it was transmitting to the base station. This power also serves as the upper limit for the D2D link based power control, which implies that D2D transmission won't cause more interference to the incumbent cellular users than the conventional cellular mode. With the cellular link based power control option, however, the D2D transmission does generate more interference to the system, but not more as it would in the cellular mode. Thus, a sensible D2D PC scheme can effectively reduce the power consumption and/or enhance the overall system performance.

\subsection{Resource Allocation}

The primary radio resource allocation scheme we consider for D2D links in this study is similar to that of conventional cellular users. That is, D2D pairs are scheduled dedicated resources by their serving base station in the same way as the base station schedules its cellular users. Moreover, the D2D links have access to all the same time and frequency resources as the cellular links. Both link types are scheduled by using the proportional fair scheduling algorithm and are assigned the same number of PRBs. The number of users and the number of PRBs per user were set with the view to having a fully loaded system while having an activity factor of one for all users.

While the above centralized resource allocation scheme with dedicated resources avoids intra-cell interference, it is not able to utilize the spatial reuse gain potential of D2D communication. To explore this potential, we considered also a decentralized resource allocation scheme where the D2D pairs employ autonomous scheduling. In that case, the D2D pairs use the resources providing the highest scheduling metric for their link. This may result in a blind reuse of resources scheduled to cellular or other D2D users in the cell. The spatial reuse of the resources within a cell can increase the spectral efficiency if the resulting intracell interference can be either tolerated, avoided, or suppressed. If not, the intra-cell interference may eventually degrade the spectral efficiency. In addition, the available radio resources are increased for both the cellular and D2D users in 
comparison with our primary resource allocation scheme ensuring dedicated resources. This provides a higher frequency diversity gain, which becomes beneficial especially in light system loads where there are less collision between the resources allocated to the cellular and D2D users. One more benefit for the autonomous reuse is the low signaling overhead in the practical operation. However, in case of the high load, the gain of spatial reuse may rely on more precise interference control for decoupling of the shared users. Then, the network controlled reuse scheme for resource allocation associated with mode selection and power control may be more favored. So, selection of the suitable resource allocation scheme can further improve the spectrum usage.

\section{D2D Channel Model}

Since the interest in D2D communications within a cellular system has raised relatively recently, there are no widely accepted channel models for D2D links with cellular radio frequencies. Furthermore, models that would encompass comparable modeling of both the D2D links (MS-MS) and the conventional cellular links (MS-BS) are even scarcer. One existing study that includes channel models for both the MS-BS and MS-MS links, as well as for links involving relays was presented in [23]. From those results, we extracted parameters for the path loss and LOS probability discussed in Sections 3.1 and 3.2, respectively.

\subsection{Path Loss Model}

The mean path loss of a link can be expressed by the well-known log-distance model:

$$
L[d B]=L_{0}+10 n \log 10\left(d / d_{0}\right)+20 \log 10(f / M H z),
$$

where $L_{0}$ is the path loss at the reference distance $d_{0}, n$ is the path loss exponent, $d$ is the link distance, and $f$ is the carrier frequency. The adopted values for $n$ and $L_{0}$ with one meter reference distance $d_{0}$ and $2 \mathrm{GHz}$ carrier frequency $\mathrm{f}$ that were given for both the line-of-sight (LOS) and non-LOS (NLOS) type MS-BS and MS-MS links in [23] are represented in Table 1. With $n=2$, the LOS path loss for both the MS-BS and MS-MS links is simply the free-space path loss. This, together with the relatively high path loss exponents for NLOS links ensures that both the strong desired and interfering links with longer distances as well as the relatively heavily attenuated links with shorter distances will be well represented. 
Table 1: Path Loss Parameters

\begin{tabular}{ccc}
\hline \hline Link type & $L_{0}$ & $n$ \\
\hline LOS MS-BS/MS & -27.6 & 2 \\
NLOS MS-BS & -57.37 & 4.88 \\
NLOS MS-MS & -62.01 & 5.86 \\
\hline \hline
\end{tabular}

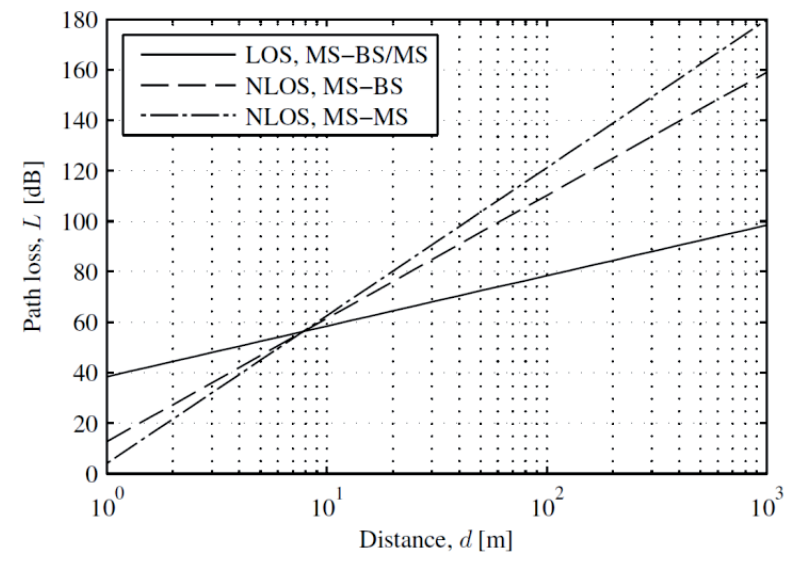

Figure 3: Path loss as a function of link distance for LOS and NLOS links between a base station and a mobile or between two mobiles.

The path losses obtained with the above parameters are illustrated in Figure 3. It can be seen that for very short link distances, which are below the shortest distances at which the models have been fitted to experimental data, the proportions between the path losses of different link types become illogical. This behavior is typical for log-distance path loss models and was avoided here by lower bounding the path loss to the free-space path loss that is now equal to the LOS path loss. In any case, these shortest links are very likely to be LOS links, as will appear in the following.

\subsection{Line-of-Sight Probability}

The link distance dependent LOS probabilities were derived by fitting a LOS probability equation similar to the equation given for mobile station to relay station (MS-RS) links in [24] to the LOS probability illustrations in [23] based on visual fit. The considered LOS probability equation includes two exponential terms, one of which defines the divergence of the probability from unity at short link distances, and the other of which determines the convergence of the probability 
towards zero at long link distances. We elaborated this equation further by parameterizing it as:

$$
P_{\text {LOS }}=1-P_{j}-\min \left[1-P_{j}, k_{1} \exp \left(-\frac{d_{1}}{d}\right)\right]+\min \left[1-P_{j}, k_{0} \exp \left(-\frac{d}{d_{0}}\right)\right],
$$

where $P_{j}$ is interpreted as the joint point of the exponential terms, $d_{1}$ and $d_{01}$ determine the distances at which the terms diverge from unity and converge towards zero, and $k_{1}$ and $k_{0}$ contribute to the slopes of the terms, respectively. The above parameters obtained for MS-BS and MS-MS links are given in Table 2, and the resulting LOS probabilities are illustrated in Figure 4.

Table 2: Loss Probability Parameters

\begin{tabular}{cccccc}
\hline \hline Link & $P_{j}$ & $k_{1}$ & $d_{1}$ & $k_{0}$ & $d_{0}$ \\
\hline MS-BS & 0.85 & 6 & 130 & 1.6 & 56 \\
MS-MS & 0.80 & 7 & 130 & 2.0 & 40 \\
\hline \hline
\end{tabular}

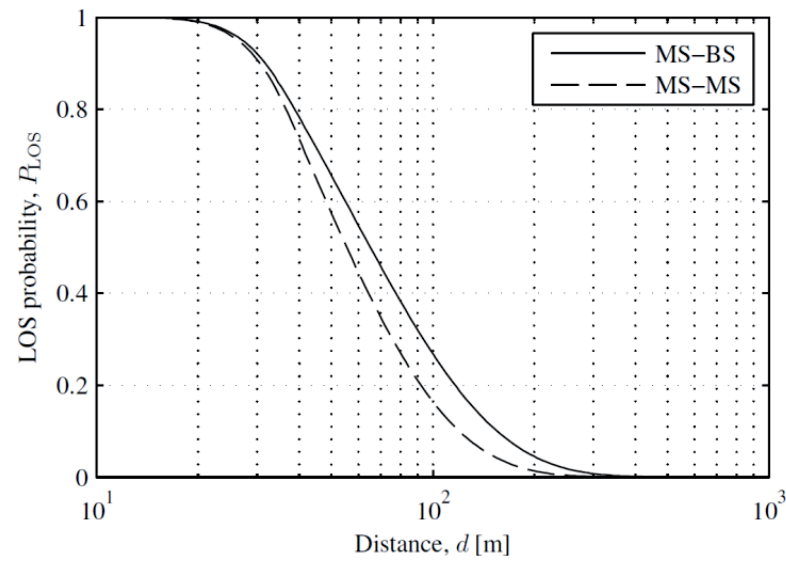

Figure 4: LOS probability as a function of link distance between a base station and a mobile or between two mobiles. 


\section{SIMULATION SETUP AND RESULTS}

The main simulation and LTE system parameters, which are either alternative or differ from those defined in LTE specifications, are given in Table 3. If the location of a D2D Rx user dropped into the network layout did not led up to the D2D operation mode, the user was relocated until the D2D mode got chosen. This procedure is similar to the widely used load balancing approach that was also used here for the cellular and D2D Rx users. These procedures enable to study the effects of D2D communication with a fixed number and proportion of cellular and D2D users per cell. Without loss of generality, we assume the traffic in D2D links to be unidirectional within a simulation drop.

The cellular layout is a wrapped-around hexagonal grid with 19 base station sites and 3 sectors per site. Both the interference limited scenario (3GPP Case 1) and the cover limited scenario (3GPP Case 3) as defined in [24] are adopted for an extensive performance evaluation, in which the inter-site distance (ISD) between eNBs is $500 \mathrm{~m}$ corresponding to an interference limited macro-cell scenario and $1700 \mathrm{~m}$ for a coverage limited macro-cell scenario. There is a balanced load of $N_{c}$ cellular users and $N_{t x}$ D2D Tx users per sector, that is, altogether $N_{c}+N_{t x}$ Tx users per sector. The total number of Tx users is fixed with 8 users in the study with a varying number of $N_{t x}$. The cellular users and D2D Rx users have random locations uniformly distributed over the whole network under the load balancing condition. Each of the D2D Tx users is given a random uniformly distributed location over a disc with a given maximum radius $r_{\max }$ around its pairing D2D Rx user. The value of $r_{\max }$ was set to be $400 \mathrm{~m}$, which is well above the maximum MSBS distance of $500 / \sqrt{3} \approx 289 \mathrm{~m}$ in Case 1 scenario, in order not to artificially limit the D2D link distances. There is no lower limit for the distance between mobiles, whereas for the MS-BS distance the limit is $35 \mathrm{~m}$.

The mean physical-layer user throughput with overhead for different number of D2D Tx users out of the total number of eight links per cell is illustrated with different cases in Figures 5 and 7 for the interference limited and the coverage limited scenarios separately. "Case 1" and "Case 3" are the baselines without any D2D operation. In both figures, the D2D pairs use dedicated resources in the without intra-cell interference from the other D2D and cellular users unless "Bld" is presented in the name of the case, while they use the D2D link based power control for "Dpc" cases and use the cellular link based counterpart for "Cpc" cases. In "Bld" cases, the D2D pairs use now the autonomous scheduling scheme, whereas their power control method is again the D2D link based one in order to limit the interference towards cellular users whose resources can be reused. These cases are based on the decentralized scheduling with independent resource 
allocation by D2D pairs and eNB separately, which is used to check the potential of D2D autonomous communication performance in case of lacking the network assistance.

Table 3: System Parameters

\begin{tabular}{ll}
\hline \hline Parameter & Value \\
\hline User deployment & Total 8 D2D/cellular Tx users per cell \\
$N_{t x}$ & $0,2,4,6,8 \mathrm{D} 2 \mathrm{D}$ Tx users \\
Macro cell deployment & 3GPP Case 1 with 500m ISD; \\
& 3GPP Case 3 with 1732m ISD \\
Minimum MS-BS distance & $35 \mathrm{~m}$ \\
Carrier frequency & $2 \mathrm{GHz}$ \\
Bandwidth & $10 \mathrm{MHz}$ \\
Power control & $P_{0}=-56 \mathrm{dBm}, \alpha=0.6$ \\
Modulation scheme & BPSK, QPSK, 16-QAM, 64-QAM \\
Coding rate & $1 / 3,1 / 2,2 / 3,3 / 4$ \\
BLER target & $20 \%$ after 1 st transmission \\
Max. retransmissions & 3 \\
Channel estimation & Non-ideal \\
Traffic model & Full buffer \\
Scheduler & Proportional fair \\
Resource allocation & 6 PRBs per Tx user \\
UE transmit power & 24 dBm \\
\hline \hline
\end{tabular}

\subsection{Performance for the interference limited scenario}

It can be seen from Figure 5 for the interference limited scenario that when the system operation shifts from cellular to D2D connections for "Dpc" cases, the mean throughput of the all users gradually increases with D2D share of the total system load as the inter-cell interference level decreases due to D2D lower transmit powers. For the same reason, the cell edge performance at $5 \%$-tile also benefits as the load shifts from cellular to D2D links with less inter-cell interference. For "Cpc" cases, both the mean throughput and cell edge throughput over all users gradually increases with D2D share of the total system load. However, the cell edge performance is significantly improved for "Dpc" cases than "Cpc" cases thanks to the low transmit power. On the other hand, the relatively low transmit power in "Dpc" case may result in the slightly lower mean throughput than the "Cpc" cases as the power setting based on the D2D pathloss doesn't take into account any intercell co-channel interference. In this interference limited scenario, the performance 
is more sensitive to the setting of the transmit power which may increase the interference while enhancing the signal strength. Essentially, the gain mechanism is quite different for "Dpc" cases and "Cpc" cases although both of them can improve the overall system performance. In practical operation, the selection of power control scheme may also depend on the goal of network optimization.

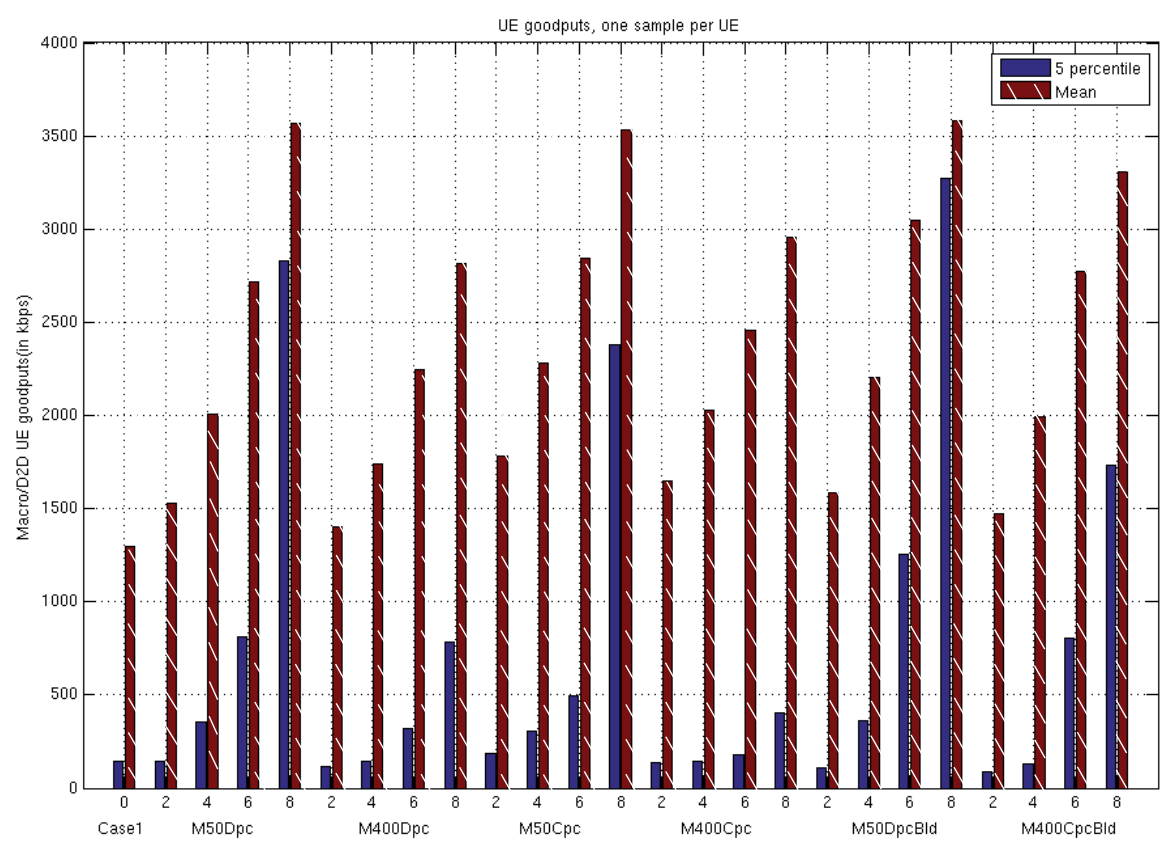

Figure 5: Performance results for deployment under Case 1 macro-cell scenario. 


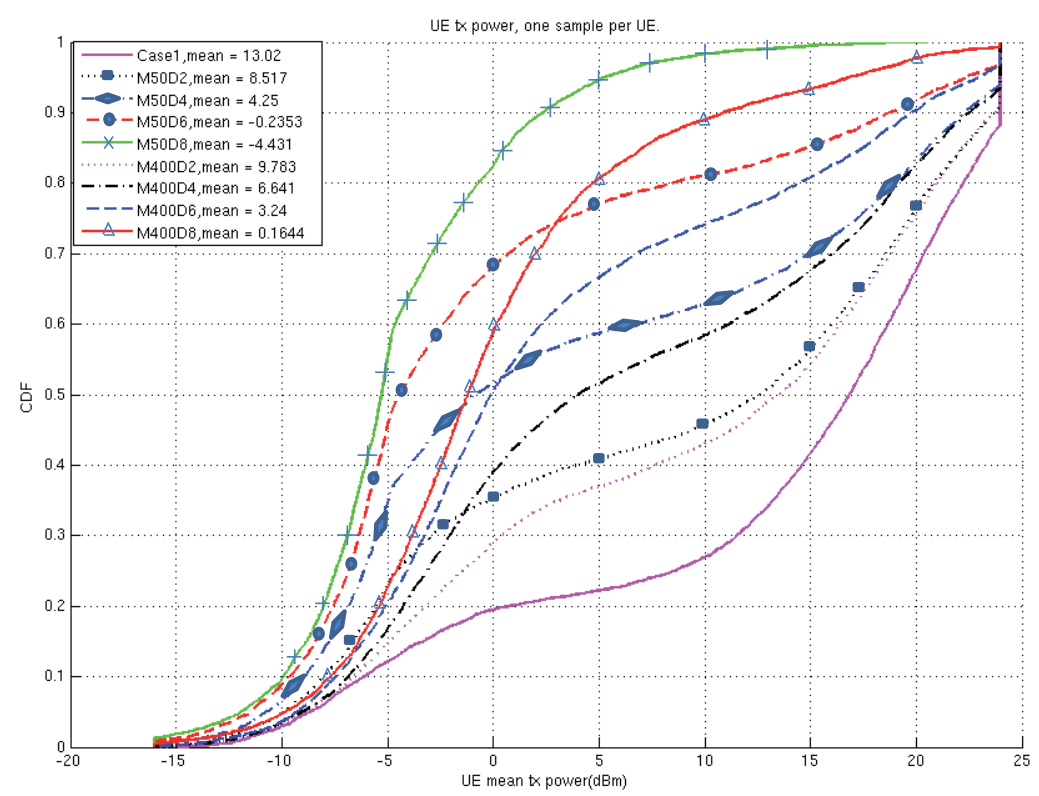

Figure 6. UE transmit power for deployment under Case 1 macro-cell scenario.

For the "Bld" cases with D2D autonomous scheduling, they show quite promising gains in terms of both the mean throughput and the cell edge throughput. Even though the resource allocation between D2D and cellular links is not fully orthogonal compared to the cases with centralized scheduling, it would not cause a big problem in case of the fractional load, especially considering the potential gain from spatial multiplexing between D2D and cellular links. Furthermore, the frequency diversity gain can be achieved thanks to frequency domain scheduling over the whole bandwidth for D2D users and cellular users separately, which can avoid the performance loss due to the shared frequency resources in the centralized scheduling.

In terms of UE Tx power consumption as shown in Figure 6, it is obvious that "Dpc" cases can significant save UE power whereas "Cpc" cases may drain the UE batter more quickly. With the shorter D2D distance and more D2D pairs, the mean power is getting much lower.

\subsection{Performance for the coverage limited scenario}

Similarly to the performance in the interference limited scenario, both the mean throughput and the cell edge throughput over all UEs are significantly improved 
compared to the baseline without any D2D operation thanks to the contribution of D2D operation, as indicated by Figure 7. In addition, it seems no clear performance difference for the cases with the maximum D2D distance of $50 \mathrm{~m}$. And for the case with maximum $400 \mathrm{~m}$ D2D distance, the overall performance is much lower than the case of D2D operation with maximum 50m distance. The larger allowed D2D operation range would require much higher transmit power as proved in Figure 8. In other words, in this coverage limited scenario with the larger D2D operation range, the overall performance is more limited by the affordable transmit power to improve the signal strength rather than the co-channel interference.

In 3GPP case 3, the inter-site distance is $1732 \mathrm{~m}$, which is much larger than Case 1. It will implicitly lower the UE density given the same number of UEs. Accordingly, the main source of the interference for D2D communication, c2d interference, can be reduced, which will potentially improve the overall performance. The mode selection coupling with the max D2D distance of $50 \mathrm{~m}$ would make the D2D performance quite good so that the 5\% UE throughput in the combined performance figure with mixed UEs is actually not from the D2D UEs but mainly from the cellular UEs. That's why the mean UE throughput in the combined figure is improved much more than 5\% UE throughput.

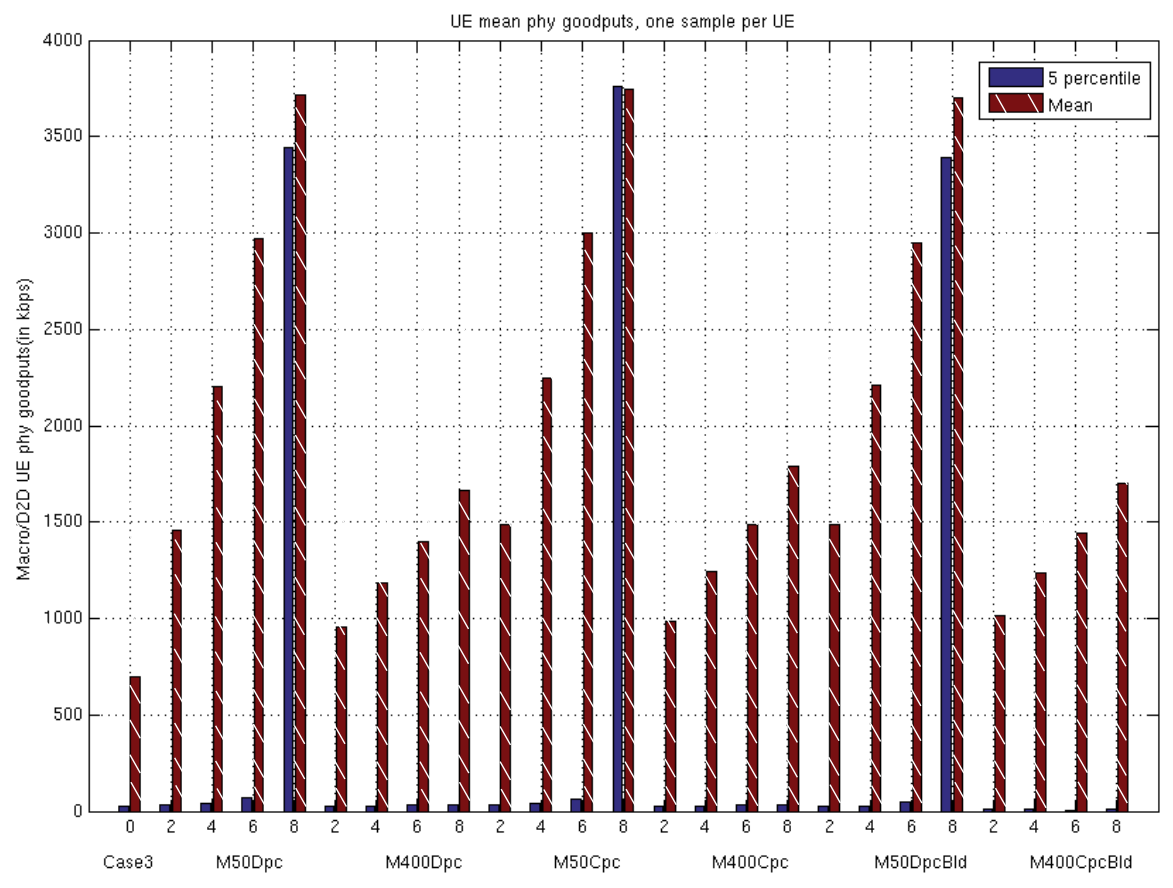

Figure 7: Performance results for deployment under Case 3 macro-cell scenario. 




Figure 8: UE transmit power for deployment under Case 3 macro-cell scenario.

And with D2D links only, there is no notable difference between the two PC schemes. This is because the inter-cell interference is more contributed by D2D transmission rather than the cellular transmission. Then the high transmit power with cellular link based PC would likely lead to more interference, thus slightly or even no improvement on the performance compared to D2D link based PC. For the throughput of cellular links, it should be kept the same thanks to the same interference level caused by the unchanged transmit power for D2D mode and cellular mode with cellular link based PC. Eventually, the overall performance is improved significantly.

As can be seen from the results for the autonomous D2D scheduling with D2D link based PC and maximum 50m D2D distance, the throughput performance is similar to those obtained with the same power control scheme but dedicated resources. However, in case of maximum 400m D2D distance, the autonomous scheduling may provide a slightly worse performance due to more severe crosslink interference caused by the larger D2D operation range with non-orthogonal resource allocation. In addition, compared to the performance with cellular link based PC, there is a slightly performance loss due to the same reason that there is no power control margin for D2D link based PC to overcome the unexpected intercell interference. Even though the autonomous D2D scheduling results in a blind reuse of resources, the benefits of the spectral reuse surpass the increased intra-cell 
interference. D2D pairs benefit from the full freedom of scheduling themselves on the best frequency resources, while the increased share of D2D users decreases the competition for schedulable resources among the remaining cellular mode users. Supposing there is no limitation of 6PRBs for resource allocation in this study, it is expected to further improve the overall performance with more frequency resources for reuse by each user.

Similarly to Case 1 in terms of power consumption, there is also a significant power saving with D2D link based power control. However, it can be noted that the maximum power is more reached in this case due to the larger communication range.

\section{Conclusion}

In this paper, we presented a system concept of D2D communication for the long term evolution of 3GPP LTE-Advanced network system to address the fast increasing local traffic. The performance of direct D2D communication in an LTE system over an interference limited micro-cellular environment and a coverage limited macro-cellular environment was evaluated. The D2D operation deployed the uplink band of the LTE FDD mode and took place between two same-cell users that had higher received power in their mutual link than in those towards the base station. The simulation results showed that besides removing the duplicated traffic from the downlink with additional downlink resource saving, D2D transmission can improve the overall system throughput also in the uplink. Since receiving D2D users are susceptible to interference from nearby high-power users that are scheduled on the same resources, their throughput can be greatly improved by allowing them to use higher cellular link based transmit power instead of D2D link based power when the majority of other users in the network are conventional cellular users. The lower D2D transmit powers, on the other hand, reduce the interference level and, therefore, improved the throughput of cellular users as the system load shifts from cellular to D2D links. Finally, the reuse of cellular resources for D2D communication, even when done so blindly, was demonstrated to provide further improvement in the system throughput.

\section{References}

[1] T. Adachi and M. Nakagawa, "A study on channel usage in a cellular-adhoc united communication system for operational robots," IEICE Trans. Commun., E81-B(7):1500-1507, 1998. 
[2] H.-Y. Hsieh and R. Sivakumar, "On using peer-to-peer communication in cellular wireless data networks," IEEE Trans. Mobile Comput., 3(1): 57-72, 2004.

[3] J. Lehtomäki, I. Suliman, J. Vartiainen, M. Bennis, A. Taparugssanagorn, and K. Umebayashi, "Direct communication between terminals in infrastructure based networks," in Proc. ICT Mobile and Wireless Commun. Summit, Stockholm, Sweden, Jun. 2008.

[4] P. Jänis, C.-H. Yu, K. Doppler, C. Ribeiro, C. Wijting, K. Hugl, O. Tirkkonen, and V. Koivunen, "Device-to-device communication underlaying cellular communications systems," Int. J. Commun. Netw. Syst. Sci., 2(3):169-178, 2009.

[5] K. Doppler, M. Rinne, C. Wijting, C. Ribeiro, and K. Hugl, "Device-to-device communication as an underlay to LTE-advanced networks," IEEE Commun. Mag., 47(12):42-49, 2009.

[6] S. Hakola, T. Chen, J. Lehtomäki, and T. Koskela, "Device-to-device (D2D) communication in cellular network - Performance analysis of optimum and practical communication mode selection," in Proc. IEEE Wireless Commun. and Networking Conf., Sydney, Australia, Apr. 2010.

[7] T. Chen, G. Charbit, and S. Hakola, "Time hopping for device-to-device communication in LTE cellular system," in Proc. IEEE Wireless Commun. and Networking Conf., Sydney, Australia, Apr. 2010.

[8] S. Vasudevan, K. Sivanesan, S. Kanugovi, and J. Zou, "Enabling data offload and proximity services using device to device communication over licensed cellular spectrum with infrastructure control", IEEE 78th Vehicular Technology Conference (VTC Fall), 2013

[9] A. Asadi, Q. Wang, and V. Mancuso, "A survey on device-to-device communication in cellular networks", IEEE Communications Surveys \& Tutorials, 2014, to appear

[10] M. Alam, D. Yang, J. Rodriguez, and R. Abd-Alhameed, "Secure device-todevice communication in LTE-A", IEEE Communications Magazine, 52(4):6673, 2014

[11] J. Lee, J. Gu, S. J. Bae, and M. Chung, "A session setup mechanism based on selective scanning for device-to-device communication in cellular networks", 17th Asia-Pacific Conference on Communications (APCC), 2011.

[12] Y. Li, Z. Wang, D. Jin, and S. Chen, "Optimal mobile content downloading in device-to-device communication underlaying cellular networks", IEEE Transactions on Wireless Communications, 13(7):3596-3608, 2014 
[13] W. Zhou, X. Sun, C. Ma, J. Yue, H. Yu, and H.-W. Luo, "An interference coordination mechanism based on resource allocation for network controlled Device-to-Device communication", IEEE/CIC International Conference on Communications, China, 2013

[14] Y. Xu, R. Yin, T. Han, and G. Yu, "Interference-aware channel allocation for device-to-device communication underlaying cellular networks", 1st IEEE International Conference on Communications in China (ICCC), 2012

[15] S. Wen, X. Zhu, Z. Lin, X. Zhang, and D. Yang, "Optimization of interference coordination schemes in device-to-device (D2D) communication", 7th International ICST Conference on Communications and Networking in China (CHINACOM), 2012

[16] T. Han, R. Yin, Y. Xu, and G. Yu, “Uplink channel reusing selection optimization for device-to-device communication underlaying cellular networks", IEEE 23rd International Symposium on Personal Indoor and Mobile Radio Communications (PIMRC), 2012

[17] B. Wang, L. Chen, X. Chen, X. Zhang, and D. Yang, "Resource allocation optimization for device-to-device communication underlaying cellular networks", IEEE 73rd Vehicular Technology Conference (VTC Spring), 2011.

[18] L. Song, D. Niyato, Z. Han, and E. Hossain, "Game-theoretic resource allocation methods for device-to-device communication", IEEE Wireless Communications, 21(3):136-144, 2014

[19] J. Gu, S. J. Bae, B.-G. Choi, and M. Y. Chung, "Dynamic power control mechanism for interference coordination of device-to-device communication in cellular networks", 3rd International Conference on Ubiquitous and Future Networks (ICUFN), pp. 71-75, IEEE, 2011

[20] S. J. Bae, D. H. Kim, B.-G. Choi, and M. Y. Chung, "Transmission power control for FlashLinQ device-to-device communication system", IEEE 77th Vehicular Technology Conference (VTC Spring), 2013

[21] S. Wen, X. Zhu, X. Zhang, and D. Yang, "QoS-aware mode selection and resource allocation scheme for device-to-device (D2D) communication in cellular networks", IEEE International Conference on Communications Workshops (ICC), 2013

[22] E. Zihan and K. W. Choi, "Random access protocol for collision avoidance in cellular device-to-device communication", IEEE International Conference on Communications (ICC), 2014 
[23] Z. Wang, E. K. Tameh, and A. R. Nix, "Statistical peer-to-peer channel models for outdoor urban environments at $2 \mathrm{GHz}$ and $5 \mathrm{GHz}$," in Proc. IEEE Veh. Technol. Conf., vol. 7, Los Angeles, USA, Sep. 2004, pp. 5101-5105.

[24] Further Advancements for E-UTRA. Physical Layer Aspects, 3rd Generation Partnership Project 3GPP TR 36.814 V1.5.0, Nov. 2009, Release 9.

[25] Evolved Universal Radio Access (E-UTRA); Physical layer procedures, 3rd Generation Partnership Project 3GPP TS 36.213 V9.3.0, Sep. 2010, Release 9. 
TIME HOPPING FOR DEVICE-TO-DEVICE COMMUNICATION IN LTE CELLULAR SYSTEM

\author{
by \\ Chen, T., Charbit, G. and Hakola, S. 2010 \\ Proceedings of IEEE Wireless Communications and Networking Conference 2010 \\ $\left(\mathrm{WCNC}^{\prime} 10\right)$
}

Reproduced with kind permission by IEEE 


\title{
Time Hopping for Device-to-device Communication in LTE Cellular System
}

\author{
Tao Chen, Gilles Charbit, Sami Hakola \\ Nokia Devices \\ Tao.t.chen@nokia.com
}

\begin{abstract}
In this paper, we analyze the challenges of the interference environment in a hybrid network that consists of the device-to-device (D2D) communication based ad-hoc connections and conventional cellular connections. Accordingly, time hoppin (TH) based radio resource allocation schemes aiming to improve the robustness of the hybrid network are proposed. The performance improvement even with the simple random $T$ scheme is considerable, which is validated via extensive simulations. In addition, the simulation results also give some hints on the optimization of the practical $\mathrm{TH}$ operation in a hybrid network.
\end{abstract}

Index Terms-Time Hopping, LTE, Device-to-device

I. INTRODUCTION

In the future, one big challenge for the mobile operator would be that the heavily growing infrastructure investmen fulfilling the demands of the exponential growth of mobile traffic is more and more unproportional to the slowly increasing profit. Thus, the high efficient cellular networks and value-added services are critical for the operators. Long Term Evolution (LTE) with the peak data rates of downlink (DL) $100 \mathrm{Mpbs}$ and uplink (UL) 50Mbps has been in 3GPP (e.g., [1] to fulfill the requirements of fast increasing mobile traffic which can significantly improve the spectrum efficiency and reduce transmission latency. Further, a hybrid network by integrating device-to-device (D2D) communication into LTE network is considered to further improve the network efficiency and enable some new value-added services, in which the $\mathrm{D} 2 \mathrm{D}$ devices (or mobiles or terminals or peers or machines) with LTE capability having a direct physical communication link can reuse LTE air interface for the local peer-to-pee traffic while sharing the cellular network resources with devices having a conventional cellular connection to the base station (a.k. eNB in LTE) for the exterior traffic. For example, the cellular network is operating in frequency example, the cellular network is operating in frequency
division duplex (FDD) mode in which D2D connections are division duplex (FDD) mode in which D2D connections are
utilizing time division duplex (TDD) mode either using cellular network UL and/or DL resources controlled by the base station(s). The general concept covering FDD or TDD cellular network where direct communication connection utilize eithe FDD or TDD duplex mode can be found from [2]. The purpose of adding direct communication into a cellular network is the possibility to reduce transmitter power consumption both in device and base station, increase cellular network capacity and establishing more services for the users, which is pointed out in [3]. Different with the relaying based hybrid network as mostly studied (e.g., in [4] and [5]), the D2D connection is mainly established according to the needs of the local peer-to-peer traffic but not for relaying the traffic to the base station or the user equipment (UE), which may lead to different gain mechanisms and resource allocation schemes. D2D integrated hybrid network is mainly targeting to offload the peer-to-peer traffic from the transportation network and reduce the
transmission delay via bypassing the access network. On the transmission delay via bypassing the access network. On the
other hand, D2D integrated hybrid network can be taken as the other hand, D2D integrated hybrid network can be taken as the
first step towards the mobile relaying system, in which the mobile can simultaneously support both D2D and cellular communication links.

Because of the challenges from the interference environment in this kind of hybrid network supporting both cellular and D2D communication, radio resource management (RRM) is getting more important to ensure the quality of service (QoS) and leverage the performance of cellular and D2D communication when sharing the same radio resources. In D2D communication when sharing the same radio resources. In
addition, even though the possible spatial reuse between D2D links is promising to improve the spectrum efficiency significantly, it may also lead to more variation on the system performance due to the co-channel interference from the reused D2D links, especially in the case of the imperfect spatial reuse.

In this study, the time hopping based radio resource allocation schemes rather than the typical power control solutions are proposed to solve the interference problems caused by the unideal spatial reused D2D devices in the hybrid network. D2D communication, operating in TDD mode by reusing LTE uplink transmitter in the device, is considered because of its minor implementation cost.

II. Challenges From InTERFERENCE ENVIRONMENT FOR D2D COMMUNICATION OPERATING IN LTE CELLULAR SYSTEM

A. Impact of near-far interferer on ADC range

The Analogue-to-Digital Converter (ADC) and Automatic Gain Control (AGC) typically work together to centre the dynamical range of the ADC around the stronger signal at the UE receiver, which could be the intended transmitting $(\mathrm{Tx})$ signal or a near-far Tx interfering signal. The resolution of an $\mathrm{ADC}$, denoted by $\mathrm{Q}$, is typically $\mathrm{Q}=[-\mathrm{V} ;+\mathrm{V}] / \mathrm{N}$, where $\mathrm{V}$ is the maximum magnitude and $\mathrm{N}$ the number of quantization levels. Considering an example with a 12-bit ADC converter (4096 levels), if the received power from the near-far interferer is 27 $\mathrm{dB}$ (500 times) higher than the wanted signal, the wanted signal magnitude would be represented by about $4 \mathrm{ADC}$ quantization levels $(\mathrm{v} / 500 \sim=4 * 2 \mathrm{~V} / 4096)$, which results in severe truncation. In practical ADC, the wanted signal may be removed entirely by ADC as some headroom is used as fading margin to avoid signal truncation due to positive fade. 
For TDD based D2D links transmitting and receiving in the same frequency band as LTE cellular users' transmission in the hybrid network, this kind of desired signal truncation suffering from the near-far interference is getting more severe than the pure cellular network. Usually, the LTE cellular users will transmit with the power level according to fractional power control (FPC) specified in [6] that compensates path loss between them and the base station but not limits the near-far interference to D2D receivers, which will cause the near-fa interference to D2D users in the case of frequency resource reusing. Especially, the cellular UEs in the cell edge typically transmitting at a high power are likely to create the stron interference to near-by D2D UEs in receive mode. Besides, the imperfect spatial reuse lacking of the real-time neighbor information may also lead to the strong co-channel interference among D2D users and even block the desired signals due to the snals due to the signal truncation in ADC/AGC. Otherwise, it is difficult to apply an efficient power control scheme as performed in the well planned and deployed cellular networks to ensure that al parallel transmitting signals arrive at the D2D receiver with the similar power level, because the deployment of the intended D2D receivers is not under the control of the operators, an also difficult to be predicted owing to the UE mobility and dynamics of the user behaviors.

B. Impact of Inter-carrier Interference (ICI)

Even in the case of frequency resource sharing between cellular users and D2D users, the inband emissions in UE transmissions due to imperfection of the radio frequency (RF) transceiver such as frequency offset and phase noise would destroy the orthogonality of the victim UE signal, consequently causing the inter-carrier interference (ICI), which has been investigated in [7]. The minimum requirements for inban emission have been also specified in [8] for LTE system to ensure that there is no significant performance degradation average across 12 subcarriers and as a function of the resource block (RB) offset (i.e., delta RB) from the edge of the allocated $\mathrm{UL}$ transmission bandwidth. It is measured as the [relative UE output power] of any non-allocated RB(s) and the [total UE output power] of all the allocated RB(s).

Figure 1 shows that depending on the allowed error vector magnitude (EVM) level of the selected modulation scheme, QPSK or 16QAM, the maximum allowed inband emissions can be around 18 or $21 \mathrm{~dB}$ for the small $\mathrm{RB}$ offset respectively, and leveling off around $25 \mathrm{~dB}$ for the larger $\mathrm{RB}$ offset.

Consequently, the near-far effect in D2D integrated LTE cellular network, where one interfering UE close to a D2D Rx UE may transmit at a much higher power than the D2D Tx UE, can aggravate the impact of ICI in the D2D UE receiver. Otherwise, it hints that the frequency orthogonality may not be Otherwise, it hints that the frequency orthogonality may not be
ideal due to the imperfection of RF transceiver in the device, ideal due to the imperfection of RF transceiver in the device,
and accordingly some typical solutions such as frequency and accordingly some typical solutions such as frequency
hopping used in pure cellular network may not be suitable for the resource allocation in the hybrid network.

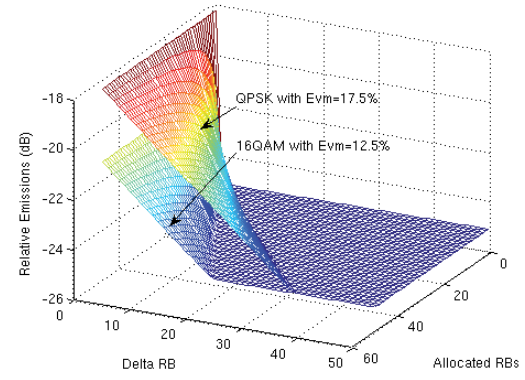

Figure 1. Illustration of inband emissions

III. Framework OF RADIO RESOURCE AlLOCATION

To reduce the near-far interference and improve the efficiency of the hybrid network, spatial/time/frequency orthogonality of LTE radio resource can be exploited for the resource allocation to device pairs/clusters and cellular user in the hybrid network as illustrated in Figure 2. For example, spatial reuse can be applied to distant pairs (e.g., P1 and P2/P3). Nearby pairs (e.g., P2 and P3) are allocated orthogonal resources in time/frequency domain. Coordination of orthogonal resource allocation for clusters located in differen cells (e.g. P2/P3 and P5) can be done via X2 interface between eNBs. The interference due to near-far problem is alleviated because of RB orthogonality and short distances of devices within the cluster. In practical operation, eNB will require a lot of measurement information from D2D users to apply the ideal radio resource orthogonality among D2D users. The tradeoff between the performance and the signaling overhead has to be taken into account in designing RRM of the hybrid network.



Figure 2. Illustration of resource allocation 
So, a decentralized scheduling for D2D users is considered for the resource allocation in the hybrid network. eNB allocates time/frequency resources to D2D users in a large time scale based on a middle or long term measurement with minor signaling overhead, whereas D2D pairs/clusters decide the usage of the resource adaptively in a small time scale according to the short term measurement. For instance, eNB may (semi-)persistently allocate some frequency resources to D2D users based on the measurement reports of D2D users, whereas D2D users with TDD channel reciprocity can make the link adaption based on its own instantaneous interference and channel conditions during the allocation period without any extra signaling to eNB.

\section{Principle of Time Hopping For D2D}

Time Hopping (TH) of D2D resources within the cell and across cells is carried out to randomize near-far interference from nearby transmitting D2D and cellular UEs. This kind of interference could be from co-channel interference and/or ICI. The base station (i.e., eNB in LTE) applies random sequence offsets at regular time intervals to semi-persistent scheduled resources, accordingly preventing one pair from excessively suffering eNB-unaware near-far interference by another pair for a longer duration. The random sequence offset used in time hopping of resources allocated to device pairs/clusters may be:

\section{- Based on device pair/cluster location.}

- In a short term for D2D devices within the same cell and spatially separated from other D2D devices in neighbor cells.

- In a long term via eNB co-ordination over LTE X2 interfaces with a latency of at least $20 \mathrm{~ms}$.

The principle of TH is to randomize the overall channe access collisions and minimize the occurrence of the collision between victim UEs and the strongest interfering UEs.

eNB can use Semi-Persistent Scheduler (SPS) as specified in [9] to schedule UL grant resources to a cluster head an slaves using with periodicity of the UL grant $\mathrm{sfx}(\mathrm{x}=10,20$, 120 in subframes). The clusters are formed by the eNB based on max D2D transmitting Power of UEs and geographical location estimation of devices (e.g. Timing Advance parameters and Direction of Arrival). UL grant resources parameters and Direction of Arrival). UL grant resources allocated to a cluster aim to minimize inter-cluster interference
by spatial orthogonality and/or time orthogonality (subfram by spatial orthogonality and/or time orthogonality (subframe separated), and if neither spatial nor time orthogonality is Note that frequency orthogonality is applied via separelf D2D communicati of adjacent RBs from other UE Tx, which has been demonstrated in figure 1 .

For example, the devices pairs P1, P2, P3, P4, and P5 in Figure 2 are allocated with TH sequence S1, S2, S3, S4, and S5 respectively. A simple random TH offset for device pair resources within a same geographical area (i.e. not spatially separated) is assumed. Let's denote the spatial group including device pairs $\{\mathrm{P} 1$ and $\mathrm{P} 4\}$ by $\mathrm{G} 1$, and $\{\mathrm{P} 2, \mathrm{P} 3\}$ by G2.

The time hopping procedure can be expressed as two steps:

Step 1: Divide the radio resources as several orthogona groups in the time domain, in which each group has a predefined periodical transmission timeslots as below:

$\mathrm{t}_{\mathrm{j}, 1}=\mathrm{NG}^{*}\{1,2,3, . ., \mathrm{NP}\}=\mathrm{NG}, 2 * \mathrm{NG}, 3 * \mathrm{NG}, \ldots ., \mathrm{NG} * \mathrm{NP}$

$\mathrm{t}_{\mathrm{j}, 2}=\mathrm{t}_{\mathrm{j}, 1}-1=\mathrm{NG}-1,2 * \mathrm{NG}-1, \ldots, \mathrm{NG}^{*} \mathrm{NP}-1$

$\mathrm{t}_{\mathrm{j}, \mathrm{i}}=\mathrm{t}_{\mathrm{j}, \mathrm{i}-1}-1=\mathrm{NG}-\mathrm{i}+1,2 * \mathrm{NG}-\mathrm{i}+1, \ldots, \mathrm{NG} * \mathrm{NP}-\mathrm{i}+1$

$\mathrm{t}_{\mathrm{j}, \mathrm{NG}}=\mathrm{t}_{\mathrm{j}, \mathrm{NG}-1}-1=1, \mathrm{NG}+1, \ldots, \mathrm{NG}^{*}(\mathrm{NP}-1)+1$;

Where NG denotes the number of TH resource groups, NP for the number of $\mathrm{TH}$ period, and $\mathrm{j}$ for the $\mathrm{TH}$ timeslot index expressed as a multiple on NP timeslots.

Step 2: D2D pairs are allocated to different resource groups in a period of NP via eNB SPS scheduler or the (random) selection by the $\mathrm{D} 2 \mathrm{D}$ pairs themselves.

For example, $\mathrm{NG}=3$ and $\mathrm{NP}=3$ may give timeslot index sequences $\mathrm{t}_{1,1}=\{3,6,9\}, \mathrm{t}_{1,2}=\{2,5,8\}, \mathrm{t}_{1,3}=\{1,4,7\}$, which in turn allocates timeslots $\{\mathrm{s} 3, \mathrm{~s} 6, \mathrm{~s} 9\},\{\mathrm{s} 2, \mathrm{~s} 5, \mathrm{~s} 8\}$, and $\{\mathrm{s} 1, \mathrm{~s} 4$ s7 \} to devices P2, P3, and P5 in first term of sequence S2, S3, and S5 respectively. Further, as shown in the example, the

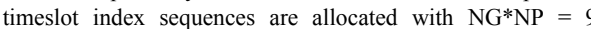
timeslot periodicity. Hence, we assign $\mathrm{t}_{\mathrm{j}, 1}, \mathrm{t}_{\mathrm{j}, 2}$, and $\mathrm{t}_{\mathrm{j}, 3}$ to device pairs $\mathrm{P} 1, \mathrm{P} 2, \mathrm{P} 3, \mathrm{P} 4$, and $\mathrm{P} 5$ with a random TH offset to define

TH sequences S1, S2, S3, S4, and S5 respectively as follows

$\mathrm{S} 1=\left\{\mathrm{t}_{1,2}, \mathrm{t}_{2,3}, \ldots\right\}=\{\mathrm{s} 2, \mathrm{~s} 5, \mathrm{~s} 8, \mathrm{~s} 10, \mathrm{~s} 13, \mathrm{~s} 16, \ldots\}$

$\mathrm{S} 4=\left\{\mathrm{t}_{1,1}, \mathrm{t}_{2,2}, \ldots\right\}=\{\mathrm{s} 3, \mathrm{~s} 6, \mathrm{~s} 9, \mathrm{~s} 11, \mathrm{~s} 14, \mathrm{~s} 17, \ldots\}$

$\mathrm{S} 2=\left\{\mathrm{t}_{1,3}, \mathrm{t}_{2,1}, \ldots\right\}=\{\mathrm{s} 1, \mathrm{~s} 4, \mathrm{~s} 7, \mathrm{~s} 12, \mathrm{~s} 15, \mathrm{~s} 18, \ldots\}$

$\mathrm{S} 3=\left\{\mathrm{t}_{1,1}, \mathrm{t}_{2,3} \ldots\right\}=\{\mathrm{s} 3, \mathrm{~s} 6, \mathrm{~s} 9, \mathrm{~s} 10, \mathrm{~s} 13, \mathrm{~s} 16, \ldots\}$

$\mathrm{S} 5=\left\{\mathrm{t}_{1,2}, \mathrm{t}_{2,2}, \ldots\right\}=\{\mathrm{s} 2, \mathrm{~s} 5, \mathrm{~s} 8, \mathrm{~s} 11, \mathrm{~s} 14, \mathrm{~s} 17, \ldots\}$

Note that the same TH timeslot index sequence, $\mathrm{t}_{\mathrm{j}, \mathrm{i}}$, may not be used by more than one device pair within the same spatial group to prevent intra near-far interference. Compare this set of sequences using random $\mathrm{TH}$ offset to the following sequence set without such $\mathrm{TH}$.

$\mathrm{S}^{\prime}=\left\{\mathrm{t}_{1,1}, \mathrm{t}_{2,1}, \ldots\right\}=\{\mathrm{s} 3, \mathrm{~s} 6, \mathrm{~s} 9, \mathrm{~s} 12, \mathrm{~s} 15, \mathrm{~s} 18, \ldots\}$

$\mathrm{S}^{\prime}{ }^{\prime}=\left\{\mathrm{t}_{1,2}, \mathrm{t}_{2,2}, \ldots\right\}=\{\mathrm{s} 2, \mathrm{~s} 5, \mathrm{~s} 8, \mathrm{~s} 11, \mathrm{~s} 14, \mathrm{~s} 17, \ldots\}$

$\mathrm{S}^{\prime}{ }^{\prime}=\left\{\mathrm{t}_{1,2}, \mathrm{t}_{2,2}, \ldots\right\}=\{\mathrm{s} 2, \mathrm{~s} 5, \mathrm{~s} 8, \mathrm{~s} 11, \mathrm{~s} 14, \mathrm{~s} 17, \ldots\}$

$\mathrm{S} 3^{\prime}=\left\{\mathrm{t}_{1,1}, \mathrm{t}_{2,1}, \ldots\right\}=\{\mathrm{s} 3, \mathrm{~s} 6, \mathrm{~s} 9, \mathrm{~s} 12, \mathrm{~s} 15, \mathrm{~s} 18, \ldots\}$

$\mathrm{S}^{\prime}{ }^{\prime}=\left\{\mathrm{t}_{1,3}, \mathrm{t}_{2,3}, \ldots\right\}=\{\mathrm{s} 1, \mathrm{~s} 4, \mathrm{~s} 7, \mathrm{~s} 10, \mathrm{~s} 13, \mathrm{~s} 16, \ldots\}$

S1', S2', S3', S4', and S5' can readily be scheduled by SPS. Note that the SPS allocates UL grants every sfx subframes as specified in [10]. Hence, device pairs P1, P2, P3, P4, and P5 are allocated UL grants by SPS every $\mathrm{sfx}=9$ subframes. Further, consider the case where transmitting devices in device pairs $\mathrm{P} 4$ in spatial group G1 may cause strong interference to a receiving device in device pairs $\mathrm{P} 2$ in spatial group G2. Thi could be the case there is no effective spatial orthogonality between $\mathrm{P} 4$ and $\mathrm{P} 2$, perhaps due to devices in $\mathrm{P} 4$ being in an elevated position or having a direct line of sight (LOS) to devices in P2. In the example, without $\mathrm{TH}$, device pair $\mathrm{P} 4$ are allocated UL grants by SPS on the same time slots as device 
pair P2 and will cause interference on all the timeslots.

The near-far interference with and without $\mathrm{TH}$ for thi example is illustrated in Figure 3. So, with TH, only a few time slots allocated to device pair P2 will suffer such interference from device pair P4.

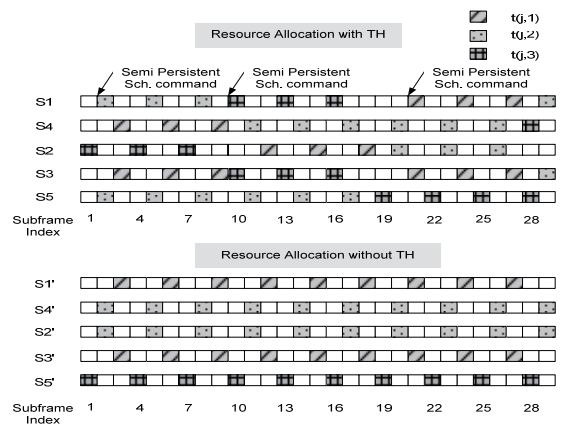

Figure 3. Illustration of resource allocation w/o TH

Comparing to the TH with eNB SPS scheduler, the event triggered $\mathrm{TH}$ and random $\mathrm{TH}$ by $\mathrm{D} 2 \mathrm{D}$ pairs can be performed with less signaling overhead.

V. SimUlation Scenarios And Results Analysis

To simplify the simulation without loss of generality, the performance of the random TH without ICI is investigated. A quasi-static simulator is established for the evaluation of the performance with multiple drops. The cell layout is shown in figure 4. D2D Tx UEs are evenly distributed in each cell, an Rx UEs are located around the Tx UEs with a pre-defined maximum distance (i.e., D2D circle radius). LTE FPC is used as UL power control scheme for D2D users, which can prevent the eNB receivers from the excessive D2D interference. Note that only one frequency resource is modeled.



Figure 4. Cell layout with several D2D circles per cell, each D2D circte (cyan circle) including one Tx UE (blue circle) and one Rx UE (green circle)
Main parameters used in the system simulation are summarized in Table I.

LIST OF SIMULATION PARAMETERS
\begin{tabular}{|l|l|}
\multicolumn{1}{|c|}{ Parameter } & \multicolumn{1}{c|}{ Value } \\
\hline Inter site distance & $0.5 \mathrm{~km}$ \\
\hline Cell configuration & 19 cells \\
\hline Cellular link pathloss model & PL $=128.1+37.6 * \log 10\left(\mathrm{R} \_\mathrm{km}\right)$ \\
\hline D2D link pathloss model & $\mathrm{PL}=127+30^{*} \log 10\left(\mathrm{R} \_\mathrm{km}\right)$ \\
\hline Cellular UEs per cell & $1 \mathrm{UE}$ for case A; 0 UE for case B and C. \\
\hline D2D pairs per Cell & $3,6, \ldots, 18$ pairs \\
\hline Max D2D circle radius & $20,40, \ldots, 200 \mathrm{~m}$ \\
\hline TH groups $(\mathrm{NG})$ & $3,6, \ldots, 18$ \\
\hline TH period $(\mathrm{NP})$ & $1,2,3,4,8,16,32$ \\
\hline Time length for non-TH & 32 \\
\hline Cellular link PC setting & FPC $\left(\alpha=0.6, P_{0}=-56 \mathrm{dBm} / \mathrm{RB}\right)$ \\
\hline D2D link PC setting & FPC $\left(\alpha=0.6, P_{0}=-56 \mathrm{dBm} / \mathrm{RB}\right) ;$ \\
\hline
\end{tabular}

\section{A. Effect of near-far interferers}

Firstly, it is worth of checking the impact of near-far interferes on the performance. As shown in figure 5, with increasing max D2D radius, the weakness on the wanted signal due to the increased pathloss and relatively increased near-far interferers would make the performance worse. When the number of D2D pairs is quite small on the allocated frequency resource, the performance difference between reusing and not reusing the same frequency resource with cellular users are quite considerable. In this case, the interference from intra/inter cell cellular users are dominating. On the other hand, with increasing of the reusing D2D pairs, the interference from D2D pairs is prevalent, thus obscuring the pe
the cases w/o reusing with cellular users.

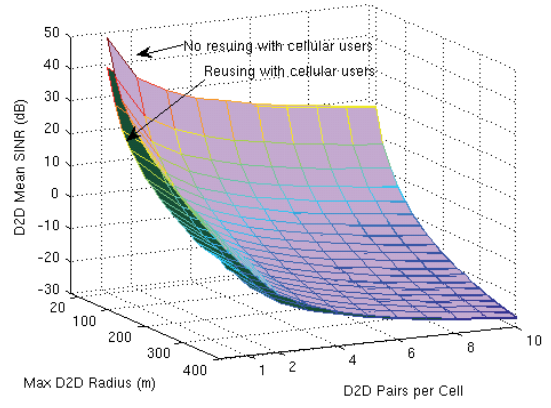

Figure 5. Impact on the performance from near-far interferes

So the investigation of $\mathrm{TH}$ in the remaining sections would mainly focus on the scenarios, in which multiple D2D pairs are reusing the same frequency resource by ignoring the effect of the cellular users. 
B. Effect of TH on the performance in general

In this section, the performance with $\mathrm{NP}=3$ for $\mathrm{TH}$ is evaluated in the scenarios with different max D2D radius and D2D traffic load in order to study whether the gain from TH is general or not.

As shown in figure 6, the performance regarding to the mean SINR received by D2D UEs with $\mathrm{TH}$ is quite similar to that without $\mathrm{TH}$.

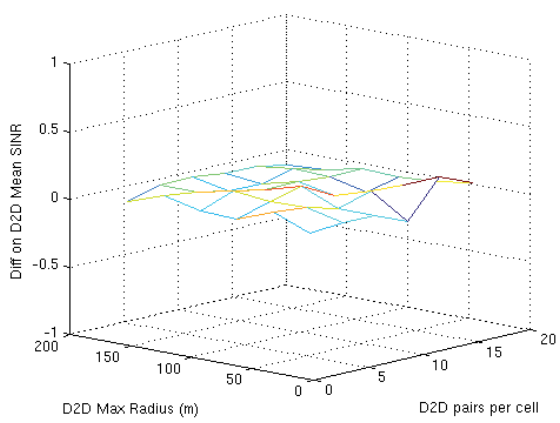

Figure 6. Difference on D2D mean SINR values.

However, the Std SINR values with TH are far below the reference cases without $\mathrm{TH}$ as presented in figure 7 . With increasing of the resource group number, the difference on the reduction of Std SINR is not significantly any more.

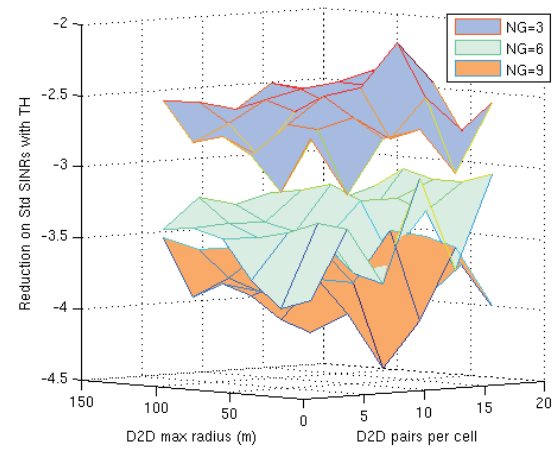

Figure 7. Impact on the performance from near-far interferes with $3 \mathrm{D} 2 \mathrm{D}$ pairs per cell.

Furthermore, the SINR distribution in figure 8 can exemplify the improvement on the performance by $\mathrm{TH}$ for the worst D2D UEs that are heavily interfered by nearby D2D pairs. The reason for the gain from $\mathrm{TH}$ is such that, it can reduce the D2D interference efficiently by exploiting the characteristics of ideal orthogonality in the time domain to randomize the interference from neighbor transmitting UEs. Besides, it is expected to obtain more gain via fully orthogonal $\mathrm{TH}$ sequences allocated to mutual interfering D2D pairs.

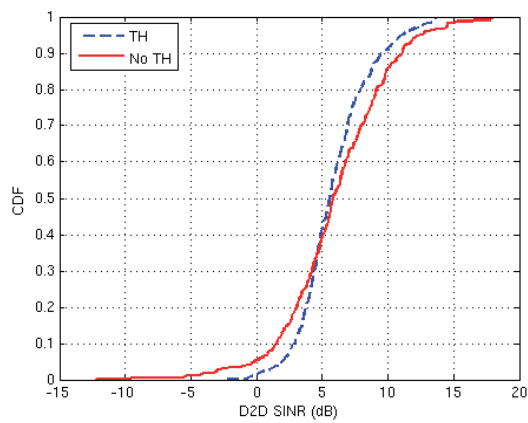

Figure 8. D2D SINR distribution w/o T

In summary, the gain from TH is quite general and holds fo most scenarios regardless of the D2D distance and traffic load. However, the concrete gain may depend on the parameters settings, which is studied in the following section.

C. Effect of TH parameters on the performance

In this section, two key parameters of $\mathrm{TH}$, the time resource group number and $\mathrm{TH}$ period, are investigated to provide guideline for the operation of $\mathrm{TH}$

As shown in figure 9, more resource groups in the time domain can reduce the co-channel interference significantly, thus achieving the higher SINR via the ideal orthogonality in the time domain.



Figure 9. Effect of the grouping number and TH period on mean SINR with 3 D2D pairs per cell.

On the other hand, it makes the performance more sensitive to the co-channel interference in this high SINR region wher 
any access collision would deviate SINR far away from the mean value, which is more obvious for the reference cases in figure 10 . In addition, 3 D2D pairs per cell are having the higher Std SINRs than the case with 18 pairs per cell under the same configuration of $\mathrm{NG}$, which means that the large grouping number can improve the mean SINR but deviate SINRs heavily, especially in the case that there are only a few D2D pairs.

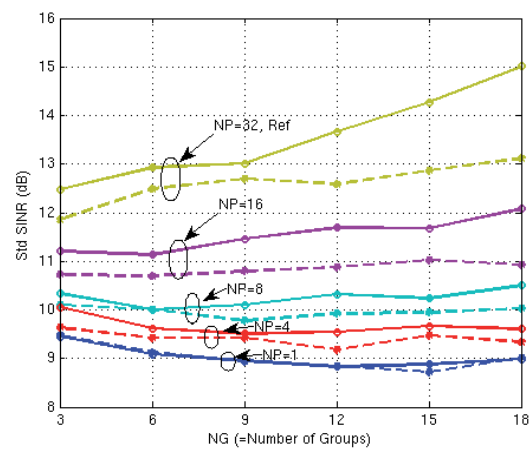

Figure 10. Effect of the grouping number and TH period on Std SINR with different D2D load, solid line for 3 pairs/cell and dashed line for 8 pairs/cell.

However, with increasing the number of resource groups in the time domain, TH can enlarge the gain in terms of the reduction on Std SINR. It is because TH can efficiently randomize some occasionally happened collisions comparing to the reference cases. According to the results, it seems that grouping into 3 groups can spare enough room in the time domain for TH to obtain the most gains. Otherwise, it can be noted that more groups may result in a larger transmission delay since the D2D user has to wait a longer time for the next transmission.

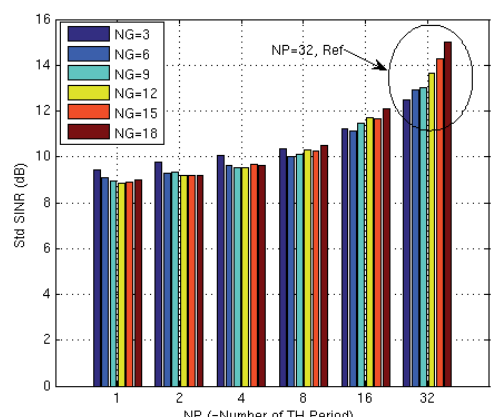

Figure 11. Effect of the grouping number and TH period on Std SINR.
Comparing to the effect of the grouping number, the $\mathrm{TH}$ period settings have more impact on the Std SINR as shown in figure 11. It can be noted that up to $6 \mathrm{~dB}$ reduction on Std SINR can be obtained at $\mathrm{NP}=1$ while the mean SINR is kept at the same level as the reference cases. Basically, $\mathrm{NP}=4$ seems good enough to get most gains with a minor cost on the signaling.

In general, the results provide some hints for the optima configuration, i.e., increasing the grouping number can improve mean SINRs, whereas reducing TH period can improve Std SINRs. Thus, the adjustment of the parameters depending on Quality of Service (QoS), delay requirements and D2D load may be an efficient way to optimize the performance.

\section{CONCLUSION}

Time hopping of D2D resources within cell and across cells is carried out to randomize near-far interference from nearby transmitting D2D UEs and also nearby transmitting cellular UEs, which can reduce problematic near-far interference in D2D communications efficiently. The gain from TH hopping is general rather than specific to some cases. The optimization of the TH parameter settings can further improve the overal performance depending on the service requirements. Further, it is easy to operate TH because of the low signaling overhead and a set of limited choices for the parameters optimization.

\section{REFERENCES}

[1] 3GPP TS 36.300, v9.1.0, "E-UTRA and E-UTRAN; Overall description", September 2009 .

[2] S. McLaughlin and H. Haas, "Cellular communications system", Patent Application WO060182, 2005.

[3] R1-082813, "M2M Communications", Alcatel-Lucent, 3GPP RAN1\#54, 18-22 August 2008 .

[4] H.-Y. Hsieh and R. Sivakumar, "On Using the Ad-hoc Network Model (

[5] Lap Kong Law, Srikanth V. Krishnamurthy, and Michalis Faloutsos, "Capacity of Hybrid Cellular-Ad hoc Data Networks", INFOCOM 2008

[6] TS 36.213, "E-UTRA Physical layer procedures" v8.4.0, September 2008 .

[7] Basuki E. Priyanto, Troels B. Sørensen, Ole K. Jensen, "In-Band Interference Effects on UTRA LTE Uplink Resource Block Allocation", VTC spring 2008.

8] 3GPP TS 36.101, v8.1.0, "User Equipment (UE) radio transmission and reception", March 2008.

[9] 3GPP TS 36.321, v8.3.0, "EUTRAN Medium Access Control"

[10] 3GPP TS 36.331, v8.3.0, "EUTRAN Radio Resource Control" 
VIII

\title{
DEVICE-TO-DEVICE COMMUNICATION UNDERLAYING CEL- LULAR NETWORKS-CONNECTION ESTABLISHMENT AND IN- TERFERENCE AVOIDANCE
}

\author{
by \\ Xu, S., Wang, H., Chen, T., Peng, T., Kwak, K., S. 2012 \\ Journal of KSII Transactions on Internet and Information Systems, \\ vol 6, Page 203-228
}

Reproduced with kind permission by KSII 


\title{
Device-to-Device Communication Underlaying Cellular Networks: Connection Establishment and Interference Avoidance
}

\author{
Shaoyi $\mathrm{Xu}^{1,2}$, Haiming $\mathrm{Wang}^{3}$, Tao Chen ${ }^{3}$, Tao Peng ${ }^{4}$ and Kyung Sup Kwak \\ ${ }^{1}$ School of Electronic and Information Engineering, Beijing Jiaotong University \\ Beijing, 100044, P. R. China \\ ${ }^{2}$ State Key Laboratory of Integrated Services Networks, Xidian University \\ Xian, 710071, P. R. Chin \\ [e-mail: shyxu@ bjtu.edu.cn] \\ ${ }^{3}$ Renesas Mobile \\ [e-mail: \{haiming.wang, tao.chen\}@renesasmobile.com] \\ ${ }^{4}$ Beijing University of Posts and Telecommunications \\ Beijing, 100876, P. R. China \\ [e-mail: pengtao@bupt.edu.cn] \\ ${ }^{5}$ UWB Research Center, Inha University \\ Incheon, 402-751, Korea \\ Incheon, 402-751, Korea \\ *Ce-mail: kskwak@inha.ac.kr]
}

Received September 02,2011; revised November 16, 2011; January 4, 2012

Published January 31,2012

\begin{abstract}
It is expected that device-to-device (D2D) communication is allowed to underlay future cellular networks such as IMT-Advanced for spectrum efficiency. This article studies the mechanisms of D2D communication and interference avoidance when the D2D subsystem reuses uplink resources and downlink spectrums with a cellular system, respectively. We firstly propose an effective scheme to establish and maintain D2D communication. Moreover, a novel method to deal with the resource allocation and interference avoidance issues by utilizing the network peculiarity of a hybrid network to share the uplink resource is proposed. Most research focuses on reusing the uplink spectrums, but how to share the downlink frequency bands is seldom addressed. To share the downlink spectrums and avoid the interference to the primary cellular devices, a labeled time slots based mechanism is proposed. Implementation details are described in a real cellular system and simulation results prove that satisfying performance can be achieved by using the proposed mechanisms.
\end{abstract}

Keywords: Device-to-device communication, hybrid system, near-far interference, long term evolution, labeled time slots

A preliminary version of this paper appeared in IEEE VTC Spring 2010, May 16-19, Taiwan, China. This version includes a concrete analysis and supporting implementation of connection establishment, communication maintenance, a power saving scheme and some extensive simulations. This work was supported in part by the Important National Science \& Technology Specific Projects of China with Grant 2012ZX03003013-003, National Natural Science Foundation of China with Grant 61101138 and by the MKE (Ministry of Knowledge Economy), Korea, under the ITRC (Information Technology Research Center) support program supervised by the IITA (Institute of Information Technology Assessment). DOI: 10.3837/tiis.2012.01.012 


\section{Introduction}

$\mathbf{R}$ ecently, major efforts have been spent on the development of Third Generation Partnership Project (3GPP) Long Term Evolution (LTE) for the higher data rate and system capacity. Showing substantial performance improvements in the throughput and spectrum efficiency, LTE is foreseen to be a solid ground for the future IMT-Advanced (IMT-A) technology [1]. IMT-A will offer a high bandwidth up to $100 \mathrm{MHz}$ for higher data rates, global operation and economy of scales supporting a wider range of services. Many candidate radio interface technologies have been submitted to the International Telecommunications Union (ITU) to prepare new technology components for LTE to meet IMT-A requirements. Among which, device-to-device (D2D) communication has received increasing attentions as a promising component to improve spectrum efficiency [1][2][3].

Unlike the infrastructure based cellular network, D2D users (user equipments or mobile terminals) do not communicate via the central coordinator (base station, NodeB or evolved NodeB) but operate as an underlay and communicate directly with each other or more hops. Excluding the unnecessary core network involvement, D2D communication is an appealing concept which can provide several advantages such as low cost, plug-and-play convenience, and flexibility. Its usage of bandwidth and battery power is more efficient and interference can be reduced if two near terminals in different cells communicate directly [4][5][6][7][8]. Furthermore, increased network efficiency supports more services or improves current services and applications. The services which can possibly benefit from direct communication include information sharing, mobile multiplayer gaming, mobile advertising, streaming services, social or community services with D2D and extending D2D concepts to a mobile relay [9].

Indeed, such D2D communication is likely to become integral to the future beyond $3 \mathrm{G}$ world to form a hybrid network [8][10]. Fig.1 illustrates the basic and extended concept of D2D communication and hybrid networks. In Fig. 1, a user equipment (UE) can communicate with another UE directly or by the aid of a base station. Moreover, multiple D2D subsystems can share resources in a cell.

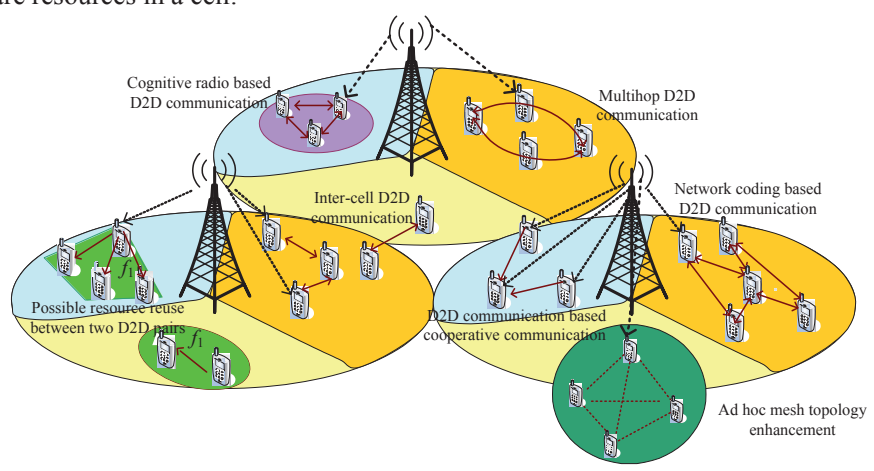

Fig.1. Illustration of D2D communication as an underlay to a cellular system. 
Actually, the trait of D2D communication is not new in the cellular system and it has been identified in some specifications such as Private Radio Communication System (PRCS), Japanese Handy Phone System (HPS), Digital Enhanced Cordless Telecommunications (DECT), etc. Many companies devote actively much in this research. Qualcomm, for example, is heading and has been very active recently. A prototype of "FlashLinQ" modem has been developed since 2006 to enable direct D2D communication over a licensed spectrum. The prototype effort covers the protocol stack from the host software interface to the physical layer algorithm. "FlashLinQ" is even a new trademark registered in 2008 by Qualcomm [11].

The key motivation for utilizing D2D communication underlaying a cellular network is to keep local communication local. D2D communication shares the same resources with the cellular system whereas under the control of the evolved NodeB (eNB). In such a case, an eNB can still control the resource and power assigned for D2D transmission to limit the interference to the primary cellular networks. Although the concept of D2D communication as an underlay to a cellular network is not new in a cellular system, it is new in LTE systems. As a result, some new challenges are incurred. The first key issue to be resolved is how to facilitate D2D communication in the licensed frequency band. Different from a centralized system where an eNB fully controls the data transmission which should be sent on the pre-allocated resources and UEs must synchronize with the eNB, devices would compete with each other for the resource in a distributed way in the D2D subsystem. Secondly, interference management in a hybrid network is a critical issue in that the interference from D2D links can be expected to reduce the cellular capacity and efficiency and vice versa. In this paper, focusing on frequency division duplex (FDD) LTE networks, we concentrate on the D2D communication establishment and maintenance, and near-far interference avoidance.

There has been some research on D2D communication underlaying a cellular network. [1] proposed a mechanism for $\mathrm{D} 2 \mathrm{D}$ communication session setup and management along with the interference coordination between two subsystems in the LTE system architecture evolution. To manage the interference between D2D and cellular networks, in [12], by assuming that an eNB completely controls over the D2D links, a power control scheme is suggested to maximize the system sum throughput. [3] also applied a power control method to mitigate the interference by using the channel statistics instead of instantaneous channel state information of the links between the cellular UEs and the D2D users. [7] formulated the power control problem as a mixed integer nonlinear programming and further proposed a greedy heuristic algorithm to realize this method. By employing a game theory based power control scheme, an interference suppression mechanism is studied in [13]. A time hopping based mechanism rather than a typical power control scheme is presented in [6]. The author suggested that interference among multiple D2D pairs is randomized by allocating different time hopping sequence offsets among them.

We make three main contributions in this work. We firstly propose a feasible and effective D2D communication mechanism named Synchronized Slotted Aloha with RTS/CTS (SSARC) to implement an autonomous handshake, competition and data transmission among D2D users. By synchronizing with the primary cellular system and following the same time structure with it, D2D users compete for transmission priority based on the Aloha with Request to Send/Clear to Send (RTS/CTS) mechanism and then the D2D communication is maintained to reuse the cellular uplink (UL) resources. To best of our knowledge, no such similar work was proposed before.

Furthermore, when UL spectrums are reused, the victim device is commonly considered to be the eNB and several algorithms are studied to coordinate this interference by reducing transmission power of D2D users [1][7][12]. However, with respect to the amount of cellular 
users and the short distance between two D2D UEs, it is D2D users that have higher possibility to be interfered. In our work, we address the near-far interference to the D2D devices and propose a proactive interference avoidance scheme by utilizing the helpful information from the eNB.

Due to heavier traffic and fast resource scheduling in LTE downlink (DL) transmission, most work emphasized that D2D devices make use of the cellular UL spectrum [9][13][14][15][16][17]. Different from the previous research, we propose a novel interference avoidance scheme to reuse DL resources by using labeled time slots. Extensive simulations prove the better efficacy of our method over the traditional power control scheme.

The paper will be organized as follows. In Section 2, we present the procedure of D2D transmission establishment and maintenance aiming to realize efficient resource utilization in the hybrid network. Since D2D devices work autonomously and fully share frequency resources with cellular users, sometimes there is near-far interference between cellular users and D2D pairs. In Section 3, we propose a novel scheme to realize UL resources sharing meanwhile avoiding near-far interference to D2D transmission in a hybrid network. Furthermore, Section 4 describes the mechanism and implementation details to reuse DL resources by applying labeled time slots. Finally, performance evaluation and discussions are presented in Section 5 and conclusions are given in Section 6.

\section{Establishment and Maintenance of D2D Communication}

\subsection{Prior art overview}

The establishment and maintenance of D2D communication is a preliminary problem to enable D2D sessions. Since D2D terminals will not be under the tight control of an eNB and D2D subsystems share the resource with their anchored cellular systems, consequently, several questions are thus raised. Firstly, D2D communication may exert serious interference to the cellular system or it may be interfered by the cellular users. Thus, how to coordinate the interference between the two subsystems? Furthermore, there maybe exist multiple D2D pairs which initiate their communication simultaneously such that inter-pair interference happens. Then the competition problem among them should be solved efficiently to avoid such harmful interference. Our third concern is how to synchronize those D2D partners to avoid disordering the transmission. That is a question on how to inform the D2D transmitter and receiver when and on which resource to start the transmission. To answer these questions, let us first investigate several currently proposed methods. In this article, a D2D transmitter and receiver are abbreviated as 'Tx_D_UE' and 'Rx_D_UE' separately.

Prior Art 1: eNB arbitrarily controlling D2D communication

One intuitive solution is to allow an eNB to control the D2D communication arbitrarily. Fig. 2 depicts the procedure and signaling of this mechanism. Obviously, D2D transmission is completely controlled by the eNB.

The advantage of this approach is that the eNB is able to easily handle the procedure for all the D2D transmission, and it is unnecessary to worry about the mess caused by any uncertainties. Moreover, resource allocation can be performed completely by the eNB such that less interference exists in such networks. Inevitably, this method has several disadvantages. Since the eNB takes charge of the whole D2D communication procedure, all the D2D related signaling consumes the radio resources of the cellular network and may reduce the throughput and capacity of a cellular network. Another, if a large number of D2D 
UEs transmit the data at the same time, the amount of D2D signaling would be huge. Furthermore, when the D2D partners are at the edge of different cells, it will become worse. Under such a situation, extra information (such as the pathloss between D2D users) would be required by an eNB to make radio resource management (RRM) decision for D2D data transmission. Otherwise, signaling needs to be exchanged over the X2 interface between neighboring eNBs for the resource coordination which will result in a long delay.

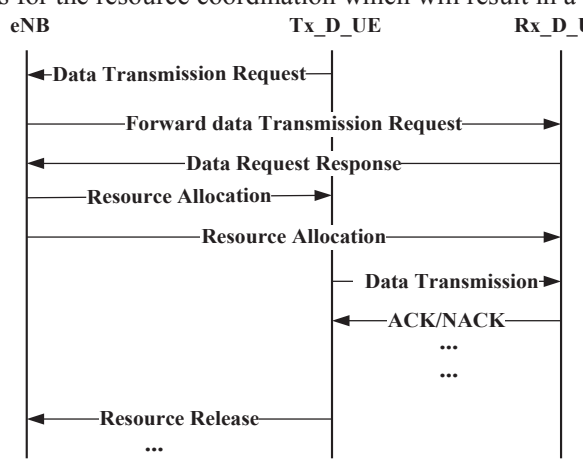

Fig. 2. An example of D2D transmission under arbitrary control of an eNB.

\section{Prior Art 2: Resource contention based D2D communication}

Another straightforward solution is a purely autonomous solution, which uses the similar schemes to those for Ad-hoc networks, Carrier Sense Multiple Access/Collision Avoid (CSMA/CA) with or without RTS/CTS, which are adopted in IEEE 802.11.

Notwithstanding this contention based framework can effectively resolve the data collision between D2D pairs in the hybrid network, it is challenged by threefold shortcomings. Firstly, the signaling such as RTS, CTS and ACKnowledgement (ACK), Negative ACKnowledgement (NACK) uses the same channel resources as the data transmission which results in the fact that data and signaling can not be transmitted simultaneously. Secondly, Distributed Interframe Space (DIFS) and Short Interframe Space (SIFS) are necessary to reduce the possibility of collisions, however, in the sensing duration data transmission is impossible thus leading to low resource utilization efficiency. Especially in the scenario where there are lots of short data to transmit, the overhead will be quite high. Finally, such a contention based mechanism can not avoid the interference from the cellular communication efficiently when sharing the licensed cellular system frequency band.

As a summary, a smart D2D RRM is necessary to control the interference and improve the performance. It requires that the solution should be able to take advantage of all the available information, including the signaling in the cellular network, to facilitate D2D RRM functions, such as frequency resource allocation, power control, interference avoidance, and so forth.

To this end, we propose an autonomous D2D communication mechanism named SSARC, which is assisted by the infrastructure system. The key point of this novel mechanism is a synchronized slotted Aloha with RTS/CTS, which achieves the handshake, competition and data transmission between D2D users effectively and efficiently. 


\subsection{Autonomous D2D communication assisted by the infrastructure system}

In the initial stage, D2D UEs should firstly register to one cell to obtain some essential information from an eNB such as whether it is D2D capable or not, Cell-ID of the anchored cell, fractional power control parameters used by this cell and the allocated Cell Radio Network Temporary Identifier (C-RNTI). It also should be noted that before the D2D communication is established, it is necessary for two neighboring eNBs to exchange their D2D devices information to smooth the communication when two or multiple terminals are located in different cells.

To implement the successful handshake procedure, it is necessary to define several channels for D2D communication which are illustrated in Fig. 3.

Common Control Channel (CCCH): $\mathrm{CCCH}$ is used by the D2D pair to claim a set of dedicated resource for D2D communication in a contentious way using the RTS/CTS based mechanism. At the beginning of D2D transmission, the eNB broadcasts the reserved resource including time and frequency of $\mathrm{CCCH}$ in the broadcasting channel $(\mathrm{BCH})$ such that all cellular UEs and D2D pairs know the information of D2D CCCH. To increase the spectrum efficiency, different D2D pairs share the same CCCH based on the Time Division Multiple Access (TDMA) mode.

Data Control Channel (DCCH): DCCH is used to carry data associated control information such as ACK/NACK and data control information (DataCtrl). DCCH resource corresponding to a certain $\mathrm{CCCH}$ would be reserved to ensure no collision with other D2D users and cellular communication. In our approach, DataCtrl signaling and ACK/NACK feedback are sent on the even TTI and odd TTI separately to improve the spectrum usage and avoid the possible collisions among multiple D2D transmission. To realize this trait, 1-bit information is needed to show the even or odd TTI by the eNB companying with the DCCH reserved resource information on the $\mathrm{BCH}$.

Data Traffic Channel (DTCH): DTCH is used to carry the D2D data traffic by reusing the UL spectrum with LTE users. It is actually not a specific channel for D2D users but a part of Physical Uplink Shared Channel (PUSCH) in the LTE system. Channel qualification information (CQI) could be carried on CTS signaling to provide channel condition information for the Tx_D_UE. Consequently, adaptive modulation and coding can be applied for $\mathrm{D} 2 \mathrm{D}$ communication based on the CQI

To reuse the spectrum between PUSCH and DTCH, the eNB sends the C-RNTIs of all cellular users to D2D UEs to decode the uplink transmission resource allocations (UL MAP) and performs pre-detections of interference. The UL MAP information is sent on the Physical Downlink Control Channel (PDCCH) which is encrypted by the C-RNTI. After obtaining the UL MAP, D2D UEs monitor the corresponding UL transmission channel to detect the interference. This detection may continue some periods until a list of average interference can be built. From this list, D2D UEs can find which cellular UE may exert slight interference on it which implies that this D2D UE is far away from this cellular user and can share the same resource with it. Here, an optimal threshold for slight interference depends on some factors, such as the number of D2D UEs and cellular UEs in a cell, the distance between a D2D UE and a cellular UE, the transmission power of the cellular UE, etc. Commonly, the optimal threshold can be set up by the operator according to an experiential value. In the following D2D data transmission, D2D UEs will share the same resource with this cellular UE. Decoding the C-RNTIs of all users is unavailable for the cellular UEs in the current LTE systems, whereas it is expected to be a common ability for the users in the IMT-A network. To 
comprehend the detailed procedure and signaling of this solution, please refer to our previous work [13].

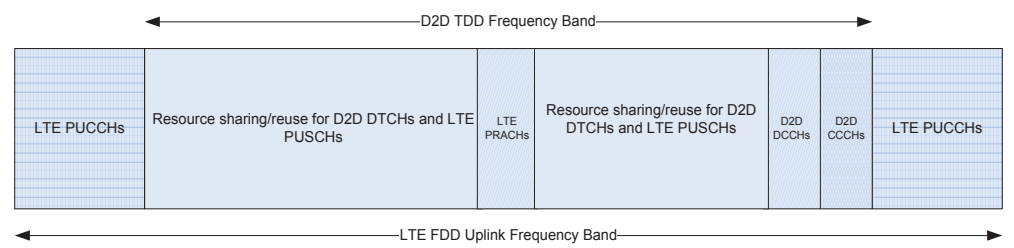

Fig. 3. Illustration of frequency resource sharing between D2D and LTE systems.

Furthermore, the following assumptions are used in our design.

1) The cellular network and D2D subsystem use FDD and Time Division Duplexing (TDD) respectively whilst D2D users will share the UL spectrum of cellular system with the cellular UEs. Comparing with other duplex modes, the benefits by using this mode include that only one transceiver is needed for D2D devices and channel reciprocity and asymmetric data transmission can be realized which are in-built traits of a TDD system. Another, since the cellular system works in the FDD mode, it is possible to dynamically switch shared spectrums between UL and DL to adapt to different UL/DL interference.

2) The following description is based on the assumption that the cellular system is LTE as an example. However, the idea can also be used in other Orthogonal Frequency Division Multiple Access (OFDMA), Single-Carrier Frequency Division Multiple Access (SC-FDMA) or FDMA systems.

3) The proposed scheme can be easily adapted to the mixed scenario where the cellular system uses TDD.

Based on the above descriptions, the complete handshake mechanism is described as follows.

Step1: The eNB broadcasts the reserved resource information on $\mathrm{CCCH}$ and DCCH along with the TTI characteristic on LTE BCH to all UEs in the hybrid network.

Step2: The Tx_D_UE transmits RTS to announce its data transmission request, which is also a competition announcement to the neighboring D2D pairs. The identity (ID) of the Tx_D_UE and Rx_D_UE should also be included in RTS signaling.

Step3: The Rx D U UE will respond with CTS if RTS is received without any collision with other D2D UEs. Otherwise no CTS will be sent back to the Tx_D_UE. In CTS signaling, the ID of the Tx D UE and Rx D UE should also be included.

Step4: Receiving CTS from the Rx_D_UE correctly means Tx_D_UE has obtained the priority to send data in the corresponding TTI. Else if no CTS is received, Tx D UE will back off for some random time $T_{0}$ before a new data transmission request. When a handshake procedure stops because of no CTS response, $T_{0}$ will be augmented, otherwise it will be decreased. In this way, the intensity of the competition through RTS and CTS can be controlled even if there are a large number of D2D UEs in a small area. To keep the rigid time relationship, $T_{0}$ should be defined as $T_{0}=2 T_{\mathrm{t}}+N T_{\Delta}$, where $T_{\mathrm{t}}$ means the duration of a TTI, $N$ denotes a random integer and $T_{\Delta}$ is a fixed period. In this expression, two TTIs are used to avoid the possible collisions at the CTS signaling thus leading to high spectrum efficiency.

Step5: Receiving CTS from the Rx_D_UE successfully, Tx_D_UE receives UL MAP from 
210 Xu et al.: Device-to-Device Communication Underlaying Cellular Networks: Connection Establishment and Interference

PDCCH simultaneously. Using 3 TTIs to decode the UL resource and prepare for the data transmission, Tx_D_UE sends the DataCtrl signaling on DCCH on the fourth TTI and D2D data on the DTCH which is a reusing channel with the PUSCH of the LTE system. The DataCtrl signaling associates with the data transmission, such as the data format, coding and modulation modes, resource assignment and so on.

Step6: On reception of D2D data and DataCtrl signaling, the Rx_D_UE needs 4 TTIs to process them and then sends ACK/NAK back to Tx_D_UE on DCCH to feed back the status of data reception, which will be used for data retransmission by Tx D UE. Note that different from a regular LTE system, in this step an additional TTI is used to allow the Rx_D_UE to process the received data. By doing so, DataCtrl and ACK/NACK signaling can appear alternately on the $\mathrm{DCCH}$ and thus avoiding collisions.

The detailed time relationship of a single and multiple D2D communication are shown in Fig. 4 and 5, respectively.
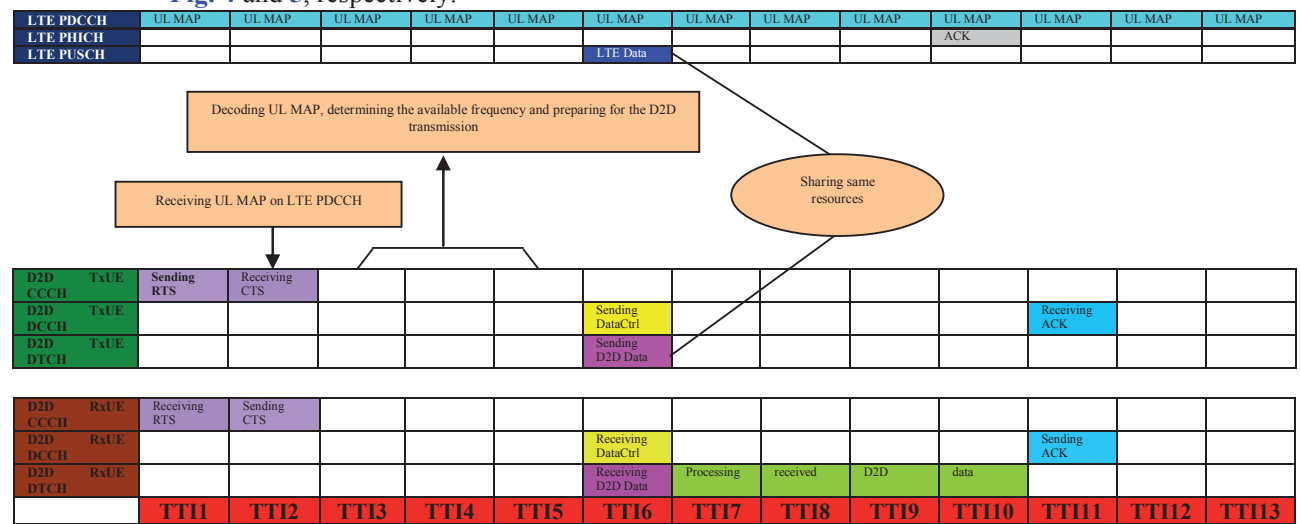

Fig. 4. A complete time relationship of single D2D transmission.
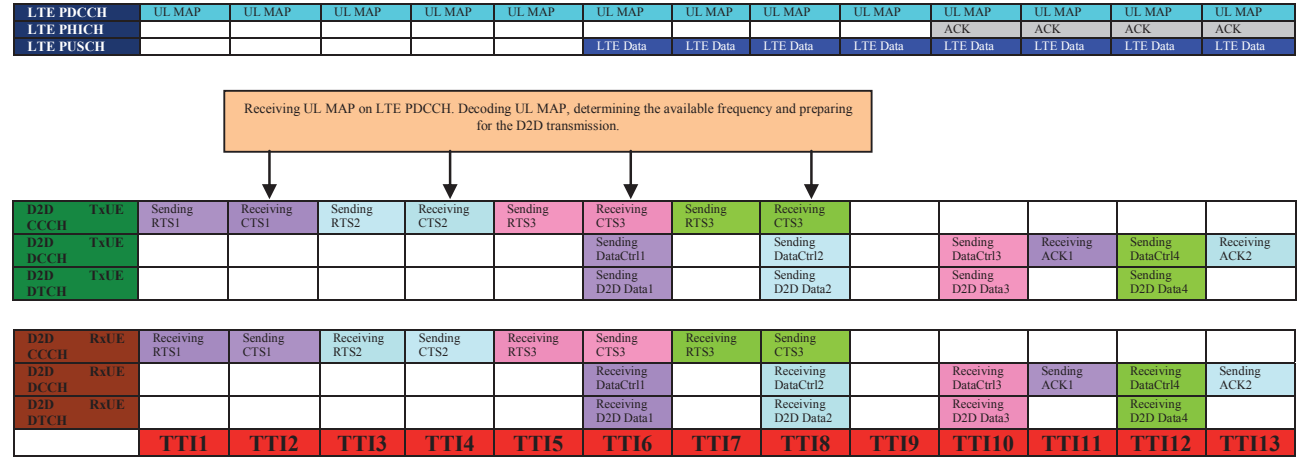

Fig. 5. A complete time relationship of multiple D2D transmission. 
As a summary, the proposed transmission mechanism has the following traits.

1) The D2D signaling and data transmission follow the same frame and timing structure as the UL operation of the cellular network.

2) Under the proposed scheme, D2D and cellular communication share the same frequency to send data thus improving the spectrum efficiency and avoiding transmission collisions.

\section{Mechanisms of Near-Far Interference Avoidance in Cellular UL Spectrums}

Interference management in a hybrid network is a critical issue in that the interference from D2D links is expected to reduce the cellular capacity and efficiency. In the LTE UL, a cellular UE sends data after several TTIs on receipt of resource allocation information from the eNB. Therefore, D2D UE may finish its RRM by using the related cellular information within these several milliseconds [13]. Additionally, the UL is underutilized by the cellular operators comparing to DL [14]. Thereby, most research of D2D transmission focuses on the scenario that D2D users use the uplink spectrum of an LTE system [9][13][14][15][16][17]. However, working autonomously and fully sharing frequency resources with cellular users, D2D UEs may sustain near-far interference from cellular terminals. The addressed problems are illustrated in Fig. 6, where cellular UE1 may impose serious interference to D2D transmission if they are sharing the same resource. Whereas the interference from UE2 can be negligible since the long distance between the D2D pair and UE2. To arrange the dedicated resource for the cellular system and D2D users is an intuitive solution but leads to inefficient utilization of the available resources and low system throughput.

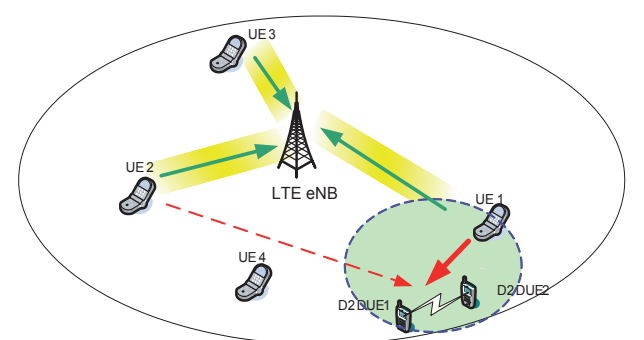

Fig. 6. An example of near-far interference in the LTE uplink spectrum.

In our work, we consider the D2D UEs reuse the whole uplink frequency bands with cellular UEs in an LTE FDD system and the proposed mechanism in Section 2 is used to establish and maintain D2D transmission. When UL spectrums are used, the victim cellular device is the eNB. Given that fractional power control in LTE can be used on D2D communication, the interference from a D2D user to the eNB can be avoided efficiently according to the existing study. Consequently, the interference from cellular users to D2D transmission will be addressed. In this section, we propose a novel scheme to realize UL resources sharing meanwhile avoiding near-far interference to D2D transmission in a hybrid network. By 
monitoring the D2D channels ( $\mathrm{CCCH}$ and $\mathrm{DCCH})$, an eNB will identify the 'near-far-risk' cellular users and broadcast the information on their allocated resources. Based on this knowledge, D2D devices can proactively perform RRM to avoid the near-far interference from cellular UEs. Different from the previous work [9][13][14][15][16][17], by utilizing the proposed scheme, the C-RNTI of D2D UEs is not always necessary and the interference from the neighboring cell can be suppressed as well.

In our work, autonomous D2D transmission is assumed which means that each D2D pair autonomously determines the resource allocation by using some useful knowledge from the eNB. Comparing with the eNB completely controlled D2D communication, it benefits from lower control signaling overhead and less modification to the existing cellular infrastructure. Since the D2D subsystem is underlying the LTE network, the eNB allocates the resources to cellular UEs in a dynamic way, which means that the cellular UL resource allocation can not be predicted accurately by the accumulated knowledge. Therefore, blind interference avoidance methods are difficult to work if there is no tight time correlation among resource allocation of cellular users.

Denote C_UE and D_UE a cellular UE and a D2D UE separately, the proposed scheme to share cellular UL resources in a hybrid system is presented in detail as follows.

Step 1: At the beginning of D2D transmission, eNB broadcasts the reserved resource including time and frequency of D2D CCCH and DCCH in the broadcasting channel such that all C UEs and D UEs have the idea of the information on D2D channels.

Step 2: After that, cellular UEs periodically listen to the signaling on $\mathrm{CCCH}$ and DCCH from D2D UEs to ensure whether D2D UEs are close to them.

Based on the explanation of the handshake process during the D2D communication, control signaling is always transmitted on the $\mathrm{CCCH}$ and $\mathrm{DCCH}$ which is a dedicated channel for D2D communication. Hence, the sensed signals from D2D channels are assumed to be reliable.

Here a signal-to-interference-plus-noise ratio (SINR) threshold and a listening period are needed to identify the adjacent D2D UEs accurately. According to the peculiarity of the cellular system, two criteria are proposed for the cellular users.

- Criterion I: Cellular UEs only monitor the power of D2D channels regularly without decoding the information they received.

- Criterion II: Cellular UEs decode the information obtained from D2D channels to find the IDs of neighboring D2D UEs and then report their IDs to the eNB in the next step. Note that, to utilize Criterion II, the information such as Cell ID and C-RNTI should be included in the handshake procedure. C-RNTI is assigned by the eNB on the PDCCH during the Random Access Response to identify the UE uniquely [18].

Step 3: Once a C UE senses an SINR beyond the predefined threshold, it will report the measurement result to the eNB in the earliest available UL time slot in a dedicated channel. According to the two different criteria in Step 2, two report formats will be utilized.

- Format I: Cellular users report their position information to the eNB. In this format, it is required that $\mathrm{C}$ UEs and $\mathrm{D}$ UEs can obtain their position information. Considering that mounting a Global Positioning System (GPS) device in a cell phone is so popular nowadays we think this format is feasible.

- Format II: Cellular users report the ID of the detected D2D UE to its eNB.

Two examples are given in Table $\mathbf{1}$ and $\mathbf{2}$ according to Format I and Format II, respectively. From Table 1 we can observe that C_UE1 with the position of (X1, Y1) and C_UE2 with the 
position of $(\mathrm{X} 2, \mathrm{Y} 2)$ are near to the $\mathrm{D} 2 \mathrm{D}$ pairs such that they will impose strong interference to the D2D communication if the same UL resource is reused. Different from Table 1 where the IDs of D2D users can not be obtained, eNB is able to derive directly that C_UE1 is adjacent to D_UE1 and D_UE2 by using Format II.

Table 1. Interference Map Based on Format I

Table 2. Interference Map Based on Format II

\begin{tabular}{|c|c|}
\hline ID of C_UE & Position of C_UE \\
\hline C_UE1 & $(\mathrm{X} 1, \mathrm{Y} 1)$ \\
\hline C_UE2 & $(\mathrm{X} 2, \mathrm{Y} 2)$ \\
\hline$\ldots$ & $\ldots$ \\
\hline
\end{tabular}

\begin{tabular}{|c|c|}
\hline ID of C_UE & ID of D_UE \\
\hline \multirow{2}{*}{ C_UE1 } & D_UE1 \\
\cline { 2 - 2 } & D_UE2 \\
\hline C_UE2 & D_UE3 \\
\hline$\ldots$ & $\ldots$ \\
\hline
\end{tabular}

Note that the reported measurement results can be Time Division Multiplex (TDM), Frequency Division Multiplex (FDM) or Code Division Multiplex (CDM) on the dedicated channel and only the cellular UEs receiving higher power from D2D channels will report to the eNB thereby reducing the signaling overhead.

In a scenario of multiple cellular networks, inter-cell interference from the neighboring cell may occur especially when D2D UEs are at the edge of the given cell. To overcome such interference, a TDM scheme is proposed. When a cellular UE measures the power from the D2D channels beyond the pre-defined threshold, it should report to its anchored eNB. Then by checking the D_UE's ID, an eNB can determin if this D_UE is under its control. If it is not true, it means that inter-cell interference occurs and therefore the eNB stops scheduling these reporting cellular UEs during this D2D transmission. By using this mechanism, the inter-cell interference from the neighboring cell can be avoided effectively.

Step 4: According to the received measurement results, the eNB identifies the 'near-far-risk' cellular UEs. For a 'near-far-risk' UE, its received SINR from the D2D channels is beyond the threshold therefore having high probability to interfere with its neighboring D2D users. Thus, the eNB broadcasts the allocated resources for all the 'near-far-risk' cellular UEs in the dedicated channel. Based on the two different report formats in Step 3, the broadcasting information can be classified as follows.

- Broadcasting information $\mathbf{I}$ : The eNB broadcasts the position information and allocated resources of 'near-far-risk' cellular users, thus D2D users can use such information to avoid reusing those resources at the specific location. To implement this approach, D2D UEs need to know the position of their own. As a result, a D_UE can reuse the resource with a distant C_UE and avoid interference from a near cellular user. Here, no D2D RNTI is needed so security and reusing efficiency is guaranteed.

- Broadcasting information II: Prohibited frequency resources and IDs of D2D UEs are sent by the eNB.

Table 3 and 4 present examples to explain this step. From Table 3 all D2D UEs know that the allocated resource for the C_UE with the position (X1, Y1) is RB3, such that after the simple calculation, the D2D UEs whose positions are near to (X1, Y1) will not use the RB3 to implement their D2D transmission. However, D2D UEs can obtain the prohibited resource directly from Table 4. For example, D_UE 1 can know that RB3 and RB6 are allocated to the adjacent cellular UEs so D_UE 1 will avoid reusing these two RBs to mitigate the near-far interference. 
214 Xu et al.: Device-to-Device Communication Underlaying Cellular Networks: Connection Establishment and Interference

Table 3. Broadcasting information I

\begin{tabular}{|c|c|}
\hline Position of C_UE & \multicolumn{2}{|c|}{ Allocated Resources } \\
\hline$(\mathrm{X} 1, \mathrm{Y} 1)$ & $\mathrm{RB} 3$ \\
\hline$(\mathrm{X} 2, \mathrm{Y} 2)$ & \multicolumn{2}{|c|}{ RB6 } \\
\hline$\ldots$ & $\ldots$ \\
\hline
\end{tabular}

Table 4. Broadcasting information II

\begin{tabular}{|c|c|}
\hline ID of D_UE & Prohibited Resources \\
\hline \multirow{2}{*}{ D_UE 1 } & RB3 \\
\cline { 2 - 2 } & \multicolumn{2}{|c|}{ RB6 } \\
\hline D_UE 2 & RB8 \\
\hline$\ldots$ & $\ldots$ \\
\hline
\end{tabular}

Step 5: On receipt of the broadcasting messages in Step 4, D2D UEs autonomously perform RRM to mitigate near-far interference from cellular UEs.

Note that the information in Step 4 should be broadcasted in advance of 4 TTIs with the cellular UL grant information. By doing so, D2D signaling and data transmission can follow the same frame structure as the UL of the cellular network. Therefore the time relationship could be well defined to allow D2D UEs know that which resource will be utilized through the handshake and competition procedure at the specific time.

The complete procedure of our proposed near-far interference cancellation mechanism in an LTE FDD system is illustrated in Fig. 7.

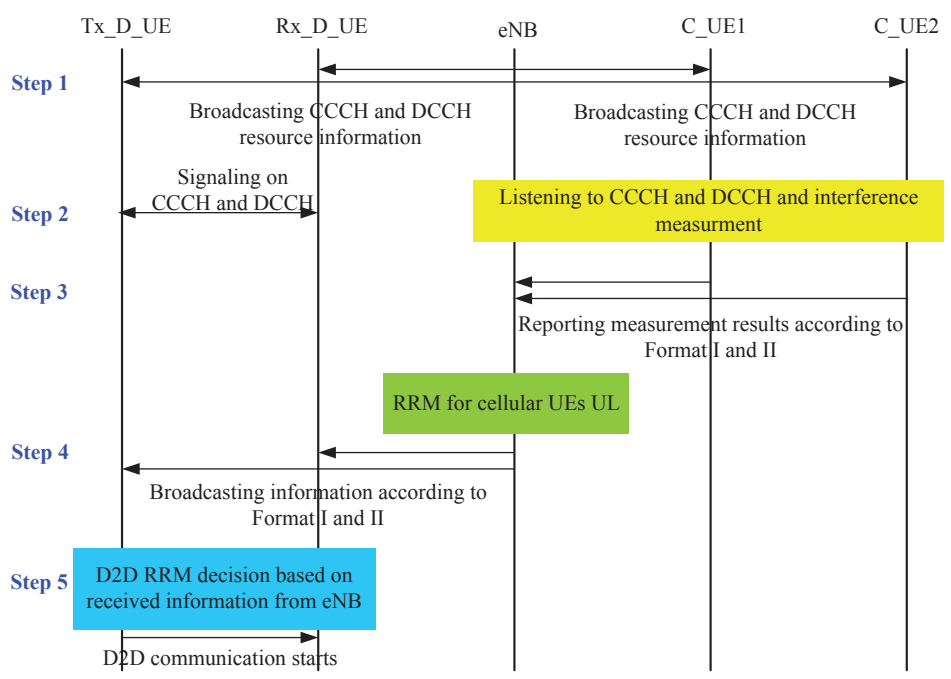

Fig. 7. Procedures of the proposed method to share LTE UL spectrums.

\section{Effective Labeled Time Slots Based D2D Transmission in Cellular Downlink Spectrums}

In an LTE system, different scheduling mechanisms enable unparallel opportunities for D2D users using UL and DL of a cellular network. In the LTE system, the UL resource is underutilized by the cellular operators comparing to DL [14] and several milliseconds are 
reserved for the UE after receiving the resource allocation information from the eNB, thus making the RRM of D2D systems feasible. On the contrary, in an LTE like cellular network, since continuous control signaling and data are transmitted and fast scheduling are used in the DL spectrum, it is very difficult for the D2D transmission to share DL spectrums with cellular users. This problem can be illustrated in Fig. 8. In the first TTI, by decoding the control part of cellular DL signaling, D2D UEs may know which resources are available for them to perform D2D communication. However, in the following second TTI, before D2D UEs can finish resource allocation for D2D communication, the available resources for D2D UEs changed.

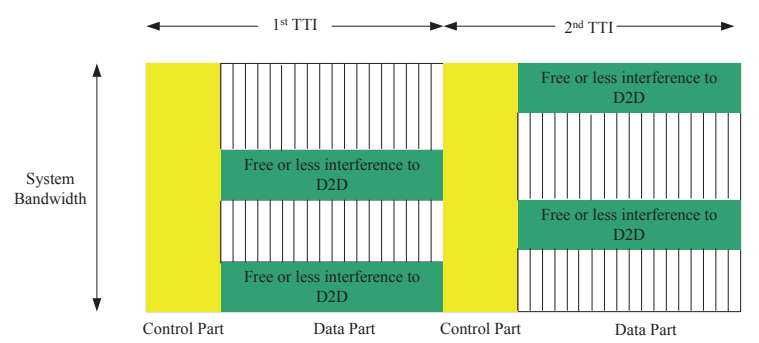

Fig. 8. An example of fast scheduling in the LTE DL transmission.

Other than the fast resource allocation, possible near-far interference is another serious problem when DL spectrums are reused and this interference is presented in Fig. 9. From this figure we observe that the D2D UE1 and D2D UE2 may impose significant interference to the cellular UE1 since the short distance between them. So the key problem for sharing DL spectrums of a hybrid system is to avoid interference to nearby cellular UEs.

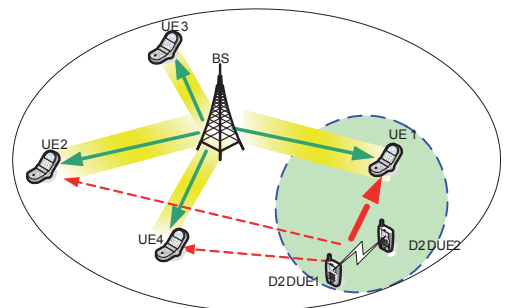

Fig. 9. Near-far interference when sharing cellular downlink spectrums.

In this section, we propose a novel scheme to realize DL resources sharing meanwhile avoiding near-far interference from D2D transmission by using labeled time slots. By classifying the cellular users as 'non-near-far-risk' cellular users and 'near-far-risk' cellular users, the eNB disparts the whole system resource as 'shared time slots' and 'cellular dedicated time slots'. As a result, the eNB will schedule 'near-far-risk' cellular users in 'cellular dedicated time slots' only but the 'non-near-far-risk' cellular users in both kinds of time slots while D2D pairs will share 'shared time slots' with the 'non-near-far-risk' cellular users. 
Similar to Section 3, the handshake and competition mechanisms described in Section 2 are used and we assume the sensed signals from D2D channels are reliable. Moreover, D2D users operate in an underlay mode which means that the eNB controls D2D users loosely by sending limited assisting information to facilitate D2D RRM. Thus the proposed scheme to share cellular DL resource in a hybrid system is presented in detail as follows.

Step 1: At the beginning of D2D transmission, the eNB broadcasts the reserved resource of D2D CCCH and DCCH in the LTE BCH such that all cellular UEs and D2D pairs know the information of $\mathrm{D} 2 \mathrm{D}$ channels.

Step 2: After that, cellular UEs periodically listen to the signaling on $\mathrm{CCCH}$ and DCCH to ensure whether D2D UEs are near to them. Here an SINR threshold and a listening period are needed to identify the adjacent D2D UEs accurately.

Step 3: Once a cellular UE whose sensed SINR from D2D channels is beyond the predefined threshold, it will report the measurement result to its eNB in the earliest available UL time slot in a dedicated channel. Here, a 1-bit result can be used to show whether there are D2D pairs nearby or multiple bits can also be used to indicate different interference levels.

Step 4: According to the received measurement results, the eNB classifies all cellular UEs into two groups, namely, 'non-near-far-risk' UEs and 'near-far-risk' UEs. A 'non-near-far-risk' UE sensed SINR to be below the known threshold so that D2D users do not impose interference to it despite sharing the same resource. Nevertheless, a 'near-far-risk' UE whose received SINR from the D2D channels is beyond the threshold has high probability to be interfered by the adjacent D2D users. Correspondingly, the eNB divides the time resources in one radio frame into two groups which are 'cellular dedicated time slots' and 'shared time slots'. All cellular UEs can be scheduled in the 'cellular dedicated time slots' whereas no D2D UEs is allowed to send data in these time slots. Nevertheless, D2D communication may occur in the 'shared time slots' with 'non-near-far-risk' cellular UEs.

To implement our approach, all cellular UEs will be identified as 'non-near-far-risk' UEs in the initial communication stage and the eNB will modify its scheduling decision according to the following interference report. Additionally, the allocated time slots which may be continuous or not should depend on the traffic of cellular and D2D UEs and be updated from time to time. Furthermore, D2D UEs are allowed to require the eNB to allocate more or less 'shared time slots'.

Step 5: The eNB will broadcast the available time slots allocation information (namely the 'shared time slots' information) for all D2D UEs in advance.

Step 6: Obtaining the available DL resource information, D2D transmission may occur in the 'shared time slots' along with the 'non-near-far-risk' UEs. In addition, the eNB will schedule 'near-far-risk' cellular users in 'cellular dedicated time slots' only. Note that multiple D2D pairs can share resources spatially by using the contention based RTS/CTS procedure aforementioned in Section 2.

The complete procedure of our proposed labeled time slots based mechanism in an LTE FDD system can be summarized in Fig. 10. 


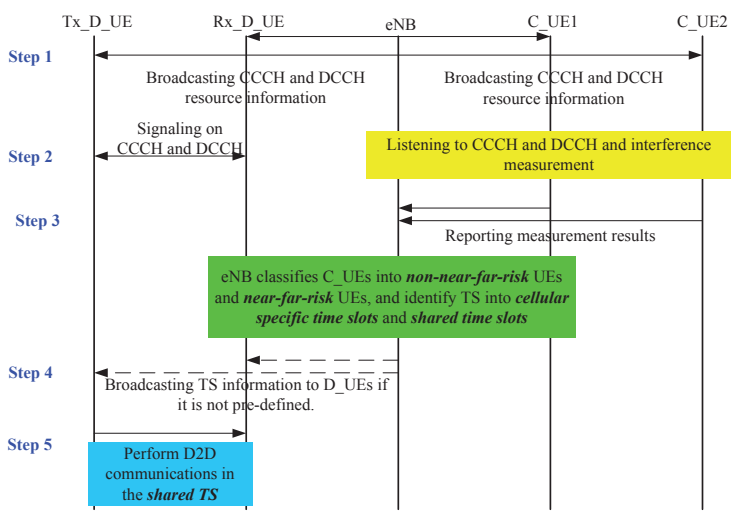

Fig. 10. Procedures of the proposed method to share cellular DL spectrum.

In that D2D communication will continue for some periods and it is unnecessary for cellular UEs to periodically monitor D2D channels during this interval, here a power saving scheme is proposed which is illustrated in Fig. 11. Two periods, namely, interference-avoidance period and interference-clear period are defined by the eNB and are broadcasted to cellular UEs in advance. During the interference-avoidance period, D2D UEs are active and cellular UEs close to them do not listen to D2D interference periodically. An interference-clear period is at the end of the interference-avoidance period, during which, cellular UEs sense D2D channels and send 'near-far-risk' indication if there is D2D signaling in the most recent interference-clear period. As shown in Fig. 11, since no D2D signaling is detected at the end of an interference-clear period, an interference-avoidance period ends and cellular UEs continue to monitor D2D channels periodically.

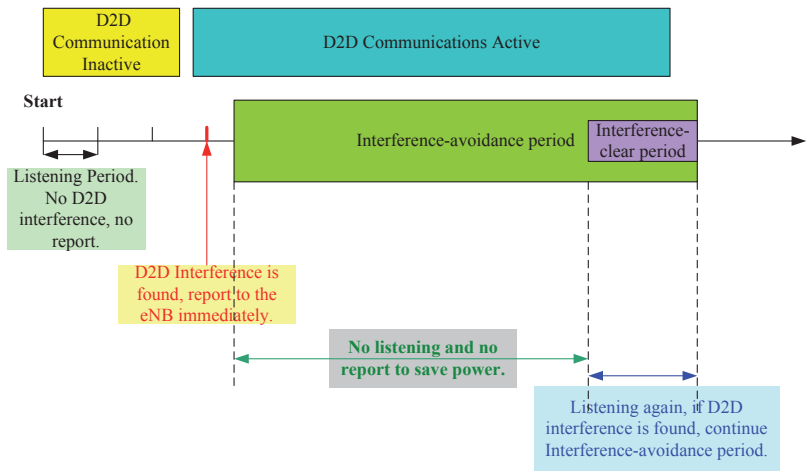

Fig. 11. Power saving scheme for cellular UEs.

\section{Simulation and Performance Analysis}


In this section, simulation results are shown to evaluate the performance of a hybrid system by using our ideas for both UL and DL resources sharing.

\subsection{Simulation Parameters}

The topology of the hybrid system will be modeled in a fashion way where D2D users are distributed in a randomly placed cluster with a radius of $10 \mathrm{~m}$ and cellular users are also dropped uniformly through the cell with a radius of $300 \mathrm{~m}$. Such a topology is more realistic in modeling urban environments [17]. There are altogether 50 D2D users placed in the system with regards to the number of cellular UEs changing from 50,100, 200 to 2000. Considering the short distance between a D2D pair and the large radius of the cellular system, a simple power control scheme is utilized by letting the transmission power of Tx_D_UE $15 \mathrm{~dB}$ lower than that of cellular users. The used system bandwidth is $1.4 \mathrm{MHz}$, i.e., 6 RBs altogether. By reserving $2 \mathrm{RBs}$ for the $\mathrm{CCCH}$ and $\mathrm{DCCH}$ of a $\mathrm{D} 2 \mathrm{D}$ subsystem, the left $4 \mathrm{RBs}$ are available to transmit data for both networks. The adopted threshold by cellular UEs to determine if D2D users are adjacent to them is SINR which is received from $\mathrm{CCCH}$ and DCCH.

The other parameters are set up according to [19] and are presented in Table 5.

Table 5. Parameters for simulation

\begin{tabular}{|l|l|}
\hline Cell radius & $300 \mathrm{~m}$ \\
\hline Distance of two D2D UEs & $10 \mathrm{~m}$ \\
\hline Noise Power Density & $-174 \mathrm{dBm} / \mathrm{Hz}$ \\
\hline Noise figure & $5 \mathrm{~dB}$ \\
\hline Cellular link pathloss (PL) model & $\begin{array}{l}\mathrm{PL}=128.1+37.6 * \log 10(\mathrm{R}), \mathrm{R} \text { is the } \\
\text { transmitter-receiver separation in kilometers [6] }\end{array}$ \\
\hline D2D link pathloss model & $\begin{array}{l}\mathrm{PL}=127+30^{*} \log 10(\mathrm{R}), \quad \mathrm{R} \text { th } \\
\text { transmitter-receiver separation in kilometers [6] }\end{array}$ \\
\hline $\begin{array}{l}\text { Maximum transmission power of } \\
\text { eNB, C_UE and D_UE }\end{array}$ & $46 \mathrm{dBm}, 24 \mathrm{dBm}$ and 9dBm \\
\hline RB bandwidth & $180 \mathrm{kHz}$ \\
\hline Carrier frequency & $2000 \mathrm{MHz}$ \\
\hline TTI length & $1 \mathrm{~ms}$ \\
\hline System bandwidth & $1.4 \mathrm{MHz}$ \\
\hline
\end{tabular}

\subsection{Simulation Results and Discussions}

Fig. 12 and 13 firstly presents the system average throughput vs. the threshold for reusing UL and DL spectrums, respectively. In order to show the effectiveness of our method, we also consider different densities of mobile users in sharing UL and DL scenarios. To evaluate our method, the curve of cellular system average throughput when no D2D users present is used as the benchmark. We also plot the cellular system average throughput when D2D users exist but no any method is employed to mitigate the interference. In order to prove the performance of our method, the cellular system average throughput and average throughput of the cellular users plus D2D UEs are compared in each figure. For sharing DL spectrums, a power control scheme is typical to suppress the interference from D2D users to the cellular UEs. We compare the performance of the proposed mechanism with the power control scheme and the results are presented in Fig. 13. To be fair, the transmission power of a D2D device is set up to keep the same average throughput of cellular users by exploiting our approach and the power control 
method.

Four observations can be made from Fig. 12 and Fig. 13 under different densities of mobile users. First of all, D2D transmission imposes significant interference to the cellular users when sharing DL spectrums and such interference can degrade the system performance greatly. For reusing UL resources, cellular UEs also interfere with D2D transmission remarkably. By applying the proposed mechanism, the system average throughput is increased substantially under different mobile user densities. Secondly, the throughput is highly related to the adopted threshold. We can observe that with the increase of the adopted threshold, the curves with and without interference avoidance gradually converges and this is especially remarkable when sharing UL spectrums. While the used threshold is increased, the throughput is reduced. This result is feasible and can be explained as follows. For sharing UL resources, when a higher threshold is adopted, few cellular UEs report to the eNB as 'near-far-risk' UEs thereby imposing much more interference on the D2D subsystem. However, a lower threshold means more cellular devices are determined to be the interferers of the D2D subsystem. As a result, the eNB and D2D UEs need to deal with more information and the system complexity is increased. Consequently, a tradeoff should be made to balance the system performance and complexity. Similarly, when DL spectrums are reused, a higher threshold means more D2D devices are able to use the 'cellular dedicated time slots' thereby imposing interference to the cellular terminals to degrade their performance. Thirdly, when reusing UL resources, with the reduction of the cellular users, the system throughput increases significantly due to the decrease of the interference incurred by the cellular devices. Finally, from Fig. 13 we conclude that a gain of system sum throughput can be obtained by using the proposed scheme comparing with the traditional power control method under three different densities of mobile users. This result is straightforward that by reducing the transmission power of D2D pairs, the average throughput of the cellular users is kept invariable but the throughput of the D2D subsystem will decrease thus leading to the decrement of the sum throughput.

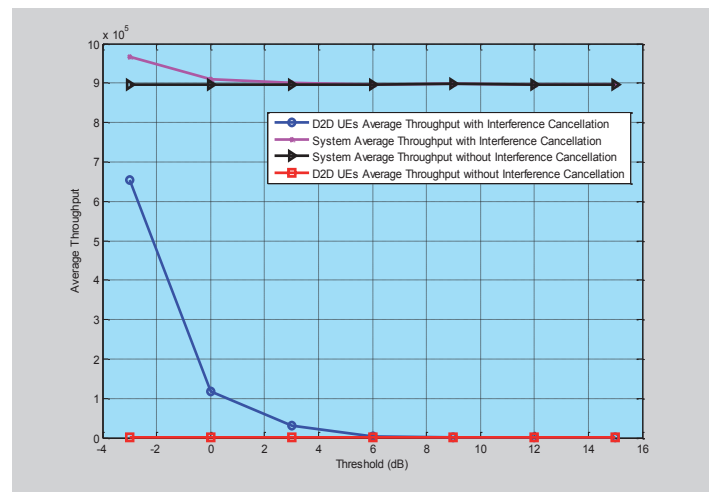

Fig. 12. (a) 200 cellular UEs vs. 50 D2D UEs 


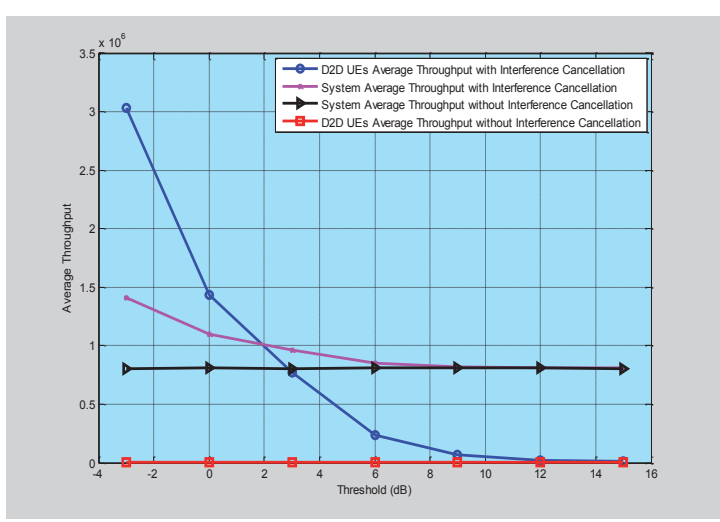

Fig.12. (b) 100 cellular UEs vs. 50 D2D UEs

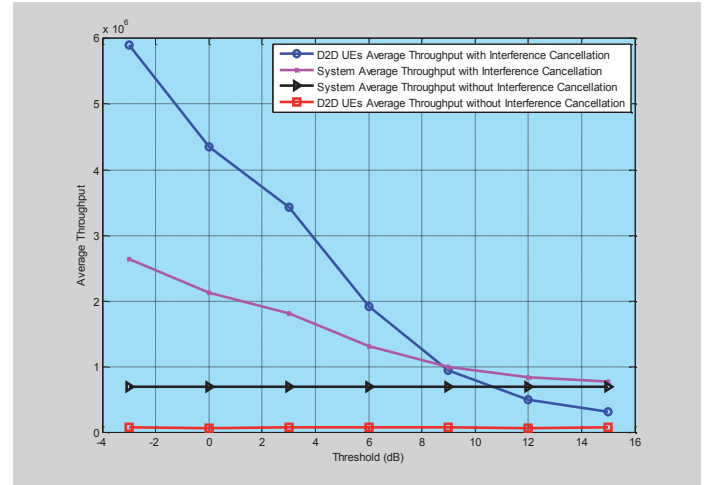

Fig.12. (c) 50 cellular UEs vs. 50 D2D UEs

Fig. 12. System average throughput vs. the threshold when reusing UL spectrums under different densities of mobile users. 


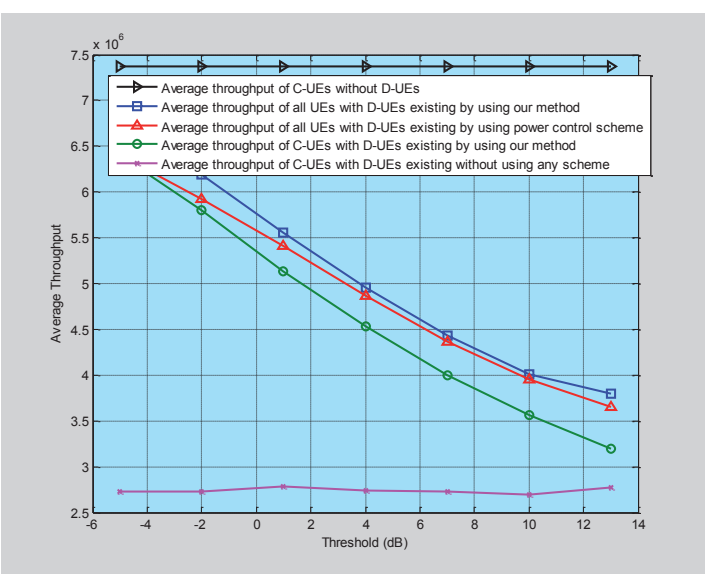

Fig. 13. (a) 2000 cellular UEs vs. 50 D2D UEs

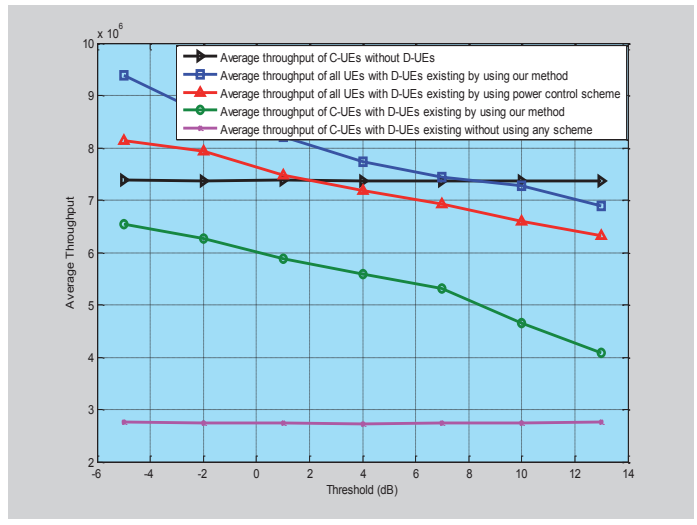

Fig. 13. (b) 200 cellular UEs vs. 50 D2D Ues 


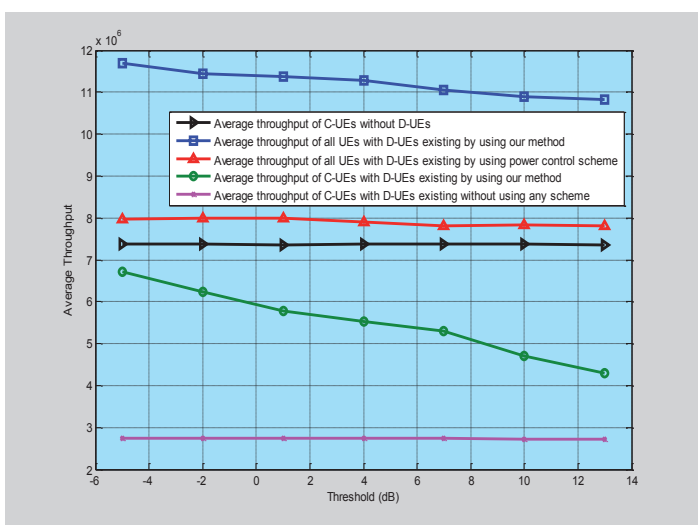

Fig. 13. (c) 50 cellular UEs vs. 50 D2D Ues

Fig. 13. System average throughput vs. the threshold when reusing DL spectrums under different densities of mobile users.

We further investigate our approaches when considering the different ratios of a transmission duration of the D2D subsystem and the cellular subsystem as 1:1, 1:2, 1:3 and 1:4 respectively. The simulation results corresponding to reusing UL and DL spectrums are seperately illustrated in Fig. 14 and 15. The considered system consists of 50 C_UEs plus 50 D_UEs for sharing UL spectrums whilst 200 C_UEs plus 50 D_UEs when reusing DL resources. The presented simulations prove the effctiveness of the proposed schemes in shaing UL and DL spectrums under different transmission duration ratios settings.

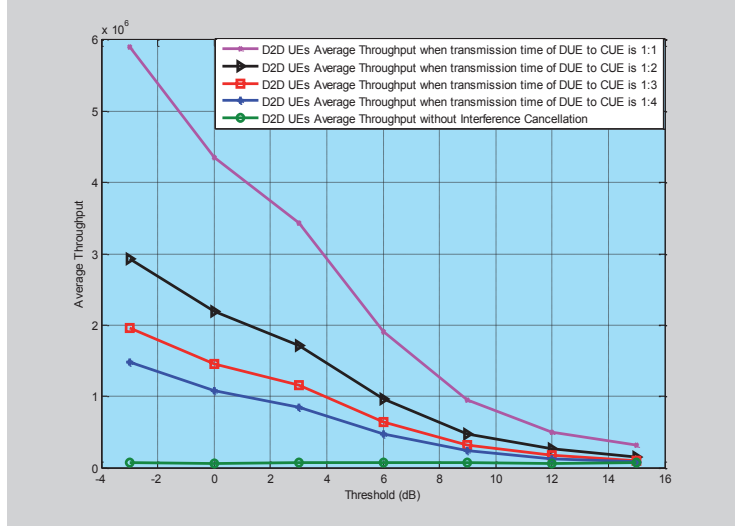

Fig. 14. System average throughput vs. the threshold when reusing UL spectrums under different transmission duration ratios. 


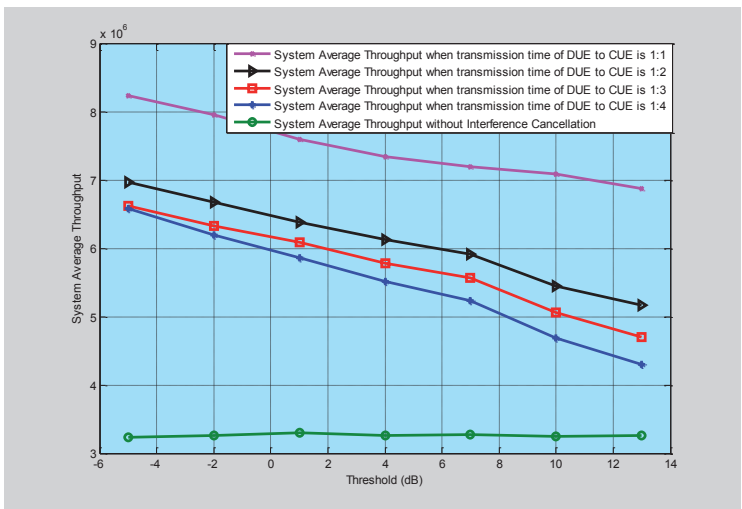

Fig. 15. System average throughput vs. the threshold when reusing DL spectrums under different transmission duration ratios.

Fig. 16 and 17 show the effects of our interference cancellation mechanisms when changing the distance of two D2D users when sharing UL and DL spectrums respectively. The considered hybrid system consists of $50 \mathrm{C}$ UEs plus $50 \mathrm{D}$ UEs for sharing UL spectrums and 200 C_UEs plus 50 D_UEs for sharing DL $\bar{L}$ resources with the adopted threshold of $3 \mathrm{~dB}$. For sharing UL spectrums, distance between two users is vital in a D2D subsystem. When two D2D users are very close, high throughput can still be obtained in that the close D2D pair can effectively counteract the interference from the cellular system. However, distance of two D2D users is not so important to avoid the interference to the cellular UEs but improving the system sum throughput when these two subsystems reuse DL spectrums.

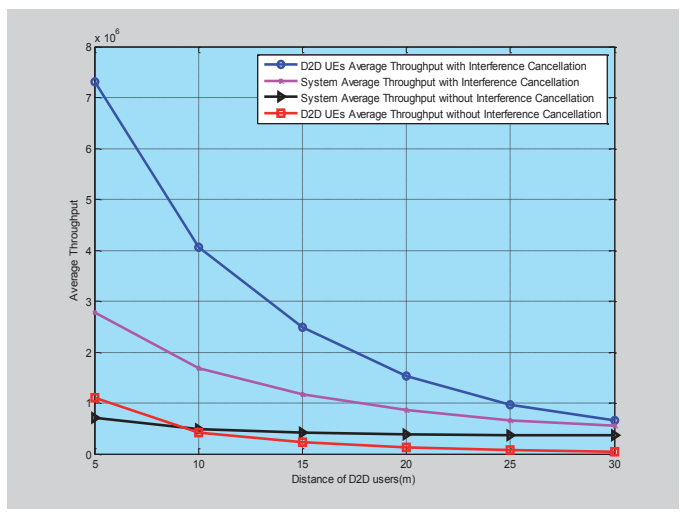

Fig. 16. System average throughput vs. distance of D2D users when reusing UL spectrums 


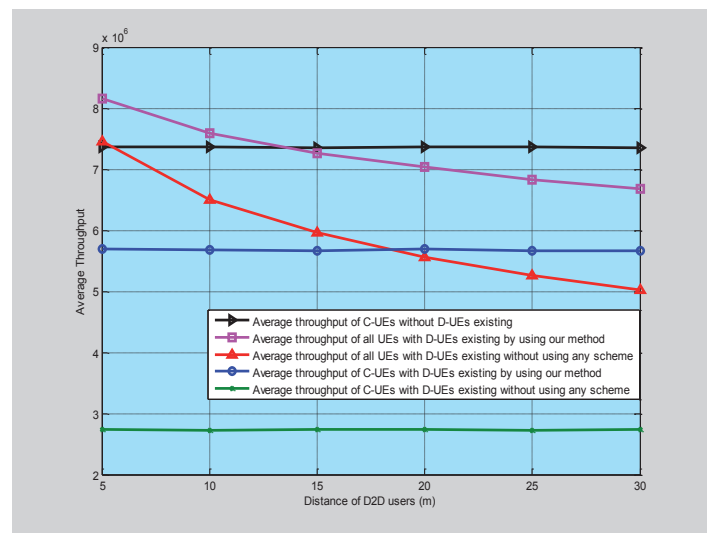

Fig. 17. System average throughput vs. distance of D2D users when reusing DL spectrums.

To evaluate our approach to cancel UL interference, the cumulative distribution function (CDF) curves of received interference from the cellular subsystem are collected and the simulation results are plotted in Fig. 18. Here, we use the population of 200 C_UEs plus 50 D UEs with the adopted interference cancellation threshold of $-3 \mathrm{~dB}, 2 \mathrm{~dB}$ and $7 \mathrm{~dB}$, respectively. From this figure we can conclude that the interference is mitigated significantly after using our mechanism comparing with no approach is implemented to avoid such interference. Furthermore, the efficacy becomes better with the decrement of the threshold. This is due to the fact that a lower threshold results in avoiding more interference to the D2D subsystem.

To get insight of the proposed mechanism to reuse DL spectrums with LTE systems, the CDF curves of received interference by cellular subsystem are presented in Fig. 19 with 2000 C UEs plus 50 D UEs and the adopted threshold of $3 \mathrm{~dB}$ and $6 \mathrm{~dB}$ separately. To investigate the performance improvement by utilizing the proposed approach, we also compare the simulations of our method with the power control scheme. The same method is used to set up the transmission power of the D2D UEs as Fig. 13. From this figure we observe that the proposed mechanism outperforms the typical power control method and such near-far interference is mitigated greatly after using our approach. We also conclude that lower threshold leads to a better performance because more interfernce is detected and suppressed. 


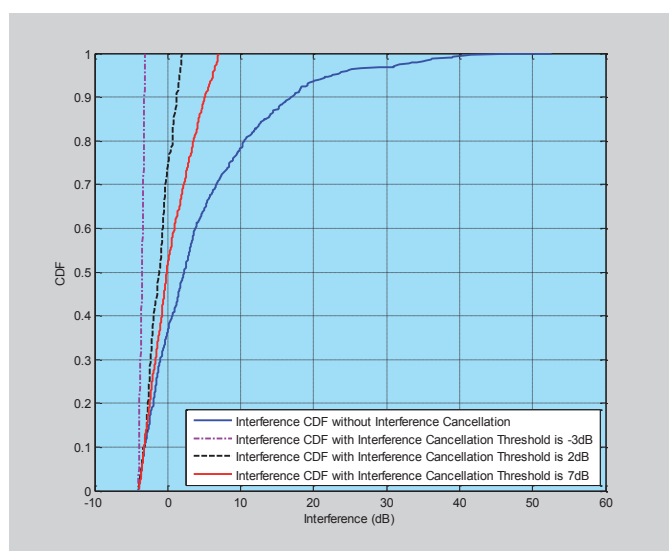

Fig. 18. CDF of near-far interference from cellular transmission when reusing UL spectrums.

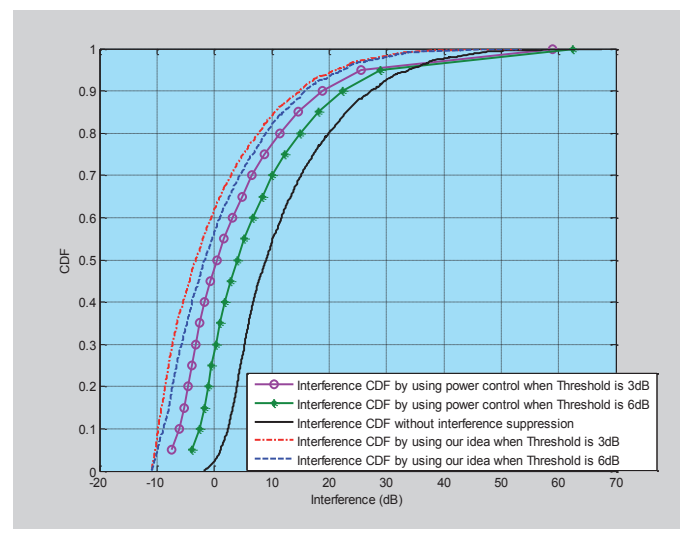

Fig. 19. CDF of near-far interference from D2D transmission when reusing DL spectrums.

\section{Conclusions}

In this article, we firstly propose the effective mechanism to establish and maintain D2D communication in a hybrid network by using the SSARC scheme. Based on this proposed handshake procedure, the approaches to share UL and DL spectrums with an LTE FDD network in a hybrid system are addressed respectively in this paper. By tracking the near-far interference and identify the interfering cellular users, the UL frequency bands can be used efficiently meanwhile avoiding the harmful interference from cellular networks to D2D 
transmission. To efficiently share the DL spectrums, the time slots are labeled and the cellular users are identified so that the harmful interference from D2D transmission to cellular networks can be avoided as well. The proposed schemes are evaluated in a local area cellular network. The extensive simulations prove that better performance can be obtained by comparing with the conventional power control mechanism.

\section{References}

[1] K. Doppler, M. Rinne, C. Wijting, C. Ribeiro, and K. Hugl, "Device-to-device communication as an underlay to LTE-advanced networks," IEEE Communications Magazine, vol. 47, no. 12, pp. 42-49, 2009. Article (CrossRef Link)

[2] K. Doppler, M. P. Rinne, P. Janis, C. Ribeiro, and K. Hugl "Device-to-Device Communications; Functional Prospects for LTE-Advanced Networks," in Proc. of IEEE International Conference on Communications Workshops, pp. 1-6, Jun. 2009. Article (CrossRef Link)

[3] C. H. Yu, O. Tirkkonen, K. Doppler, and C. Ribeiro, "On the Performance of Device-to-Device Underlay Communication with Simple Power Control," IEEE 69th Vehicular Technology Conference, 2009, pp. 1-5, Apr. 2009. Article (CrossRef Link)

[4] J. J. Lehtomäki, J. Vartiainen, M. Bennis, and K. Umebayashi, "Direct communication between terminals in infrastructure based networks," ICT-MobileSummit, pp. 1-5, Jun. 2008

[5] R. -S. Chang, Wei. -Y. Chen, and Y. -F. Wen, "Hybrid wireless network protocols," IEEE Transactions on Vehicular Technology, vol. 52, pp. 1099-1109, Jul. 2003. Article (CrossRef Link)

[6] T. Chen, G. Charbit, and S. Hakola, "Time Hopping for Device-To-Device Communication in LTE Cellular System," in Proc. of IEEE Wireless Communications and Networking Conference, Apr. 2010. Article (CrossRef Link)

[7] M. Zulhasnine, C. C. Huang, and A. Srinivasan, "Efficient resource allocation for device-to-device communication underlaying LTE network," in Proc. of IEEE 6th International Conference on Wireless and Mobile Computing, Networking and Communications, pp. 368-375, Oct. 2010. Article (CrossRef Link)

[8] T. K. Madsen, Q. Zhang, F. H. P. Fitzek, and M. Katz, "Design and Evaluation of IP Header Compression for Cellular-Controlled P2P Networks," IEEE International Conference on Communications, Jun. 2007, pp. 4046-4051. Article (CrossRef Link)

[9] T. Koskela, S. Hakola, T. Chen, and J. Lehtomaki, "Clustering Concept Using Device-To-Device Communication in Cellular System," in Proc. of IEEE Wireless Communications and Networking Conference, pp. 1-6, Apr. 2010. Article (CrossRef Link)

[10] K. Doppler, Klaus, C. H. Yu, C. B. Ribeiro, and P. Jänis, "Mode Selection for Device-To-Device Communication Underlaying an LTE-Advanced Network," in Proc. of IEEE Wireless Communications and Networking Conference, Apr. 2010. Article (CrossRef Link)

[11] [Online]. Available: http://www.trademarkia.com/flashlinq-77422968.htm

[12] C. H. Yu, O. Tirkkonen, K. Doppler, and C. Ribeiro, "Power Optimization of Device-to-Device Communication Underlaying Cellular Communication," in Proc. of IEEE International Conference on Communications, May, 2009, 1-5. Article (CrossRef Link)

[13] T. Peng, Q. X. Lu, H. M. Wang, S. Y. Xu, and W. B. Wang, "Interference Avoidance Mechanisms in the Hybrid Cellular and Device-to-Device Systems," IEEE 20th International Symposium on Personal, Indoor and Mobile Radio Communications, pp. 617-621, Sep. 2009. Article (CrossRef Link)

[14] M. Wellens, J. Wu, and P. Mahonen, Petri, "Evaluation of Spectrum Occupancy in Indoor and Outdoor Scenario in the Context of Cognitive Radio," in Proc. of 2nd International Conference on Cognitive Radio Oriented Wireless Networks and Communications, pp. 420-427, Aug. 2007. Article (CrossRef Link)

[15] P. Marques, J. Bastos and Atílio Gameiro, "Opportunistic use of $3 \mathrm{G}$ uplink licensed bands," in Proc. of IEEE International Conference on Communications, pp. 3588-3592, May. 2008. Article (CrossRef Link) 
[16] Q. X. Lu, T. Peng, H. M. Wang, S. Y. Xu, and W. B. Wang, "A Game-theoretic Approach to Distributed Power Control Algorithm for Hybrid Systems," IEEE 20th International Symposium on Personal, Indoor and Mobile Radio Communications, pp. 2822-2826, Sep. 2009. Article CrossRef Link)

[17] B. Kaufman and B. Aazhang, "Cellular Networks with an Overlaid Device to Device Network," in Proc. of 42nd Asilomar Conference on Signals, Systems and Computers, pp. 1537-1541, Oct. 2008. Article (CrossRef Link)

[18] S. Sesia, I. Toufik, and M. Baker, The UMTS Long Term Evolution : From Theory to Practice, Wiley, 2009, ch. 19.

[19] 3GPP TSG-RAN, "Physical layer aspects for evolved UTRA," 3GPP Technical Report, 3G TR 25.814, v7.1.0, Sep. 2006

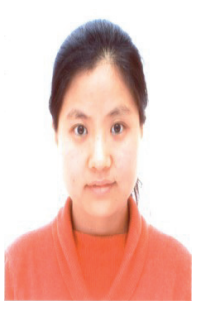

Shaoyi Xu received her Ph. D degree from Inha University, South Korea in 2007. After that, she worked in Nokia Research Center in Nokia China Ltd as a Senior Researcher and a Postdoctor. During that time, her research interests were mainly focusing on D2D communications and LTE standardization. From 2009 to now, as an associate professor, she worked in Research Institute of Broadband Wireless Mobile Communications, Beijing Jiaotong University. She has applied for 4 international patents and 10 Chinese patents, submitted 10 contributions to the Chinese or international standardization meetings and published more than 60 journal papers and conference papers. Her research interests include D2D communication, Cooperative communication in high-speed environments, Cognitive Radio and UWB, etc

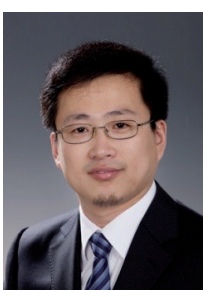

Haiming Wang received his Ph.D degree from Peking University at Beijing China in 2003. From 2003 to 2010, he served as a research specialist and research manager in Nokia, where he was responsible for the development and research of new wireless communication system for 3GPP standardization. From 2011, he was working in Renesas Mobile Europe (RME) as a Research manager. His focus area is mainly $3 \mathrm{GPP}$ relevant standard and LTE/LTE-A relevant research. He has published more than 40 technical papers and has more than 100 patent applications.

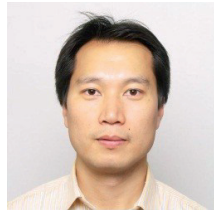

Tao Chen received his B.S. and M.S. degrees in Computer Science from Beijing University of Aeronautics and Astronautics, Beijing, P.R.China in 1998 and 2001, respectively. He is currently a principal researcher and 3GPP RAN4 delegate at Renesas Mobile Europe Ltd., Helsinki, Finland. Before joining Renesas Mobile Europe Ltd., he was a principal researcher and 3GPP RAN4 delegate at Nokia. His research interest includes standardization and research of next generation wireless communication systems. 


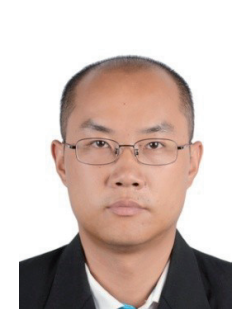

Tao Peng received his B.S. and M.S. degree from Beijing University of Posts an Telecommunications (BUPT) in 1999 and 2002 respectively. From 2002 to 2003 he worked in Nokia Research Center as a research engineer. Since 2003 he has worked in BUPT as a faculty, and received Ph.D. degree in 2010. Now he is an associate processor and his research interests focus on cognitive radio, hybrid cellular and Device-to-Device network, radio communication in white space spectrum, etc.

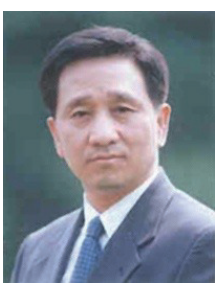

Kyung S. Kwak received the B.S. degree from the Inha University, Inchon, Kore in 1977, and the M.S. degree from the University of Southern California in 1981 and the $\mathrm{Ph} . \mathrm{D}$ degree from the University of California at San Diego in 1988, under the Inha University Fellowship and the Korea Electric Association Abroad Scholarship Grants, respectively. From 1988 to 1989 he was a Member of Scholarship Grants, respectively. From 1988 to 1989 he was a Member of
Technical Staff at Hughes Network Systems, San Diego, California. From 1989 to 1990 he was with the IBM Network Analysis Center at Research Triangle Park, North Carolina. Since then he has been with the School of Information and Communication, Inha University, Korea as a professor. He had been the chairman of the School of Electrical and Computer Engineering from 1999 to 2000 and the dean of the Graduate School of Information Technology and Telecommunications from 2001 to 2002 at the Inha University, Inchon, Korea. He is the current UWB Wireless Communications Research Center, a key government IT research center, Korea. He is now Inha Fellow Professor (IFP). Since 1994 he had been serving as a member of Board of Directors, and he had been the vice president for Korean Institute of Communication Sciences (KICS) during the term of 2002-2000 year. He was the KISC's president of 2006 year term. In 1993, he received Engineering College Young Investigator Achievement Award from Inha University, and a distinguished service medal from the Institute of Electronics Engineers of Korea distinguished service medal from the Institute of Electronics Engineers of Korea
(IEEK). In 1996 and 1999, he received distinguished service medals from the (IEEK). In 1996 and 1999, he received distinguished service medals from the
KICS. He received the Inha University Engineering Paper Award and the LG Paper Award in 1998, and Motorola Paper Award in 2000. His research interest include multiple access communication systems, mobile communication systems, UWB radio systems and ad-hoc networks, high-performance wireless Internet. Mr. Kwak is members of IEEE, IEICE, KICS and KIEE. 
IX

\title{
NETWORK CONTROLLED DEVICE-TO-DEVICE (D2D) AND CLUSTER MULTICAST CONCEPT FOR LTE AND LTE-A NET- WORKS
}

\author{
by \\ Seppälä, J., Koskela, T., Chen, T. and Hakola, S. 2011 \\ Proceedings of IEEE Wireless Communications and Networking Conference 2011
} (WCNC'11)

Reproduced with kind permission by IEEE 


\title{
Network Controlled Device-to-Device (D2D) and Cluster Multicast Concept for LTE and LTE-A Networks
}

\author{
Jaakko Seppälä*, Timo Koskela ${ }^{\dagger}$, Tao Chen ${ }^{\ddagger}$ and Sami Hakola ${ }^{\S}$ \\ ${ }^{*}$ Centre for Wireless Communications, University of Oulu, jaakko.markus.seppala@gmail.com \\ †t timo.koskela@ renesasmobile.com \\ $\ddagger$ tao.chen@ renesasmobile.com \\ §sami.hakola@ renesasmobile.com \\ TRenesas Mobile Corporation, Finland
}

\begin{abstract}
In this paper we introduce a reliable multicast concept for Device-to-Device (D2D) communication integrated into cellular network. In addition to the introduction of the basic concept, initial simulation results are presented as well. Clustering closely located devices which have local communication needs is a feasible and efficient way of solving the increasing data traffic requirements in the future cellular network. Reliable D2D multicast concept introduced in this paper is designed to be scalable and efficient solution for local communication needs uch as file transfer and even streaming services. Due to the such as rile the network involvent sufficient quality of service can be guaranteed. In addition, due to the flexible mode switching between direct and cellular modes in the integrated operation the service continuity can be provided.
\end{abstract}

\section{INTRODUCTION}

In a time span of just few years the growth of video and other data intensive traffic in the internet has been tremendous [1]. With the advent of $4 \mathrm{G}$ technologies and very capable handheld devices, it is certain that the amount of video traffic will see a manifold increase in cellular networks. The following research topics have gained interest to help alleviate the ever-growing need for bandwidth in cellular networks: Direct communication (device-to-device, D2D) and feature utilizing direct communication interface clustering of devices and wireless multicast transmission in the cluster.

In direct communication, terminals transmit data directly to each other without circumventing it through a base station (BS). Current standards enabling direct communication such as Bluetooth and Wireless LAN are often complicated to use and setup, and sometimes induce unnecessary steps at setting up the connection between terminals. They also operate on unlicensed bands and thus cannot guarantee Quality of Service (QoS). The concept of network controlled direct communication in a cellular network not only offers seamless operation and connection setup, but is also able to guarantee QoS since the band is licensed and resources are controlled by the network. Also, the regular cellular connection will always be available as a backup and ensure the service continuity in case the D2D link between devices deteriorates.

Compared to cellular communication, D2D both reduces interference to other users (reduced intercell interference), releases resources and increases the spatial reuse of radio resources, benefiting the network as a whole: The BS doesn't have to process the data D2D terminals are transmitting, thus allowing it to handle more connections. Moreover, direct communication has potential to offer smaller delays, thus better user experience, than its cellular counterpart, since the data doesn't have to traverse first to the BS and from there to the receiver. For the user the D2D setup and mode switching is transparent.

The other new technology enabled by direct communication, D2D multicast in the cluster, can offer substantial gains Imagine an information sharing scenario: Typically the user would have to send the same file separately to every receiver. With multicast, one transmission is enough - the same transmission goes to every receiver, saving both time and network resources. The multicast need not be limited to D2D mode. With modifications to the base station, multicast can be used in cellular mode also, providing service continuity to enhance the data sharing in cellular networks.

D2D range depends usually on the available transmission power and the interference environment. D2D communication mode is intended for local communication and thus the practical transmission range is some tens of meters. The key purpose is that it will complement the capabilities of the cellular network at specific sites: For example office blocks, campus areas, concert sites etc. in which potential users are located closely to each other, would be very suitable for applying D2D. At such venues the cellular network runs out of capacity occasionally. With D2D, the BS would be under a lot less strain and capable of housing more calls.

In this paper we concentrate on network controlled D2D and more particularily, multicasting. Network controls the resource allocation and transmission power of the D2D devices and is thus in full control of the interference caused by the D2D. On the other hand, network can also control the cellular to D2D interference. With proper mode selection the QoS and service continuity can be guaranteed for D2D users. Network controlled D2D also has a key advantage over uncoordinated 


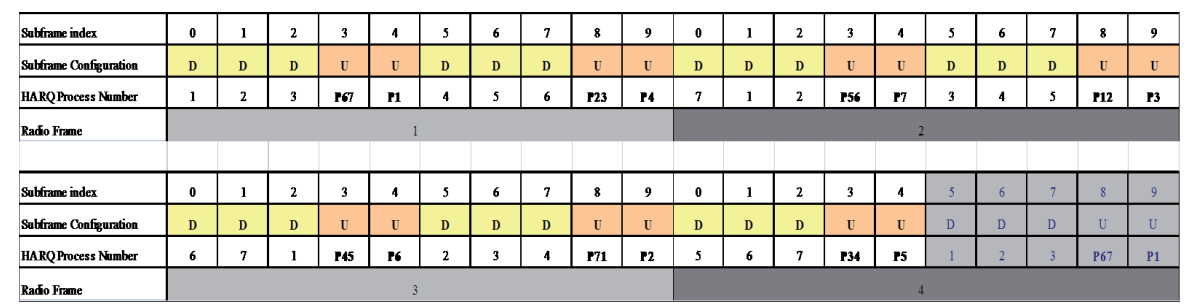

Fig. 1. Example TDD TX/RX configuration for multicasting (3DL/2UL).

device to device occuring e.g. in the unlicensed band: If the signal quality weakens during a communication session, the network may switch from D2D to cellular connection on the fly - the user need not concern with re-establishing a connection.

First we introduce the known challenges in reliable multicast and then provide some feature proposals for the multicasting concept to combat the challenges. Additionally, we provide initial results for the D2D multicasting concept with limited set of features implemented and compare it to the cellular multicasting.

\section{Cluster MulticAst CONCEPT}

A. Challenges in Reliable Multicast

Due to the nature of the wireless radio channel, link quality can be dramatically different for each receiver, even if they were located geographically close to each other. In unicast the problem would be mitigated by using a different transfer rate for each, depending on the channel quality. In multicast, transmitting to each receiver at different rates would add the system's complexity and in many cases be impractical. For example, were a streaming video sent to one receiver at a very high transfer rate, and at a lower rate to another, the first receiver would become subject to inacceptably long delays waiting for the second one to catch up. Thus it will become a challenging task to choose an optimal transfer rate for the multicast transmission.

The typical way of solving the problem is to use the worst link's transfer rate for every receiver. This way performance suffers for users with good link quality, but it is ensured that every user receives the data correctly. Because of this, it is important that the mode selection between D2D and cellular is done properly. The mode selection for the cluster communication is examined in more detail in [2]

In reliable multicast one of the key design challenges is the feedback. Feedback implosion is a known problem (short introduction e.g. in [3]) and unless it is considered in the design, it limits the scalability of the protocol.

\section{B. TDD duplexing on FDD Uplink}

The target system for this D2D concept study is LTE(-A) FDD (Frequency Division Duplexing). To minimize interfer- ence and optimize the use of radio resources, D2D uni- and multicast modes were implemented as TDD (Time Division Duplexing) communication, which operates in the FDD uplink channel. This way, downlink channel is left available for cellular communication.

LTE FDD uplink is divided to $10 \mathrm{~ms}$ radio frames. Each radio frame consists of two $5 \mathrm{~ms}$ half frames. One half frame is further divided in five $1 \mathrm{~ms}$ subframes and one subframe to two $0.5 \mathrm{~ms}$ slots [4]. Fig. 1 illustrates the example TX/RX slot configuration for the communication within the cluster. The 3 subframes downlink are configured for data transmission and 2 uplink subframes for ACK and NACK feedback messages. Feedback scheme presented in the figure is a synchronous hybrid automatic repeat request (HARQ) scheme, where HARQ process feedbacks are mapped to specific UL subframes. The whole feedback process requires 7 HARQ processes and it cycles through in 3.5 radioframes. Feedback delay for processes varies depending on which part of the cycle the certain process is feedbacked. Utilization of different TDD configurations offer possiblity for tradeoff between throughput and delay.

C. HARQ feedback (Common Feedback Region)

Located at medium access control (MAC) layer, HARQ is the lowest level of error correction in LTE.

In cluster multicast, HARQ level feedback is handled by joining every receiver's feedback messages into a common feedback region. The selected scheme is a NACK based feedback scheme where only an incorrectly received transmission is indicated back to the transmitter. If a receiver receives the packet correctly it sends no feedback. The multicast HARQ scheme is depicted in the figure 2. Based on the TDD configuration scheme in the previous section the two UL subframes are used for the feedback which enables that up to 4 HARQ processes can be mapped with the current scheme. Which HARQ processes are mapped at which time instant depends on the current subframe in the TDD TX/RX cycle.

D. RLC Feedback

In addition to the well-known HARQ mechanism at the physical layer, also RLC (Radio Link Control) level feedback 


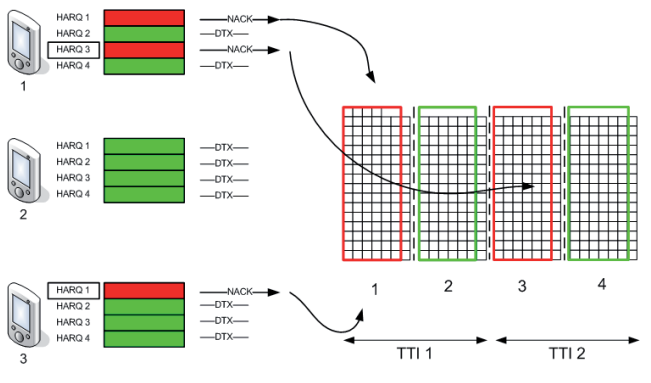

Fig. 2. Common Feedback Signaling, several HARQ processes are mapped to a common feedback resource.

is needed in order to cope with the residual BLER (Block Error Rate) after HARQ.

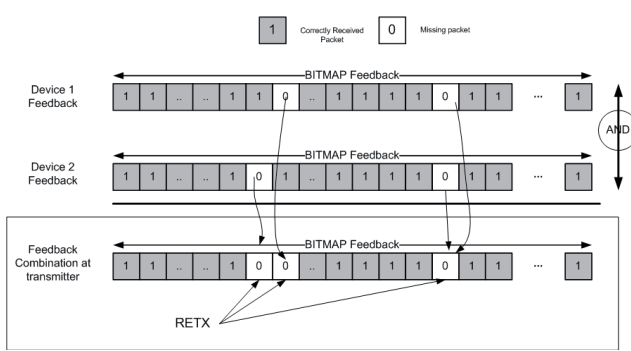

Fig. 3. Combination of RLC status reports at transmitting side.

RLC level retransmissions are based on combining the status reports from the receiving RLC entities. Transmitting side RLC entity is required to keep track of the correctly and incorrectly received RLC PDUs. The RLC feedback combination process is illustrated in figure 3. Transmitting RLC entity collects the feedback to bitmaps and performs an AND operation. After operation transmitter issues retransmissions for missing segments. Due to the combination with $A N D$ operation, certain receiving entities may receive duplicates of individual RLC packets. Duplicates are detected at RLC level and discarded.

Once all the packets have been received correctly by every receiver, the transmitter may move its RLC level transmission window to the next set of packets waiting in the buffer.

\section{E. Co-operative Features}

The NACK based feedback scheme also enables cooperative features to be implemented to the multicast concept. The co-operative retransmission scheme is illustrated in the figure 4 . In step 1 the cluster head $(\mathrm{CH})$ transmits data to session members. In Step 2 the members transmit feedback according to the scheme mentioned above. If a member has received the data correctly it does not respond anything to the cluster head and sets itself to feedback listening mode. Due to the synchronous operation of HARQ the other members can also listen to the HARQ feedback and retransmit the lost data together in the same HARQ process as illustrated in the step 3 . The member which did not receive the original transmission correctly can combine the multiple transmissions made by the cooperating members nearby and thus increase the probability for correct reception of the retransmission. The devices that do not hear each others feedback transmission will not participate in the co-operative retransmission.

However there are few challenges in this co-operative retransmission scheme:

- Feedback misinterpretation (decoding) errors. In case a NACK-based mutual signaling feedback scheme is used, the discontinuous transmission (DTX) is considered as an ACK and only NACK is transmitted. If even one of the nearby devices then decodes the DTX transmission to NACK it will retransmit and possibly interfere with the cluster head transmission of new data on that certain HARQ process.

- How to select the candidates for retransmission? Assuming that the original transmitter always retransmits it may not be useful that every member which has received data correctly participates in co-operative retransmission.
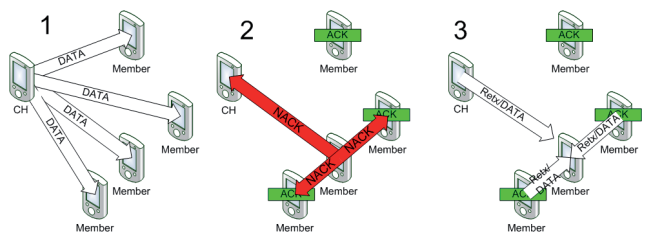

Fig. 4. Co-operative retransmission scheme.

\section{F. Cluster Management Based on Feedback}

By adding some intelligence into the handling of feedback messages, the cluster head can use them for managing the cluster members.

The NACKs from users can be soft combined with the common resource allocation as described in subsection II-C. The other option is to individually decode the NACKs with user specific sequence like cyclic shift CAZAC sequence and Walsh-Hadamard codes. Also, both techniques can be used in tandem.

For example, the NACKs for the first $\mathrm{N}$ transmissions would be soft combined since a lot of users may fail during the period. Then NACKs for the last M transmissions could use the user specific sequence to inform the transmitting device which user still failed.

With the help of the individually coded NACKs, transmitter will know exactly which receivers perform worst in a long 
term. This information could be used for intelligent cluster group management and in an efficient way.

\section{NS-2 IMPLEMENTATION}

A. General Description of the NS-2 Simulator

Network simulator 2 (NS-2) is a discrete event simulator targeted at networking research [5]. Programmed in C++ and OTcl languages, it is popular for its extensibility (due to the open source model) and of course, for being free of charge.

We have extended the NS-2 simulator to model accurately an LTE FDD system. In addition, D2D and direct multicast and cellular multicast features have been added to the model. Here the D2D communication operates on uplink band of the FDD cellular system.

The radio channel properties such as shadowing, penetration and path loss, doppler etc. are based on standards [6] and [7]. The link-to-system level interface is realized by employing exponential effective SINR mapping (EESM), which is explained in more detail in [8].

The network model supports user equipment (UE), BS, FBS (femto base station), TCP/IP router and core network nodes. The emphasis of this research is on data traffic, so the protocol stacks of the nodes are modeled accurately at user plane. The protocol stacks are inspected in more detail in subsection III-B. Control plane layers are also present in the nodes, but modeled in less detail.

Several traffic sources are available in the extended simulator: voice, data or streaming video sources. Voice sources are modeled as VoIP sources with AMR 12.2 codec. Data sources are HTTP and FTP sources, whereas streaming video sources are MPEG4 coded video sources. The traffic source models are based on [9], [10] and [11]

\section{B. Protocol Stack Implementation}

The user plane (data) protocol stacks for HTTP and FTP traffic are presented in figure 5. The protocol stacks for VoIP and streaming video differ from fig. 5 in that instead of TCP, they employ RTP/UDP over the IP layer.

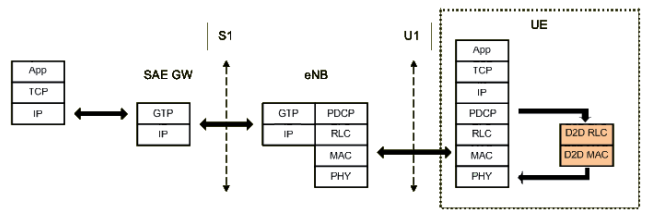

Fig. 5. LTE protocol stack with D2D layers.

In figure 5, SAE GW represents a network core entity. The eNB stands for enhanced node B, which is base station in LTE terminology. S1 and U1 are the interfaces between different nodes.

Cellular mode packets go through the normal LTE protocol stack, whereas D2D packets are diverted to a separate D2D
RLC/MAC stack by the PDCP layer. The D2D packets return to the normal LTE stack at PHY layer.

\section{C. eNB forwarding mode}

In cellular multicast the core network latencies are omitted by utilizing a so-called eNB forwarding mode. In this mode eNB is able to route internally the data, for which the source and destination cell is the same. In addition the eNB is able to multicast on downlink the data which is transmitted on uplink by a multicasting device.

D. Implementation Assumptions

In this study we assume several simplifications to the D2D and multicast implementations.

- Since the cluster management is not the main topic of this research, cluster members are pre-defined in the beginning of simulations and kept the same until the end of the simulation run.

- At HARQ level, common feedback is used in multicast, but the co-operative retransmission scheme is not utilized in the simulations. Only the original transmitter sends the retransmission and every device in the cluster receives it. The devices that receive a duplicate will drop the duplicate packet (Fig. 6).

- RLC level feedback is not present in multicast.

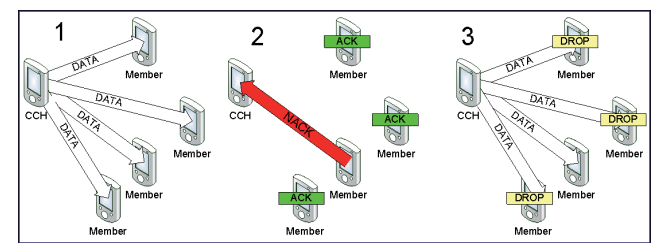

Fig. 6. Simplified retransmission scheme.

\section{Simulations}

A. Simulation Scenarios

The cell layout is one isolated cell with one sector. Only one multicast pair was placed in the cell and thus the interference and scheduling delays were omitted. Two cases were selected to study throughput performance and delay performance separately. Throughput performance (Scenario 1) was studied by using a full buffer traffic model, in which recording of delay and packet loss statistics were ignored. Delay performance (Scenario 2) was studied by generating low amount of traffic on application layer so that the buffering delay would not affect to the performance.

In these initial simulations, the HARQ with NACK based feedback scheme and RLC in UM mode were active. Utilization of co-operative features were omitted from these initial simulations. Also only one TDD configuration was used. 
TABLE I

SiMUlation PARAMETERS

\begin{tabular}{|c|c|}
\hline Parameter & Value \\
\hline Simulation drops & 100 \\
\hline Drop duration & $60 \mathrm{~s}$ \\
\hline Core Network Delay & $\begin{array}{l}\text { Ignored, eNB in BS forwarding } \\
\text { mode }\end{array}$ \\
\hline Cellular layout & Isolated cell, 1-sector \\
\hline Simulation Scenarios & $\begin{array}{l}\text { Scenario 1: full buffer Scenario 2: } \\
\text { light load }\end{array}$ \\
\hline Inter-site distance & $500 \mathrm{~m}$ \\
\hline Devices per sector & One multicast cluster \\
\hline Device start location & Uniformly randomly distributed \\
\hline Mobility & static scenarios \\
\hline D2D cluster sizes & $50 \mathrm{~m}, 25 \mathrm{~m}$ and $5 \mathrm{~m}$ \\
\hline Multicast cluster & 1 source UE, 3 target UEs \\
\hline $\begin{array}{l}\text { D2D TX/RX subframe configura- } \\
\text { tion }\end{array}$ & $3 \mathrm{DL} 2 \mathrm{UL}$ \\
\hline Distance dependent path loss & $\begin{array}{l}\text { Cell mode: } \\
128.1+37.6 \log _{10}(d[k m]) \\
\text { D2D mode, NLOS: } \\
40.0 \log _{10}(d[k m]) \\
30.0 \log _{10}(f)+49.0, \quad[\mathrm{f}] \quad \text { in } \\
\mathrm{Hz} \\
\begin{array}{ll}\text { D2D mode, LOS: } \\
16.9 \log _{10}(d[\mathrm{~m}]) \\
20.0 \log _{10}(f / 5.0)+ \\
{[\mathrm{f}] \text { in } \mathrm{GHz}}\end{array}+46.8, \\
\end{array}$ \\
\hline RLC Mode & UM \\
\hline Shadowing & Log-normal, similar to [12] \\
\hline Shadowing standard deviation & $8 \mathrm{~dB}$ \\
\hline $\begin{array}{l}\text { Antenna gains and patterns (trans- } \\
\text { mitter and receiver) }\end{array}$ & $\begin{array}{l}\text { BS: } 14 \mathrm{dBi} \text { Device: Omnidirec- } \\
\text { tional } 0 \mathrm{dBi}\end{array}$ \\
\hline Deployment center frequency & $2.0 \mathrm{GHz}$ \\
\hline Deployment Bandwidth & $5 \mathrm{MHz}$ \\
\hline Total BS tx power & $46 \mathrm{dBm}$ \\
\hline Cellular UE power class & $24 \mathrm{dBm}$ \\
\hline D2D Tx Power & 20dBm (fixed) \\
\hline Number of HARQ transmissions & 4 \\
\hline
\end{tabular}

We present four different multicast scenarios: The first is a cellular multicast case in which the transmitting device transmits to uplink and eNB then multicasts the transmission to receiveing cluster members. The other three are D2D multicast cases in which the receiving cluster members are placed at 50 $\mathrm{m}, 25 \mathrm{~m}$ and $5 \mathrm{~m}$ distance from the transmitting device. To rule out the scheduling delays at eNB in these simulations no other traffic is generated in the cell and the transmitting device was scheduled in every subframe.

The main simulation parameters are presented in table I.

B. Simulation Results

Simulation results for the Scenario 1 are presented in the figure 7. Results are plotted as CDF curves of the average throughputs observed in the different simulation drops. Different scenarios are named as " $5 \mathrm{~m} "$, , $25 \mathrm{~m} ", " 50 \mathrm{~m}$ " for D2D multicast and as "Cell" for the cellular multicast. Firstly it can be noted that the D2D throughputs at all distances have less variation than the cellular case. One reason for this is that the D2D performance is not sensitive to the cluster's location in the cell as the cellular multicast performance is very dependent on the transmitting device's distance from the eNB.

As it can be observed from the throughput figure the D2D multicast on $5 \mathrm{~m}$ distance has very solid performance, but in some cases the Cell outperforms the direct multicast. Basically this is due to the different duplexing machanisms. Cellular multicast operates in FDD mode and with good link quality toward the eNB the transmitting device is able to use highest available MCS (Modulation and Coding Scheme) and transmit constantly. D2D multicast operates on TDD mode the performance is mainly limited by the TDD subframe configuration. Here the maximum observed throughput for D2D is roughly $3 / 5$ of the maximum cellular throughput due the $3 \mathrm{DL} / 2 \mathrm{UL}$ subframe configuration. However, especially in the $5 \mathrm{~m}$ case the high SNR due to the short distance would enable the use of higher order modulations to further enchance the performance of the D2D multicast.

One other aspect that has to be considered when comparing the performance between cellular and D2D multicast cases. The results could be further scaled with spectral efficiency considerations. Cellular multicast requires resources from both uplink and downlink where as the D2D multicast only occupies the uplink resource. Also the feedback resources for the D2D multicast could be mapped to PUCCH (Physical Uplink Control Channel, a shared frequency/time resource reserved for User Equipment (UE) to transmit L1/L2 uplink control information) with negligible resource requirements for the HARQ feedback. This consideration would further improve spectral efficiency of D2D multicast.

Although the transmission power was fixed for D2D multicast in these simulations, the D2D could also enable power savings at the transmitting device especially with small distances.

Results for the Scenario 2, delay performance, are presented in the figure 8 . As in figure 7 the results are plotted as CDF curves of average values of each simulation drop. The delay results are recorded at the receiver side on top of the PDCP layer. Cellular multicast results include the delays of both uplink and downlink. As mentioned in the previous section the scheduling delays and core network delays were omitted from the delay calculations.

The delay performance retells the story that was observed from the throughput performance results. D2D multicast scheme has smaller variation in average delays and the overall delay performance is better than with cellular multicast, for the $5 \mathrm{~m}$ and $25 \mathrm{~m}$ the difference is significant. One thing affecting the cellular multicast delay is the BLER target and increased number of retransmissions due to the transmitter's uplink link quality. For D2D multicasting the selected TDD configuration plays also a role here: although the 3DL/2UL configuration may not be the best selection in terms of throughput for such highly asymmetric service as file sharing the delay performance is good. The utilization of TDD offers flexibility 
and possiblity for tradeoff between delay and throughput, based on the QoS requirements.

Based on the simulation results and observation following summary could be composed:

- The smaller variation on the throughput and delay in D2D multicasting cases would be useful to efficiently offer the advanced services such as the steaming services characterized by the constant bitrate (CBR) and delay sensitivity.

- Different from the performance of cell edge UEs in the cellular multicasting cases, D2D multicasting performance is not sensitive to the receving members' location in the cell. Instead, it is more sensitive to the proximity of cluster members.

- eNB controlled D2D multicasting is promising to improve the performance of the cell edge UEs, thereby complementing the cellular multicasting transmission.

- The delay performance is significantly improved. Throughput performance is superior when spectral efficiency is considered

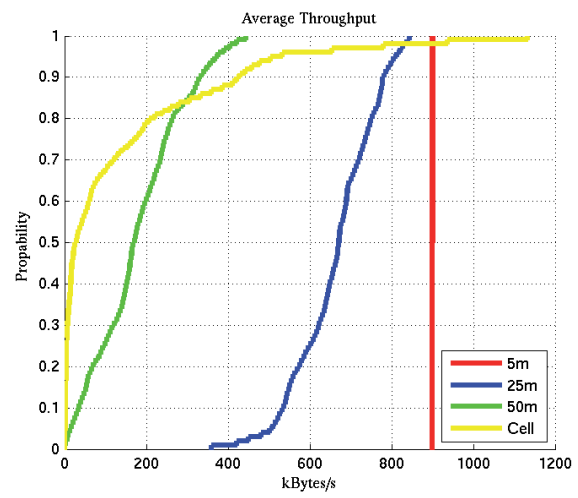

Fig. 7. Throughput values for D2D and cellular modes

\section{Conclusions}

In this paper we presented the set of features to support reliable D2D multicasting utilizing cellular resources. Also initial simulation results were provided to show that direct communication can enhance the throughput between closely located devices. All in all, D2D yields possibilities for great increases in throughput and also presents possibility for smaller delays compared to cellular multicast. As further work, the different features proposed in this paper could be implemented and the concept verified with additional simulations.

\section{REFERENCES}

[1] A. M. Odlyzko, "Internet traffic growth: Sources and implications," 2003.

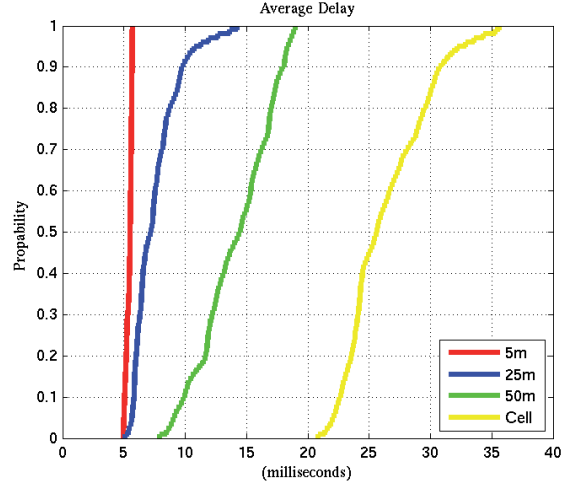

Fig. 8. Delay values for D2D and cellular modes.

[2] T. Koskela, S. Hakola, T. Chen, and J. Lehtomaki, "Clustering concept using device-to-device communication in cellular system," in WCNC 2010 Conference Proceedings. IEEE, April 2010.

[3] M. Ammar and D. Towsley, "Reliable multicast," http://www.cc.gatech.edu/ ammar/tutorial/html/qos/sld001.htm.

[4] 3GPP, "Ts 36.211 v9.1.0: Evolved universal terrestrial radio access (eutra); physical channels and modulation, technical report," 2010

[5] "Ns-2 simulator homepage," http://www.isi.edu/nsnam/ns.

6] 3GPP, "Tr 25.814 v. 7.1.0: Physical layer aspects for evolved universal terrestrial radio access (utra) (rel. 7), technical report," 2006.

[7] - "Tr 36.814 v. 1.5.0: Further advancements for e-utra physical layer aspects (rel 9), technical report" 2009

[8] A. K. A Tamimi, "Abstract exponential effective signal to noise ratio mapping (eesm) computation for wimax physical layer, masters thesis," Washington University in Saint Louis, MO, p. 70, May 2007

[9] V. Bolotin, Y. Levy, and D. Liu, "Characterizing data connection and messages by mixtures of distributions on logarithmic scale," in 16th Int. Teletraffic Congr., June 1999, pp. 887-894.

[10] B. Maglaris, D. Anastassiou, P. Sen, G. Karlsson, and J. Robbins, "Performance models of statistical multiplexing in packet video communications," IEEE Trans. Commun., vol. 36, no. 7, pp. 834-844, July 1988.

[11] M. Molina, P. Castelli, and G. Foddis, "Web traffic modeling exploiting tcp connections' temporal clustering through html-reduce," IEEE Network, vol. 12 , pp. 46-55, May-June 2000.

[12] 3GPP, "Tr 30.03u v. 3.2.0: Universal mobile telecommunications system (umts); selection procedures for the choice of radio transmission technologies of the umts, technical report," 1998. 